\title{
On the Scales of Turbulent Motion at High Reynolds Numbers
}

\author{
Dissertation \\ for the award of the degree "Doctor rerum naturalium" \\ of the Georg-August-Universität Göttingen \\ within the doctoral program \\ Physics of Biological and Complex Systems \\ of the Georg-August University School of Science \\ (GAUSS)
}

\author{
submitted by \\ Michael Sinhuber \\ from Oldenburg \\ Göttingen, 2015
}




\section{Thesis Committee:}

\section{Prof. Dr. Eberhard Bodenschatz}

Laboratory for Fluid Dynamics, Pattern Formation, and Biocomplexity, Max Planck Institute for Dynamics and Self-Organization

Prof. Dr. rer. nat. Dr.-Ing. habil Andreas Dillmann

Institut für Aerodynamik und Strömungstechnik,

Deutsches Zentrum für Luft- und Raumfahrt Göttingen

Prof. Dr. Marcus Müller

Institut für Theoretische Physik,

Georg-August-Universität Göttingen

\section{Dr. Gregory P. Bewley}

Laboratory for Fluid Dynamics, Pattern Formation, and Biocomplexity, Max Planck Institute for Dynamics and Self-Organization

\section{Members of the Examination Board:}

\section{Referee: Prof. Dr. Eberhard Bodenschatz}

Laboratory for Fluid Dynamics, Pattern Formation, and Biocomplexity, Max Planck Institute for Dynamics and Self-Organization

$2^{\text {nd }}$ Referee: Prof. Dr. rer. nat. Dr. habil. Andreas Dillmann

Institut für Aerodynamik und Strömungstechnik,

Deutsches Zentrum für Luft- und Raumfahrt Göttingen 


\title{
Further Members of the Examination Board:
}

\author{
Prof. Dr. Marcus Müller
}

Institut für Theoretische Physik,

Georg-August-Universität Göttingen

\section{Dr. Gregory P. Bewley}

Laboratory for Fluid Dynamics, Pattern Formation, and Biocomplexity, Max Planck Institute for Dynamics and Self-Organization

Prof. Dr. Jens Niemeyer

Institut für Astrophysik,

Georg-August-Universität Göttingen

\section{Prof. Dr. Gert Lube}

Institut für Numerische und Angewandte Mathematik,

Georg-August-Universität Göttingen

Date of oral examination: June $1^{\text {st }}, 2015$ 

"There is something fascinating about science. One gets such wholesale returns of conjecture out of such a trifling investment of fact."

Mark Twain, Life on the Mississippi, 1883 



\section{Contents}

$\begin{array}{ll}\text { Motivation } & 1\end{array}$

1 Introduction and Theory 3

1.1 The Equations of Motion . . . . . . . . . . . . . 3

1.2 A Statistical Approach to Turbulence . . . . . . . . . . . 7

1.3 Scaling and Intermittency $\ldots \ldots \ldots$

1.4 The Decay of Turbulence . . . . . . . . . . . . . . . 23

2 Experimental Methods 31

2.1 The Variable Density Turbulence Tunnel . . . . . . . . . . . . 32

2.2 The S1MA . . . . . . . . . . . . . . . 46

2.3 Hot-Wire Anemometry . . . . . . . . . . . . . . . . . . . . 49

2.4 Experimental Setup and Datasets . . . . . . . . . . . 56

3 Decay of Turbulence $\quad 71$

3.1 The Decay of Turbulent Kinetic Energy . . . . . . . . . . . 71

3.2 Turbulence-Intrinsic Description . . . . . . . . . . . . . . 88 
3.3 Effect of Grid Modifications . . . . . . . . . . . . . 101

3.4 Measurement Uncertainties . . . . . . . . . . . . . . . . . . 108

4 Scaling in Turbulence 115

4.1 Scaling in Inertial-Range Statistics . . . . . . . . . . . 115

4.2 The Third-Order Structure Function . . . . . . . . . . . . . 120

4.3 Extended Self-Similarity . . . . . . . . . . . . 126

4.4 Effective Scaling Exponents . . . . . . . . . . . . . . . . . . 134

4.5 Measurement Uncertainties . . . . . . . . . . . . . . . . . . . 141

5 Statistics of the Small Scales 147

5.1 Flow Properties . . . . . . . . . . . . . . . . . . . 147

5.2 Statistics of the Velocity Derivatives . . . . . . . . . . 153

5.3 The Build-Up of non-Gaussianity . . . . . . . . . . . . . 160

6 Discussion and Outlook 165

$\begin{array}{lr}\text { Appendix } & 169\end{array}$

A Experimental Conditions . . . . . . . . . . . . . . . 169

B Scaling Exponents Comparison . . . . . . . . . . . . 172

$\begin{array}{ll}\text { Bibliography } & 175\end{array}$

$\begin{array}{ll}\text { Curriculum Vitae } & 195\end{array}$

$\begin{array}{lr}\text { Acknowledgments } & 199\end{array}$ 


\section{Motivation}

Turbulence is a physical state of a fluid far from equilibrium. In turbulent flows, a huge number of degrees of freedom is excited and a wide range of interacting scales determines the flow characteristics. Turbulent flows are nonlinear and non-local. They exhibit chaotic spatial and temporal dynamics and extreme events are likely to occur. The air we breath is turbulent, the tea we drink is turbulent and the wind we feel is turbulent. Turbulent flows play an important role for earth's magnetic field (Batchelor 1950), for the climate (Bodenschatz et al. 2010) as well as for the formation of stars (Krummholz \& McKee 2005). Knowledge of turbulence is needed to build bridges that do not collapse (Lin \& Ariaratnam 1980) and to build planes that fly (Kuchemann 1965).

Scientific research on turbulence started many centuries ago, with Leonardo da Vinci drawing detailed pictures of the characteristics of a turbulent flow (see, e.g., Argyris et al. (2010, p. 618)). The equations of motion still used today to describe turbulent flows were derived by Navier (1827) and Stokes (1845). Over the centuries, many renowned and excellent physicists and mathematicians investigated turbulence in great detail. However, up to today, there is no unified theory of turbulence, very 
few exact predictions from the governing equations are available and the precise predictability of the behavior of turbulent flows is limited.

For example, we can not precisely predict how long it takes for a turbulent flow in a stirred coffee cup to come to rest once the stirring has stopped. We know that the turbulent kinetic energy in this decaying flow gets eventually dissipated into heat. Yet, we do not know the exact statistics of key flow parameters like the velocity field during the decay process. We also do not precisely know the statistics of the physical process dissipating turbulent kinetic energy into heat. Additionally, we do not know exactly, how these quantities depend on the turbulent flow's vigorousness that is given by the so-called Reynolds number.

Chapter 1 introduces the equations of motion of turbulent flows, as well as the fundamental theoretical frameworks to describe the statistical properties of turbulence. In chapter 2, the experimental setup and measurement techniques are explained. The large-scale based decay of turbulence and its dependence on the Reynolds number is investigated in chapter 3 . In chapter 4, the scaling properties of turbulence at the intermediate scales are discussed. Chapter 5 addresses the small-scale statistics of turbulence. The results are summarized in chapter 6 , in which an outlook to future research possibilities on questions beyond the scope of this thesis is given as well.

Parts of this thesis have been published in Review of Scientific Instruments (Bodenschatz, Bewley, Nobach, Sinhuber \& Xu 2014) and Physical Review Letters (Sinhuber, Bodenschatz \& Bewley 2015). 


\section{Introduction and Theory}

In this chapter, I present the theoretical background of turbulence research based upon the detailed descriptions in the widely known textbooks by Argyris et al. (2010), Davidson (2004), Frisch (1995), Monin \& Yaglom (2007) and Pope (2000), as well as from the original publications wherever appropriate. The aim of this chapter is to focus on the concepts and frameworks that predict the behavior of statistical quantities at different length-scales of a turbulent flow. Section 1.1 introduces the governing equations of a turbulently moving fluid, whereas section 1.2 presents a statistical approach to turbulence, including the famous theory of Kolmogorov (1941b) and its rich predictions. Section 1.3 explains the concepts of self-similarity and briefly derives the properties of velocity increment statistics. In section 1.4, the most prominent predictions on the decay of turbulence are reviewed.

\subsection{The Equations of Motion}

Let us consider an everyday fluid of finite volume in a cylindrical container: a glass of water. The classical approach to this physical problem in the spirit of Newton 
would be to describe the motion of the fluid by writing and solving the equations of motion of the individual water molecules, obtaining complete knowledge about the dynamics of the system. Assuming that a typical glass contains $300 \mathrm{ml}$ of water, this translates into the trajectories of $\mathcal{O}\left(10^{25}\right)$ water molecules along with their respective initial conditions and interactions. One can easily see that even by completely neglecting ions, additives and interactions with the atmosphere, solving this problem is not feasible. However, as the smallest scales produced by stirring the water would be of the order of $10^{-4} \mathrm{~m}$ (Wang et al. 2014) and the interaction distance between the water molecules themselves are several orders of magnitude smaller, in the nanometer range (Mortimer 2001), one can treat the fluid space as continuous and the discrete interactions between the individual molecules do not matter. This is the so-called continuum approximation, which allows for a field description of fluid motion. The same holds true for gases, as long as the mean free path of the molecules is much shorter than the smallest scales of the flow geometry. This is true for virtually all gases under standard conditions.

\subsubsection{The Navier-Stokes Equations}

The equations of motion for a fluid can be derived from the basic conservation laws, the conservation of mass and the conservation of momentum. Consider a continuous fluid with a density distribution $\rho(\mathbf{x}, t)$ and pressure $p(\mathbf{x}, t)$. Here, $\mathbf{x}$ denotes a position in space and $t$ the time. The motion is described by the velocity field $\mathbf{u}(\mathbf{x}, t)$. Conservation of mass can be expressed in terms of the continuity equation (Argyris et al. 2010, p. 463), 


$$
\frac{\partial \rho}{\partial t}+\nabla \cdot(\rho \mathbf{u})=0
$$

At low velocities, $u$, compared to the speed of sound, $c$, and thus low Mach numbers, $u / c$, most liquids and gases can be considered to be incompressible and of constant density in space and time. This holds true as long as the dimensions of the flow are small enough for gravitational density variations to be neglected. With this, equation (1.1) simplifies to the incompressibility condition

$$
\nabla \cdot \mathbf{u}=0
$$

Unless stated otherwise, a constant density $\rho(\mathbf{x}, t)=\rho$ is assumed throughout this thesis and all fluids are assumed to satisfy equation (1.2). Obeying Newton's Second Law, the total momentum of an element of an incompressible fluid can only change due to surface forces and volume forces acting on it, such that

$$
\rho\left(\frac{\partial}{\partial t}+\mathbf{u}(\mathbf{x}, \mathbf{t}) \cdot \nabla\right) \mathbf{u}(\mathbf{x}, t)=\rho \mathbf{f}(\mathbf{x}, t)+\nabla \cdot \sigma(\mathbf{x}, t)
$$

Here, $\mathbf{f}(\mathbf{x}, t)$ denote the volume force acting on the fluid element due to, e.g., gravity. The stress tensor $\sigma(\mathbf{x}, t)$ contains all information about the surface forces on the given fluid element due to pressure and molecular friction. For an incompressible 
fluid with constant dynamic viscosity $\mu$, the stress tensor is given by

$$
\nabla \cdot \sigma(\mathbf{x}, t)=-\nabla p(\mathbf{x}, t)+\mu \Delta \mathbf{u}(\mathbf{x}, t)
$$

Inserting equation (1.4) into equation (1.3), using the incompressibility condition in equation (1.2) and dividing by $\rho$ yields the Navier-Stokes equations,

$$
\left(\frac{\partial}{\partial t}+\mathbf{u}(\mathbf{x}, \mathbf{t}) \cdot \nabla\right) \mathbf{u}(\mathbf{x}, t)=-\frac{1}{\rho} \nabla p(\mathbf{x}, t)+v \Delta \mathbf{u}(\mathbf{x}, t)+\mathbf{f}(\mathbf{x}, t)
$$

where $v=\mu / \rho$ is the kinematic viscosity. For convenience, it is useful to introduce dimensionless quantities based upon the characteristic scales of the turbulent motion. With the characteristic length $L$, characteristic time $T$ and velocity $U=L / T$, one can rescale equation (1.5) by replacing $\mathbf{u}, t, \mathbf{x}, p, f$ and the differential operators by their dimensionless counterparts to obtain the Navier-Stokes equations in their well-known dimensionless form (Navier 1827, Stokes 1845):

$$
\left(\frac{\partial}{\partial t}+\mathbf{u}(\mathbf{x}, \mathbf{t}) \cdot \nabla\right) \mathbf{u}(\mathbf{x}, t)=-\nabla p(\mathbf{x}, t)+\frac{1}{\operatorname{Re}} \Delta \mathbf{u}(\mathbf{x}, t)+\mathbf{f}(\mathbf{x}, t)
$$

The dimensionless Reynolds number $\operatorname{Re}=\frac{U L}{v}$ (Reynolds 1883) is a measure of the ratio of inertial to viscous forces and gives information about how vigorous the turbulence is. 
Together with a full set of initial and boundary conditions, the Navier-Stokes equations are a set of nonlinear partial differential equations that describe the motion of a fluid. Furthermore, the equations are non-local due to the pressure gradient, a quantity that couples to the velocity field over an infinitely extended space. This can be seen by computing the divergence of the Navier-Stokes equations, yielding a Poisson equation for the pressure, which can be solved in terms of Green's functions (Argyris et al. 2010, p. 624):

$$
p(\mathbf{x}, t)=\int \frac{1}{4 \pi\left|\mathbf{x}-\mathbf{x}^{\prime}\right|} \sum_{i, j} \frac{\partial u_{i}\left(\mathbf{x}^{\prime}, t\right)}{\partial x_{j}} \frac{\partial u_{j}\left(\mathbf{x}^{\prime}, t\right)}{\partial x_{i}} \mathrm{~d} \mathbf{x}^{\prime}
$$

Obtaining the pressure at a single point in space demands the knowledge of the velocity field at every point in space, resulting in the non-locality of the NavierStokes equations.

\subsection{A Statistical Approach to Turbulence}

The combination of nonlinearity and non-locality makes the Navier-Stokes equations notoriously difficult to tackle and very few exact results and predictions are available. The equations are purely deterministic, yet, due to the nonlinearity and the involved degrees of freedom, they exhibit a strong dependence on minute variations in the initial conditions, thus leading to deterministic chaos. As a matter of fact, as of today there is not even a strict mathematical proof for the existence of smooth solutions for the Navier-Stokes equation given arbitrary initial and boundary conditions of 
sufficient regularity. This problem is deemed to be so important that it is on the list of Millennium Problems of the Clay Mathematics Institute ${ }^{1}$. Despite the open mathematical question as to whether the Navier-Stokes equations are meaningful descriptions of nature, there is no hint that they are not an adequate tool to precisely describe the motion of a real fluid within the limitations given above. As mentioned, small variations in initial conditions have drastic effects on the outcome of an individual realization of an experiment due to the deterministic, chaotic behavior of turbulent flows. However, statistical quantities as, e.g., averages or probability density functions are remarkably reproducible in turbulent flows and have been proven to be useful tools in understanding the underlying processes (Argyris et al. 2010, p. 654). Treating a turbulent flow similar to a random field, one can introduce statistical ensemble averaging to obtain meaningful averages. Let $\mathbf{x}_{n}$ be a random variable that can be measured in an experiment. The ensemble average $\langle\mathbf{x}\rangle$ can the be calculated by independent repetitions of the experiment as

$$
\langle\mathbf{x}\rangle=\lim _{N \rightarrow \infty} \frac{1}{N} \sum_{n=1}^{N} \mathbf{x}_{n} .
$$

Since independent repetitions of a single experiment are usually not realistically feasible, the averaging process in equation (1.8) is often replaced with a time average over the measurement time $T$ for the measurement variable $\mathbf{x}(t)$ via

\footnotetext{
${ }^{1}$ http://www.claymath.org/millenium-problems /

navier-stokes-equation (as of 23.1.2015, 15:20)
} 


$$
\langle\mathbf{x}(t)\rangle=\lim _{T \rightarrow \infty} \frac{1}{T} \int_{0}^{T} \mathbf{x}\left(t+t^{\prime}\right) \mathrm{d} t^{\prime}
$$

Obviously, measurement times are not infinite, so for equation (1.9) to be valid, the limit must converge even for finite $T$. If this is the case, then the turbulent flow in question is stationary.

\subsubsection{The Kolmogorov 1941 Framework}

Since exact predictions from the Navier-Stokes equations are rare, turbulence research must rely on thoughtful hypotheses and careful modeling. One of the most prominent concepts for the structure of turbulence dates back to Richardson (1922). Analyzing atmospheric data using Fourier methods, he envisioned turbulence consisting of a multitude of overlapping eddies, flow structures with characteristic length scales carrying a certain amount of kinetic energy. In his description, energy that is injected at a large scale $L$ of a three-dimensional system produces eddies of that size which carry the kinetic energy. These high Reynolds number structures will turn unstable, break up and create more eddies of smaller size, which will then carry the kinetic energy. These eddies will become unstable as well, producing even smaller eddies. This process continues with an energy transfer rate $\varepsilon$ until the size of the eddies becomes so small that viscous dissipation becomes important and dissipates the kinetic energy into heat at a length scale $\eta$. This concept is known as the energy cascade. It is the basis of the famous turbulence theory by Kolmogorov 
(1941b) and is still widely accepted in its core predictions. Kolmogorov (1941b) refined Richardson's cascade model based upon three hypotheses. His first hypothesis is based upon the observation that during the cascade process, turbulent structures seem to lose information about their genesis and obtain special symmetries. This is formulated in Kolmogorov's hypothesis of local isotropy, which can be restated in the following way (Pope 2000, see p. 184):

\section{Given sufficiently high Reynolds numbers, the motion of the small scales in turbulence is statistically homogeneous and isotropic.}

This must be understood as follows. Let $\mathbf{A}(\mathbf{x}, t)$ be an arbitrary quantity and $\mathbf{x}$ and $\mathbf{x}^{\prime}$ be positions in space. This quantity is called homogeneous if its ensemble average does not depend on $\mathbf{x}$ and thus fulfills: $\langle\mathbf{A}(\mathbf{x}, t)\rangle=\left\langle\mathbf{A}\left(\mathbf{x}^{\prime}, t\right)\right\rangle$. A two-point quantity $\mathbf{B}\left(\mathbf{x}, \mathbf{x}^{\prime}, t\right)$ is called isotropic if its ensemble average does not depend on the direction of the vector $\mathbf{x}-\mathbf{x}^{\prime}$ and therefore follows: $\left\langle\mathbf{B}\left(\mathbf{x}-\mathbf{x}^{\prime}, t\right)\right\rangle=\left\langle\mathbf{C}\left(\left|\mathbf{x}-\mathbf{x}^{\prime}\right|, t\right)\right\rangle$

The second and third hypotheses of Kolmogorov's theory concern the universality of turbulent flows and the flow parameters that characterize them. His first similarity hypothesis formulates the disconnection between the turbulent flow at small scales and the large scales $L$ (following Pope (2000, p. 185)):

\section{In a turbulent flow of large Reynolds numbers, the statistics of the small scales $r<<L$ have a universal form only dependent on the kinematic viscosity $v$ and the energy dissipation rate $\varepsilon$.}

With this hypothesis and the use of dimensional analysis, one is able to define length, time, and velocity scales of the small structures of the turbulent motion, the 
so-called Kolmogorov microscales. These scales are the size $(\eta)$, the characteristic velocity $\left(u_{\eta}\right)$ and the turn-over time $\left(\tau_{\eta}\right)$ of the smallest eddies in the turbulent flow (Pope 2000, p. 128),

$$
\begin{aligned}
\eta & =\left(v^{3} / \varepsilon\right)^{1 / 4} \\
u_{\eta} & =(\varepsilon v)^{1 / 4} \\
\tau_{\eta} & =(v / \varepsilon)^{1 / 2}
\end{aligned}
$$

With these quantities one is able to construct the Reynolds number defined over

the smallest scales $\operatorname{Re}_{\eta}=\frac{u_{\eta} \eta}{v}=1$. Empirically, one finds that the separation of the small and the large scales increases with Reynolds number as $L / \eta \sim \operatorname{Re}^{3 / 4}$. For very large Reynolds numbers, this led Kolmogorov to the hypothesis that there exists a range of scales which is neither affected by the large nor by the small scales, the so-called inertial range. It is formulated in Kolmogorov's second similarity hypothesis (following Pope (2000, p. 186)):

At very high Reynolds numbers, there exists a range of scale $\eta<<$ $r<<L$ at which the statistics of the turbulent motion have a universal form which does uniquely depend on $\varepsilon$ and not on $v$.

In this framework by Kolmogorov (K41), high-Reynolds number turbulence is pictured as the energy cascade which is fed by energy injection at large scales $L$. The cascade transfers energy with an energy dissipation rate $\varepsilon$ towards smaller scales. At the intermediate scales, the statistics of the K41 turbulence are solely 
determined by $\varepsilon$, whereas at the small scales dissipation starts to affect the statistics (see figure 1.1).

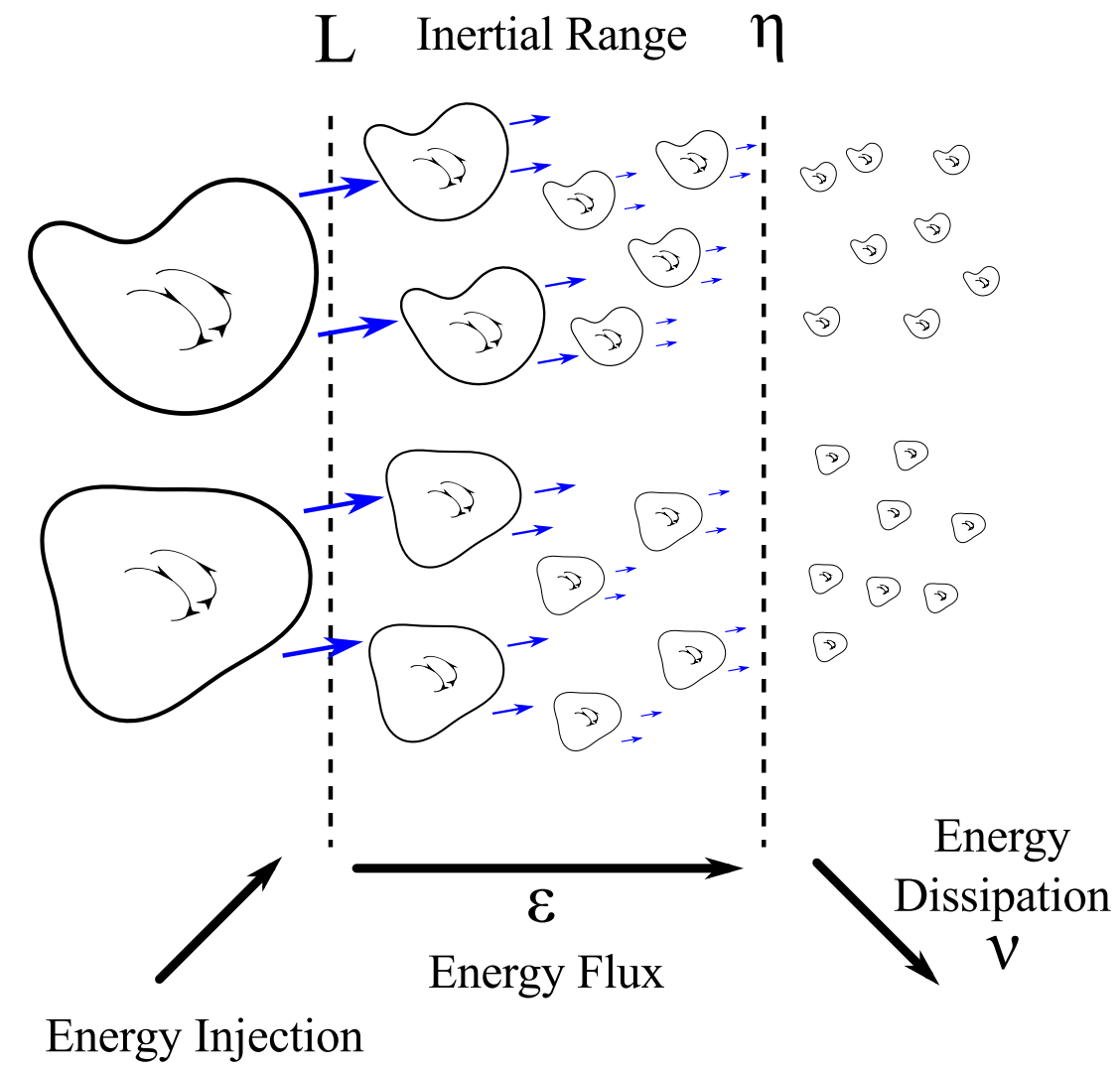

Figure 1.1: A sketch of the energy cascade in the K41 picture following Frisch (1995).

The K41 framework, despite its simplicity, is a powerful tool in predicting statistical behavior of turbulent flows. For example, one can get the shape of the energy spectrum $E(k)$ in the inertial range with simple dimensional arguments. Let $r$ be a length scale and $k=2 \pi / r$ its corresponding wavenumber. The kinetic energy $E_{a b}$ between two wavenumbers $k_{a}$ and $k_{b}$ is then defined as 


$$
E_{a b}=\int_{k_{a}}^{k_{b}} E(k) \mathrm{d} k
$$

From Kolmogorov's second hypothesis, it follows that in the inertial range, $E(k)$ is solely a function of the energy dissipation rate $\varepsilon$ and the wavenumber $k$ itself. As the energy spectrum has the dimensions $\mathrm{m}^{3} / \mathrm{s}^{2},[\varepsilon]=\mathrm{m}^{2} / \mathrm{s}^{3}$ and $[k]=1 / \mathrm{m}$, there is only one functional form for $E(k)$ which follows Kolmogorov's second similarity hypothesis:

$$
E(k) \sim \varepsilon^{2 / 3} k^{-5 / 3}
$$

Following K41, the energy spectrum should have a clear power-law behavior in the inertial range.

\subsubsection{The Integral Length Scale}

Thus far, the scale $L$ was assumed to be the scale at which energy is injected into the system. In a stirred glass of water, e.g., the energy injection scale would be equivalent to the scale of the stirring. As this scale is neither well defined nor measurable (e.g. in atmospheric turbulence) the definition of an equivalent but computable quantity is needed. This is possible from the statistics of the turbulent flows itself. The size of the largest eddies in a turbulent flow, the largest 
distances over which velocity fluctuations are spatially correlated, are connected to the energy injection scale. In a homogeneous, isotropic turbulent flow, one can use the one-component, longitudinal autocorrelation function $C(r)=\langle u(\mathbf{x}+\mathbf{r}, t) u(\mathbf{x}, t)\rangle$ to define the largest scales. Here $u$ is the velocity component along the separation vector $\mathbf{r} . C(r)$ is a measure for the correlation between the velocity component at the positions $\mathbf{x}$ and $\mathbf{x}+\mathbf{r}$. As the turbulent flow is assumed to be isotropic, $C(r)$ can only depend on the scalar separation $r=|\mathbf{r}|$, which leads to $C(r)=R(r) C(0)$. The large scale in the turbulent flow, the integral length scale, is then defined by means of the integral over the correlation function. Empirically, one finds that the correlation decays exponentially, making it possible to define the integral length scale as (Argyris et al. 2010, p. 660)

$$
L=\int_{0}^{\infty} \frac{C(r)}{C(0)} \mathrm{d} r
$$

One can easily see that it is not possible to measure infinite separations, but as the autocorrelation function decays quickly, it is possible to estimate the influence of large separations and the above definition remains useful.

\subsubsection{The Taylor Length Scale}

As dissipation already affects turbulent flows at scales somewhat larger than the Kolmogorov scale $\eta$, it is useful to define a length scale at which dissipation effects vanish. In an effort to systematically define such a length scale, Taylor (1935) 
constructed a length scale from the velocity autocorrelation function intended to give an estimate for the extent of the influence of dissipative effects. He defined the Taylor length scale $\lambda$ as the intersection between a parabolic fit to the peak of the autocorrelation function and 0 . Though this artificial quantity has no precise physical meaning, it serves as a useful tool for estimating the lower bound of the inertial range due to dissipative effects. One can show that the Taylor length scale can be computed from velocity derivatives of one velocity component $u$ along one direction $x$ as (Frisch 1995, p. 61)

$$
\frac{u^{\prime 2}}{\lambda^{2}}=\left\langle\left(\frac{\partial u}{\partial x}\right)^{2}\right\rangle
$$

with $u^{\prime}$ being the root mean square of this velocity component. The most common use of the Taylor length scale is to define a Reynolds number that only depends on flow characteristics, the Taylor Reynolds number, given by

$$
\mathrm{R}_{\lambda}=\frac{u^{\prime} \lambda}{v}
$$

For isotropic, homogeneous turbulence, this Reynolds number is unambiguously determined by the physics of the flow itself. Compared to the Reynolds number based on the larges scales $\operatorname{Re}_{\mathrm{L}}$, the Taylor Reynolds number scales as

$$
\mathrm{R}_{\lambda} \propto \sqrt{\mathrm{Re}}
$$




\subsubsection{Taylor's Frozen Flow Hypothesis}

Many of the predictions for turbulent flows concern the statistical behavior of velocity increments $\delta \mathbf{u}$, defined as

$$
\delta \mathbf{u}(\mathbf{x}, \mathbf{r}, t)=\mathbf{u}(\mathbf{x}+\mathbf{r}, t)-\mathbf{u}(\mathbf{x}, t),
$$

for a spatial separation vector $\mathbf{r}$. However, many experimental setups are only able to measure velocity increments in time, defined as

$$
\delta \mathbf{u}(\mathbf{x}, t, \tau)=\mathbf{u}(\mathbf{x}, t+\tau)-\mathbf{u}(\mathbf{x}, t)
$$

A measurement of the above quantity can for example be realized by one stationary measurement probe measuring at a single position for long times. A priori, it is unclear whether these two quantities share any statistical similarities. According to Taylor (1938), it is possible to translate spatial and temporal measurements given some specific flow conditions. For a flow with a velocity field $\mathbf{u}(\mathbf{x}, t)$ which consists of a strong mean flow $\mathbf{U}=\langle\mathbf{u}(\mathbf{x}, t)\rangle$ and small velocity fluctuations $\mathbf{u}^{\prime}(\mathbf{x}, t)$, such that $\mathbf{u}(\mathbf{x}, t)=\mathbf{U}+\mathbf{u}^{\prime}(\mathbf{x}, t)$, then, one can translate spatial separations $\mathbf{r}$ into temporal separations $\Delta t$ via 


$$
\mathbf{r}=\mathbf{U} \Delta t, \text { for } \quad|\mathbf{U}|^{2}>>\left\langle\left|\mathbf{u}^{\prime}\right|^{2}\right\rangle
$$

The basic consideration here is (see figure 1.2) that if a patch of turbulence is swept over a measurement device, as long as the mean speed of this sweeping is much larger than the turbulent velocities, by the time the patch has fully passed the measurement device, its internal flow structure has not changed at all. A measurement in time can thus be translated into a spatial measurement (Monin \& Yaglom 2007, p. 363).

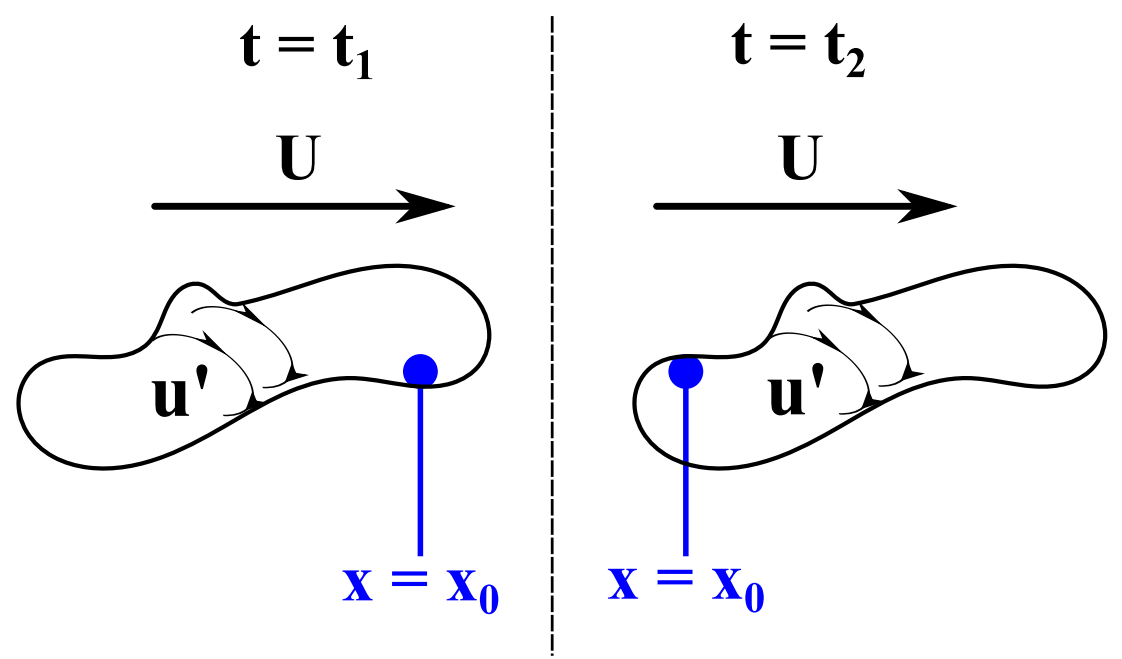

Figure 1.2: A sketch of Taylor's Frozen Flow Hypothesis. A patch of turbulence is swept over a measurement device (depicted in blue) at position $\mathbf{x}_{0}$ with a mean speed $\mathbf{U}$. If the mean speed $\mathbf{U}$ is much larger than the fluctuating velocity $\mathbf{u}^{\prime}$, then the patch does not change significantly while being swept over the measurement device. 


\subsection{Scaling and Intermittency}

Among the few exact results derived from the Navier-Stokes equations, one concerns the behavior of the longitudinal structure functions (Frisch 1995, p. 139):

$$
S_{n}(r)=\left\langle\delta u^{n}\right\rangle:=\left\langle\left((\mathbf{u}(\mathbf{x}+\mathbf{r}, t)-\mathbf{u}(\mathbf{x}, t)) \cdot \frac{\mathbf{r}}{|\mathbf{r}|}\right)^{n}\right\rangle
$$

These are the moments of the velocity increment component along the longitudinal direction. For homogeneous, isotropic turbulence, $S_{n}(r)$ can only depend on the absolute value of the separation vector $r=|\mathbf{r}|$. One can relate the longitudinal structure function to the probability density function of the longitudinal velocity increments $f(\delta u, r)$ with

$$
S_{n}(r)=\int \delta u^{n} \cdot f(\delta u, r) \mathrm{d} \delta u
$$

The probability density function contains all information about the statistics of the velocity increments, whereas the structure functions are connected to the statistics of increments within a certain band of magnitudes, while the statistics of increasing order $n$ are increasingly biased towards the extreme events.

\subsubsection{Kolmogorov's Four-Fifths Law}

Kolmogorov (1941a) reformulated an exact equation derived by de Kármán \& 
Howarth (1938) from the energy balance of the Navier-Stokes equations (1.5) in terms of the longitudinal structure functions under the assumptions of stationary, homogeneous, isotropic turbulence,

$$
S_{3}(r)-6 v \frac{\mathrm{d}}{\mathrm{d} r} S_{2}(r)=-\frac{4}{5}\langle\varepsilon\rangle r+q(r)
$$

Here, $q(r)$ is a source term containing the information about the energy injection at scale $r$. Within the limit of negligible viscosity, $v \rightarrow 0$, the second term on the left hand side of the equation vanishes as long as the derivative remains finite. Additionally, in the inertial range, there is no energy injection into the system, therefore $q(r)$ is zero as well. One thereby obtains Kolmogorov's famous four-fifths law for the behavior of the third-order structure function in the inertial range,

$$
S_{3}(r)=-\frac{4}{5}\langle\varepsilon\rangle r
$$

This result predicts a remarkably simple form of the third-order structure function considering the deterministically chaotic nature of turbulent flows. Referring back to the introduction of section 1.2, although the instantaneous velocity field eludes any concrete prediction, statistical measures like the third-order structure function are surprisingly robust quantities that follow relatively simple laws. 


\subsubsection{Self-Similarity in Turbulence}

One early observation in the study of turbulent flows was their apparent selfsimilarity. Figuratively speaking, a turbulent flow observed at a certain scale shows the same features as the same flow observed at a much larger or much smaller scale. This can for example by seen in high-resolution pictures of large atmospheric clouds, the shapes observed at the very small scales resemble the ones at the larger scales. Using the not-so-far-fetched assumption that turbulent flows are indeed self-similar, one can generalize Kolmogorov's four-fifths law (1.25) for arbitrary orders $n$. Following the elegant description in Argyris et al. (2010), one needs to define self-similarity in a mathematically correct way (Argyris et al. 2010, p. 678):

Definition 1 (Self-Similarity) Let $\delta u(r)$ be a field and $f(\delta u, r)$ its probability density function. Additionally, $\delta \tilde{u}(r)=\lambda^{\zeta} \delta u(\lambda r)$ is a rescaled field with its probability density function $\tilde{f}(\delta u, r)=\lambda^{\zeta} f(\lambda \zeta \delta u, \lambda r) . \delta u(r)$ is self-similar if there exist an exponent $\zeta$ so that for all $\lambda>0$ the probability density functions $f$ and $\tilde{f}$ are identical. So $f$ has to fulfill $f(\delta u, r)=\lambda \zeta f(\lambda \zeta \delta u, \lambda r)$.

Assume that $f(\delta u, r)$ is the probability density function of velocity increments of a fully self-similar turbulent velocity field. Then, without loss of generality, one can write $f$ in terms of an unknown function $g$ as

$$
f(\delta u, r)=\frac{1}{(\varepsilon r)^{\zeta}} g\left(\frac{\delta v}{(\varepsilon r)^{\zeta}}\right)
$$


The probability density function defined this way obeys the demands of selfsimilarity in definition 1 by construction, as one can easily check:

$$
\lambda^{\zeta} f\left(\lambda^{\zeta} \delta u, \lambda r\right)=\lambda^{\zeta} \frac{1}{(\varepsilon \lambda r)^{\zeta}} g\left(\frac{\lambda \zeta \delta v}{(\varepsilon \lambda r)^{\zeta}}\right)=f(\delta u, r)
$$

Inserting equation (1.27) into the relation given in equation (1.23), the behavior of the $\mathrm{n}^{\text {th }}$-order structure function can be expressed as:

$$
S_{n}(r)=\int \delta u^{n} \cdot f(\delta u, r) \mathrm{d} \delta u=\frac{1}{(\varepsilon r)^{\zeta}} \int \delta u^{n} \cdot g\left(\frac{\delta v}{(\varepsilon r)^{\zeta}}\right) \mathrm{d} \delta u
$$

Using the substitution $w=\delta v /(\varepsilon r)^{\zeta}$, this can be further simplified to

$$
\frac{1}{(\varepsilon r)^{\zeta}} \int \delta u^{n} \cdot g\left(\frac{\delta v}{(\varepsilon r)^{\zeta}}\right) \mathrm{d} \delta u=(\varepsilon r)^{n \zeta} \int w^{n} \cdot g(w) \mathrm{d} w=C_{n}(\varepsilon r)^{n \zeta}
$$

In the last step, the integration constant was denoted with $C_{n}$ and is not of interest at this point. The still unknown scaling exponent $\zeta$ can be determined using Kolmogorov's four-fifths law in equation (1.25). As the third-order structure function $S_{3}(r)$ scales as $r^{1}$ in the inertial range, the only possible choice for the order-independent factor $\zeta$ is $1 / 3$, resulting in 


$$
S_{n}(r)=C_{n}(\varepsilon r)^{n / 3}
$$

This expression is one of the central predictions of the K41 framework, as it fully describes the statistics of velocity increments in the inertial range. In principle, the complete probability density function of velocity increments can be calculated using equation (1.30) and equation (1.23) as long as the coefficients $C_{n}$ are known as well.

\subsubsection{Limitations of K41}

In the K41 framework, turbulence is considered to be a self-similar process, with velocity increment probability density functions being preserved over scales. In real turbulence, however, the statistics of the flow depend greatly on the scale. While for large separations, the probability density function of the velocity increments resembles a Gaussian distribution, it develops increasingly heavier tails for smaller separations. In other words, extreme events in turbulence are much more likely to emerge for small separations than for large separations, an effect known as intermittency.

Furthermore, in the derivation of the scaling of structure functions, the energy dissipation rate $\varepsilon$ was assumed to be a global constant. The first to note that the energy dissipation rate is indeed a locally strongly fluctuating quantity were Landau \& Lifschitz (1959). These findings contradict the assumptions in subsection 1.3.2 and led to a refined theory of scaling in turbulence (K62) by Kolmogorov (1962). By 
replacing the constant energy dissipation rate with a log-normal-distributed quantity, one gets an improved prediction for the scaling exponents, now denoted with $\zeta_{n}$, given by the nonlinear function

$$
\zeta_{n}=\frac{n}{3}-\frac{\mu}{18} n(n-3)
$$

The constant parameter $\mu$ in this equation is the so-called intermittency parameter. It is a measure for the deviation from perfect self-similarity. For a review of the current state of theoretical research on intermittency, see section 4.1.

\subsection{The Decay of Turbulence}

Thus far, stationary turbulence has been considered that is stationary in the sense that there is a balance between the amount of energy injected into the system and the amount of energy dissipated at small scales. This balance leads to a statistical stationarity of ensemble and time averages (see section 1.2). Statistical quantities, such as the aforementioned structure functions or energy spectra, are pure quantities of space with no time dependence. However, the situation in many real flows is quite different. Consider again the glass of stirred water as in section 1.1. In the picture of $\mathrm{K} 41$, as long as the stirring continues, an energy cascade exists. The large-scale eddies arising from the stirring will break down into smaller and smaller eddies and will ultimately dissipate into heat. Now consider stopping the stirring. With this, there is no additional energy input in the system anymore, yet the cascade 
process still continues. At some point, the fluid will come to rest, but the statistics of the flow until then are strongly time dependent. The most basic question of interest here is how fast the kinetic energy decays. The first prediction on the rate of decay dates back to de Kármán \& Howarth (1938) who derived a power-law dependence of the turbulent kinetic energy on time, yet weren't able to calculate the exponent of this power-law.

\subsubsection{Kolmogorov's Theory of Decay}

Unlike the statistics predicted by the K41 framework, which only depend on the small and intermediate scales of turbulent motion, the decay of turbulence is governed by the large scales. In the classical description, Kolmogorov (1941c) computed the relation of the energy $E$, dissipation rate $\varepsilon$ and fluctuating velocity $u$ to be independent of Reynolds number:

$$
\frac{\mathrm{d} E}{\mathrm{~d} t}=\frac{3}{2} \frac{\mathrm{d} u^{2}}{\mathrm{~d} t}=-\varepsilon=-C_{\varepsilon} \frac{u^{3}}{L}
$$

Here, $C_{\varepsilon}$ is a Reynolds-number independent constant and $L$ the integral length scale. The isotropic energy spectrum is related to the velocity correlations $\left\langle\mathbf{u} \cdot \mathbf{u}^{\prime}\right\rangle(\mathbf{r})$ with a separation $\mathbf{r}=\mathbf{x}-\mathbf{x}^{\prime}$ by

$$
E(k)=\frac{1}{\pi} \int_{0}^{\infty}\langle\mathbf{u}(\mathbf{x}, t) \cdot \mathbf{u}(\mathbf{x}+(\mathbf{r}, t)\rangle k r \sin (k r) \mathrm{d} r .
$$


For a sufficiently quickly decaying correlation function, this expression can be expanded into a Taylor series for small $k$ and one obtains for the low wavenumber part of the energy spectrum (Davidson 2004, p. 346)

$$
E(k)=\frac{k^{2}}{4 \pi^{2}} \int\left\langle\mathbf{u} \cdot \mathbf{u}^{\prime}\right\rangle \mathrm{dr}-\frac{k^{4}}{24 \pi^{2}} \int r^{2}\left\langle\mathbf{u} \cdot \mathbf{u}^{\prime}\right\rangle \mathrm{dr}+\cdots
$$

The two integrals appearing in this equation are known as the Loitsyanskii integral $\mathcal{I}=\int r^{2}\left\langle\mathbf{u} \cdot \mathbf{u}^{\prime}\right\rangle \mathrm{dr}$ and the Saffman integral $\mathcal{L}=\int\left\langle\mathbf{u} \cdot \mathbf{u}^{\prime}\right\rangle \mathrm{dr}$.

Relying on the finding by Loitsyanskii (1939) that the integral $\mathcal{I}$ is an invariant constant for an isotropic turbulent flow, Kolmogorov (1941c) calculated a relationship between the fluctuating velocity and the integral length scale given by

$$
u^{2} L^{5}=\text { const }
$$

This expression allows for the integration of equation (1.32), resulting in the decay exponent for the turbulent kinetic energy and the integral length scale given by

$$
\begin{gathered}
u^{2} \propto t^{-10 / 7}, \\
L \propto t^{2 / 7} .
\end{gathered}
$$


Note that the constancy of the Loitsyanskii integral $\mathcal{I}$ implies a quickly decaying correlation function such that $\mathcal{L}=0$. As a direct consequence, turbulence of Kolmogorov's type posses a low-wavenumber spectrum of the shape $E(k) \propto k^{4}$. This type of spectrum is generally referred to as the Batchelor spectrum due to the important contributions on the decay of turbulence by Batchelor \& Townsend $(1948 a, b)$

\subsubsection{Saffman's Theory of Decay}

The invariance of the Loiststyanskii integral was questioned and shown to be generally not fulfilled (Proudman \& Reid 1954) to the extent that it is generally divergent (Saffman 1967a). Saffman (1967b) noted that there exists a different invariant in isotropic turbulence, the Saffman integral $\mathcal{L}$. Following an argument analogous to that of Kolmogorov, one can show that the turbulent kinetic energy possesses a different relation between the fluctuating velocity and the integral length scale, as well as a different law of decay, namely

$$
\begin{aligned}
u^{2} L^{3} & =\text { const } \\
u^{2} & \propto t^{-6 / 5}, \\
L & \propto t^{2 / 5} .
\end{aligned}
$$

The low wavenumber part of the energy spectrum can be shown to grow as $E(k) \propto k^{2}$. All these results concern decaying turbulence at high Reynolds numbers, 
hence known as the initial period of decay. For very large times, where the Reynolds number becomes small, the exponent of the power-law increases. Viscous effects begin to dominate the dynamics, resulting in a predicted decay rate for the final period of decay of $u^{2} \propto t^{-2.5}$ for Kolmogorov turbulence and $u^{2} \propto t^{-1.5}$ for Saffman turbulence (Batchelor \& Townsend 1948b).

\subsubsection{Physical Picture}

It is possible to relate the existence of the decay invariants to the internal structure of turbulence (Landau \& Lifschitz 1959, Saffman 1967b, Davidson 2004). In the frame of Kolmogorov turbulence, Landau considered a patch of turbulence of Volume $V$ with a net angular momentum $\mathbf{H}$ and vanishing linear momentum $\mathbf{L}$. The latter one can be constructed by limiting the patch of turbulence to a closed domain, which enforces $\mathbf{L}=\int \mathbf{u} \mathrm{d} V=0$. It can be shown that the angular momentum of the turbulent patch,

$$
\mathbf{H}=\int_{V} \mathbf{x} \times \mathbf{u} \mathrm{d} V
$$

can be directly related to Loitsianskii's integral $\mathcal{I}$ via

$$
\frac{\left\langle\mathbf{H}^{2}\right\rangle}{V}=-\int\left\langle\mathbf{u} \cdot \mathbf{u}^{\prime}\right\rangle \mathrm{dr}=\mathcal{I}
$$


Assuming $\mathcal{I}$ to be an invariant, Kolmogorov's theory corresponds to an underlying structure of turbulent patches carrying significant angular moment but negligible linear momentum. For Saffman's theory, one can show that it is possible to rewrite the Saffman integral by exchanging volume and ensemble averages as

$$
\mathcal{L}=\int\left\langle\mathbf{u} \cdot \mathbf{u}^{\prime}\right\rangle \mathrm{dr}=\frac{1}{V}\left\langle\left[\int \mathbf{u} \mathrm{d} V\right]^{2}\right\rangle
$$

This conserved quantity is a measure of the net linear momentum of the turbulent patch. Depending on whether the patch carries a significant amount of linear momentum, the Saffman integral $\mathcal{L}$ becomes non-vanishing. Note that a nonvanishing Saffman integral automatically enforces a divergence of the Loitsianskii integral. Turbulence in Saffman's theory, therefore, consists of patches carrying nontrivial amounts of linear momentum with vanishing angular momentum (see figure 1.3). 


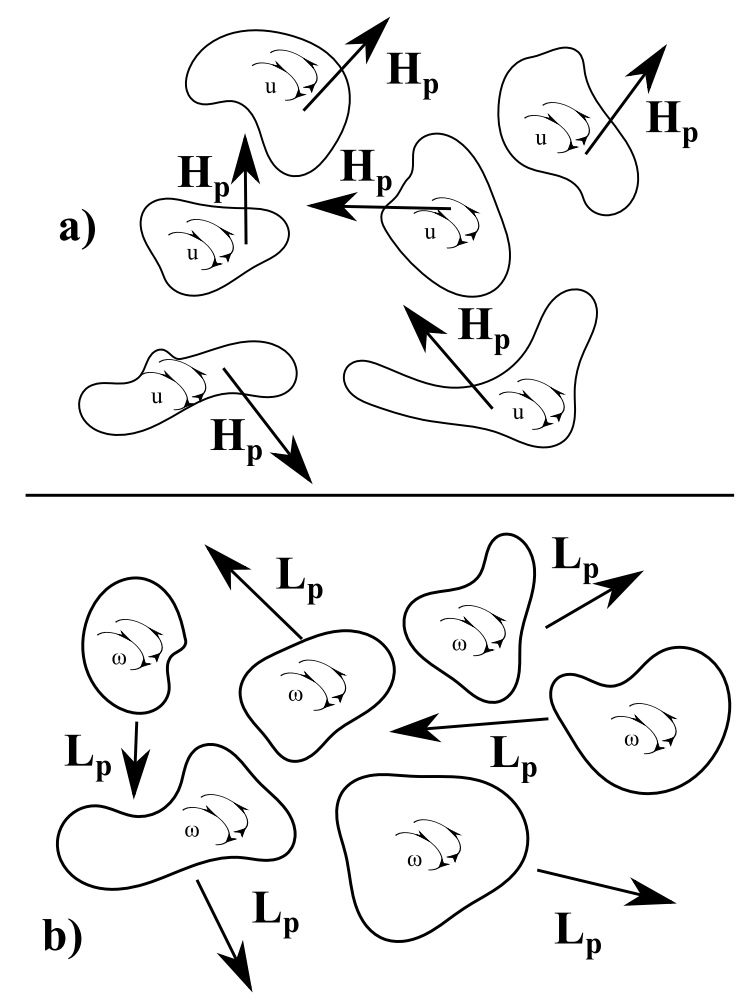

Figure 1.3: a) In the picture of Batchelor, turbulence consists of patches carrying a significant amount of angular momentum $\mathbf{H}_{\mathbf{p}}$ and negligible amount of linear momentum. b) Saffman's theory corresponds to turbulence of patches of net linear momentum $\mathbf{L}_{\mathbf{p}}$ with virtually vanishing angular momentum. (The sketch is following Davidson (2004).) 


\section{Experimental Methods}

The results of this thesis are based upon turbulence data obtained in two different wind tunnels. The data from the Variable Density Turbulence Tunnel (VDTT) at the Max-Planck-Institute for Dynamics and Self-Organization makes up the major part. Additional velocity time series were obtained in the scope of the ESWIRP project, "Investigation of the small-scale statistics of turbulence in S1MA", at the ONERA wind tunnel in Modane, France. The Göttingen facility is described in section 2.1 and the Modane facility in section 2.2. All turbulence data has been collected by means of a classical measurement technique called hot-wire anemometry. The

details of this technique and of the Nano-Scale Thermal Anemometry Probes (NSTAPs) used are given in section 2.3. In section 2.4, the individual datasets and respective experimental setups are described. Parts of section 2.1 have been published in the Review of Scientific Instruments in greater detail (Bodenschatz, Bewley, Nobach, Sinhuber \& Xu 2014). 


\subsection{The Variable Density Turbulence Tunnel}

At high Reynolds numbers, turbulence is assumed to exhibit universal features (see chapter 1), such as predictable scale separation and the development of an inertial range. To investigate the statistical behavior of turbulent flows at high Reynolds numbers, one could directly measure natural, atmospheric flows. These flows tend to possess extremely high Reynolds numbers of $R_{\lambda} \sim\left(10^{4}\right)$ (Siebert et al. 2006). However, one would also like to have precisely controlled conditions for the flow, which is only possible to a very limited extent in natural flows. The question, how the Reynolds number $R e=U L / v$ determines the statistical behavior of a turbulent flow in a given geometry, can not in particular be answered from the in situ observation of atmospheric flows alone. Wind tunnels provide an important experimental tool for producing nearly homogeneous, isotropic turbulence, despite being unable to achieve atmospheric Reynolds numbers. Limits to the mean speed and the length scales arise from the construction and operational costs of the experiments. To balance the need for high Reynolds numbers and well-controlled conditions, one strategy is to build an extremely large wind tunnel like the S1MA in Modane. This comes at the disadvantage of steep operational costs and inflexibility of the experimental setup, being limited to the use of air. The second approach is to make use of pressurized gases in a smaller wind tunnel. Since the dynamic viscosity $\mu$ of a gas only weakly depends on pressure, the kinematic viscosity $v=\mu / \rho$ can be adjusted over a wide range by changing the density, i.e. pressure, of the gas. Using heavy gases at high pressures, one can obtain high Reynolds numbers in a wind 
tunnel of moderate size, the approach chosen with the VDTT.

\subsubsection{Historical review of pressurized wind tunnels ${ }^{1}$}

For over a century, pressurized wind tunnels have proven to be important tools in researching aerodynamic questions because of their ability to independently adjust flow Reynolds number and Mach number by independently changing the pressure and mean speed of the working fluid. Without the possibility of running numerical simulations on computers, conducting wind tunnel experiment was the only way to test small-scale aerodynamic models before production. Even today, the limited computational power of even the most modern computers still necessitates the testing of models in wind tunnels at high Reynolds numbers. The first wind tunnel in which the working gas could be pressurized to adjust the Reynolds number was the "Variable Density Wind Tunnel of the National Advisory Committee for Aeronautics" (VDT) built by Munk (1921) at the Langley Research Center in Virginia. This tunnel, finished in 1923, was able to pressurize air up to 21 bar, reaching Reynolds numbers $R e_{W T}=0.1 \sqrt{A} U / v=5.4 \cdot 10^{6}$ based upon the cross section of the tunnel $A$ and the mean speed $U$ (Munk \& Miller 1926). This wood recirculating tunnel of the Göttingen type (Oswatitsch \& Wieghardt 1987) was destroyed in a fire in 1927 and rebuilt in 1930 (Jacobs \& Abbot 1933). Because it produced high turbulence intensities, the limitations of the flow quality in the VDT were deemed to be too severe. This lead to the design of the $44.5 \mathrm{~m}$ long "Langley

${ }^{1}$ This subsection follows the longer historical review in Bodenschatz, Bewley, Nobach, Sinhuber \& $\mathrm{Xu}$ (2014). 
two-dimensional low-turbulence pressure tunnel" in 1938, which reached $R e_{W T}=$ $6.1 \cdot 10^{6}$ using of compressed air at 10 bar (von Doenhoff \& Abbott 1947). The tunnel successfully provided a high quality aerodynamic research tool for decades (McGhee et al. 1984, Choudhari et al. 2002) until demolition began in $2006^{2}$. The first variable density turbulence tunnel built in Europe was the "Compressed Air Tunnel" at the National Physical Laboratory in Taddington in 1931 (Pankhurst 1972). Using compressed air at 25 bar, the tunnel reached $R e_{W T}=8 \cdot 10^{6}$. In Germany, the first low pressure variable density tunnel was built at the Deutsche Forschungsanstalt für Luftfahrt in 1956 (Schlichting 1956). The "Variable density high speed cascade wind tunnel" was able to operate at pressures between 0.1 bar and 1 bar air to reach Reynolds numbers up to $R e_{W T}=4 \cdot 10^{6}$. Despite the numerous high-quality, variable-density turbulence tunnels built and operated over the span of 40 years, essentially none were used to conduct fundamental turbulence research. The first reported study on the topics of turbulence produced by a classical grid was published by Kistler \& Vrebalovich (1966). The authors used the immense "Southern California Co-operative Wind Tunnel" before its closing (Millikan et al. 1948). After the initial results from Kistler and Vrebalovich, several pressurized wind tunnels were built to focus on fundamental turbulence questions. In Jülich, a high-pressure wind tunnel running Helium at 40 bar was used to investigate the flow behind spheres at $R e_{W T}=3.1 \cdot 10^{5}$ (Achenbach 1972). At the German Aerospace Center in Göttingen, the "High Pressure Wind Tunnel" operating at air up to 100 bar was constructed and, e.g., used to investigate the flow around

\footnotetext{
${ }^{2}$ See http://crgis.ndc.nasa.gov/historic/Low_Turbulence_Pressure_ Tunnel (As of 06.02.2015, 10:20) for a history of the Low Turbulence Pressure Tunnel.
} 
cylinders (Försching et al. 1981). The most recent variable density tunnels before the construction of the VDTT were the Princeton/DARDPA-ONR SuperPipe Facility and the Princeton/ONR High Reynolds Number Testing Facility (Zagarola \& Smits 1997). These facilities run with air pressurized to over 200 bar, reaching Reynolds numbers of up to $R e_{W T}=9.6 \cdot 10^{6}$ in the latter case. In 2009, the Variable Density Turbulence Tunnel was inaugurated at the Max Planck Institute for Dynamics and Self-Organization. The key concept in this tunnel was to use pressurized SulfurHexafluoride as a working gas, reaching Reynolds numbers up to $\operatorname{Re}_{W T}=4.4 \cdot 10^{6}$ in a relatively small tunnel at low mean speeds (Bodenschatz et al. 2014). The technical details of this tunnel and of the turbulent flow within, from which the major part of the data of this thesis stems from, will be covered in the following subsections.

\subsubsection{Geometric Details}

The VDTT is is a pressure vessel capable of being pressurized up to 15 bar with non-combustible gases. Using of Sulfur Hexafluoride $\left(\mathrm{SF}_{6}\right)$ as a working gas allows for adjustable and extremely high Reynolds numbers due to its high density relative to air. As the kinematic viscosity, $v$, for most gases is inversely proportional to their density, one is able to adjust the Reynolds number by changing the pressure of the gas without changing the mean speed of the flow or the tunnel geometry. At 15 bar, $\mathrm{SF}_{6}$ reaches roughly one tenth of the density of water, whereas at 1 bar it is still 5 times denser than air (see table 2.1). This way, variations of two orders of magnitude in Reynolds number can be achieved without changing the mean speed 
of the gas. At the same time, due to the relatively small diameter and wind speed of the VDTT in comparison to an hypothetical air wind tunnel operating at the same Reynolds number, the operational costs can be kept low. Additionally, the moderate flow parameters facilitate the use of Lagrangian measurement techniques.

\begin{tabular}{c|c|c|c} 
Gas & $p[$ bar $]$ & $\rho\left[\frac{\mathrm{kg}}{\mathrm{m}^{3}}\right]$ & $v\left[\frac{\mathrm{m}^{2}}{\mathrm{~s}}\right] \cdot 10^{-7}$ \\
\hline Air & 1 & 1.2 & 152 \\
$\mathrm{SF}_{6}$ & 1 & 6.1 & 24.8 \\
$\mathrm{SF}_{6}$ & 2 & 12.3 & 12.2 \\
$\mathrm{SF}_{6}$ & 4 & 25.2 & 5.99 \\
$\mathrm{SF}_{6}$ & 8 & 53.5 & 2.86 \\
$\mathrm{SF}_{6}$ & 15 & 114.3 & 1.39
\end{tabular}

Table 2.1: Pressure and viscosity of the working gases at selected pressures and $20^{\circ} \mathrm{C}$, estimated from the experiments by Hoogland et al. (1985)

Albeit non-toxic, $\mathrm{SF}_{6}$ is a strong greenhouse gas that is damaging for the environment and, being heavier than air, it can lead to suffocation. The VDTT is constructed as a recirculating Göttingen-type wind tunnel, keeping the working gas in a closed loop. This design conserves energy and allows to record arbitrarily long datasets.

The VDTT is a $18.2 \mathrm{~m}$ long and $5.3 \mathrm{~m}$ tall stainless steel high-pressure vessel with a total volume of $88 \mathrm{~m}^{3}$ (see figure 2.1). The two straight sections, the upper one containing the test section, have inner diameters of $1.84 \mathrm{~m}$ and a circular cross sections. The elbows have an inner diameter of $1.54 \mathrm{~m}$. The fan (covered in subsection 2.1.3) is located at the downstream position of the lower straight section and there is a heat exchanger that is responsible for the temperature control (see subsection 2.1.4) at the upstream end of the upper straight section. The heat 


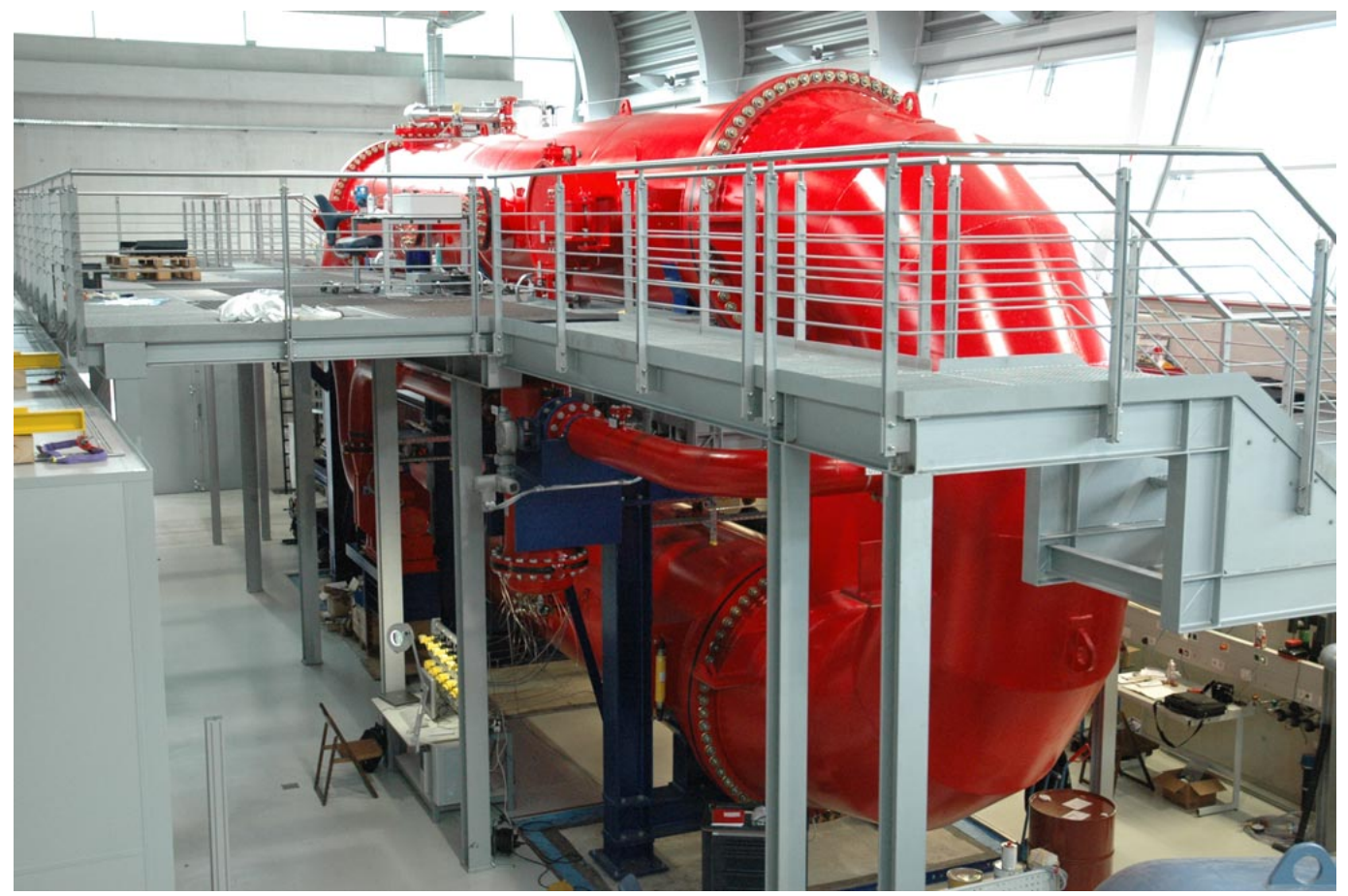

Figure 2.1: Photograph of the Variable Density Turbulence Tunnel

exchanger is $1.27 \mathrm{~m}$ wide and $0.96 \mathrm{~m}$ high. Extensive details on the construction, gas handling system, safety systems and filter bypass can be found in Bodenschatz, Bewley, Nobach, Sinhuber \& Xu (2014).

Downstream of the heat exchanger, the inner cross section is expanded to a height of about $1.5 \mathrm{~m}$ and a width of $1.3 \mathrm{~m}$ with cut edges to form a roughly octagonal shape (see figure 2.3). The 8.8-meter-long measurement section is separated from the round inner tunnel walls by steel sheets with plexiglass windows. Behind these inner walls, cables, tubes as well as supplementary electronics are lead to the downstream end of the measurement section where the measurement probes are located (see figure 2.2). For a description of the individual setups, see section 2.4. 


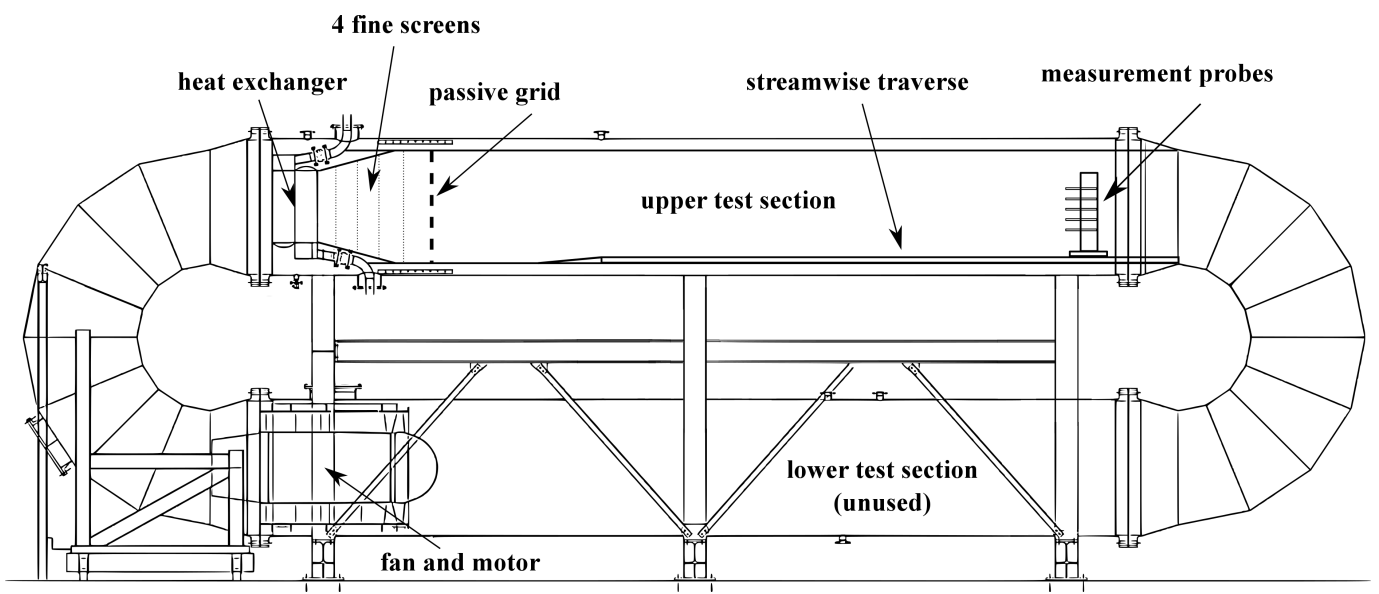

Figure 2.2: Simplified sketch of the Variable Density Turbulence Tunnel, also see Bodenschatz, Bewley, Nobach, Sinhuber \& Xu (2014)

\subsubsection{Turbulence Production}

The working gas is set in motion by means of a 20-blade fan driven by a $210 \mathrm{~kW}$ electric motor with a $40 \mathrm{~kW}$ water cooling system. The fan and the motor are located at the end of the lower straight section and are able to produce constant mean flow velocities between $0.5 \mathrm{~m} / \mathrm{s}$ and $5.3 \mathrm{~m} / \mathrm{s}$. Turbulence is produced at the upstream end of the upper test section by a classical bi-planar grid of crossed rectangular bars (see figure 2.4). This is classical in the sense that grids of crossed bars have been used frequently throughout the last century to produce turbulence of low intensity and high flow quality concerning isotropy and homogeneity (Simmons \& Salter 1934, Taylor 1935, Dryden et al. 1937, Corrsin 1942, Batchelor \& Townsend 1947, Comte-Bellot \& Corrsin 1966, Kistler \& Vrebalovich 1966). The incoming flow is disturbed by the stationary bars, producing turbulent wakes that extend downstream. As a rule of thumb, turbulence has developed into a homogeneous and isotropic state, 


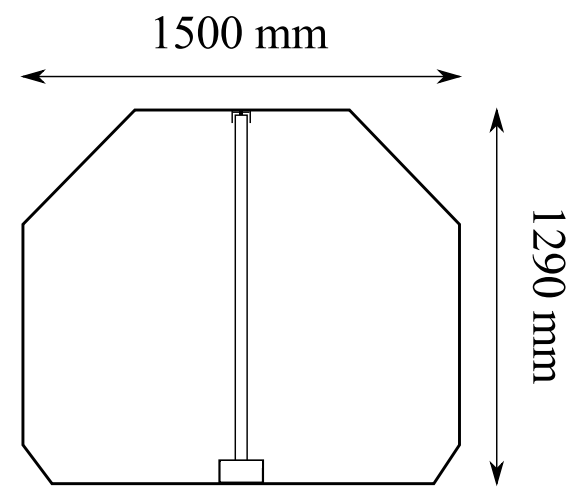

Figure 2.3: Simplified schematic of the cross section of the test section, see also Bodenschatz, Bewley, Nobach, Sinhuber \& Xu (2014)

20 mesh lengths downstream of the grid, provided the absence of shear. For the majority of the experiments, the mesh spacing of the grid was $180 \mathrm{~mm}$ with a grid bar diameter of $40 \mathrm{~mm}$, blocking $38 \%$ of the cross section of the tunnel. For several experiments, a smaller grid with a mesh spacing of $106.6 \mathrm{~mm}$ was installed in the tunnel. Details on modifications to the grid geometry in several special experiments can be found in section 2.4.

One of the main advantages of using a passive grid, apart from flow quality, simplicity of construction and ease of maintenance, is that the produced turbulence has remarkably low turbulence intensities $u^{\prime} / U$, making it possible to apply Taylor's Frozen Flow Hypothesis to convert temporal data into spatial information (see subsection 1.2.4). Once generated by the grid bars, there is no external energy input into the downstream-swept turbulence and it freely decays as described in section 1.4. Table 2.2 gives a short summary of the basic flow parameters of a few selected experiments using $\mathrm{SF}_{6}$. 


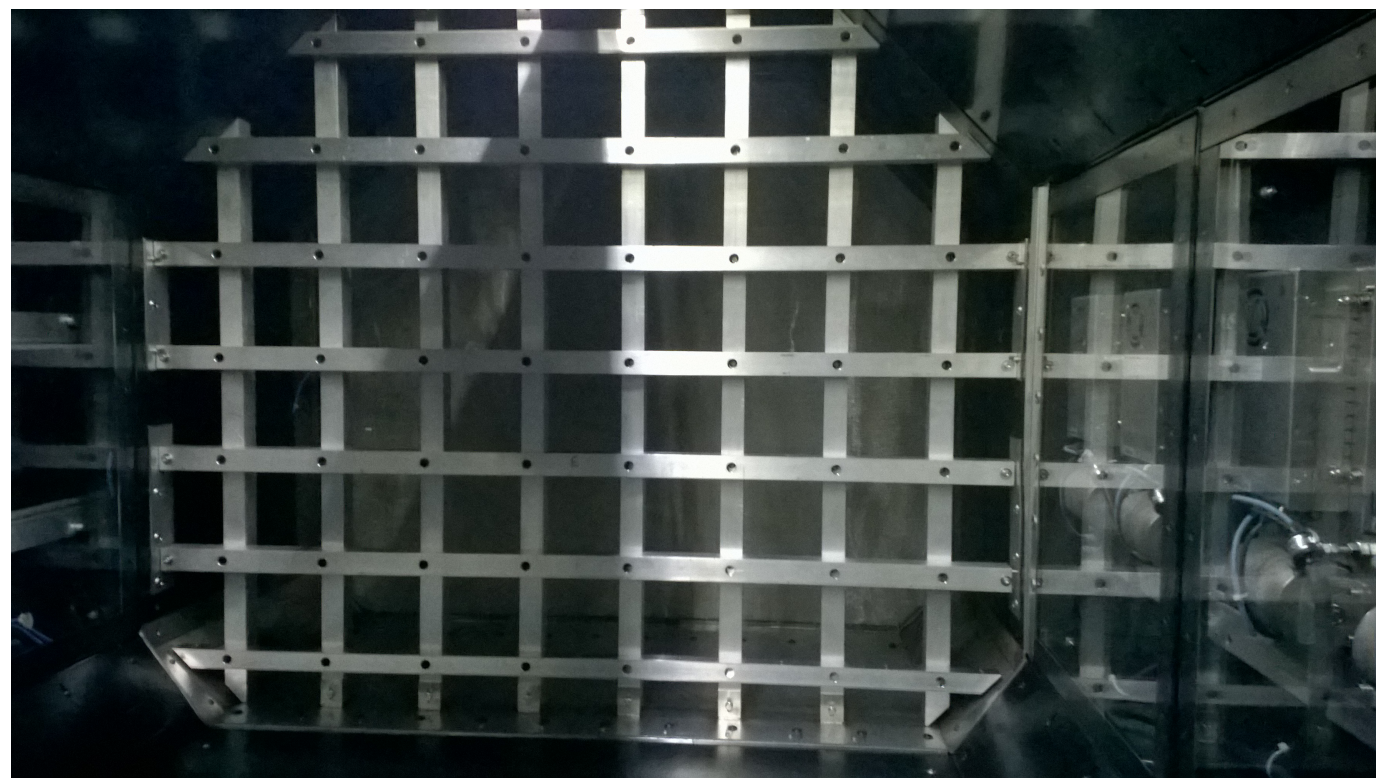

Figure 2.4: Photograph of the-turbulence generating grid, viewing upstream.

With the use of $\mathrm{SF}_{6}$, the Reynolds numbers obtainable in the VDTT exceed those of any comparable experiment, including the highest Reynolds number classical grid experiment by Kistler \& Vrebalovich (1966). Note that the increase in Reynolds number and the scale separation manifest through a shrinking of the small scales, as expected for turbulence generated by a passive grid. The integral length scale and turbulence intensity is mainly fixed by the geometry of the grid. Increasing the pressure and thus the Reynolds number, therefore, leads to smaller and smaller Kolmogorov scales. It is on one hand advantageous that the large-scale structures of the flow remain essentially constant between experiments at different conditions, but it is disadvantageous on the other, as resolving the smallest structures in the flow demands for highly specialized measurement techniques to be covered in section 2.3 . 


\begin{tabular}{cl|c|c|c|c|c} 
Pressure & $p[\mathrm{bar}]$ & 2.5 & 6.5 & 8 & 12 & 14.5 \\
\hline Mean speed & $U[\mathrm{~m} / \mathrm{s}]$ & 4.12 & 4.20 & 4.20 & 4.32 & 4.33 \\
Turbulence intensity & $u^{\prime} / U$ & $3.04 \%$ & $3.09 \%$ & $3.12 \%$ & $3.13 \%$ & $3.11 \%$ \\
Integral length scale & $L[\mathrm{~mm}]$ & 123.9 & 127.0 & 128.2 & 130.0 & 129.5 \\
Kolmogorov length scale & $\eta[\mu \mathrm{m}]$ & 91 & 43 & 36 & 25 & 22 \\
Reynolds number & $R_{\lambda}$ & 500 & 880 & 1000 & 1300 & 1450
\end{tabular}

Table 2.2: Basic flow parameters for a few selected experiments.

\subsubsection{Temperature Control ${ }^{3}$}

All mechanical energy injected into by the motor into the turbulent flow is dissipated into heat. In order to ensure temperature stability, a $280 \mathrm{~kW}$ heat exchanger is installed at the upstream end of the upper test section. It consists of two sets of water-cooled plates. The cooling water is supplied by the institute's cooling water system at a constant flow rate. Temperature control is accomplished via a proportional-integral-derivative (PID) controller that mixes the cooling water with the warm return flow water from the heat exchanger.

The cooling system automatically turns on when the fan is started. After an initial adjustment time, the length of which depends on the working gas and its pressure (usually of the order of several tens of minutes for air at atmospheric pressure and of a few minutes for $\mathrm{SF}_{6}$ at high pressures), the PID controller stabilizes the temperature of the mean flow with an accuracy of about $\pm 0.15 \mathrm{~K}$. A typical temperature time series can be seen in figure 2.5. The oscillation in the mean temperature with a period of approximately 2 minutes is slower than any observable

\footnotetext{
${ }^{3}$ The thermocouple data presented in this subsection was sampled at $40 \mathrm{~Hz}$ using a $0.076 \mathrm{~mm}$ thermocouple housed in a ceramic insulation. It was done with the gracious help of Holly Capello, who built the probes, wrote the measurement code and supported the measurement process.
} 


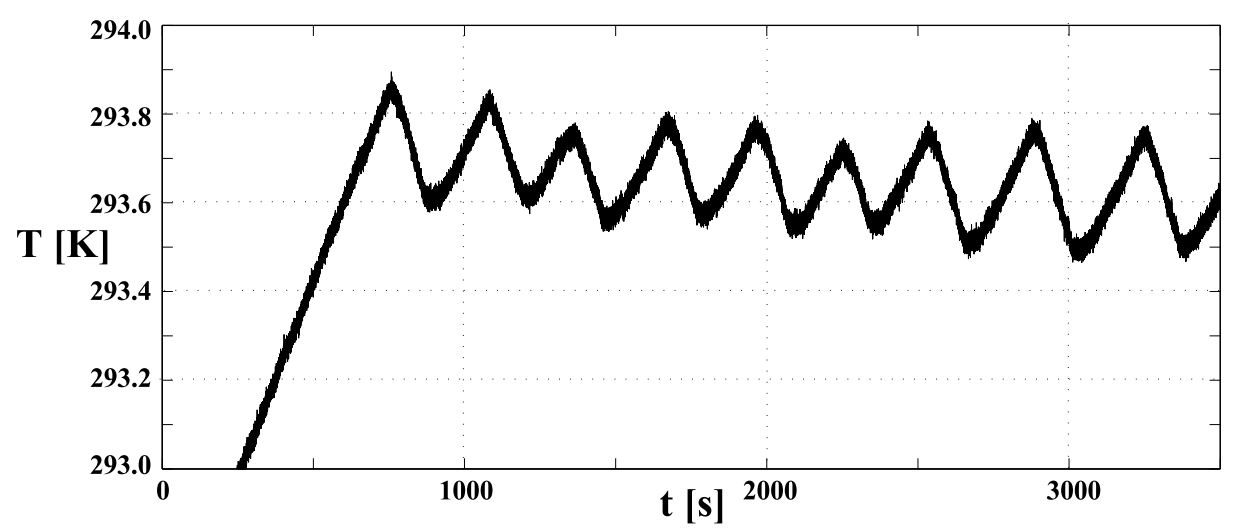

Figure 2.5: Time series of temperature information at the end of the measurement section at the centerline. The fan was turned on at $t<0$. The data was obtained in air at 1 bar.

turbulent signal and does therefore not influence the measurements in a significant way. The data shown was obtained in air and thus at low motor power consumption. The initial adjustment time decreases drastically when gases of higher density are used. The experimental protocol is such that no data is taken before the PID controller has settled into a controlled state.

As discussed in section 2.3, hot-wire probes react sensitively to temperature changes. If the fluctuations in the temperature are either too large or too fast, distinguishing between turbulent velocity signals and temperature fluctuations becomes difficult. Figure 2.6 shows the probabilty distribution function of velocity fluctuations. Once the oscillations in the data from figure 2.5 are removed using a high-pass software filter, one obtains the distribution of the instantaneous temperature fluctuations. These fluctuations turn out to be Gaussian distributed and an order of magnitude smaller than the mean-temperature-stability quality for the core part 


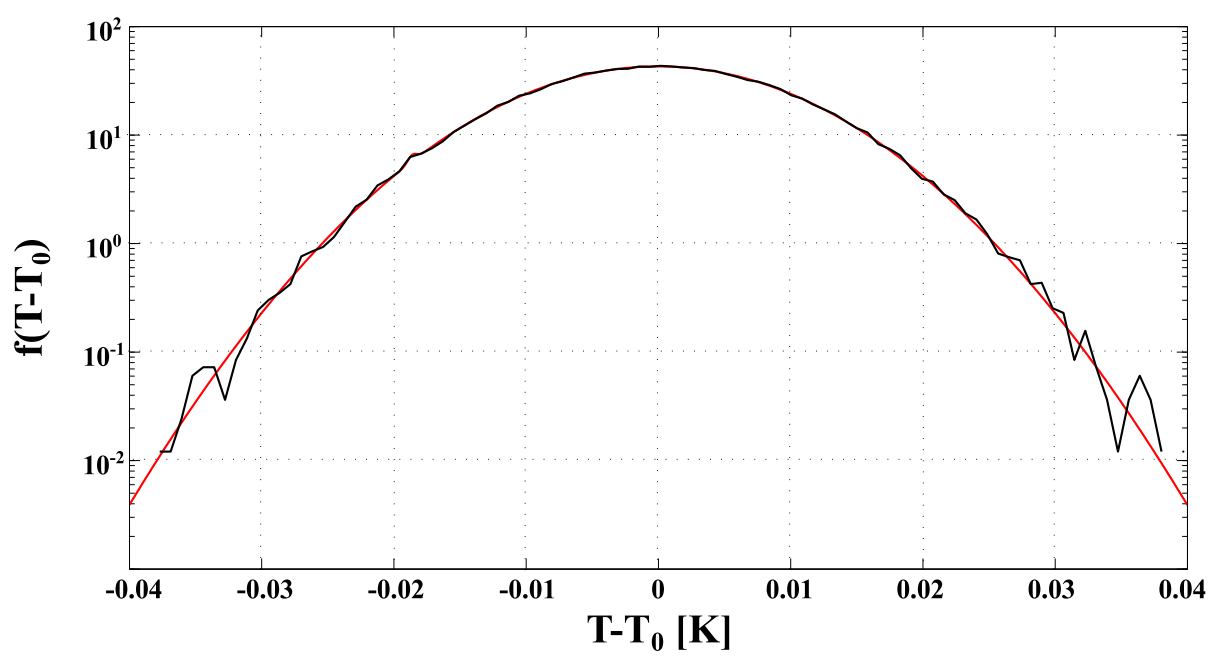

Figure 2.6: Probability distribution function of the temperature deviations $T-T_{0}$ from the mean temperature, $T_{0}$. The same data as in figure 2.5 was restricted to the time after the PID controller settled into a controlled state; the slow temperature drifts were removed by a software high-pass filter to obtain information about the temperature fluctuations. A Gaussian fit to the black data is given in red.

of the distribution. The outer tails of the distribution that concern events four orders of magnitude more unlikely than events in the core part of the distribution functions still cover temperature events only half as large as the mean temperature stability of $0.1 \mathrm{~K}$. Even for small temperature deviations, there might be the possibility of an intermittent distribution of temperature increments with heavy tails which will influence the measurement of the statistics of the extreme turbulent events in the velocity statistics. Therefore, it is imperative to verify the smallness of temperature increments. In figure 2.7 , the temperature increment probability density functions for different time increments $\tau$ are shown. The smallest time increment measured here, $\tau=0.025 \mathrm{~s}$ corresponds to structures of the size of $1.6 \mathrm{~cm}$ and, thus, to inertial 
range structures. Independent of the increment, the probability density function does not show measurable intermittent effects. The measured probability of extreme events drops off quickly, ensuring that the temperature fluctuations in the VDTT do not influence the velocity measurements. In principal, intermittent effects in the distribution of temperature increments are expected (Warhaft 2000). However, from the results from figure 2.7 , a temperature increment of $0.01 \mathrm{~K}$ would translate into a velocity increment of $10^{-8} \mathrm{~m} / \mathrm{s}$. The temperature effects can, thus, be assumed to be smaller than the accuracy of the measurement device and, as a consequence, do not influence the velocity measurements in the VDTT.

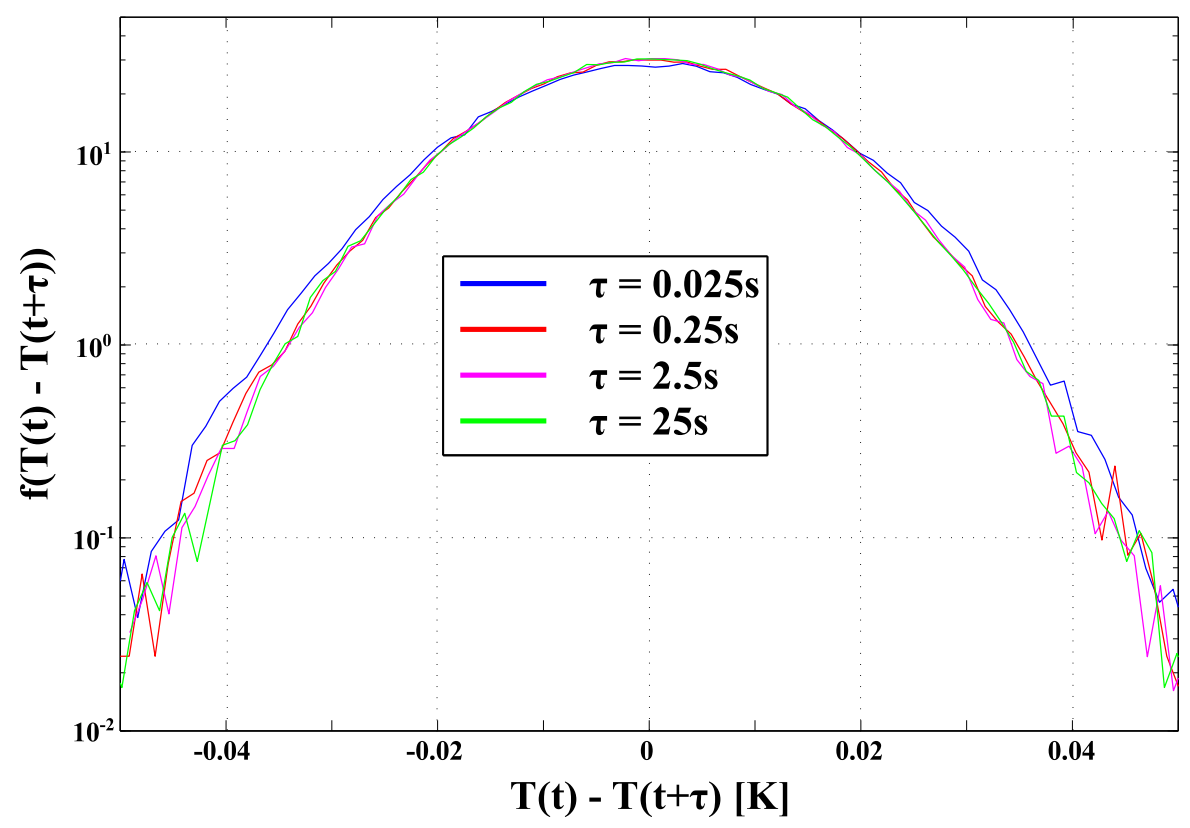

Figure 2.7: Probability distribution function of the temperature increments.

The long time temperature stability of the tunnel is also maintained by the cooling 
system over arbitrary times. Figure 2.8 shows the evolution of the mean temperature in the VDTT obtained with a standard Dantec Dynamics temperature sensor in a flow at 15 bar of $\mathrm{SF}_{6}$. The drift in the mean temperature is negligible as it is smaller than the fluctuations of the temperature themselves. This negligibility is ensured by the results from figure 2.13.

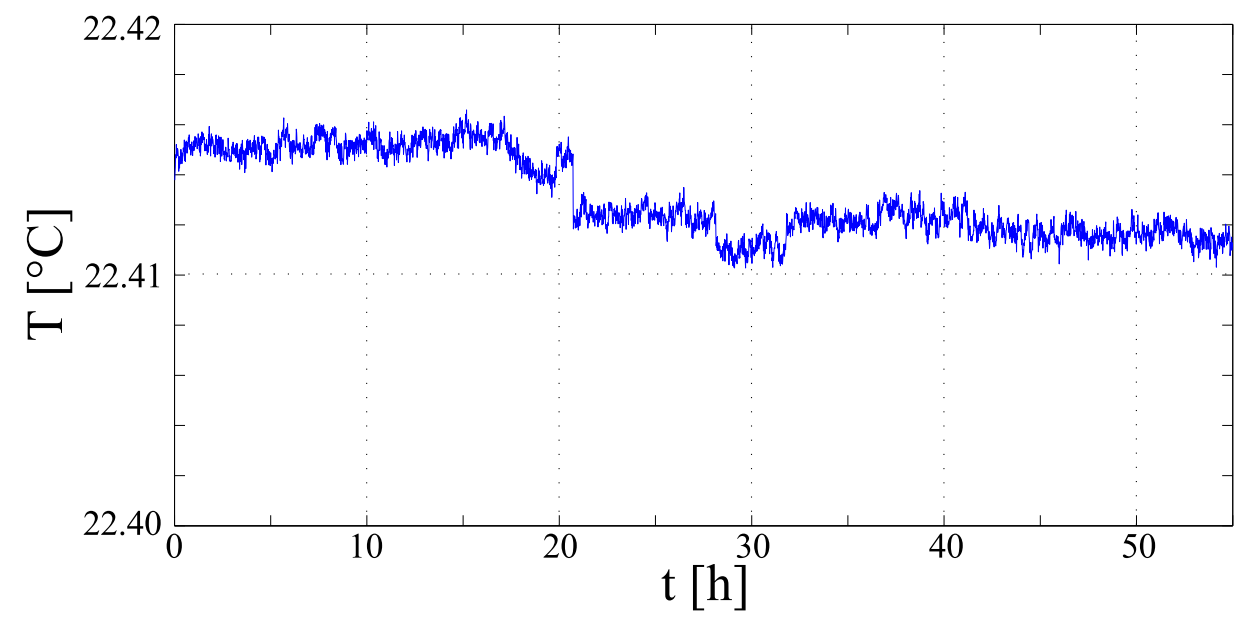

Figure 2.8: Long time evolution of the mean temperature in the tunnel for a measurement at 15 bar of $\mathrm{SF}_{6}$. Shown is the temperature measured with a Dantec Dynamics, averaged over $50 \mathrm{~s}$ intervals, as a function of time. There is only a negligible trend in the mean temperature.

\subsubsection{Flow Properties}

As discussed in chapter 1 , the fundamental study of homogeneous, isotropic turbulence is considered here. With that in mind, great effort was undertaken to improve the properties on the flow to the best possible extent (Bodenschatz, Bewley, Nobach, Sinhuber \& Xu 2014). These measures include, among others, the fin design of the heat exchanger to remove possible large scale Görtler vortices, stemming from 
the flow passing the curvature of an elbow (Görtler 1940, Hawthorne 1951), the inclusion of four meshes with fine grid spacing in the expansion behind the heat exchanger to homogenize the flow (Schubauer \& Spangenberg 1948, Laws \& Livesey 1978), as well as a slightly inclinated test section to counteract growing boundary layers. To further improve the homogeneity of the flow, the width of the top and bottom grid bars were reduced by $4 \mathrm{~mm}$. The resulting turbulent flow at the end of the test section has a virtually constant mean flow profile in the bulk of the wind tunnel with a constant turbulence intensity profile, negligible shear and a low, but in grid turbulence unavoidable (Lavoie et al. 2007), isotropy ratio ${ }^{4}$ of less than 1.1. It thus provides a flow quality comparing favorably to the most sophisticated experiments (see e.g. Comte-Bellot \& Corrsin (1966), Lavoie et al. (2007) or Krogstad \& Davidson (2010)). Further details on the flow properties can be found in Bodenschatz, Bewley, Nobach, Sinhuber \& Xu (2014).

\subsection{The S1MA}

While most of the data presented in this thesis stems from the VDTT, some experiments were conducted at an atmospheric wind tunnel in Modane, France. The S1MA is an atmospheric open wind tunnel operated by ONERA, the french aerospace $1 a b^{5}$.

This gigantic wind tunnel has an outer length of $155 \mathrm{~m}$ and a width of $40 \mathrm{~m}$. The flow is driven by two fans of a total of $88 \mathrm{MW}$, which are directly driven mechanically by hydro power via Pelton turbines. The test section has an inner

\footnotetext{
${ }^{4}$ The isotropy ratio is defined as the ratio of streamwise to transverse velocity fluctuations.

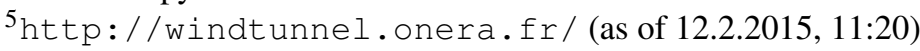




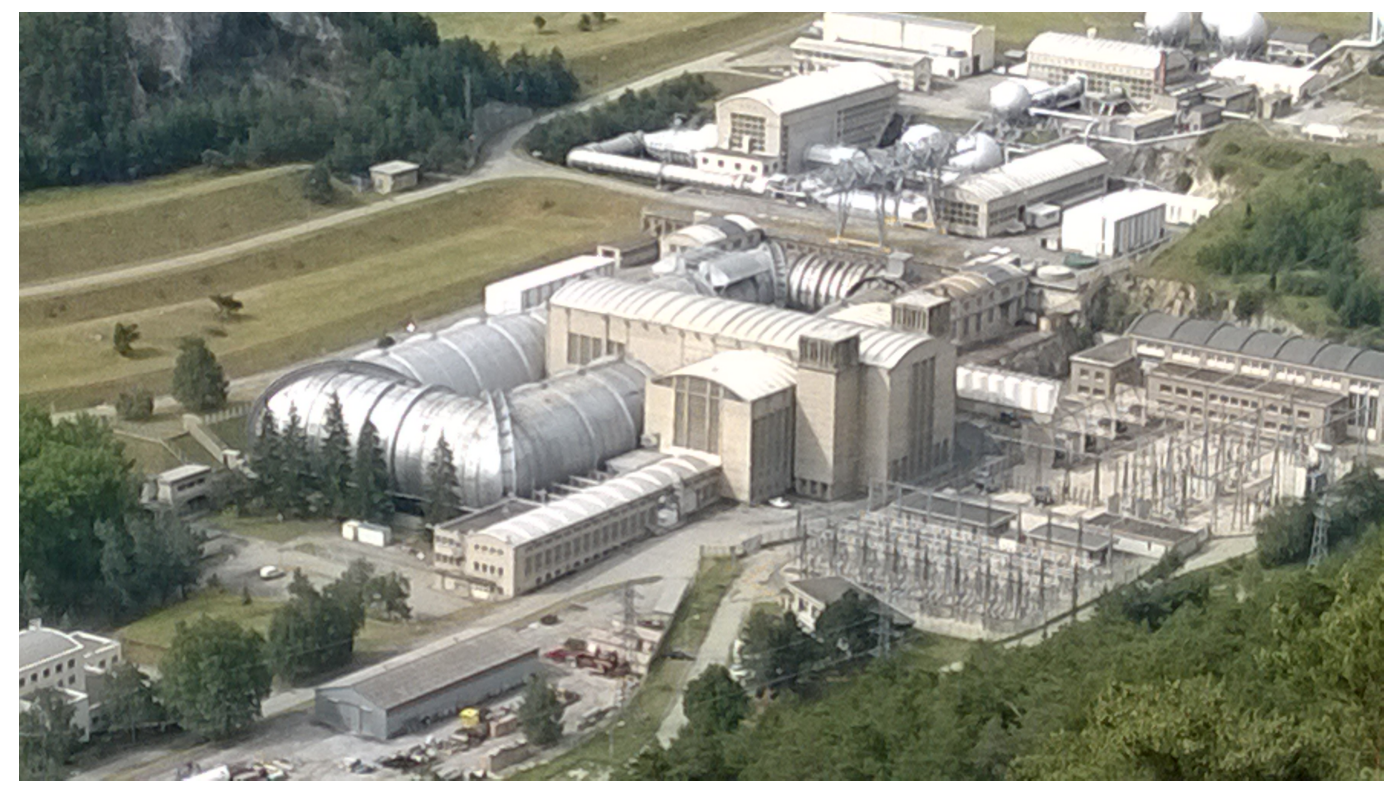

Figure 2.9: Photograph of the S1MA wind tunnel at ONERA, the French aerospace lab, located in Modane, France.

diameter of $8 \mathrm{~m}$, allowing for the testing of large-scale models. In contrast to the VDTT, the S1MA is designed to reach high wind speeds up to Mach numbers of 1, focusing on aerospace engineering studies. Figure 2.9 shows a photograph of the wind tunnel and experimental building. Unfortunately, photography is strictly prohibited on-site and inside the tunnel due to confidentiality regulations, so no further pictures can be provided here. A sketch of the facility is shown in figure 2.10 .

Being designed for industrial applications, the S1MA is not usually available for scientific purposes, mainly due to the steep operational costs of the rentable wind tunnel. The experiments presented here were made possible in the scope of the European Project FP 7: European Strategic Wind tunnels Improved Research 


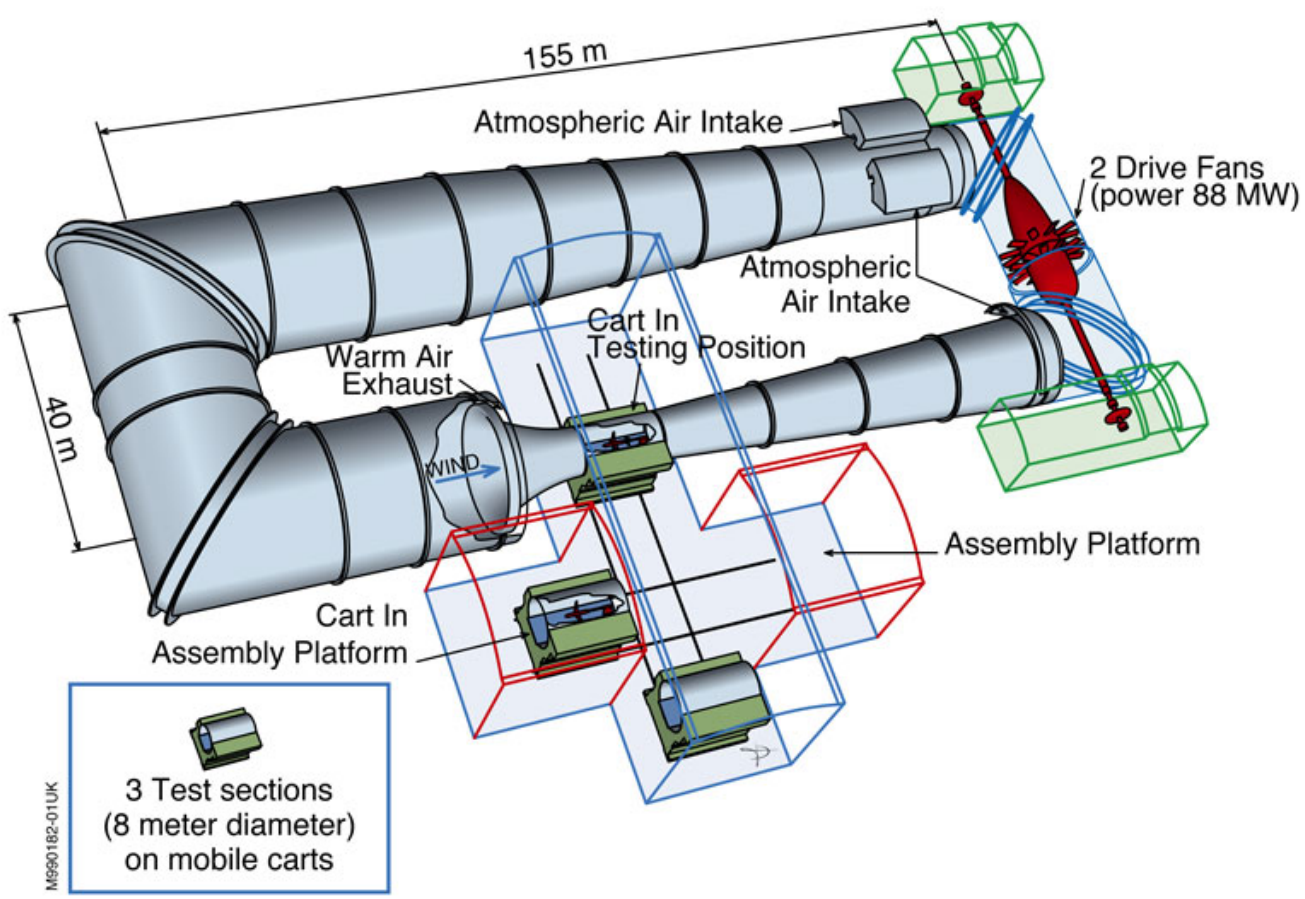

Figure 2.10: Sketch of the S1MA. Taken from ONERA's homepage http://windtunnel.onera.fr/sites/windtunnel.onera. fr/files/illustrations/S1MA-aerodynamic-circuit.jpg (as of 08.04.2015, 15:20).

Potential (ESWIRP $\left.{ }^{6}\right)$. In the project "Investigation of the small-scale statistics of turbulence in S1MA", an inflatable grid with a diameter of $10 \mathrm{~m}$ and a mesh spacing of $0.625 \mathrm{~m}$, blocking $34.7 \%$ of the cross-section, was installed in the contraction upstream of the test section to produce homogeneous, isotropic turbulence. The aim of this campaign was to investigate the small scales of turbulent motion with the greatest possible variety of measurement techniques. Due to the size of the grid and the test section, the small scales were expected to be measurable without spatial

\footnotetext{
${ }^{6}$ http: / / www.eswirp.eu/ (as of 12.02.2015, 11:30)
} 
filtering effects. The experiments, in which I participated along with over 30 other scientists with over a dozen different measurement techniques, were conducted in the week between July $7^{\text {th }}$ and July $11^{\text {th }}, 2014^{7}$. By the time of submission of this thesis, the ESWIRP project had concluded ${ }^{8}$ and a database with the collected data from all collaborators was being created, making the processed experimental data publicly accessible by the $1^{\text {st }}$ of September, 2017.

\subsection{Hot-Wire Anemometry}

Since the beginning of the 20th century, hot-wires were to become an important tool for measuring mean wind velocities and, with the advent of adequate electronics from the 1920s on, for the precise measurement of velocity fluctuations (see ComteBellot (1976)). Over the decades, the quality of the probes themselves and that of the supporting electronics has improved immensely, making thermal anemometry the most potent non-optical measurement technique to observe turbulent flows. As this thesis heavily relies on the use of specialized hot-wires, their basic working principles shall be presented in this section.

\subsubsection{Working Principle}

A hot-wire is a thin, cylindrical metallic wire of a length $l$ of between usually $0.5 \mathrm{~mm}$ and $2 \mathrm{~mm}$ and of a diameter $d$ between $0.5 \mu \mathrm{m}$ and $5 \mu \mathrm{m}$ (Tropea et al.

\footnotetext{
${ }^{7}$ http://www.onera.fr/en/news/great-slma-wind-tunnel (as of 12.02.2015, 11:40)

${ }^{8}$ http: //Www.eswirp.eu/PDF/Press-Release-ESWIRP-completion.pdf (as of 12.02.2015, 12:20)
} 
2007, Jørgensen 2001). In most cases, the wire material is platinum or tungsten due to their steep, linear temperature dependence of resistivity (Comte-Bellot 1976). The wire is welded to prongs which ensure mechanical stability of the wire and connect the wire to the measurement electronics. A sketch of a hot-wire probe can be seen in figure 2.11 .

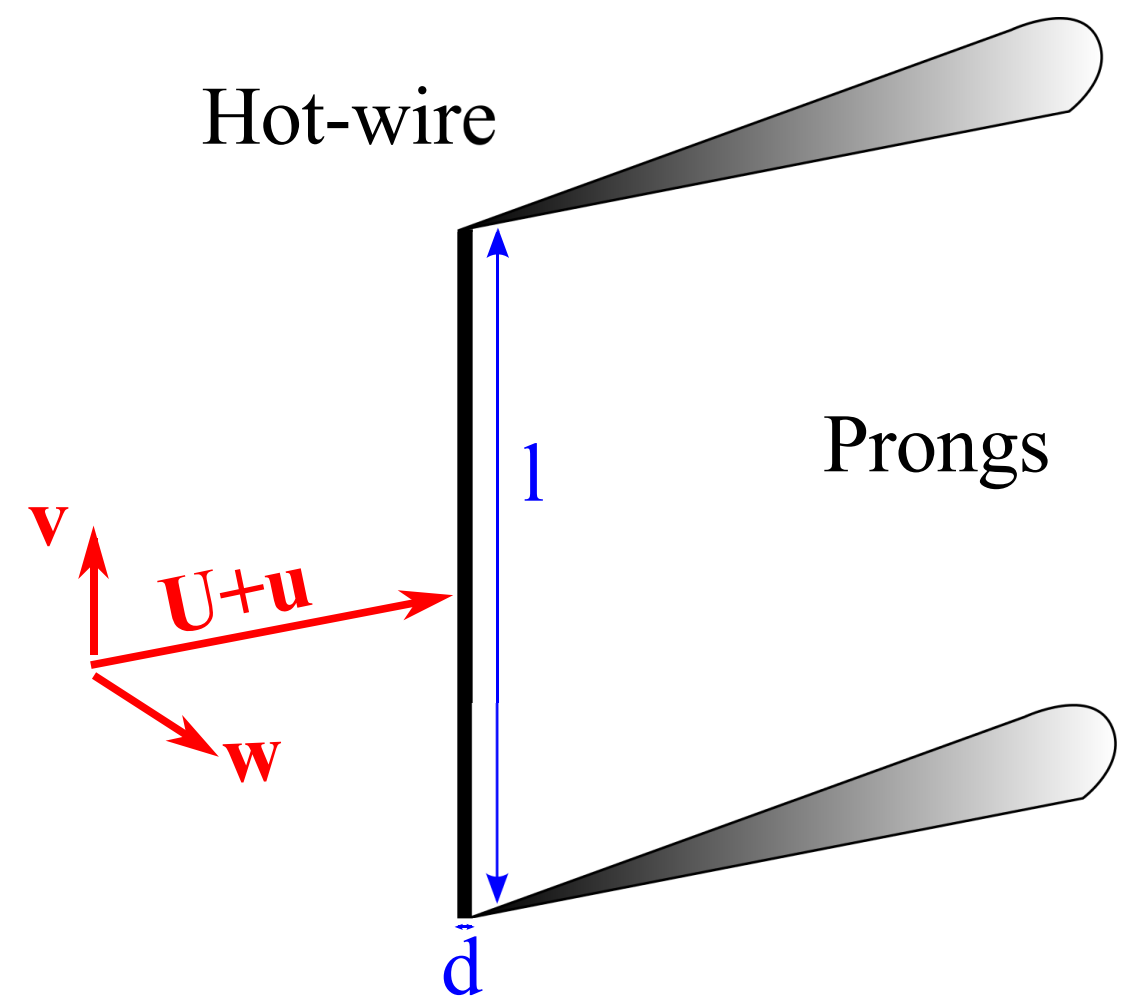

Figure 2.11: Sketch of a typical hot-wire

The wire is heated by the measurement electronics to a temperature significantly higher than the ambient temperature of the working gas. The fluid is advected with a mean speed $\mathbf{U}$ and small velocity fluctuations $\mathbf{u}$ (streamwise), $\mathbf{v}$ and $\mathbf{w}$ (transverse) over the heated wire, thereby cooling the wire. In a typical wind tunnel flow, the 
velocity fluctuations are much smaller than the mean speed and the streamwise velocity component $\mathbf{U}+\mathbf{u}$ dominates the contribution to the total heat loss from the transverse fluctuations $\mathbf{v}$ and $\mathbf{w}$. Thus, if placed normal to the mean flow, a hot-wire essentially reacts solely to the streamwise velocity component. One can write a heat balance equation for the wire-fluid system (Tropea et al. 2007), as follows:

$$
m_{w} c_{w} \frac{\mathrm{d} T_{w}}{\mathrm{~d} t}=R_{w} I_{w}^{2}-\left(T_{w}-T_{a}\right) \Phi
$$

Here, $m_{w} c_{w}$ is the thermal capacity of the wire, $T_{w}$ its temperature and $R_{w}$ its resistance. $I_{w}$ is the electrical current through the wire and, thus, $R_{w} I_{w}^{2}$ the heating rate. The cooling rate due to forced convection, $\Phi$, depends on the temperature difference of the wire and on the ambient temperature, $T_{a}$. The wire material is chosen to react linearly to temperature changes around the operating temperature. One can thus easily relate the wire resistance to its temperature as

$$
\begin{gathered}
R_{w}=R_{0}\left[1+\chi\left(T_{w}-T_{0}\right)\right] \\
R_{a}=R_{0}\left[1+\chi\left(T_{a}-T_{0}\right)\right]
\end{gathered}
$$

where $R_{a}$ is the resistance of the wire at ambient temperature, $T_{0}$ is a reference temperature usually chosen to be identical to the ambient temperature and $\chi$ is the temperature coefficient of resistivity. It is useful to define the overheat ratio, $a_{w}$, which is a measure of the temperature difference between the wire and the ambient 
fluid based upon the electronic properties of the wire:

$$
a_{w}=\frac{R_{w}-R_{a}}{R_{a}}
$$

Together with equations (2.2) and (2.3), one obtains a relation between the temperature and the resistance of the wire in the convenient form

$$
\frac{T_{w}-T_{a}}{T_{a}}=a_{w} \frac{1+\chi\left(T_{w}-T_{0}\right)}{\chi T_{a}} .
$$

\subsubsection{Constant Temperature Anemometer}

In order to actually measure turbulent velocities via the cooling rate $\Phi$, one can exploit the relation in equation (2.1) by designing an electrical circuit that keeps the resistance $R_{w}$ of the hot-wire and, thus, its temperature $T_{w}$, constant, yielding a simple relation to determine the heat loss:

$$
R_{w} I_{w}^{2}=\left(T_{w}-T_{a}\right) \Phi
$$

A change in the velocity directly leads to a change of the cooling rate $\Phi$ and thus to a measurable change in the heating current $I_{w}$. This can be achieved by placing the sensor in one arm of a Wheatstone bridge and using an operational 
feedback amplifier to feed the output current back to the bridge to restore the wire resistance. This is advantageous compared to a circuit in which the current is kept constant. While being significantly simpler to built, a Constant Current Anemometer is limited in frequency response by the thermal inertia of the wire. Using a Constant Temperature Anemometer (CTA) is, thus, preferable. A basic circuit of a Constant Temperature Anemometer is shown in figure 2.12.

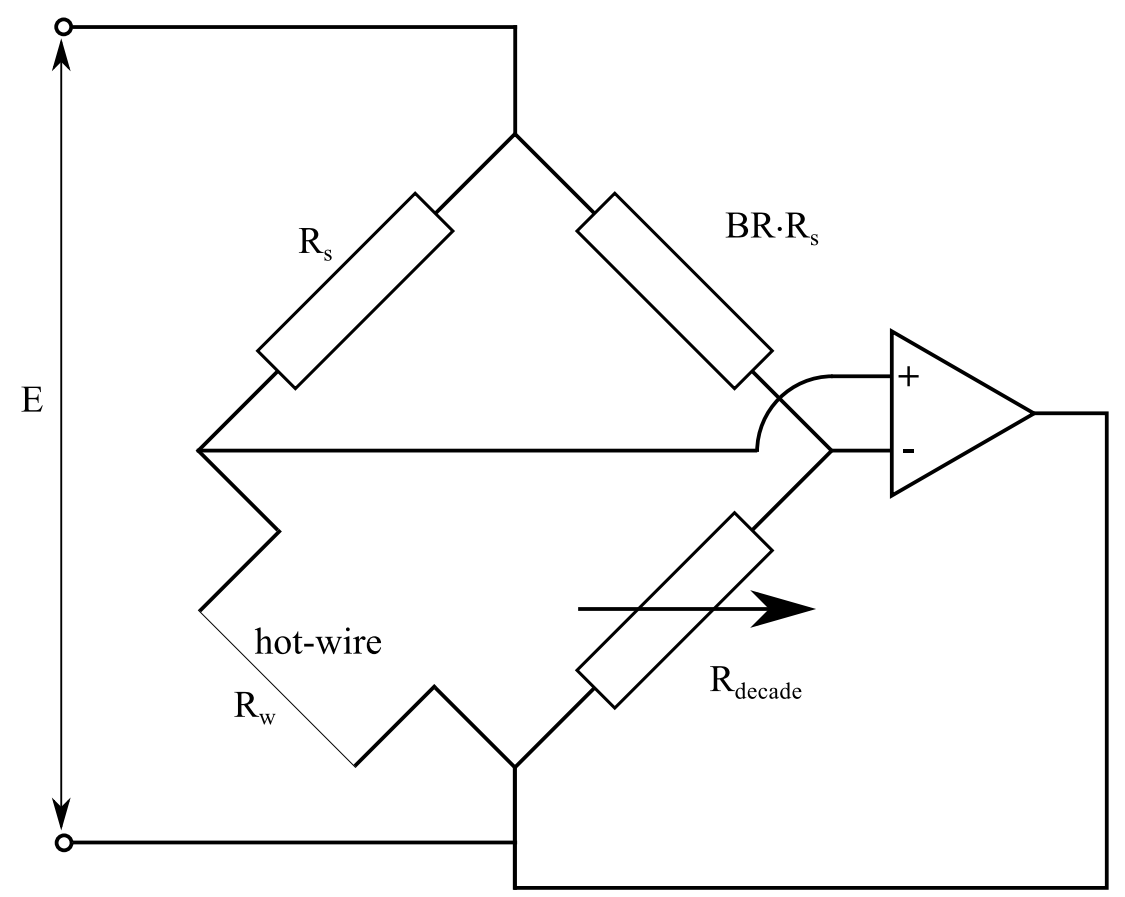

Figure 2.12: Sketch of a typical hot-wire circuit. This circuit transfers the cooling rate of the hot-wire into a measurable voltage signal $E$. The bridge ratio $B R$ of the resistances $R_{S}$ is typically 20 for standard hot-wires and 1 for NSTAPs. The resistance $R_{\text {decade }}$ has to be $R_{\text {decade }}=B R \cdot\left(1+a_{w}\right) \cdot R_{0}$ to balance the bridge.

As mentioned in subsection 2.1.4, a well-controlled ambient temperature is vital to conduct hot-wire measurements. This immediately becomes clear from equation (2.6). If the ambient temperature $T_{a}$ changes at the same time scales and magnitude 
as the cooling rate, due to the change of velocity, a CTA system is unable to distinguish the two effects. Figure 2.13 shows the temperature dependence of a CTA signal. There is a linear dependence of the CTA signal on the ambient temperature as expected via equation (2.6). With the temperature control capabilities of the VDTT (see subsection 2.1.4), the effect of temperature fluctuations on the velocity measurements can be neglected throughout. Even for fluctuations as large as $0.1 \mathrm{~K}$, which are much larger than those expected for the VDTT (see figure 2.7), the relative change of the response of the CTA system will be significantly smaller than $0.1 \%$.

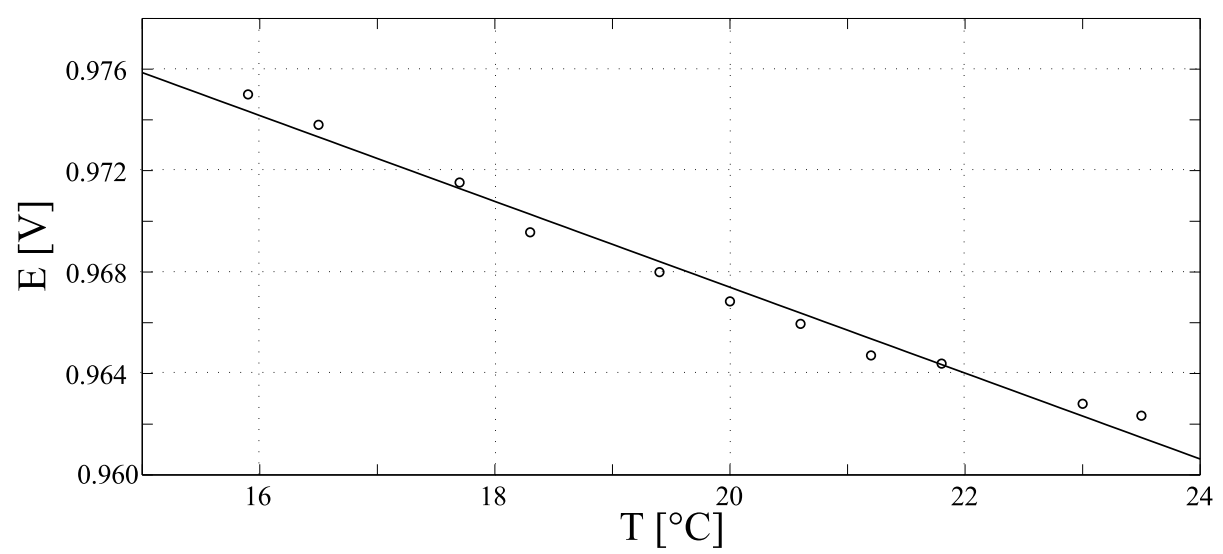

Figure 2.13: Typical temperature response for a NSTAP. The dependence of the voltage response from the CTA system on ambient temperature of the working gas is given. The data was taken at 8 bar with $\mathrm{SF}_{6}$ flowing at a constant mean speed. Black circles represent time averages over the turbulent signal measured by the NSTAP; the black line shows a linear fit to the data.

\subsubsection{The Nano Scale Thermal Anemometry Probes}

Considering that the size of the smallest eddies produced in the VDTT can be as small as $20 \mu \mathrm{m}$, it is clear that even the smallest of the commercially available 
hot-wires, having a length of about $500 \mu \mathrm{m}$, are insufficient for sophisticated measurements of smaller scales in the VDTT. The effect of eddies smaller than the sensor will be spatially averaged along the length of the wire so that no information about those structures can be gained. Therefore, nano scale thermal anemometry probes (NSTAPs) developed at the Department of Mechanical and Aerospace Engineering at Princeton University in the group of Alexander Smits (Bailey et al. 2010, Vallikivi et al. 2011) are used. These probes are significantly smaller than any other hot-wire available, with dimensions of either $100 \mathrm{~nm}$ by $2 \mu \mathrm{m}$ by $60 \mu \mathrm{m}$ or $100 \mathrm{~nm}$ by $2 \mu \mathrm{m}$ by $30 \mu \mathrm{m}$. In contrast to standard hot-wires, which are usually cylindrical wires produced in a Wollaston process (Wollaston 1813), the NSTAPs are produced from a silicon wafer, along the lines of standard semiconductor and microelectromechanical systems techniques. The resulting platinum probes are flat plates that are mounted onto standard hot-wire prongs to be compatible with the existing CTA equipment. Having a higher resistance (approximately $80 \Omega$ to $150 \Omega$ ) than classical hot-wires (about $5 \Omega$ ), several modifications to the CTA bridge are needed to operate the probes. The bridge ratio is limited to 1 for electrical stability of the feedback loop. The maximum possible overheat for NSTAPs of about 1.4 is significantly lower than that of standard hot-wires of about 1.8. Due to the high resistance of the NSTAPs, it is necessary to operate the CTA system with a temperature-stable external resistor. The resistor, along with its connecting cable, has to be carefully impedance-matched to the hot-wire and its connecting cable.

Extensive studies on the behavior of NSTAPs have been conducted by Bailey et al. (2010), Vallikivi et al. (2011), Hultmark et al. (2012), and Ashok et al. (2012), all of 
them confirming the validity of the wire response and concluding that the NSTAPs provide data comparable to classical hot-wires at large scales, while offering a significantly improved spatial and temporal resolution at small scales. Recent developments in the NSTAP technology include the production of crossed wires to extract two-dimensional flow information (Smits \& Hultmark 2014) and the design of nano-scale temperature probes (Arwatz et al. 2015).

\subsection{Experimental Setup and Datasets}

The turbulent velocity time series of this thesis are measured with the aforementioned hot-wires, located at the downstream end of the test section as discussed in section 2.1. The details of the experimental setup, the calibration procedure and the individual datasets are covered in the following section. The data in this thesis is based upon seven distinct datasets, containing several individual experiments each. The datasets named Decay,Decay_Modified and Decay_Near are used in the study of decaying turbulence in chapter 3. The datasets Statistic_Medium, Statistic_Large and Statistic_Huge are long time series, used to investigate the scaling behavior of turbulence in chapter 4, and the dataset S1MA is for the study of the small scale statistics of turbulence in chapter 5 .

\subsubsection{Experimental Setup}

The VDTT data of this thesis were obtained with two principal experimental configurations. In configuration A, hot-wire probes were placed on a two-dimensional 
transverse linear motion system installed to traverse the tunnel cross section $7.1 \mathrm{~m}$ behind the $180 \mathrm{~mm}$ mesh-size passive grid. The traverse system was utilized to investigate the quality of the flow regarding homogeneity. For the dataset Statistic_Medium, the probes were located around the centerline of the tunnel to ensure the best possible flow conditions. In configuration $\mathrm{B}$, from which the remaining datasets stem, the two-dimensional traverse was removed and an 8-meter long streamwise linear traverse on the tunnel floor was used to position the probes at arbitrary distances between $1.5 \mathrm{~m}$ and $8.3 \mathrm{~m}$ behind the grid. The traverse consisted of a threaded rod driven by a stepmotor that is controlled by the measurement computer outside of the tunnel. A picture of the probe configuration can be seen in figure 2.14 and a picture of the measurement section including the linear traverse in figure 2.15 .
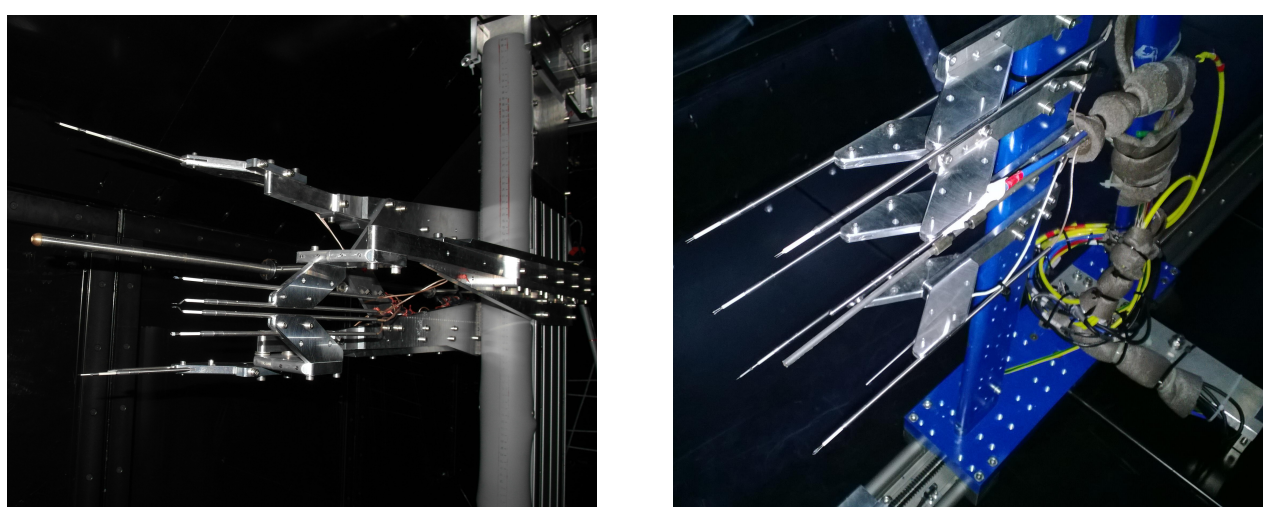

Figure 2.14: The left photograph shows probe configuration A, probes positioned on a two-dimensional traverse system. The right picture is a photograph of probe configuration $\mathrm{B}$, with the probes fixed in space in the transverse plane. 


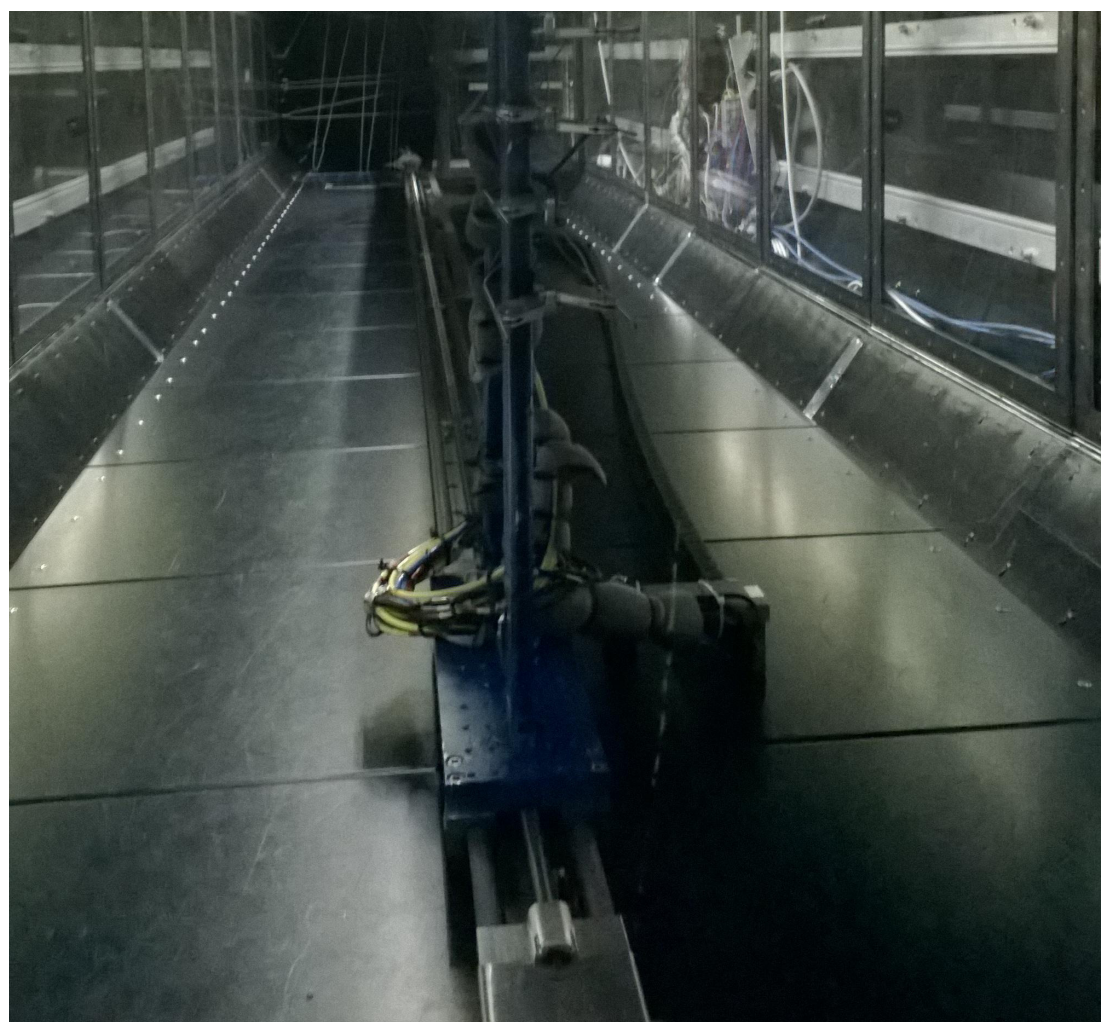

Figure 2.15: Downstream view on the probes and the linear traverse. The traverse is positioned at the nearest position to the grid.

Three types of probes were used to obtain the turbulent time series. The first two probe types were classical hot-wires produced by Dantec Dynamics ${ }^{9}$, one with a length of $1.25 \mathrm{~mm}$ and a wire diameter of $5 \mu \mathrm{m}$, dubbed from here on as 'P11' 10 . The second probe by Dantec Dynamics has a length of $450 \mu \mathrm{m}$ and a diameter of $2 \mu \mathrm{m}$ and is dubbed as 'Mini'. The major part of the data stems from NSTAPs with a length of $60 \mu \mathrm{m}$ and $30 \mu \mathrm{m}$, respectively, as discussed earlier. The probes

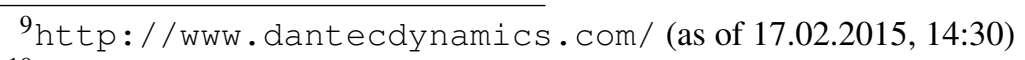

${ }^{10} \mathrm{https}$ ://www.dantecdynamics.com/miniature-wire-probe-straight (as of $17.02 .2015,14: 40)$
} 
are connected with 20 m of double-shielded RG223 BNC cables via feedthroughs in the wind tunnel walls to a Dantec Dynamics StreamLine 90N10 frame located outside of the VDTT. The frame is equipped with five CTA modules $90 \mathrm{C} 10$ and a temperature module to simultaneously gather data from up to five hot-wires. For the use of the NSTAPs with the Dantec CTA system, it is mandatory to use an external low noise resistor. The house-built "decade box" is adjusted individually to ensure an overheat ratio of 1.2 to 1.4 . The CTA modules are equipped with hardware signal conditioners including a hardware low-pass filter set to filter the signal at the Nyquist frequency. The data is digitized using a 12-bit National Instruments DAQCard-6062E ${ }^{11}$ and a 16-bit National Instruments NI-USB-6341 X Series Data card $^{12}$, respectively. The experiment is controlled via a LabView ${ }^{13}$ code originally designed by Florian Köhler and heavily modified by myself. Analysis of the data is done via a Matlab ${ }^{14}$ code originally written by Gregory P. Bewley and modified, extended and adjusted by myself.

\subsubsection{Calibration Procedure}

Hot-wires connected to a CTA circuit result in a voltage signal which is related to the velocity of the turbulent flow field. In general, the dependence of the voltage signal on the instantaneous velocity at the hot-wire is nonlinear. It can be shown (King 1914) that for relatively small velocity ranges, as in the VDTT, King's Law is

\footnotetext{
11 http://www.ni.com/pdf/manuals/370724c.pdf (as of 17.02.2015, 15:30)

12 http://sine.ni.com/nips/cds/view/p/lang/de/nid/209069 (as of 17.02.2015, 15:50)

${ }^{13}$ http: //www.ni.com/labview/ (as of 17.02.2015, 16:00)

${ }^{14}$ http: / / www . mathworks. com/ (as of 17.02.2015, 16:05)
} 
a sufficient approximation of the probe response, resulting in the relation

$$
E^{2}=a+b \cdot U^{n}
$$

Here, $E$ is the resulting voltage signal from the CTA output, $U$ the velocity and $a, b$ and $n$ are free parameters. The parameters in this equation are no universal constants but rather strongly depend on probe geometry, material, resistance, ambient and working temperature, overheat ratio and the conductivity of the working gas. In order to ensure the correct conversion of voltages into velocities, it is necessary to calibrate each individual probe prior to each experiment, using an independent measure of the velocity. This is done by the use of a pressure-based velocity measurement technique, using Pitot tubes (or rather by a combination of static and dynamic pressure tubes (Tropea et al. 2007)). Bernoulli's equation,

$$
\frac{\mathrm{d} p}{\rho}+U \mathrm{~d} U=0
$$

holds in a steady flow, where $p$ is the pressure, $\rho$ the density of the fluid and $U$ its velocity. Assuming an incompressible fluid with $\nabla \cdot \mathbf{u}$, separation of variables leads to

$$
p_{0}-p=\frac{1}{2} \rho U^{2}
$$


In this relation, $p_{0}$ is the pressure of the resting fluid (static pressure) and $p$ the pressure of the moving fluid (dynamic pressure). In the VDTT, the pressure difference $p_{0}-p$ is measured by static and dynamic pressure tubes that are connected to a pressure transducer located outside of the tunnel. With knowledge of the density of the working gas, it is possible to compute the flow velocity from this pressure difference as long as the turbulent fluctuations are small compared to the mean speed. By changing the speed of the fan, it is also possible to obtain the voltage response of the wires over a range of velocities to calibrate the probes with a fit to King's Law. A typical calibration curve at high pressure can be seen in figure 2.16.

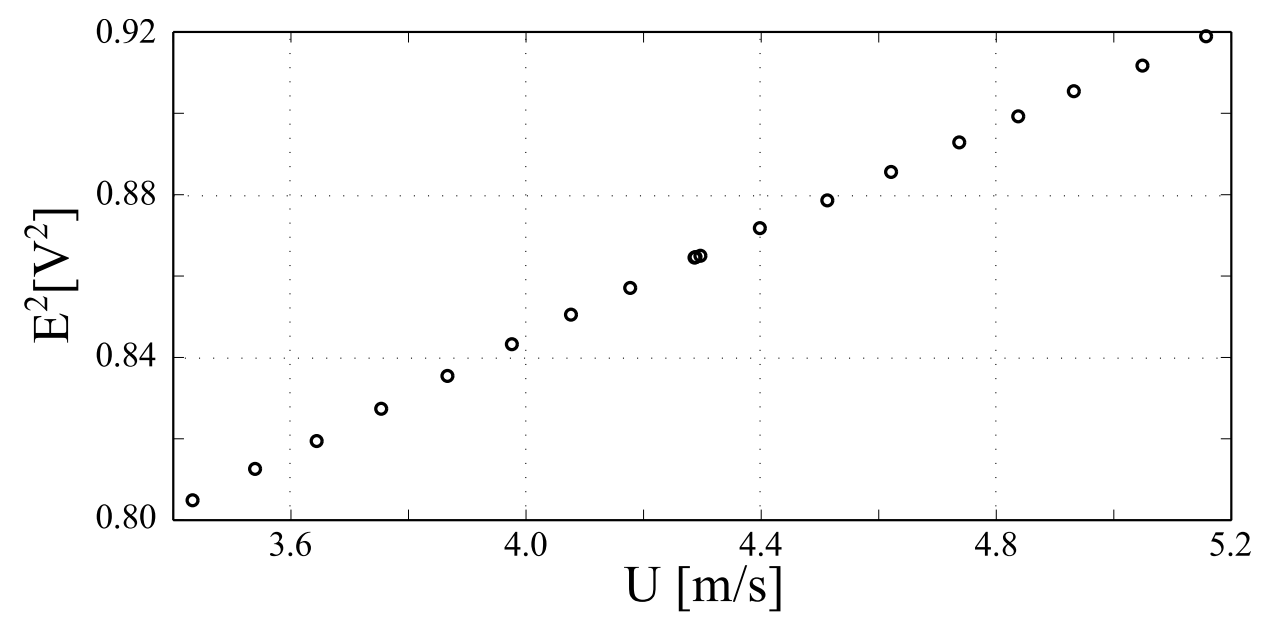

Figure 2.16: A typical calibration curve of a NSTAP at 13 bar $\mathrm{SF}_{6}$.

The calibration procedure is automated so that the measurement computer controls the tunnel fan along with the data acquisition. The signal from the pressure transducer is digitized using the same data acquisition card as for hot wire measurements. Between each measurement point, there is a time delay to allow the temperature control of the tunnel to stabilize. 


\subsubsection{Datasets}

The data in this thesis relies on three major classes of datasets. The results on the decay of turbulence are based upon the datasets Decay,Decay_Modified and Decay_Near. The largest dataset of these, the dataset Decay, consists of 36 individual experiments at different pressures, between air at 0.5 bar with a $12 \mathrm{~Hz}$ tunnel fan frequency and $\mathrm{SF}_{6}$ at 15 bar with a $20 \mathrm{~Hz}$ fan frequency, spanning mesh size Reynolds number $\operatorname{Re}_{M}$ between $10^{4}$ and $5 \cdot 10^{6}$. For each Reynolds number, turbulence statistics were acquired using NSTAPs, P11 and Mini probes at 50 logarithmically spaced distances between $1.5 \mathrm{~m}$ and $8.3 \mathrm{~m}$ from the grid. At each position, 5 minutes of data, or $1.8 \cdot 10^{7}$ samples, were measured.

\begin{tabular}{c|c} 
Dataset & Decay \\
\hline Probes & P11, Mini, NSTAP \\
Distance from the grid $[\mathrm{m}]$ & 1.5 to 8.3 \\
Sampling rate $[\mathrm{kHz}]$ & 60 \\
\# of positions & 50 \\
\# samples per position & $1.8 \cdot 10^{7}$ \\
Working gas & Air, $\mathrm{SF}_{6}$ \\
$p[\mathrm{bar}]$ & 0.5 to 15 \\
$v\left[\mathrm{~m}^{2} / \mathrm{s}^{2}\right]$ & $1.4 \cdot 10^{-7}$ to $3.3 \cdot 10^{-5}$ \\
\hline$U[\mathrm{~m} / \mathrm{s}]$ & 2.45 to 4.95 \\
$u^{\prime} / U$ & $1.58 \%$ to $3.56 \%$ \\
$\varepsilon\left[\mathrm{m}^{2} / \mathrm{s}^{3}\right]$ & $3.45 \cdot 10^{-3}$ to $1.77 \cdot 10^{-2}$ \\
$R_{\lambda}$ & 21 to 1450 \\
$\eta[\mu \mathrm{m}]$ & 21 to 1600 \\
$\lambda[\mathrm{mm}]$ & 1.57 to 20.3 \\
$L[\mathrm{~mm}]$ & 115 to 254
\end{tabular}

Table 2.3: Conditions of the decay measurements for the dataset Decay. The magnitude of the derived quantities changes with distance from the grid. Given are the quantities at the largest distance from the grid, obtained with NSTAPs. 
The three probes were located around the centerline of the tunnel and positioned by the streamwise linear traverse. For each measurement, the passive $180 \mathrm{~mm}$ grid was installed to produce turbulence. For an overview of the experimental conditions, see table 2.3.

The grid was modified for the dataset Decay_Modified in the following ways. In one iteration, steel wires with a diameter of $5 \mathrm{~mm}$ were wrapped along the transverse faces of the three central grid bar segments to locally change the detachment of turbulent wakes from the grid bars. For this modification, 6 of the above experiments haven been conducted between air at 1 bar and $\mathrm{SF}_{6}$ at 15 bar. One additional experiment at air at 1 bar was done using a grid modification with half-circles $40 \mathrm{~mm}$ in diameter placed along the streamwise direction of the grid bars. Photographs of the modifications can be seen in figure 2.17.
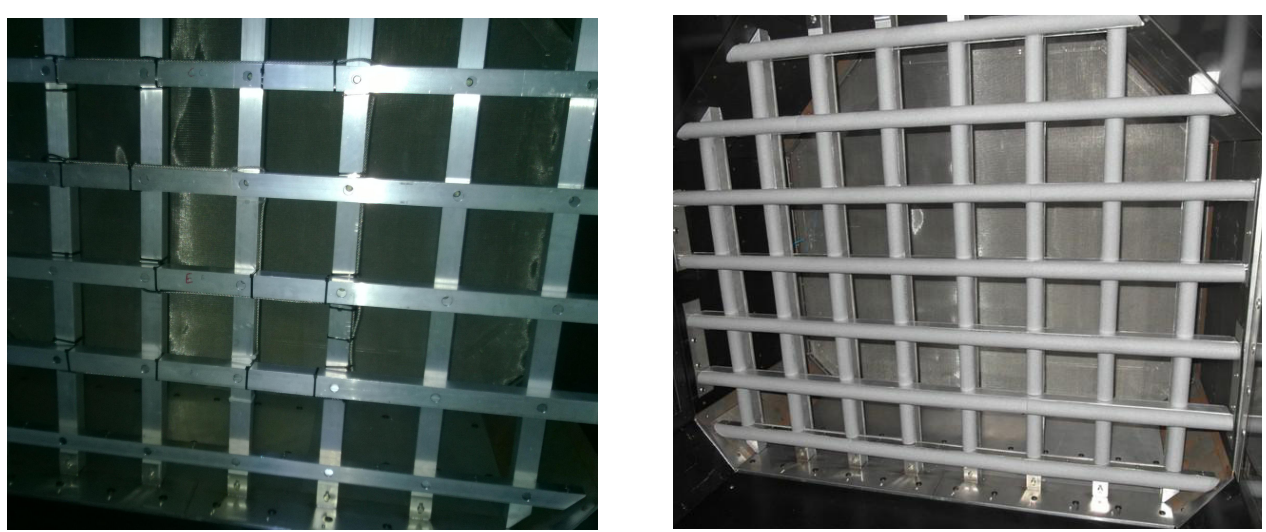

Figure 2.17: The left photograph shows the modification of the central grid gaps via steel wires, the right photograph the rounded grid. 
For the dataset Decay_Near, the probe setup was changed such that the minimum distance to the grid was altered, using a forward extension of the traverse wagon. The measurements were conducted in air at 1 bar at distances between $34 \mathrm{~mm}$ and $1.86 \mathrm{~m}$ behind the grid, using two P11 probes at 14 different distances from the grid. The experimental conditions for the datasets Decay_Modified and Decay_Near are summarized in table 2.4 .

\begin{tabular}{c|c|c} 
Dataset & Decay_Modified & Decay_Near \\
\hline Probes & P11, Mini, NSTAP & P11 \\
Grid distance $[\mathrm{m}]$ & 1.5 to 8.3 & 0.034 to 1.86 \\
Sampling rate $[\mathrm{kHz}]$ & 60 & 60 \\
\# positions & 50 & 14 \\
\# samples per position & $1.8 \cdot 10^{7}$ & $1.8 \cdot 10^{7}$ \\
Working gas & Air, $\mathrm{SF}_{6}$ & Air \\
$p[\mathrm{bar}]$ & 1 to 15 & 1 \\
$v\left[\mathrm{~m}^{2} / \mathrm{s}^{2}\right]$ & $1.4 \cdot 10^{7}$ to $1.55 \cdot 10^{-5}$ & $1.55 \cdot 10^{-5}$ \\
\hline$U[\mathrm{~m} / \mathrm{s}]$ & 4.2 to 4.3 & 3.9 \\
$u^{\prime} / U$ & $1.0 \%$ to $3.4 \%$ & $4 \%$ \\
$\varepsilon\left[\mathrm{m}^{2} / \mathrm{s}^{3}\right]$ & 0.002 to 0.02 & 0.1 \\
$R_{\lambda}$ & 70 to 1500 & 80 \\
$\eta[\mu \mathrm{m}]$ & 20 to 1250 & 430 \\
$\lambda[\mathrm{mm}]$ & 1.5 to 20 & 7.4 \\
$L[\mathrm{~mm}]$ & 124 to 138 & 70
\end{tabular}

Table 2.4: Conditions of the decay measurements for the datasets Decay_Modified and Decay_Near. Given are the quantities at the largest distance from the grid.

The data from the dataset Statistic_Medium is a collection of NSTAP data acquired with the $106.6 \mathrm{~mm}$ and the $180 \mathrm{~mm}$ grids, $7.1 \mathrm{~m}$ behind the grid. The 42 individual datasets are between 5 and 28 minutes long, measured at a constant positions along the transverse direction of the tunnel in configuration A (see subsection 2.4). An summary of the experimental conditions is given in table 2.5. 


\begin{tabular}{c|c} 
Dataset & Statistic_Medium \\
\hline Probes & NSTAP \\
Grid distance $[\mathrm{m}]$ & 7.1 \\
Sampling rate $[\mathrm{kHz}]$ & 60 and 200 \\
\# samples & $1.8 \cdot 10^{7}$ to $2.0 \cdot 10^{8}$ \\
Working gas & Air, $\mathrm{SF}_{6}$ \\
$p[\mathrm{bar}]$ & 1 to 15 \\
$v\left[\mathrm{~m}^{2} / \mathrm{s}^{2}\right]$ & $1.4 \cdot 10^{-7}$ to $1.5 \cdot 10^{-5}$ \\
\hline$U[\mathrm{~m} / \mathrm{s}]$ & 1.32 to 4.31 \\
$u^{\prime} / U$ & $1.99 \%$ to $4.38 \%$ \\
$\varepsilon\left[\mathrm{m}^{2} / \mathrm{s}^{3}\right]$ & 0.0003 to 0.035 \\
$R_{\lambda}$ & 50 to 1600 \\
$\eta[\mu \mathrm{m}]$ & 17 to 1080 \\
$\lambda[\mathrm{mm}]$ & 1.44 to 18.7 \\
$L[\mathrm{~mm}]$ & 72.7 to 150
\end{tabular}

Table 2.5: Experimental conditions of the dataset Statistic_Medium

The datasets Statistic_Large and Statistic_Huge were gathered in conjunction with the experiments corresponding to the dataset Decay, with the linear traverse being in the far downstream position $8.3 \mathrm{~m}$ behind the grid (with the exception of

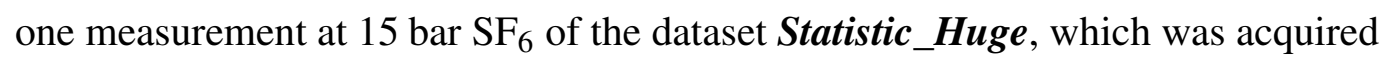
$6.9 \mathrm{~m}$ behind the grid). Statistic_Large consists of 14 measurements of the turbulent velocity between 6 and 9 hours long, whereas Statistic_Huge consists of 4 measurements between 2 and 3 days long. The datasets consist of simultaneous measurements with a P11, a Mini and an NSTAP. The experimental conditions of these datasets can be found in table 2.6. 


\begin{tabular}{c|c|c} 
Dataset & Statistic_Large & Statistic_Huge \\
\hline Probes & P11, Mini, NSTAP & P11, Mini, NSTAP \\
Grid distance [m] & 8.3 & 6.9 and 8.3 \\
Sampling rate $[\mathrm{kHz}]$ & 60 & 60 \\
\# samples & $1.3 \cdot 10^{9}$ to $1.9 \cdot 10^{9}$ & $1.0 \cdot 10^{10}$ to $1.5 \cdot 10^{10}$ \\
Working gas & Air, $\mathrm{SF}_{6}$ & $\mathrm{SF}_{6}$ \\
$p[\mathrm{bar}]$ & 1 to 14.5 & 1 to 15 \\
$v\left[\mathrm{~m}^{2} / \mathrm{s}^{2}\right]$ & $1.5 \cdot 10^{-7}$ to $1.55 \cdot 10^{-5}$ & $1.4 \cdot 10^{-7}$ to $2.6 \cdot 10^{-6}$ \\
\hline$U[\mathrm{~m} / \mathrm{s}]$ & 3.46 to 5.11 & 4.01 to 4.34 \\
$u^{\prime} / U$ & $1.57 \%$ to $3.45 \%$ & $2.7 \%$ to $3.6 \%$ \\
$\varepsilon\left[\mathrm{m}^{2} / \mathrm{s}^{3}\right]$ & 0.003 to 0.015 & 0.012 to 0.024 \\
$R_{\lambda}$ & 110 to 1450 & 300 to 1600 \\
$\eta[\mu \mathrm{m}]$ & 22 to 1030 & 19 to 191 \\
$\lambda[\mathrm{mm}]$ & 1.61 to 21.1 & 1.45 to 6.73 \\
$L[\mathrm{~mm}]$ & 103 to 138 & 126 to 129
\end{tabular}

Table 2.6: Experimental conditions of the datasets Statistic_Large and Statistic_Huge.

The data from the dataset S1MA was acquired in the S1MA wind tunnel in the scope of the ESWIRP project presented in section 2.2. The dataset S1MA consists of measurements with a $30 \mu \mathrm{m}$ NSTAP at 1 bar of air at mean flow speeds of $21 \mathrm{~m} / \mathrm{s}$ and $43 \mathrm{~m} / \mathrm{s}$, respectively. A summary of the flow parameters is given in table 2.7.

All tables with experimental conditions in this subsection are additionally given in Appendix A.

\subsubsection{Post-Processing}

The hot-wire voltage data is continuously written to the measurement computer hard disk in a 2 byte binary file format in individual files of 6 megabyte size. Every file thus contains $3 \cdot 10^{6}$ samples of the hot-wire voltage signal. In the post-processing, 


\begin{tabular}{c|c} 
Dataset & S1MA \\
\hline Probes & NSTAP \\
Sampling rate $[\mathrm{kHz}]$ & 200 \\
\# samples & $4.4 \cdot 10^{8}$ to $9.6 \cdot 10^{8}$ \\
Working gas & Air \\
$p[\mathrm{bar}]$ & 1 \\
$v\left[\mathrm{~m}^{2} / \mathrm{s}^{2}\right]$ & $1.5 \cdot 10^{-5}$ \\
\hline$U[\mathrm{~m} / \mathrm{s}]$ & 21 to 43 \\
$u^{\prime} / U$ & $1.2 \%$ to $1.7 \%$ \\
$\varepsilon\left[\mathrm{m}^{2} / \mathrm{s}^{3}\right]$ & 0.26 to 0.75 \\
$R_{\lambda}$ & 250 to 320 \\
$\eta[\mu \mathrm{m}]$ & 260 to 330 \\
$\lambda[\mathrm{mm}]$ & 9 to 10 \\
$L_{e}[\mathrm{~mm}]$ & 129 to 148
\end{tabular}

Table 2.7: Experimental conditions of the measurements conducted in the S1MA.

the voltage data is converted into velocities by applying King's Law (see equation (2.7)). The parameters for King's Law are obtained by a nonlinear least square fit (Seber \& Wild 2003) to the calibration curve using the built-in Matlab function nlinfit. CTA systems generally suffer from significant electric noise at high frequencies (Hutchins et al. 2012). To remove spurious high frequency signals, the velocity data is filtered with an $8^{\text {th }}$-order Butterworth filter. The filter frequency is chosen to cut the signal at the frequency at which the small scale part of the energy spectrum starts to grow in an unphysical way. The growth of the spectrum at very large scale can be attributed to the electrical noise stemming from the CTA system. An unfiltered energy spectrum is shown in figure 2.18. 


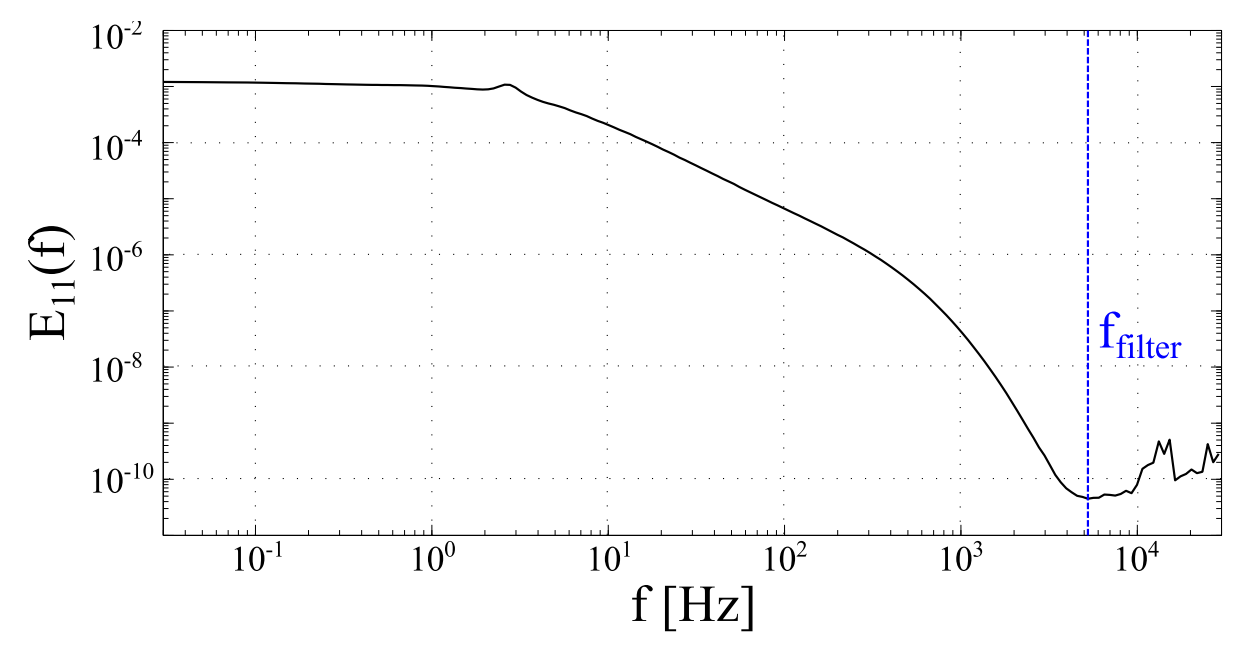

Figure 2.18: A one-dimensional energy spectrum from an unfiltered velocity signal from the dataset Statistic_Huge at 1 bar $\mathrm{SF}_{6}$. The cutoff frequency $f_{\text {filter }}$, depicted in blue, is chosen to be at the frequency at which electric noise starts to dominate the probe response.

In the VDTT, the cutoff frequency $f_{\text {filter }}$ can be estimated by the purely empirical formula

$$
f_{\text {filter }}=\frac{8}{\sqrt{v \cdot \frac{\mathrm{s}}{\mathrm{m}^{2}}}} e^{0.776-0.0938 \frac{\mathrm{d}}{\mathrm{m}}} \frac{1}{\mathrm{~s}}
$$

where $d$ is the distance from the grid and $v$ the kinematic viscosity of the working fluid. In case the automated estimation exceeds the Nyquist frequency, the cutoff frequency is chosen such that the filter range does not exceed the Nyquist frequency. 


\subsubsection{Computation of Flow Properties}

From the filtered velocity data, structure functions and spectra are computed via histograms for a finite number of separations. Unless otherwise stated, Kolmogorov's equation (1.24) is used to calculate the mean energy dissipation rate $\varepsilon$ from the structure functions, so that (neglecting the source term)

$$
\varepsilon=\max _{r}\left(-\frac{5}{4}\left(S_{3}(r)-6 v \frac{\mathrm{d}}{\mathrm{d} r} S_{2}(r)\right)\right)
$$

The derivative in this equation is approximated by the use of finite differences. The energy dissipation rate can then be used to calculate the Taylor length scale $\lambda$, the Taylor Reynolds number $R_{\lambda}$ and the Kolmogorov length scale $\eta$ in the isotropic approximation using

$$
\begin{aligned}
\lambda & =\sqrt{\frac{15 v}{\varepsilon}} u^{\prime}, \\
R_{\lambda} & =\frac{u^{\prime} \lambda}{v}, \\
\eta & =\left(v^{3} / \varepsilon\right)^{1 / 4} .
\end{aligned}
$$

The integral length scale $L$ is a quantity obtained from the velocity autocorrelation function (see subsection 1.2.2). As it is impossible to integrate over infinite times, and because noise prevents the autocorrelation to be identically zero at large scales, approximations must be employed. The common approximation uses exponential 
tail fitting to obtain the integral length scale. The autocorrelation function is continued by an exponential fit from a point where it crosses a threshold (chosen to be 0.05 , unless stated otherwise). The contribution of the large-scale part to the integral in equation (1.15) can then be calculated analytically from the fitted exponential decay. A second method for obtaining a large scale quantity closely related to the integral length scale is to define a length scale $L_{e}$ as the length scale where the autocorrelation function drops below $1 / e$ of its maximum value. This measure gives a quantity systematically smaller than the integral length scale, while preserving its scaling properties. 


\section{Decay of Turbulence}

I this chapter, I investigate how quickly a turbulent flow decays once it has been produced and which quantities govern its internal structure. In section 3.1, I address the fundamental question as to how fast turbulent kinetic energy decays after being produced by a passive grid. Section 3.2 considers a turbulence-intrinsic description of the decay. To generalize the results from the single passive grid decay studies, I conducted experiments with modified grid geometries whose results are covered in section 3.3. The chapter concludes with remarks on the measurement uncertainties in section 3.4. Parts of this chapter have been published in Physical Review Letters (Sinhuber, Bodenschatz \& Bewley 2015).

\subsection{The Decay of Turbulent Kinetic Energy}

As introduced in section 1.4, turbulence dissipates kinetic energy. This causes, in the absence of energy input into the flow, the turbulent motion to come to rest after a finite time. The answer to the seemingly simple question of how fast this process happens is not yet precisely known. It is neither known whether the theoretical 
predictions of Saffman (1967b) or Kolmogorov (1941c) hold true, nor, if they do, under what circumstances. There is no theoretical framework which accurately predicts the rate of decay for an arbitrary flow geometry. It is as a matter of fact even notoriously difficult to measure the rate of decay in experiments (Mohamed \& Larue 1990, Skrbek \& Stalp 2000, Hurst \& Vassilicos 2007, Krogstad \& Davidson 2010). The exact physics that control the decay are unknown, as is in particular whether or how the fundamental parameter of turbulent flows, the Reynolds number, affects the decay process. The original theoretical frameworks by Saffman and Kolmogorov do not include an explicit Reynolds-number dependence. In these theories, the rate at which turbulent kinetic energy decays should solely be governed by the large scales of the motion (Eyink \& Thomson 2000, Davidson 2011, Meldi et al. 2011). The line of thinking is that the initial structure at the time of production of the flow defines the decay rate (George 1992, Lavoie et al. 2007, Thormann \& Meneveau 2014). Numerous experimental studies agree with this physical picture (see figure 3.10), with many, but not all, of the results being along the lines of Saffman's prediction. These predictions break down once Reynolds numbers become very small (Ling \& Huang 1970, Perot \& de Bruyn Kops 2006), once the final period of decay is reached (Batchelor \& Townsend 1948b), or once side-wall effects start to dominate the flow (Stalp et al. 1999, Skrbek \& Stalp 2000). In Taylor-Couette flows (Huisman et al. 2012) and Rayleigh-Bénard convection (He et al. 2012), a transition to an asymptotic scaling behavior in extreme regimes of the flow has been observed, the so-called ultimate regimes. In the same spirit, for decaying turbulence, there are arguments for the existence of a self-similar decay at high 
Reynolds numbers, during which turbulence proceeds to remain self-similar to itself during the decay process, resulting in a constant Reynolds number during the decay combined with a power-law decay of energy with an exponent of -1 (Dryden 1973, Lin 1948, George 1992, Speziale \& Bernard 1992, Burattini et al. 2006, Lavoie et al. 2007, Kurian \& Fransson 2009). The theory is motivated by the fact that, a priori, neither Kolmogorov's nor Saffman's prediction can be proven to describe real flows. In fact, one can show that decaying turbulence can exhibit scaling quantities with the general form $u^{2} L^{m}$, with $m=5$ (Batchelor turbulence) or $m=3$ (Saffman turbulence). Using this to integrate equation (1.32),

$$
\frac{3}{2} \frac{\mathrm{d} u^{2}}{\mathrm{~d} t}=-C_{\varepsilon} \frac{u^{3}}{L}
$$

one obtains a general power-law decay of the form

$$
\frac{u^{2}}{U^{2}}=\left(1+\frac{m+2}{2 m} C \cdot t\right)^{-2 m /(m+2)}
$$

with an initial velocity $U$ and a constant $C$. Here, $u^{2}$ is a measure of the turbulent kinetic energy, $E$. It has been suggested that Saffman turbulence, corresponding to $m=3$, is a physical minimum of the decay exponent (Davidson 2009). The arguments supporting this claim, however, rely heavily on the applicability of the central limit theorem, which has to be reconsidered critically due to the loss of statistical independence from the non-locality of the Navier-Stokes equations. In 
the special case $m=2$, one obtains a self-similar decay of kinetic energy with

$$
\begin{aligned}
u^{2} & =C\left(t-t_{0}\right)^{-1}, \\
E(k) \underset{k \rightarrow 0}{\propto} k, & \\
R_{\lambda}(t) & =\text { const. }
\end{aligned}
$$

While Batchelor-type decay is connected to turbulence consisting of turbulent patches with significant conserved angular momentum and Saffman's theory to turbulence is governed by patches of non-negligible conserved linear momentum, self-similar turbulence would correspond to turbulence with conserved viscous contributions (George 1992). Especially, the results from the highest Reynolds number grid turbulence data available before the VDTT experiments by Kistler \& Vrebalovich (1966) and by Bewley et al. (2007) (in liquid helium) hint towards a decay exponent of -1 and thus self-similar decay. One must note, however, that the turbulence in the wind tunnel used by Kistler and Vrebalovich was of unusually high anisotropy. Furthermore, studies over a limited range of intermediate Reynolds numbers (George 1992, Speziale \& Bernard 1992, Burattini et al. 2006, Kurian \& Fransson 2009) show the tendency towards a slower decay with a possible limit of $m=2$, and thus $n=-1$. The experimental ability to adjust the Reynolds number in the VDTT independent of the outer flow geometry or mean speed facilitates the investigation into the possibility of an approach to self-similar decay in great detail. Especially, as the VDTT can reach Reynolds numbers significantly higher than 
those reported by Kistler \& Vrebalovich (1966), without the disadvantages of high anisotropy, and can cover a wider range of Reynolds numbers than in the studies of Kurian \& Fransson (2009).

\subsubsection{On the Power-Law Decay of Turbulence}

The most straightforward way to obtain information about the decay rate and the corresponding internal structure of turbulence is to investigate the decay of turbulent kinetic energy. As discussed in section 1.4, the decay of turbulent kinetic energy should follow a power-law of the form

$$
\frac{u^{2}}{U^{2}}=C\left(\left(t-t_{0}\right) \frac{U}{M}\right)^{n}
$$

Here, $U$ is the extrapolated mean velocity at the position of the passive grid, $t_{0}$ the virtual origin, $n$ the decay exponent and $C$ a prefactor. The offset of the power-law, the virtual origin, is the hypothetical position back in time where the energy would have been infinite, assuming a power-law decay for all times. To translate the spatial information of the data in the dataset Decay into temporal information, a simple Galilean transformation can be used to convert the distance from the grid $x / M$ in a time of decay $t$ by $t=x / U$ via the mean speed $U$. In total, as discussed in section 2.4.3, the dataset Decay contains 99 decay curves, spanning more than two orders of magnitude in mesh size Reynolds number, $10^{4}<\operatorname{Re}_{M}=U M / v<5 \cdot 10^{6}$, gathered with P11, Mini and NSTAP hot-wires. Each individual measurement consists of 
time series of the turbulent velocity at 50 logarithmically spaced distances from the grid. Figure 3.1 shows the decay curves from Mini probes at several Reynolds numbers.

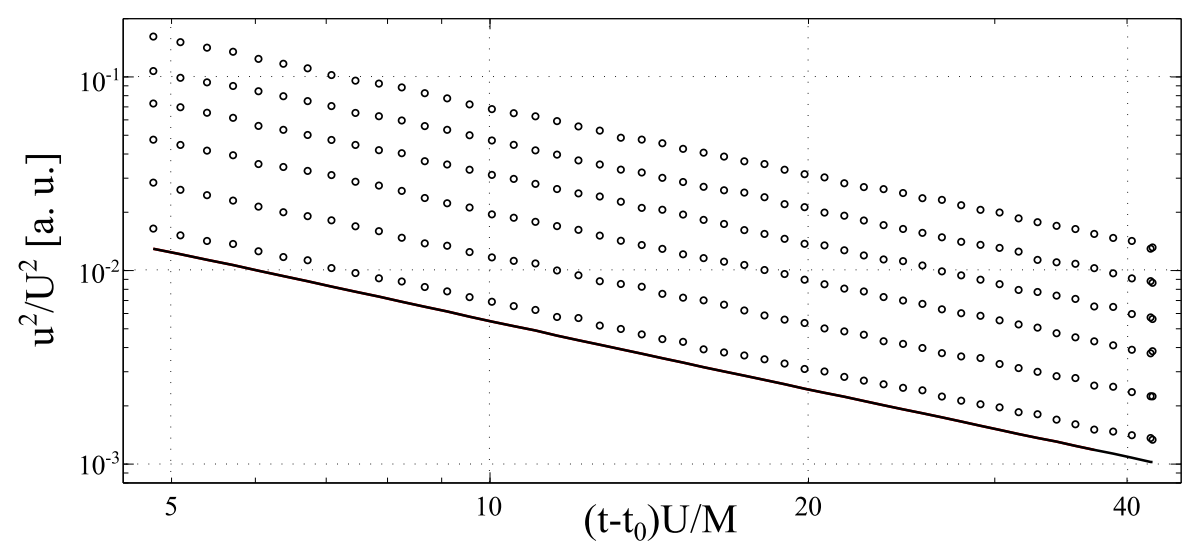

Figure 3.1: The decay of the normalized turbulent kinetic energy at six distinct Reynolds numbers measured by a Mini probe (o). The kinetic energy is normalized using the extrapolated mean speed at the grid position; the time is normalized by the mean speed over the mesh size. The offset $t_{0}$ of the abscissa is the virtual origin averaged over all 99 curves (see figure 3.2). The straight line is a global average over all Reynolds numbers and all probes. The Mini data is shifted incrementally for better visibility, with the bottom curve being unshifted. The Reynolds numbers of the curves are (from bottom to top) $29 \cdot 10^{3}, 41 \cdot 10^{3}, 83 \cdot 10^{3}, 17 \cdot 10^{4}, 20 \cdot 10^{4}$ and $48 \cdot 10^{4}$.

Despite spanning a wide range of Reynolds numbers, the individual curves are all consistent with the theorized power-law behavior in equation (3.6), as well as having virtually the same scaling over all experiments. The offset of time in figure 3.1 is the averaged virtual origin obtained from a three-parameter fit to the data. Figure 3.2 shows the virtual origin for all measurements of the dataset Decay. The resulting virtual origins show no trend with Reynolds number and scatter around the mean 
value of $t_{0} U / M=3.66$. The independence of Reynolds number is expected, as the virtual origin is presumably related to the geometry of the turbulence producing grid and the way wakes detach from the grid bars. Only different grid geometries should therefore lead to different virtual origins (Lavoie et al. 2007, Thormann \& Meneveau 2014, Comte-Bellot \& Corrsin 1966). Circular grid bars undergo the so-called drag crisis at Reynolds numbers of about $3 \cdot 10^{6}$ at which a drastic change of the drag coefficient occurs and the detachment behavior of wakes significantly changes (Schewe 1983). This feature is absent for rectangular grid bars (Schewe 2013), so that it is reasonable to assume that the virtual origin is independent of the Reynolds number.

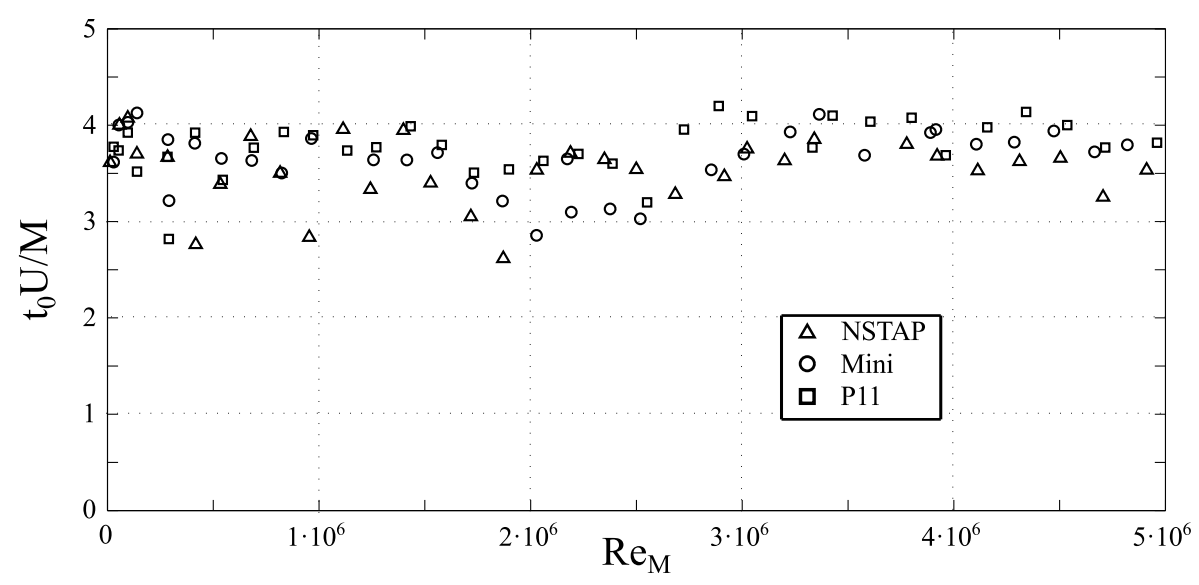

Figure 3.2: The resulting virtual origins from three-parameter fits to the decay curves in a semilogarithmic plot for P11 $(\square)$, Mini (o) and NSTAP $(\triangle)$ data. The data scatters around its mean value $t_{0} U / M=3.66$ with no apparent trend in Reynolds number.

The scatter in the virtual origin does however have a significant impact on the results directly derivable from a three-parameter fit to the data. As the virtual origin 
and the exponent in equation 3.6 are coupled quantities, their determination with a nonlinear least square algorithm would produce ambiguous results. Figure 3.3 shows the results for the decay exponents $n_{\text {free }}$ from a three-parameter fit as function of the Reynolds number.

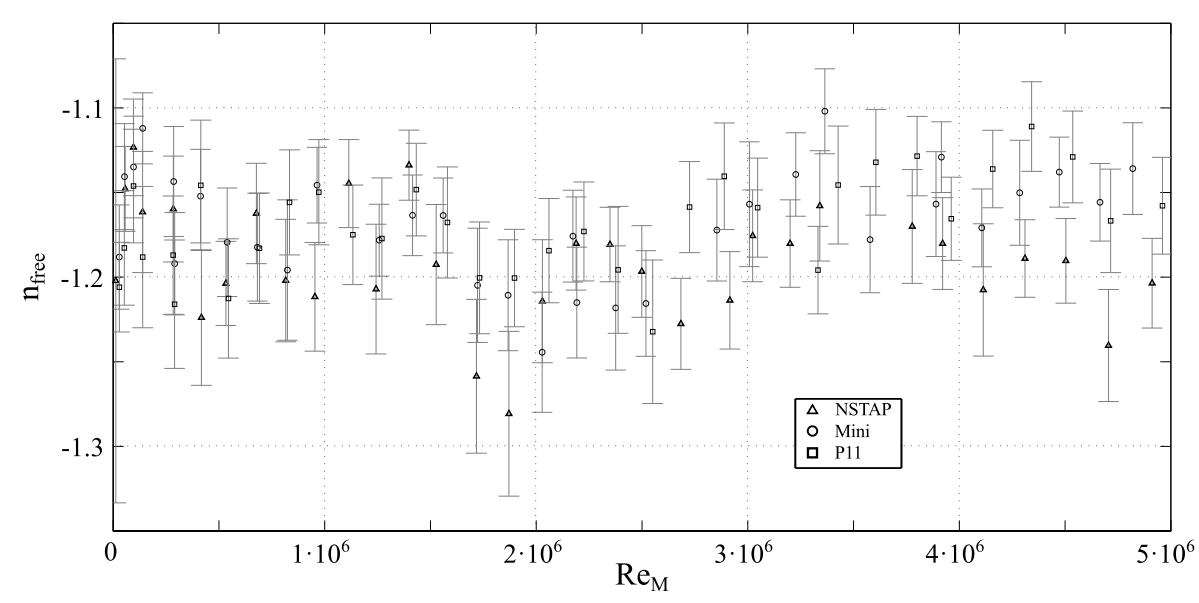

Figure 3.3: The resulting decay exponents from three-parameter fits to the decay curves. Due to the scatter in the virtual origin, the extracted decay exponents scatter significantly. The error bars correspond to the $95 \%$ confidence intervals.

This coupling of the decay exponent and the virtual origin be seen in figure 3.4. Although there is random scatter in the data, a clear dependence of the obtained decay exponent on the virtual origin and vice versa can be observed. The strong correlation between the decay exponent and the virtual origin prevents a precise analysis of a possible Reynolds-number dependence and further effects from a straightforward three-parameter fit. More sophisticated methods must be employed to extract meaningful physical information. 


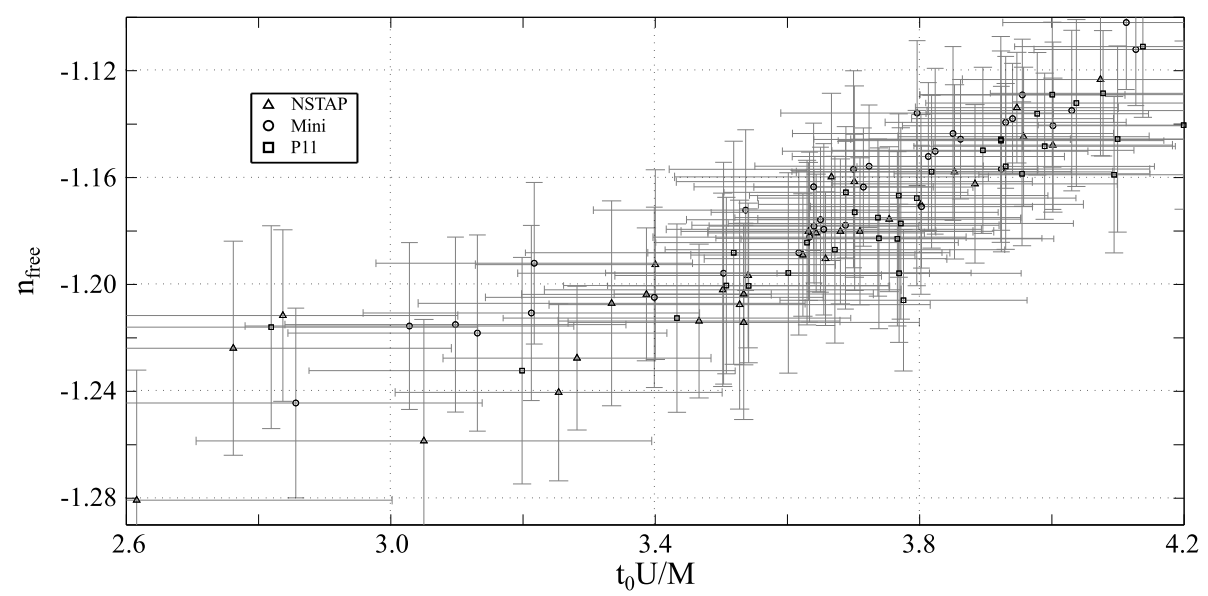

Figure 3.4: The dependence of the decay exponent on the virtual origin in a threeparameter Levenberg-Marquardt algorithm. Despite the scatter in the data, there is a distinct dependence of the exponent on the virtual origin. The error bars correspond to the $95 \%$ confidence intervals.

The physical meaning of the virtual origin lies in the near-field behavior of the flow directly behind the turbulence-producing grid. Figure 3.5 shows data from the Dataset Decay_Near, obtained with P11 probes as close as $3.4 \mathrm{~cm}$ downstream of the grid. The blue squares correspond to data obtained directly behind an open space of the grid, whereas the red data corresponds to data directly behind a grid bar. This leads to the behavior of the mean speed shown in the left figure. Initially, the mean speed behind an open space is much higher than behind a grid bar blocking the flow. Far away from the grid, however, the spreading of turbulent wakes leads to a homogeneous mean velocity profile across the cross-section of the tunnel. Analogous behavior can be found in the development of the kinetic energy, shown in the right plot in figure 3.5. Behind an open space, turbulent kinetic energy first builds up until some distance after the grid, due to the influence of growing wakes, 
while behind the grid bar, disturbances are initially high and decay directly. The global virtual origin depicted in these two figures as a black vertical line can be given a physical meaning in the following sense: The virtual origin for the classical grid used in these experiments is not directly related to the peak in the build-up of energy, but instead corresponds to the distance from the grid at which the differences in mean speed and turbulent kinetic energy between positions directly downstream of grid bars and open spaces vanish. The flow at this point thus has small spanwise shear contributing to the decay of energy. The virtual origin is therefore a measurable quantity, marking the beginning of freely decaying turbulence. Note that this applies to the classical grid of rectangular bars used in the datasets Decay and Decay_Near; other grids with greatly modified geometries like round grid bars, active grids or fractal grids might have a different relation between the virtual origin and the flow characteristics. This will have to be investigated in greater detail. Far downstream of the grid, the dependence of the flow characteristics on the near-field behavior vanishes (see figure 3.6). 

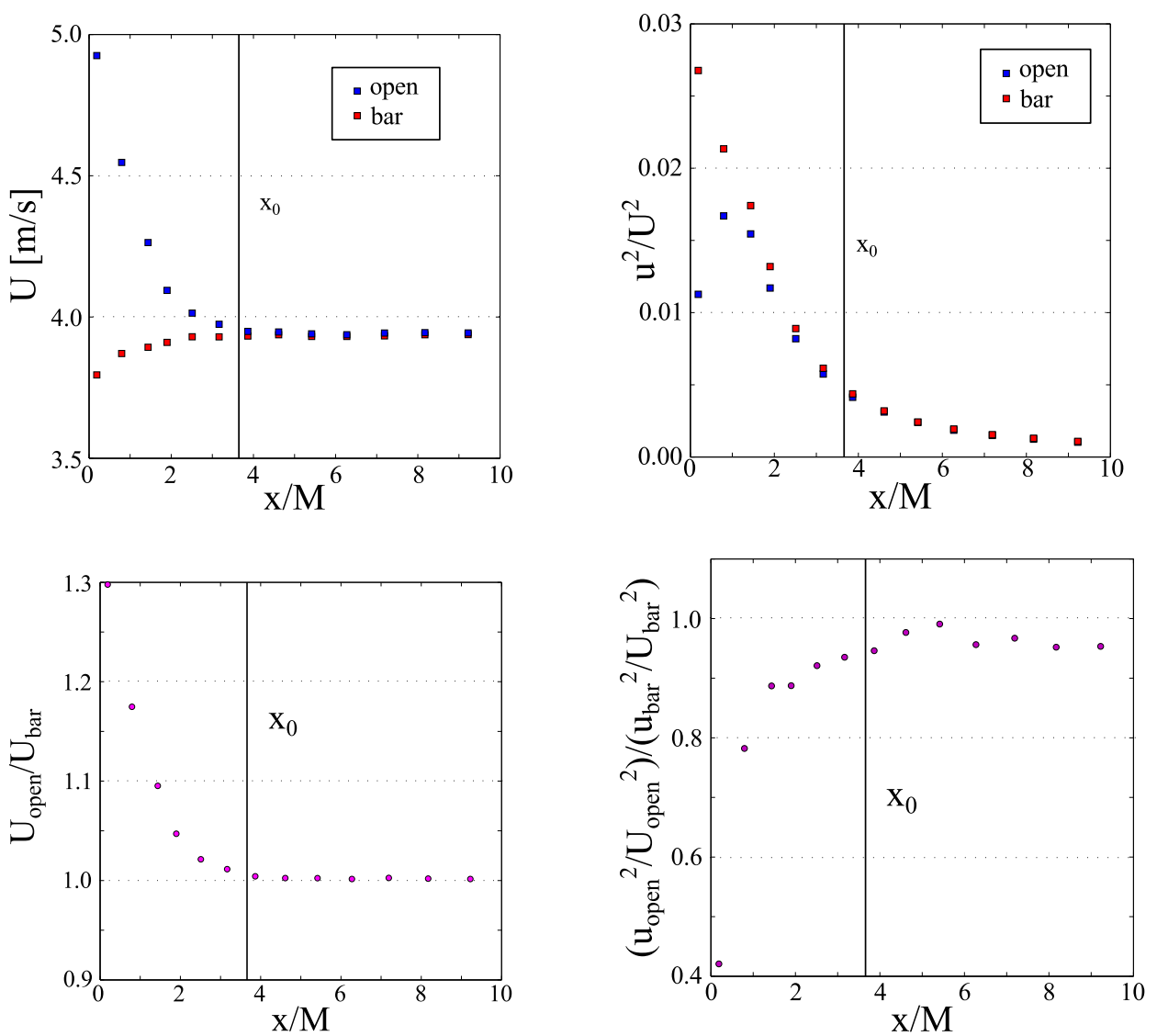

Figure 3.5: Decay measurements in the vicinity of the grid from the near-field dataset Decay_Near (see table 6.2). The top left figure shows the development of the mean speed directly downstream of a grid bar (red squares) and directly downstream of an open space (blue squares). The top right figure shows the decay of normalized turbulent kinetic energy for the same dataset. The bottom figure show the respective ratios. As expected, one observes a build-up of kinetic energy behind the open space due to turbulent mixing before the energy decays. The black vertical lines indicate the globally averaged virtual origin (see subsection 3.1.2) 

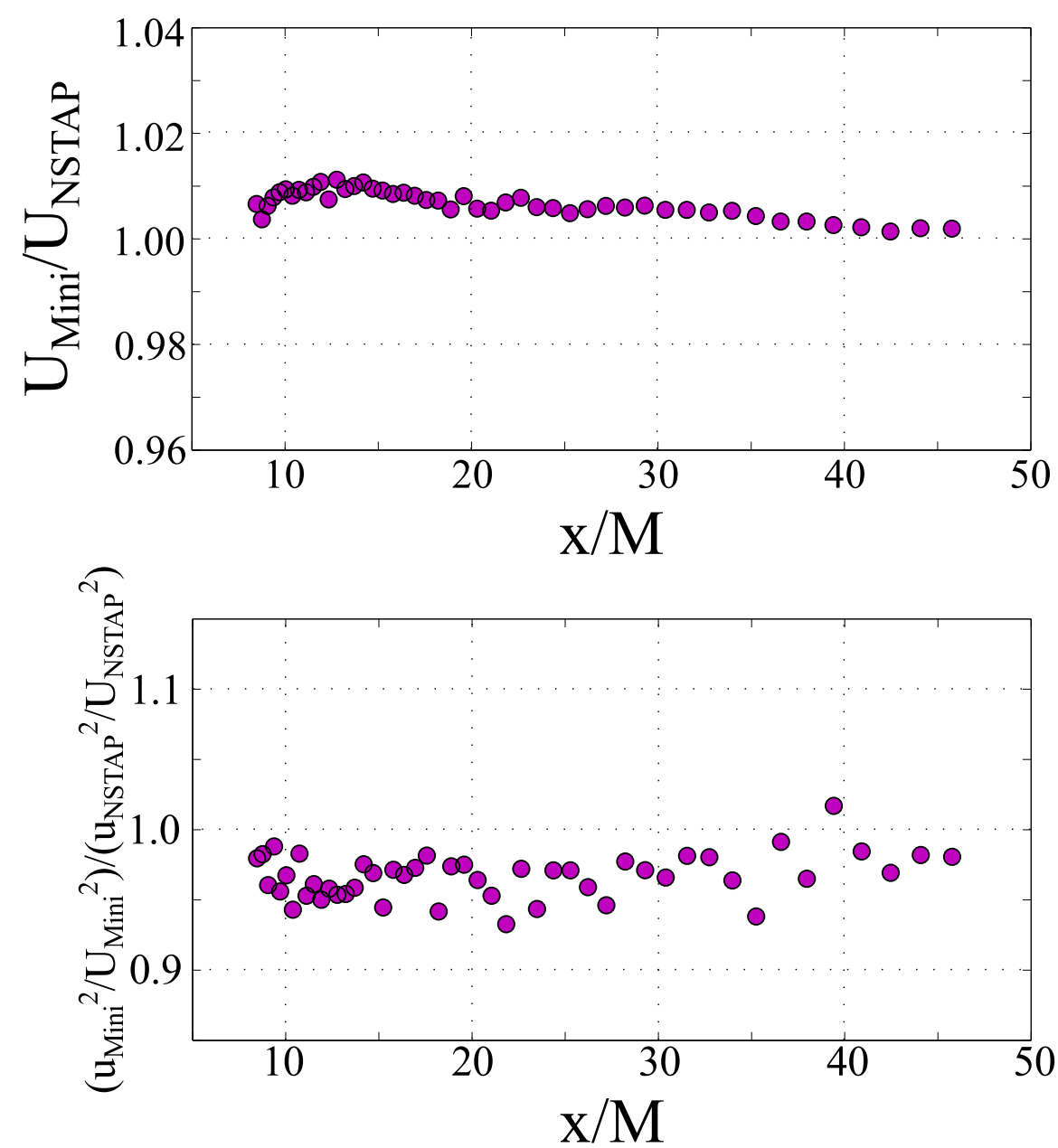

Figure 3.6: Decay measurements downstream of the grid from the dataset Decay (see table 6.1) at 2 bar $\mathrm{SF}_{6}$. The top figure shows the development of the ratio of the measured mean speeds obtained by a Mini probe and by an NSTAP at a different spanwise location. The bottom figure shows the ratio of the decay of normalized turbulent kinetic energy for the same experiment. Small deviations from unity can be attributed to slight uncertainties in the calibration procedure. 


\subsubsection{The Decay Exponent}

As the virtual origin does not depend on the Reynolds number and is connected to the tunnel geometry and that of the grid, both of which do not change between experiments, one way to improve the estimation of the decay exponent is to fix the virtual origin to its mean value. With this physically supported argument, the needed three-parameter fit of equation 3.6 to the data reduces to a two-parameter secondstep fit after the mean virtual origin has been determined. The resulting two fit parameters, the prefactor $C$ and the exponent $n$, are now significantly more reliable quantities than in the three-parameter fit. Figure 3.7 shows the resulting decay exponents. All individual resulting exponents are close to Saffman's prediction of $n=-1.2$, despite the Reynolds number spanning more than two orders of magnitude. The mean decay exponent of the accumulated data is slightly bigger, with $n=-1.18 \pm 0.02$. Neither the predictions for Batchelor turbulence nor those of a self-similar decay are compatible with the presented data. There is particularly no apparent trend towards a slower decay at high Reynolds numbers and no approach to a self-similar decay with an exponent of $n=-1$, contrary to earlier suggestions (see also figure 3.10 for a comprehensive comparison to earlier experimental findings). However, to be more precise, there is a negligible trend with Reynolds number. Fitting a line to the data of figure 3.7 in the semi-logarithmic coordinates shows an intersection with $n=-1$ at a Reynolds number of $R e_{M}=10^{50}$. For such a high Reynolds number, the largest scales of the flow needed to be of the size of a large galaxy with the smallest scales of the size of an atom. In other words, it categorically cannot be realized. Even though these findings cannot rule out a hypothetical sharp 
transition towards a self-similar decay at Reynolds numbers even higher than the experimentally observed Reynolds numbers of $R e_{M} \approx 5 \cdot 10^{6}$, none of the existing data supports such a transition.

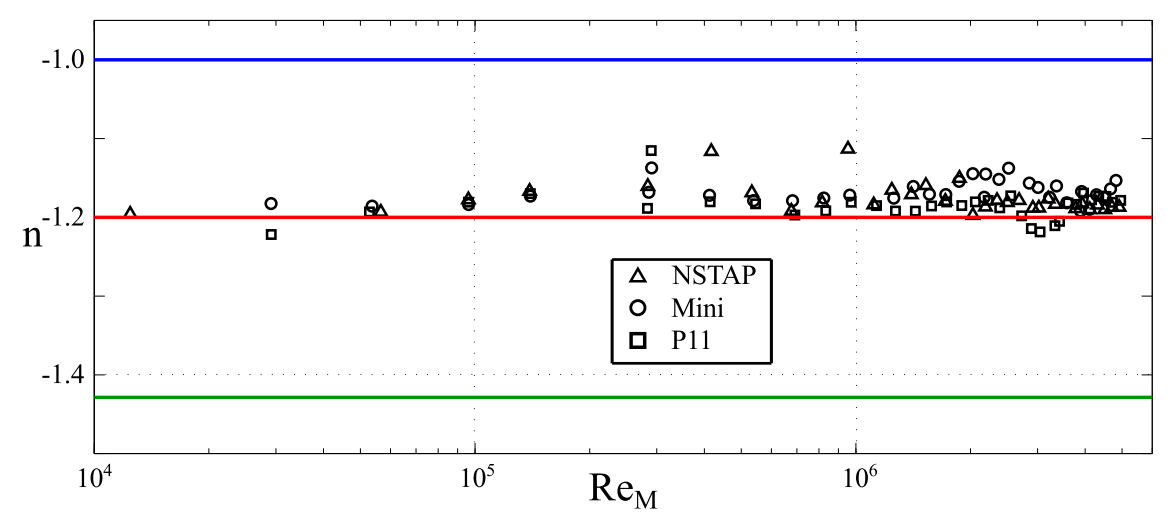

Figure 3.7: Resulting decay exponents from a two-parameter fit to the data with the virtual origin fixed to its mean value, $t_{0} U / M=3.66$. The horizontal lines represent the theoretical predictions for self-similar decay (blue), for Saffman turbulence (red) and for Kolmogorov turbulence (green). The mean value of the data is $n=-1.18$, with a standard deviation of 0.02, close to Saffman's prediction, with no significant trend in Reynolds number.

In order to study the constancy of the decay exponent with Reynolds number without needing to rely on the choice of a certain value of the virtual origin, a second method of analysis is applied to the data. For this, one assumes that the decay of turbulent kinetic energy $E_{i}\left(\operatorname{Re}_{i}, t\right)$ follows a general power-law at a certain Reynolds number $\mathrm{Re}_{i}$,

$$
E_{i}\left(\operatorname{Re}_{i}, t\right)=C_{i}\left(\operatorname{Re}_{i}\right)\left(t-t_{0}\right)^{n_{i}\left(\mathrm{Re}_{i}\right)},
$$


where $C_{i}\left(\mathrm{Re}_{i}\right)$ is a prefactor that might depend on the Reynolds number and $n_{i}\left(\mathrm{Re}_{i}\right)$ is the Reynolds number dependent decay exponent. The idea is that for fixed boundary conditions, and thus constant virtual origin $t_{0}$, one can relate the decay of energy $E_{i}\left(t, \operatorname{Re}_{i}\right)$ at a Reynolds number $\operatorname{Re}_{i}$ to the decay of energy $E_{j}\left(t, \operatorname{Re}_{j}\right)$ at a different Reynolds number $\operatorname{Re}_{j}$ in a formal way, such that

$$
E_{i}\left(\operatorname{Re}_{i}, t\right)=f\left(E_{j}\left(t, \operatorname{Re}_{j}\right), \operatorname{Re}_{i}, t\right) .
$$

Together with equation (3.7) at Reynolds numbers $\operatorname{Re}_{i}$ and $\operatorname{Re}_{j}$, one obtains

$$
E_{i}\left(\operatorname{Re}_{i}, t\right)=\frac{C_{i}\left(\operatorname{Re}_{i}\right)}{C_{j}\left(\operatorname{Re}_{j}\right)} E_{j}\left(\operatorname{Re}_{i}, t\right)^{\frac{n_{i}\left(\mathrm{Re}_{i}\right)}{n_{j}\left(\mathrm{Re}_{j}\right)}}
$$

By calculating the logarithmic derivative of this expression, a relative decay exponent $n_{r}\left(\operatorname{Re}_{i}, \operatorname{Re}_{j}\right)$ is obtained with

$$
n_{r}\left(\operatorname{Re}_{i}, \operatorname{Re}_{j}\right)=\frac{\partial \log E_{i}}{\partial \log E_{j}}=\frac{n_{i}\left(\operatorname{Re}_{i}\right)}{n_{j}\left(\operatorname{Re}_{j}\right)}
$$

With this technique, it is possible to extract relative information about the Reynolds-number dependence of the decay exponent without a priori knowledge about the virtual origin, therefore removing the ambiguity in the determination. Note that this technique does not demand that individual decay curves strictly follow 
power-laws; generalized power-laws with an arbitrary cutoff function $g(t)$ of the form $E_{i}\left(\operatorname{Re}_{i}, t\right)=C_{i}\left(\operatorname{Re}_{i}\right) g(t)\left(t-t_{0}\right)^{n_{i}\left(\mathrm{Re}_{i}\right)}$ produce the same results.

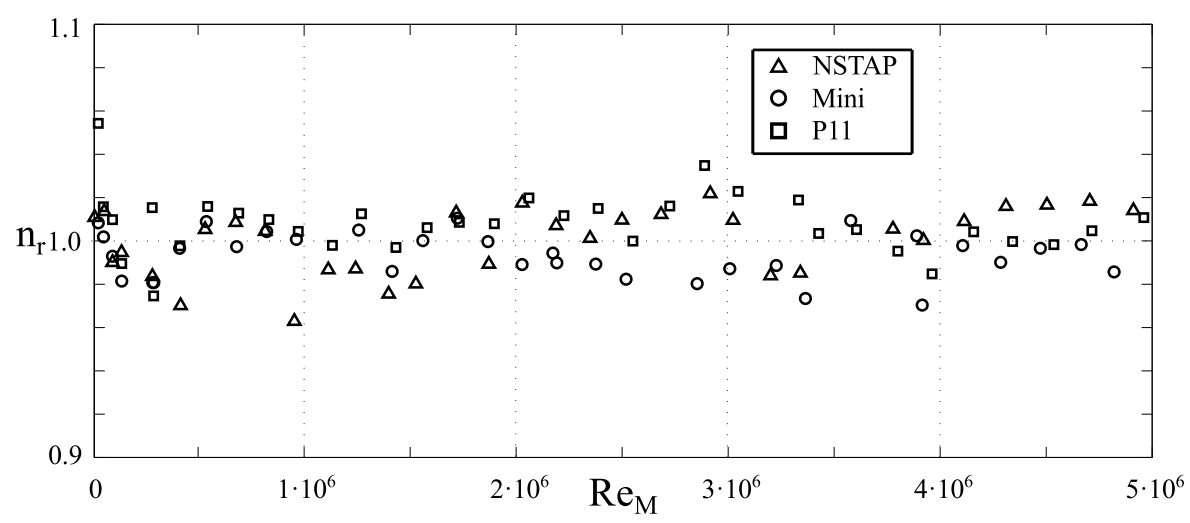

Figure 3.8: The relative decay exponents as in equation (3.10) in a linear plot. These relative exponents are invariant in the Reynolds number.

The relative decay exponents shown in figure 3.8 are computed choosing $E_{j}$ to be the averaged curve over all decay curves (see figure 3.1) and by using a finite difference formula for unequal sub-intervals (Singh \& Bhadauria 2009) to calculate the derivative in equation (3.10). The invariance in Reynolds number of the relative decay exponent independently confirms the findings of figure 3.7. Scattering around $n_{r}=1$, the relative decay exponent supports the argument that there is no general influence of the Reynolds number on the rate of decay. In the classical theories, the prefactor $C$ in the decay should be a constant of Reynolds number as well. Following Batchelor \& Townsend (1948a), the prefactor should solely depend on the grid geometry and its drag per unit area. Experimental studies at lower Reynolds numbers and for several fixed grid geometries, however, find that $C$ is indeed a decreasing function of Reynolds number for $\operatorname{Re}_{M}<10^{4}$ (Kurian \& Fransson 2009). 
The data from the dataset Decay in figure 3.9, however, shows a Reynolds number trend of an increasing $C$ until a possible saturation at high Reynolds numbers. This is consistent with the picture that the constant $C_{\varepsilon}$ in equation (1.32) only reaches a constant value for high Reynolds numbers.

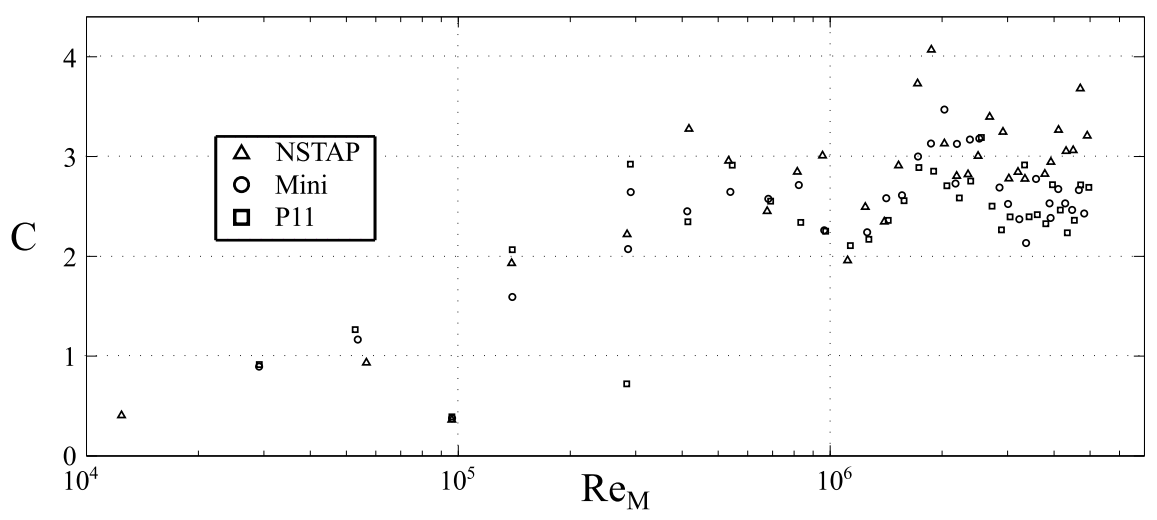

Figure 3.9: The prefactor of the decay of turbulent kinetic energy corresponding to the two-parameter fits and decay exponents in figure 3.7. At lower Reynolds numbers, a distinct trend towards larger prefactors can be observed which seems to saturate for $R e_{M} \gtrsim 10^{6}$.

As discussed earlier, previous experimental results implied that there might be an approach to self-similar decay at high Reynolds numbers. A dependence of the decay exponent on the Reynolds number has also been observed in numerical simulations (Burattini et al. 2006, Ishida et al. 2006, Perot 2011). Figure 3.10 shows the gathered experimental data previously obtained in combination with the VDTT results from figure 3.7 .

Judging only from the previous experimental data, which is subject to significant scatter, the assumptions of a trend towards a slower decay rate at higher Reynolds numbers is not unreasonable. Combined with the new results from dataset Decay, 


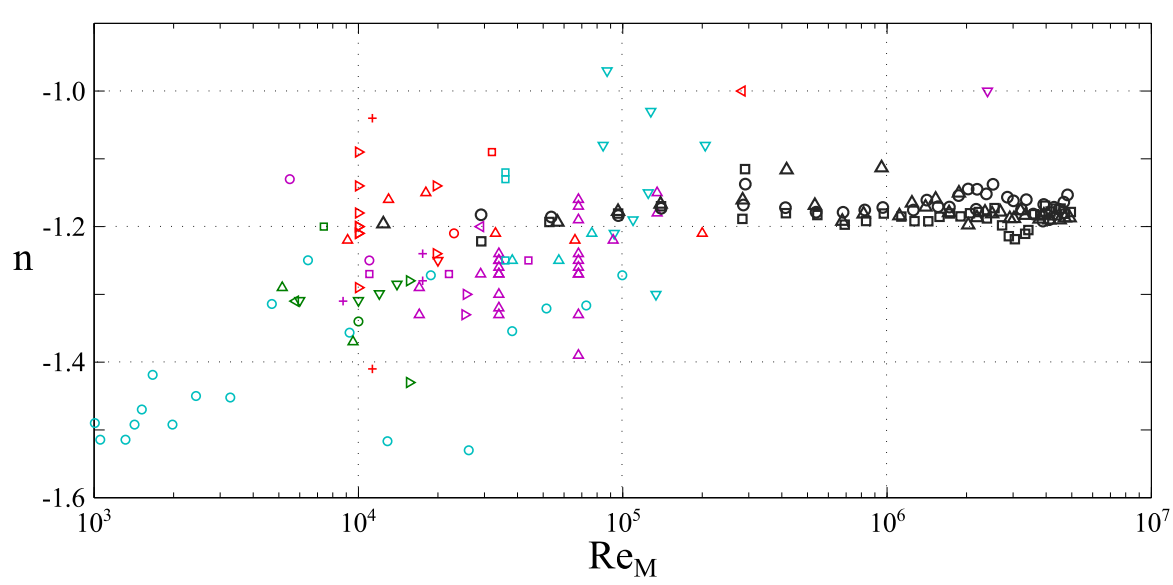

Figure 3.10: Comparison of the Reynolds number dependence of the decay exponents $n$ of the dataset Decay (black symbols, data as in figure 3.7) with published experimental data from previous experiment Batchelor \& Townsend (1948a) (o), Wyatt (1955) ( $\square$ ), Uberoi (1963) (+), Comte-Bellot \& Corrsin $(1966)(\triangle)$, Kistler \& Vrebalovich (1966) $(\nabla)$, Uberoi \& Wallis (1966) $(\triangleleft)$, Van Atta \& Chen (1968) ( $\triangleright$ ), Warhaft \& Lumley (1978) (॰), Sreenivasan et al. (1980) ( $\square$ ), Sirivat \& Warhaft (1983) $(\triangle)$, Mohamed \& Larue (1990) $(\nabla)$, Yoon \& Warhaft (1990) $(\triangleleft)$, Makita (1991) $(\triangleright)$, Mydlarski \& Warhaft (1996) (○), van Doorn et al. (1999) ( $\square$ ), Poorte \& A. (2002) (+), White et al. (2002) $(\triangle)$, Antonia et al. (2003) ( $\nabla)$, Bewley et al. (2007) ( $\triangleleft)$, Lavoie et al. (2007) $(\triangleright)$, Kurian \& Fransson (2009) (॰), Krogstad \& Davidson (2011) ( $\square$ ), Valente $\&$ Vassilicos (2011) $(\triangle)$ and Thormann \& Meneveau $(2014)(\nabla)$.

however, it becomes evident that the generic high Reynolds number limit is most probably not the self-similar decay. However, the data suggest a transition from Batchelor to Saffman turbulence at very low Reynolds numbers.

\subsection{Turbulence-Intrinsic Description}

So far, the study of the decay of turbulence was performed in a straightforward way by means of the time-dependence of a global quantity, the turbulent kinetic energy. 
While this approach already provides meaningful insight on the Reynolds-number dependence of the decay process, it lacks a direct connection to the underlying physics of the decay process. As described in section 1.4, the internal, large-scale structure of a turbulent flow is believed to determine its behavior when freely decaying. Conserved quantities are responsible for the persistence of turbulence. A description of the decay of turbulence based upon its intrinsic quantities, e.g., its length scales and dissipation rate, therefore seems to be a more natural approach to understanding the physics of turbulent decay and it shall be discussed in this section. Of further advantage is that the description based upon turbulence itself eliminates the need of determining a virtual origin completely, as the statistics of the instantaneous flow properties are real physical quantities that do not depend on an arbitrary time offset.

\subsubsection{The Large-Scale Flow Structure}

The classical theory by Kolmogorov (1941c) predicts the energy dissipation rate $\varepsilon=-(3 / 2) \mathrm{d} u / \mathrm{d} t=C_{\varepsilon} u^{3} / L$ to be constant with regards to time and Reynolds number. As long as the Reynolds number is high enough, $C_{\varepsilon}$ indeed approaches a constant value of order one in many flows (Sreenivasan 1998). As described in section 1.4, assuming a power-law relation between the integral length scale $L$ and the energy $u^{2}$, one is able to integrate the equation above to obtain the power-law decay of energy in the form $u^{2} \sim\left(t-t_{0}\right)^{n}$. By investigating the relation between $L$ and $u^{2}$ directly, it is possible to independently characterize the type of turbulence, as the classical theories demand $L \propto\left(u^{2}\right)^{k}$ with $k=-1 / 2$ for self-similar decay, 
$k=-1 / 3$ for Saffman type turbulence and $k=-1 / 5$ for Batchelor turbulence.

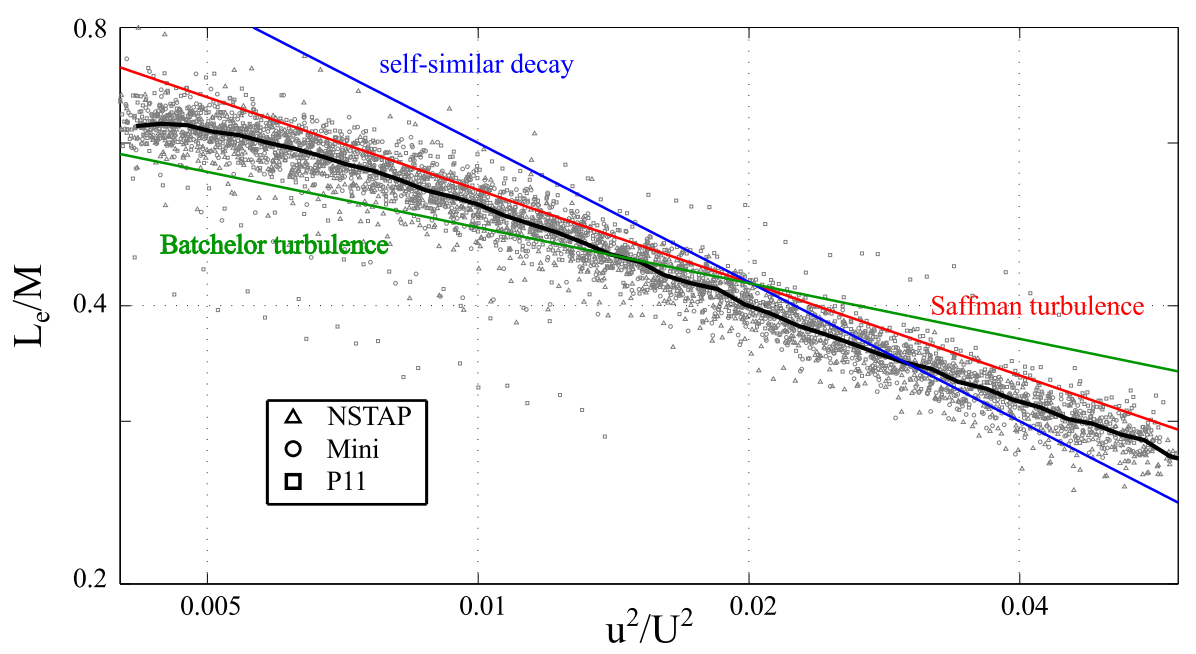

Figure 3.11: Logarithmic plot of the normalized integral length scale $L / M$ versus the normalized turbulent kinetic energy $u^{2} / U^{2}$. The gray point are calculated from the turbulent velocity signal at each position and each Reynolds number from the Dataset Decay without discrimination regarding any quantity. Here, $L_{e}$ is calculated as the length scale where the velocity autocorrelation drops to $1 / e$ of its maximum value. The black curve is the median of all curves, calculated in logarithmically spaced bins, the blue line is the corresponding prediction for a self-similar decay, the red line is that of Saffman's prediction and the green line is that of Kolmogorov's theory.

Figure 3.11 shows the resulting relation between the integral length scale based upon the scale at which the velocity autocorrelation function drops to $1 / e$ of its maximum value and the turbulent kinetic energy. Note that the lower right part of the figure corresponds to small times, i.e., short distances from the grid, and the upper left part to large times. The resulting black median curve over all experiments is compatible with the predictions for Saffman's turbulence. The deviations from a power-law behavior for large times can be attributed to the tunnel's side walls, 
affecting the flow at very large distances from the grid. Neither the predictions of self-similar decay nor those of Batchelor type turbulence are compatible with the data. A power-law fit to the data gives $L \propto\left(u^{2}\right)^{-0.35}$, which is close to Saffman's $L \propto\left(u^{2}\right)^{-1 / 3}$ and precisely reproduces a decay exponent of $n=-1.18$, as found in figure 3.7. There is no apparent approach towards a self-similar decay with high Reynolds numbers, which confirms the finding in figure 3.10. Note that the analysis presented here is completely independent of the one in section 3.1 and, explicitly, does not depend on the virtual origin. Figure 3.12 shows the individually fitted exponents $k$ in the relation $L \propto\left(u^{2}\right)^{k}$. The results are consistent with the findings from section 3.1.2, independently of the virtual origin.

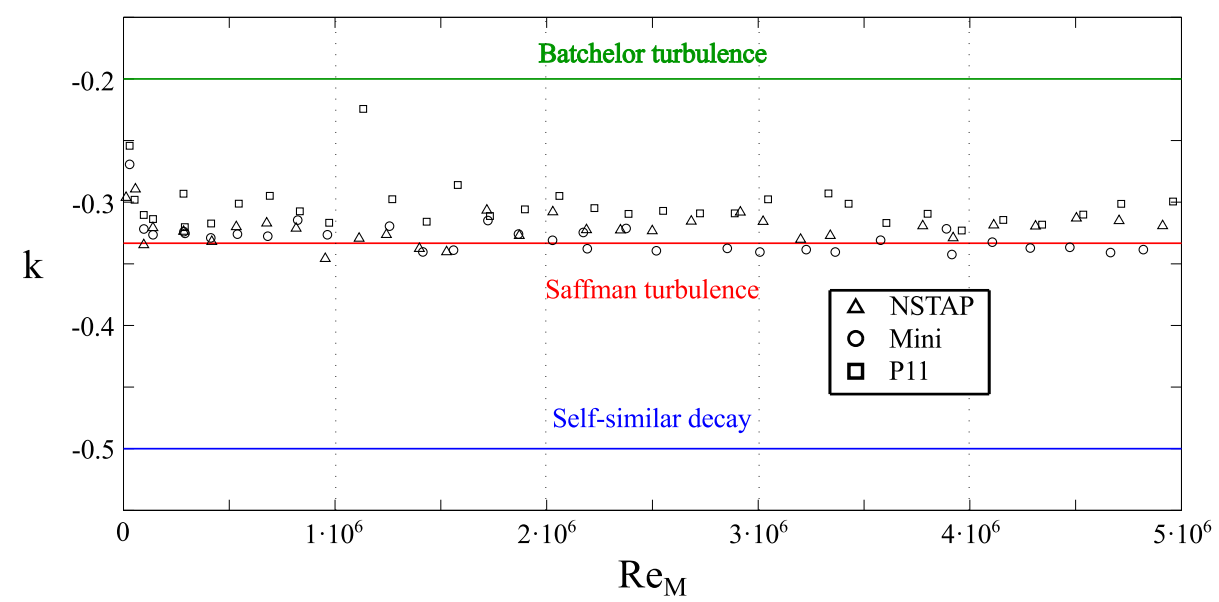

Figure 3.12: Virtual origin independent decay exponents $k$ from the turbulentintrinsic description $L \propto\left(u^{2}\right)^{k}$. The colored lines represent the predictions of the classical decay models.

Even though the decay process is largely dominated by the structure of the large scales, the physics is closely related to the small-scale structure of the flow. This can 
easily be seen by going back one step to the classical equation (1.32). It relates the behavior of the large scale $L$ to the energy dissipation rate $\varepsilon$, which is by definition a quantity of the small scales,

$$
\varepsilon=\frac{v}{2} \sum_{i, j}\left(\frac{\partial u_{i}}{\partial x_{j}}+\frac{\partial u_{j}}{\partial x_{i}}\right)^{2}
$$

Figure 3.13 shows the constant $C_{\varepsilon}=\varepsilon L / u^{3}$ for all experiments in the dataset Decay. To calculate the energy dissipation rate, the left-hand side of equation (1.32), $\frac{3}{2} \frac{\mathrm{d} u^{2}}{\mathrm{~d} t}=-\varepsilon$, was used, the derivative was estimated by means of the aforementioned finite difference formula for unequal sub-intervals, as applied to the individual fitted power-laws of the decay curves. This circumvents the need to compute velocity derivatives directly, which is not feasible for significant parts of the taken measurements, where the probe size is larger than the Kolmogorov length scale (see table 2.3).

In figure 3.13 , one can see that $C_{\varepsilon}$ is a constant of order one, varying only slowly with the distance from the grid. The individual curves scatter around the median curve, plotted as a bold black line, without any apparent trend in Reynolds number. The scatter arises from the difficulty of obtaining the energy dissipation rate and integral length scale for the short datasets having relatively limited statistics. The classical predictions of Kolmogorov (1941c) hold reasonably well, justifying the analysis in figure 3.11 and thus the Reynolds number independence of the decay of turbulence. 


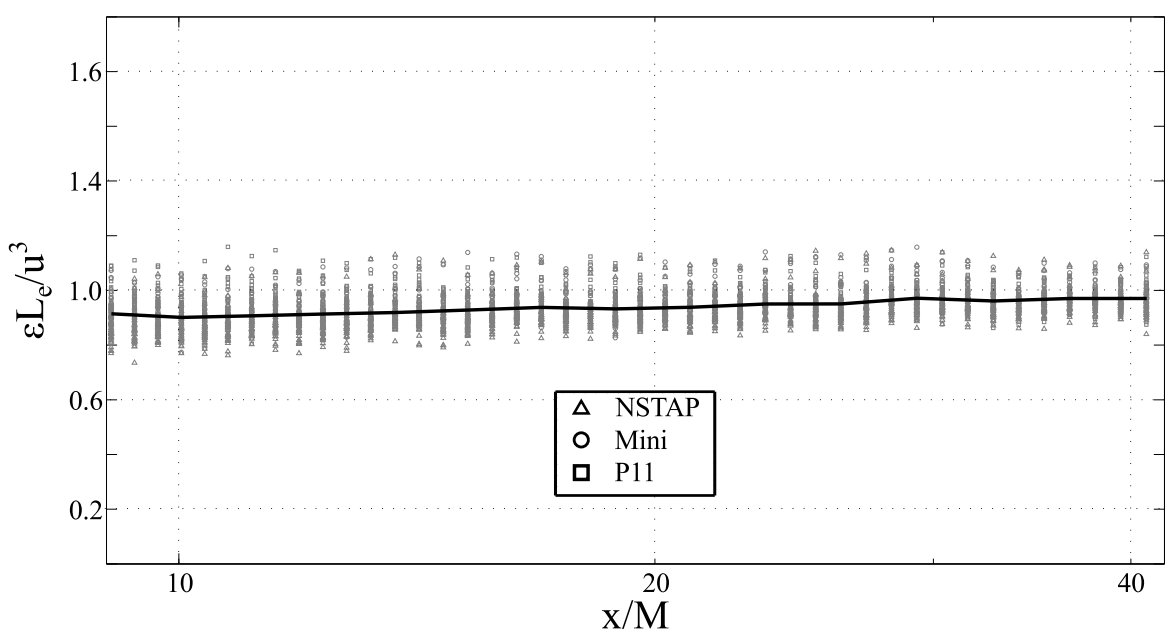

Figure 3.13: Semilogarithmic plot of the constant $C_{\varepsilon}$ versus the distance from the grid $x / M$. The light gray points represent experimental data from the dataset Decay, the bold black line the non-discriminated median over logarithmically spaced bins. $C_{\varepsilon}$ is only a slowly varying variable with time and is of order one. The lowest Reynolds-number experiments give substantially different values for $C_{\varepsilon}$, originating in the difficulty to determine the precise values of $L_{e}$ and $\varepsilon$ especially with $\mathrm{P} 11$ probes in experimental conditions at very low Reynolds numbers. These experiments are thus excluded from this figure.

\subsubsection{The Energy Spectrum}

In the previous section, it was shown that there is a close connection between the decay, dominated by the large scales, and the energy dissipation, a small-scale quantity. A more detailed investigation of this connection can be done using the time progression of the energy spectrum $E(k, t)$, covering all scales of motion. Singlewire hot-wires are not able to measure more than one velocity component, in these experiments the longitudinal velocity component, so that the three-dimensional energy spectrum is not directly measurable. However, using Taylor's hypothesis, 
is it at least possible to compute the longitudinal velocity correlation function $C(r)=\langle u(x+r) u(x)\rangle$, from which the one-dimensional energy spectrum

$$
E_{11}\left(k_{1}\right)=\frac{2}{\pi}\left\langle u^{2}\right\rangle \int_{0}^{\infty} C(r) \cos \left(k_{1} r\right) \mathrm{d} r
$$

can be computed (Taylor 1938, Pope 2000). In the inertial range, the onedimensional energy spectrum scales identically as the three-dimensional spectrum, with $E_{11}\left(k_{1}\right) \propto k^{-5 / 3}$. For large scales, i.e. $k \rightarrow 0$, the one-dimensional longitudinal energy spectrum becomes a constant of $k, E_{11}\left(k_{1}\right) \propto k^{0}$. Figure 3.14 shows the compensated longitudinal energy spectra at different distances from the grid for an intermediate-Reynolds-number experiment measured with an NSTAP.

In the inertial range, all spectra indeed approach the expected scaling of $E_{11}\left(k_{1}\right) \propto$ $k^{-5 / 3}$, corresponding to a horizontal line in the compensated representation. Being independent on the distance from the grid, this behavior is universal during the decay process. Two distinct features can be observed on the far ends of the spectrum, both of them highlighted in figure 3.15 .

The general shape of the spectrum at the large scales is preserved during the decay, agreeing with the common theories. The time-dependent growing local maximum in the left part of figure 3.15 might be related to boundary effects starting to affect the flow at very large scales at the end of the wind tunnel. As visible in the right part of figure 3.15, the drop-off of the energy spectrum moves to larger length scales with time, indicating that the small scales react first during the decay process. 


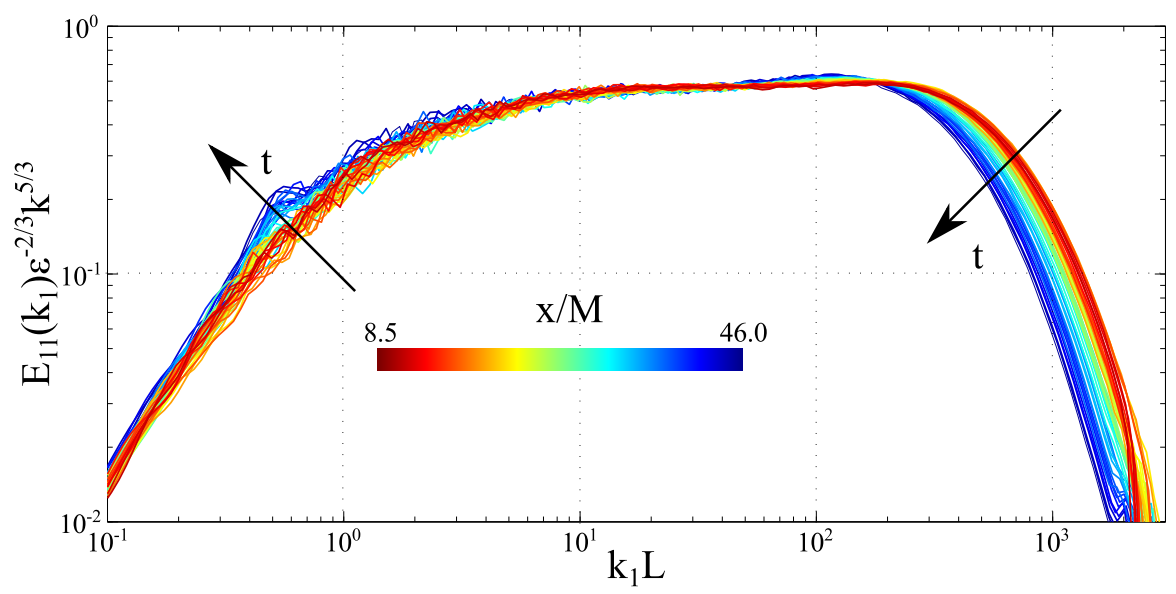

Figure 3.14: The compensated one-dimensional longitudinal energy spectra on a $\log -\log$ plot for one decay measurement at 5 bar $\mathrm{SF}_{6}$. The data was acquired with an NSTAP. The figure shows the energy spectra for all 50 logarithmically spaced distances from the grid, colored from blue (furthest downstream position, large times) to red (closest position to the grid, short times). Note that $L$ is a quantity of time, as it grows during the decay. The abscissa is normalized by the time dependent integral length scale based upon equation (1.15).

This has to be understood in a statistical manner. Close to the grid, there is a certain distribution of scales in the turbulent flow. While being swept downstream of the tunnel and decaying, the distribution of scales changes such that the smallest scales are removed from the distribution by dissipation. This leads to a shrinking inertial range.

This effect can directly be seen in the development of the scales of turbulent during the decay. Figure 3.16 shows the normalized evolution of the integral length scale, $L_{e}$, and the Kolmogorov length scale, $\eta$, as a function of the distance from the grid. The Kolmogorov length scale grows significantly faster than the integral length scale, consistent with the description above. 

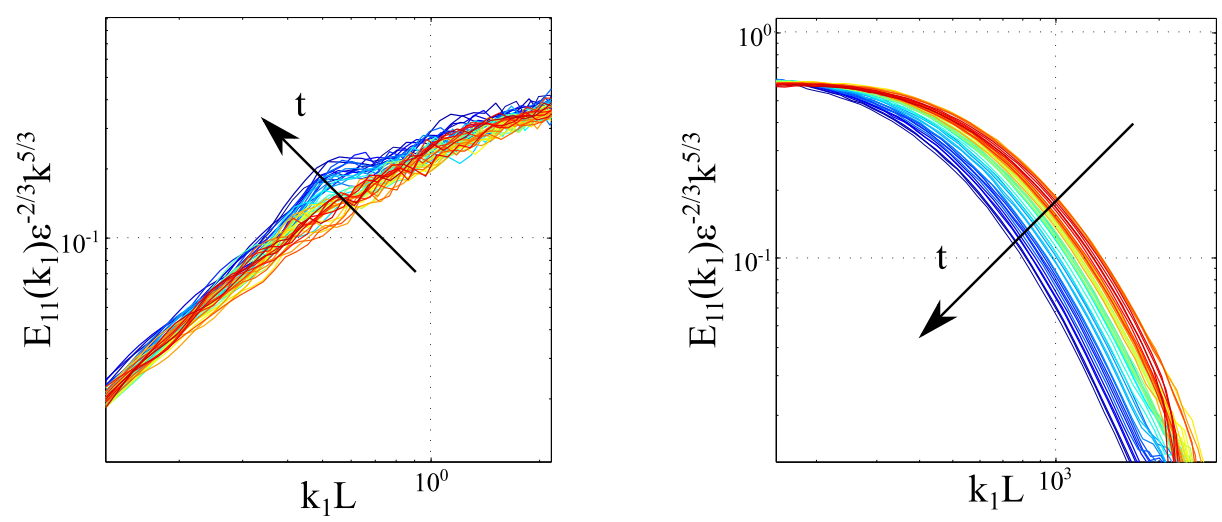

Figure 3.15: The energy spectra from figure 3.14 are shown zoomed into the large scale (left) and small scale (right) time dependent features of the curves. Color coding is analogous to as in figure 3.14.

This has been independently measured by, e.g., di Lorenzo (2014), who measured the real-time evolution of decaying turbulence in the so-called Lagrangian Exploration Module (Zimmermann et al. 2010). The turbulent flow in this icosahedronshaped water container is driven by twelve individually controllable propellers. By measuring the trajectories of Lagrangian particles after turning off the turbulenceproducing propellers, di Lorenzo was able to explicitly show that in a freely decaying turbulent flow, the small scales dissipate energy faster than the rate at which energy is injected into them from the larger scales. While the large scales determine how fast the energy decays, the conservation of large-scale physical quantities like, e.g., the linear or angular momentum results in a decay that reacts at the small scales first.

The major theories on decaying turbulence utilize conserved quantities of motion in order to relate the decay exponent to the large-scale part of the three-dimensional 


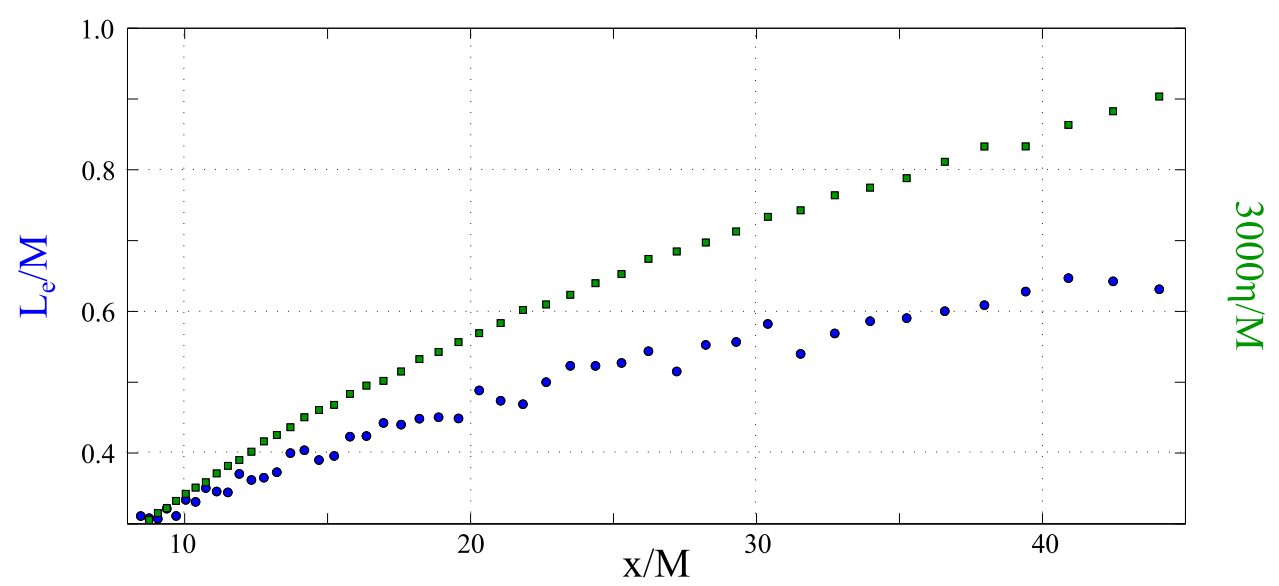

Figure 3.16: The normalized integral length scale (blue circles) and the Kolmogorov length scale (green squares) as a function of the distance from the grid. The Kolmogorov length scale grows significantly faster than the integral length scale.

energy spectrum. Analyzing instead the large-scale part the one-dimensional longitudinal energy spectrum is not useful, as it follows the trivial large-scale scaling of $E_{11}\left(k_{1}\right) \propto k^{0}$. Furthermore, even obtaining an accurate estimation of the large-scale part of the spectrum $E_{11}\left(k_{1}\right)$ is highly non-trivial, as this quantity is calculated as an integral over the velocity autocorrelation function, which is subject to increasing statistical noise at large separations. Disregarding problematic noise, it is in principle possible to calculate the full three-dimensional energy spectrum from knowledge of the one-dimensional longitudinal energy spectrum alone under the assumptions of isotropy. The three-dimensional spectrum is found to be related to the one-dimensional spectrum via a modified second derivative (Pope 2000, p. 227): 


$$
E(k)=\frac{1}{2} k^{3} \frac{\mathrm{d}}{\mathrm{d} k}\left(\frac{1}{k} \frac{\mathrm{d} E_{11}(k)}{\mathrm{d} k}\right)
$$

As mentioned above, the statistical noise in the large-scale part of the onedimensional longitudinal energy spectrum is not negligible, so calculating the three-dimensional energy spectrum directly from equation (3.13) produces a highly noise-dominated curve without meaningful information at the large scales. With the data from the VDTT it is however possible to extract this information. The individual computations of the energy spectrum from the decay measurements, which are each 5 minutes long and thus contain $1.8 \cdot 10^{7}$ individual samples of the velocity, are not long enough to get reliable large-scale information. But, as the quantity of interest is not the whole three-dimensional energy spectrum but rather only its large-scale behavior, is a possibility to get an adequate estimation of its scaling properties. Along the lines of the results from figures 3.7, 3.11, 3.13 and 3.14, the large-scale part of the energy spectrum can be assumed not to change significantly with either the Reynolds number or the distance from the grid. It is therefore possible to combine the whole time series of the dataset Decay into one three-dimensional large-scale energy spectrum. Assuming that only statistical noise causes problems in calculating equation (3.13), following the representation in figure 3.11 , by calculating the three-dimensional spectra from the individual one-dimensional spectra, one produces an ensemble of noise-dominated threedimensional spectra. The median of the noise-dominated three-dimensional energy 
spectra then gives an estimation of the physics of the large-scale part of the flow. By doing this, the amount of available data can be increased by almost four orders of magnitude from $1.8 \cdot 10^{7}$ samples of the velocity to $6.5 \cdot 10^{11}$ samples by using the data from all pressures, probes ${ }^{1}$ and distances. This amount of data exceeds any comparable grid turbulence experiment by at least one order of magnitude. For example, the experiments from Krogstad \& Davidson (2010) only consisted of a total of approximately $10^{7}$ samples of the velocity. Figure 3.17 shows the results from computing (3.13) for the available P11 and NSTAP data.

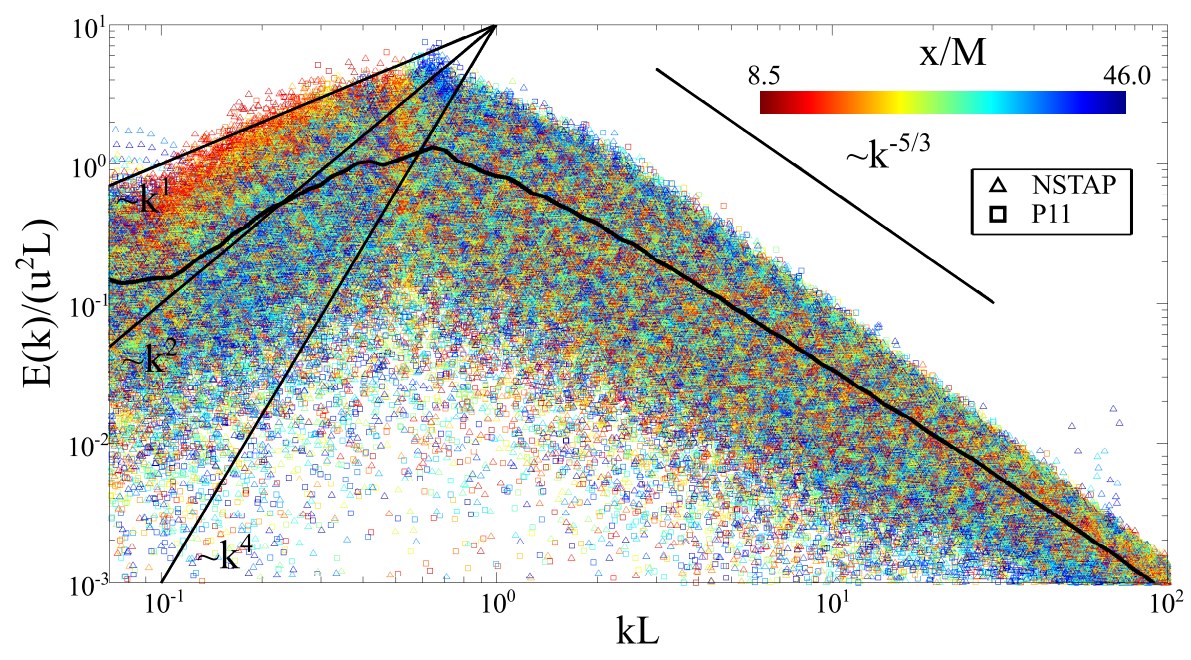

Figure 3.17: Three-dimensional energy spectra computed from the measured onedimensional longitudinal energy spectra in a logarithmic plot. The abscissa is normalized by the integral length scale of the individual measurements. The color code indicates the distance from the grid. Kolmogorov's prediction of $E(k) \propto k^{-5 / 3}$ in the inertial range is give as well as the predicted large-scale behavior of $E(k) \propto k^{1}$ (self-similar decay), $E(k) \propto k^{2}$ (Saffman) and $E(k) \propto k^{4}$ (Batchelor). The solid black line is the median over all individual curves.

\footnotetext{
${ }^{1}$ The data from the Mini probe is excluded from the plot as it shows wall effects at scales larger
} than the integral length scale. The probe is located closer to the tunnel floor than the other probes. 
In the inertial range, the averaged spectrum agrees with the classical prediction of Kolmogorov's theory. At a scale of approximately $2 L$, a transition towards a positive scaling exponent can be seen. From classical theory, given a decay exponent of $n=1.18$, the energy spectrum should scale with $m=(2 n) /(2-n)-1=1.88$, which, in contrast to the theoretical predictions, is not visible in figure 3.17. The change in scaling at scales starting at $10 \mathrm{~L}$ towards a shallower spectrum must be attributed to side wall effects. Structures of the size of $10 L$ at the end of the test section are of the length scale of the tunnel diameter itself, so the assumptions of homogeneity and isotropy break down for these sizes. As the conditions are needed to derive equation (3.13), the computed three-dimensional spectrum does not reflect physical reality. Within the limited accuracy, the degree of isotropy and the amount of data, the classically predicted connection between the large-scale part of the spectrum and the decay exponent seems to be not very well fulfilled. This is regardless of the Reynolds number or the distance from the grid, thereby possibly putting in question the picture of conserved large-scale conservation quantities determining the energy decay rate.

\subsubsection{Inertial-Range Effects}

The previous findings support the picture that large-scale structures govern the energy decay rate. Energy is removed from the system at the small scales where dissipation happens, while keeping the large-scale energy spectrum intact during the decay process. The intermediate inertial-range scales in this picture should not be affected by the decay of turbulence as long as the Reynolds number does not 
become too small. This can be confirmed using Kolmogorov's four-fifths law for the third-order structure function (see equation 1.25). The normalized third-order structure functions for a decay measurement in $\mathrm{SF}_{6}$ at 7 bar is shown in figure 3.18.

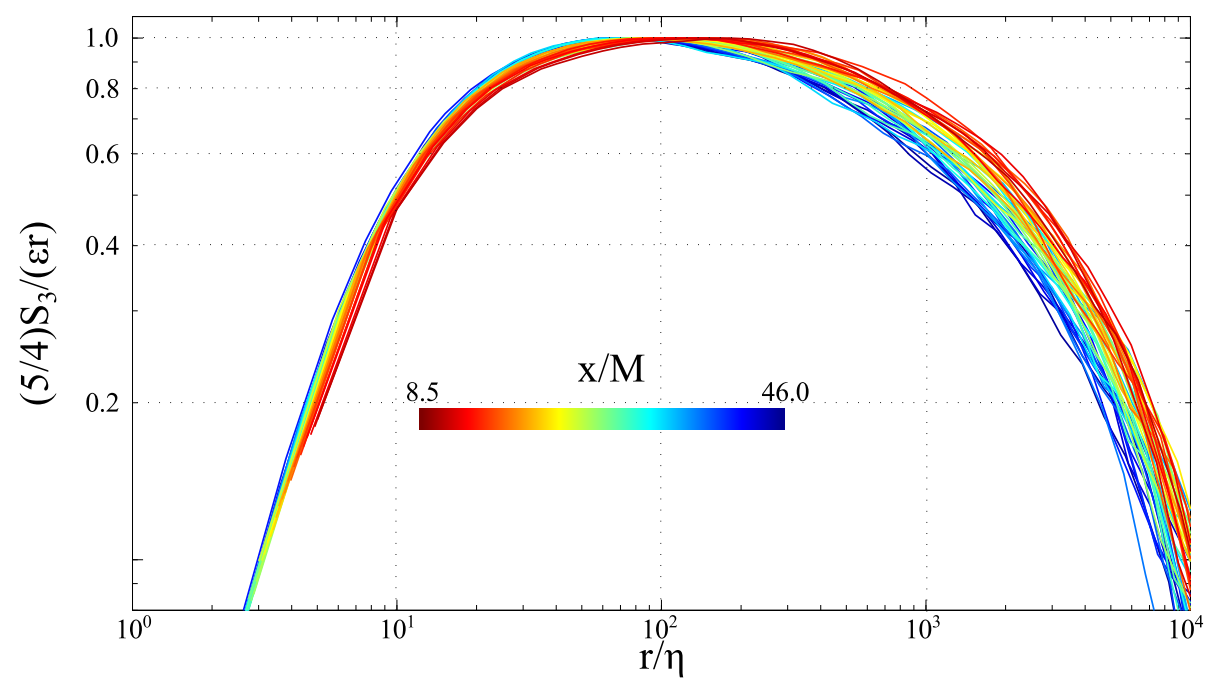

Figure 3.18: Normalized third-order structure functions measured in $\mathrm{SF}_{6}$ at 7 bar with an NSTAP. The abscissa is normalized by the Kolmogorov length scale $\eta$ at the respective distance from the grid. The color code contains information about the distance of the measurement to the grid. The extending inertial range stems from the decrease of Reynolds number with increasing distance from the grid.

From figure 3.18 there is an apparent widening of the inertial range with decreasing distance to the grid. This feature must be attributed to the change of Reynolds number during the decay alone. As the decay is not self-similar, the Reynolds number does decrease during the decay process, in this case from $R_{\lambda}=1200$ close to the grid down to $R_{\lambda}=930$ at the end of the measurement section. Figure 3.19 shows the decay of Reynolds number as a function of distance from the grid for the 
same measurement in $\mathrm{SF}_{6}$ at 7 bar as above. This significant change in Reynolds number causes the broadening of the structure functions. Apart from this, there is no apparent feature emerging during the decay. Thus, the picture that only the large scales determine the conditions of the decay is supported. Details as to how well Kolmogorov's four-fifths law is fulfilled in general will be discussed in chapter 4 .

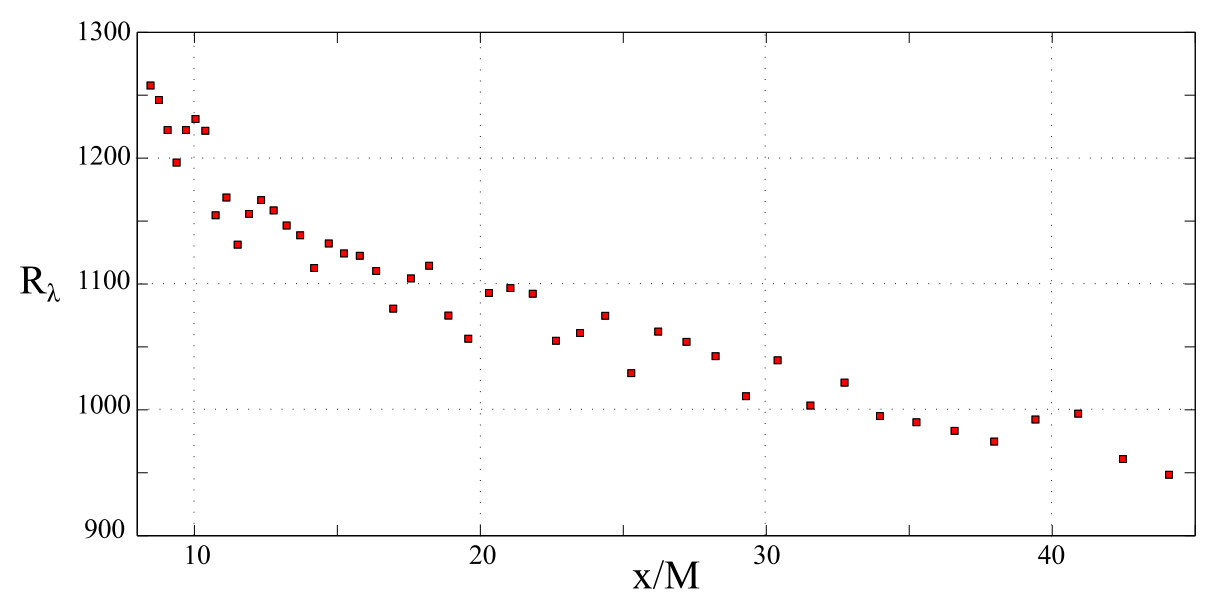

Figure 3.19: Decay of the Taylor scale Reynolds number as a function of distance from the grid for an NSTAP measurement in $\mathrm{SF}_{6}$ at 7 bar.

\subsection{Effect of Grid Modifications}

The Navier-Stokes equations are a set of nonlinear differential equations, describing the time evolution of a turbulent velocity field $\mathbf{u}(\mathbf{x}, t)$ in a domain $\Omega$. Given initial conditions $\mathbf{u}(\mathbf{x}, 0)=\mathbf{u}_{0}$ for $\mathbf{x} \in \Omega$, boundary conditions on the surface of $\Omega$, $\mathbf{u}\left(\left.\mathbf{x}\right|_{\delta \Omega}, t\right)=\mathbf{u}_{\delta}(t)$, and the Reynolds number Re, the equations predict the behavior of the velocity field for all times. In real flows, confined by solid walls, the boundary 
conditions are of Dirichlet type, so that $\mathbf{u}\left(\left.\mathbf{x}\right|_{\delta \Omega}, t\right)=0$ for all times. As the details of the initial condition $\mathbf{u}_{0}$ are not controllable in experiments, the deterministically chaotic nature of turbulence will produce inherently different time evolutions of the flow for even the slightest variations of $\mathbf{u}_{0}$. Initial conditions are only able to be reproduced in a statistically averaged sense, which is done in the VDTT based upon the mean speed of the flow. The analysis of the decay process, so far, has focused on a disentanglement of the Reynolds number and boundary conditions by only changing the viscosity between experiments and keeping the averaged initial condition constant to the best possible extent. This approach resulted in a description of decaying turbulence, focusing purely on the Reynolds-number dependence and finding that this parameter does not influence the rate at which energy decays. As there is a tight connection between the large-scale structure of the flow and the rate of decay, a change in the production of turbulence might lead to a different type of decay.
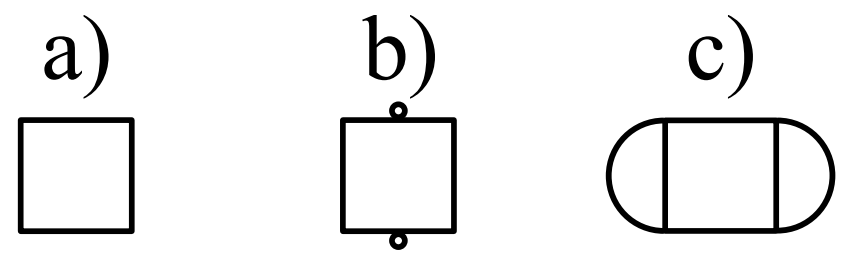

Figure 3.20: Schematic of the grid modifications. a) Standard grid bars. b) Wirewrapped grid bars. c) Rounded grid bars.

This change of production is realized by a change of grid geometry, and thus, in a mathematical description, by a change of $\delta \Omega$ of the fluid domain. In contrast to the one-dimensional, Reynolds number, the surface of the fluid domain is a quantity with infinite degrees of freedom, obviously not accessible as a fully controllable 
experimental parameter. It is, however, possible to test the results on the Reynoldsnumber dependence of decaying turbulence regarding their robustness to changes in $\delta \Omega$. Altering the shape of the grid bars, for example, changes the way turbulent wakes detach from them and can significantly alter the flow. A schematic of the modifications of the grid bars is shown in figure 3.20. The classical grid in dataset Decay used rectangular grid bars. The grids of the dataset Decay_Modified used partially wire-wrapped grid bars and rounded grid bars (see section 2.4.3).

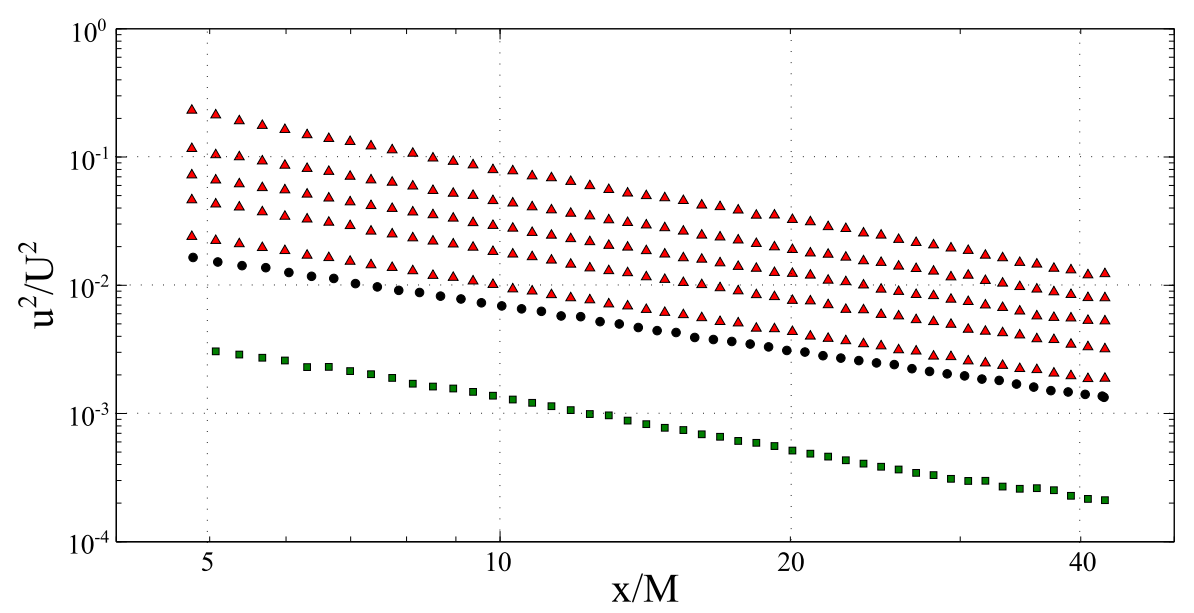

Figure 3.21: The decay of turbulent kinetic energy for different grid geometries at various Reynolds numbers. The data was obtained using a Mini probe, black circles correspond to the classical grid, red triangles to wire-wrapped grid bars, green squares to rounded grid bars. The top four curves are shifted vertically for better visibility. The Reynolds numbers are (from bottom to top) are $\operatorname{Re}_{M}=$ $6.0 \cdot 10^{4}, 2.9 \cdot 10^{4}, 3.1 \cdot 10^{5}, 5.8 \cdot 10^{5}, 1.2 \cdot 10^{6}, 2.4 \cdot 10^{6}$ and $\operatorname{Re}_{M}=4.8 \cdot 10^{6}$.

For the wire-wrapped grid, decay measurements were conducted between 1 bar of air and 15 bar of $\mathrm{SF}_{6}$ to investigate a possible change in Reynolds number behavior. With the rounded grid bars, the decay rate of air turbulence at atmospheric pressure was measured to test the robustness of the intrinsic structure of the turbulent flow 
with respect to the production process. Figure 3.21 shows selected decay curves obtained with a Mini probe. Like with the original grid, the decay of energy seems to follow a power-law with no apparent Reynolds-number dependence. Note that the turbulence intensity of the grid with rounded bars is significantly lower than that of the wire-wrapped grid. A change in the way the wakes detach from the grid bar might, in principle, influence the time needed to form a homogeneous, isotropic flow. This could, then, result in a changed virtual origin with a possible Reynolds-number dependence due to effects equivalent to a drag crisis.

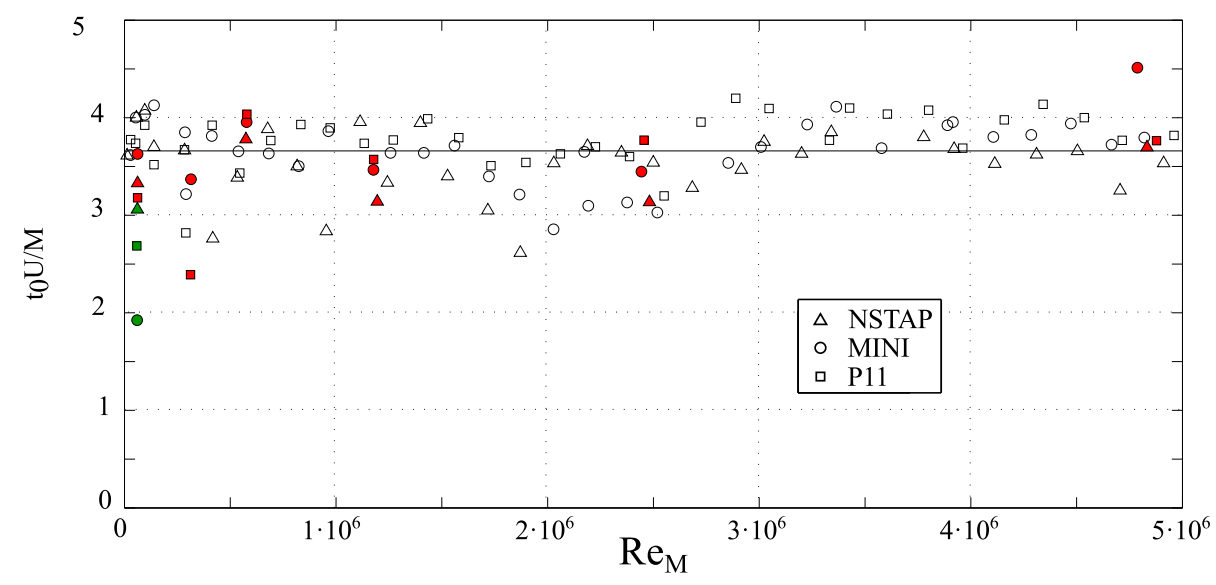

Figure 3.22: Virtual origin from a three-parameter fit to the data. The black symbols correspond to the original data from figure 3.2, red symbols to data from the wire-wrapped grid and green symbols to the rounded grid bars.

Figure 3.22 shows the results from three-parameter power-law fits to the decay curves, analogous to the procedure in section 3.1.2. The black symbols correspond to the results from the classical grid and the colored symbols to the modified grid geometries. The virtual origin obtained for the flow behind the wire-wrapped grid are of the same order as the ones of the original grid, with no apparent Reynolds- 
number dependence. The virtual origins for the measurements behind the grid with rounded grid bars are slightly smaller than the global mean of $x_{0}=3.66$, hinting towards a change of turbulence production. This is in agreement with the significant drop in turbulence intensity shown in figure 3.21. Applying the same analysis as in section 3.1.2, a two-parameter fit to the decay curves using the globally averaged virtual origin results in the decay exponents shown in figure 3.23.

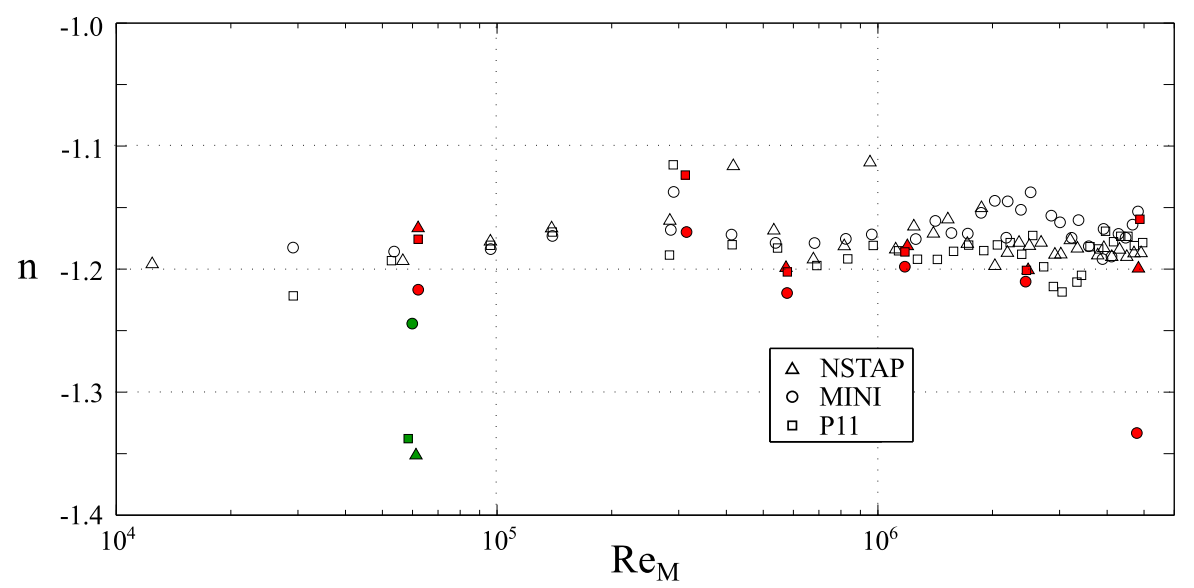

Figure 3.23: The decay exponents from a two-parameter fit to the data with fixed virtual origin. The results from the wire-wrapped grid are represented by red symbols, from the rounded grid with green symbols, black symbols correspond to the data in figure 3.7 from the classical grid.

The modification towards a wire-wrapped grid has little influence on the rate of decay, which is still independent of Reynolds number. Within the scatter of the data, the results of both the classical and the wire-wrapped grid, give agreeing results of a decay exponent in the vicinity of Saffman's prediction. The exponents of the decay measurement behind the rounded grid bars are notably lower, lying between Saffman's prediction of $n=-1.2$ and Kolmogorov's prediction of $n=-10 / 7$. 
However, the large scatter between the three hot-wire probes, stemming from the low turbulence intensity produced by this modified grid, make a definite statement about the type of decay for the rounded grid bars difficult from this analysis alone. Along the lines of the arguments in section 3.1.2, the large-scale structure of the flow will determine the rate of decay. Figure 3.24 shows the normalized integral length scale versus the normalized turbulent kinetic energy in a logarithmic plot analogous to figure 3.11. Black symbols correspond to the data from the original classical grid in figure 3.11, red symbols to the wire-wrapped grid and green symbols to the rounded grid. This figure shows that the large-scale structure of the flow and its Reynolds-number independence is robust towards changes of the boundary $\delta \Omega$ in the sense that non-negligible changes of the grid geometry do not result in drastically different large-scale behavior. The flow behind the partially wire-wrapped grid behaves identically to the classical grid flow with respect to the Reynolds number. 


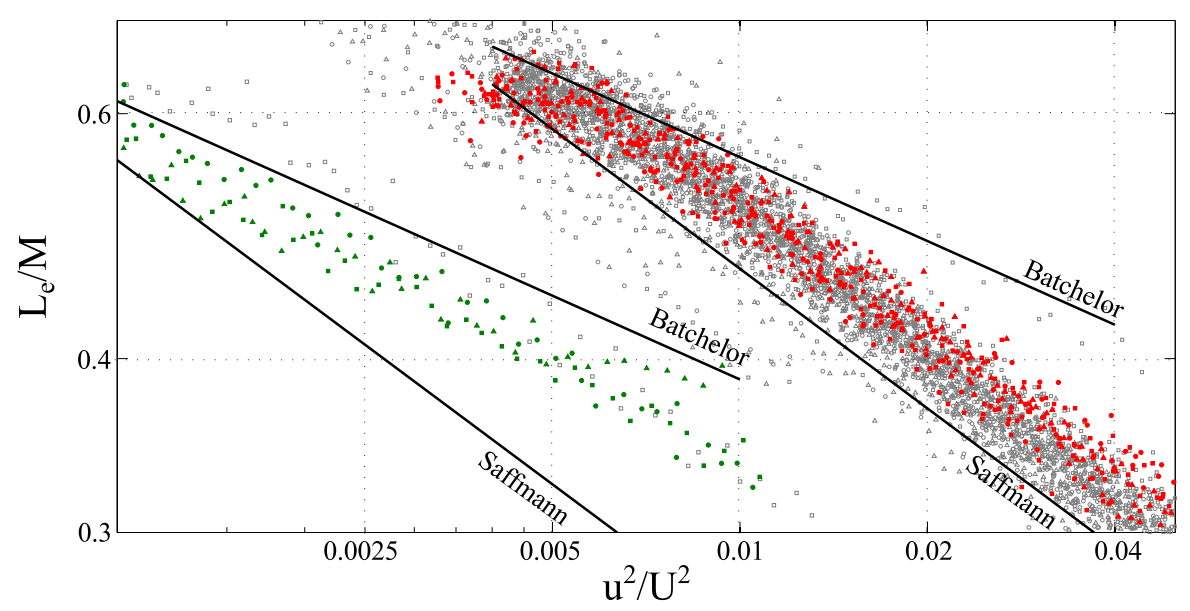

Figure 3.24: Logarithmic plot of the normalized integral length scale versus the normalized turbulent kinetic energy for all three grid variations. The data from the wire-wrapped grid (red) and the classical grid (black) show identical intrinsic large-scale behavior. The rounded grid (green) produces a significantly lower turbulence intensity along with a slightly shallower power-law dependence of $L$ with regards to $u^{2}$ due to the low Reynolds number.

The low turbulence intensity and the low Taylor-scale Reynolds number of the flow behind rounded grid bars shifts the green data in the $L$ vs $u^{2}$ curves to the left and leads to a slightly shallower relation between $u^{2}$ and $L$. This can be understood in the expected low-Reynolds number approach towards more negative decay exponents (see figure 3.10). As mentioned earlier, the dependence of the decay exponent on the boundary conditions, i.e., the geometry of the grid, is by definition an infinite-dimensional problem which cannot be solved in full detail by either experiment or numerical simulation. The results from the VDDT decay experiments, however, strongly support Saffman-type turbulence as the generic, high-Reynolds-number type of turbulence for the flow behind passive grids along with absence of an approach towards a self-similar decay. 


\subsection{Measurement Uncertainties}

To conclude this chapter, some remarks on the uncertainties in determining the decay exponent are necessary. As seen, e.g., in figure 3.7, the obtained decay exponents scatter moderately around their mean value of $n=1.18 \pm 0.02$. The corresponding prefactors in figure 3.9 show significantly larger scatter. To explain this difference, one has to separate the effects of random errors and systematic errors. Random errors can be calculated directly from the fitted model parameters themselves to give an impression about the statistical noise in the data. Figure 3.25 gives the standard errors and the $95 \%$ confidence intervals of the fitted exponents of the dataset Decay, assuming normal distributed errors. The standard errors are defined as the square root of the trace of the variance-covariance matrix of the fitted parameters. The scatter of the data around its mean value is of the order of the errors of the individual exponents for the bulk of the data, with several exceptions deviating significantly further from the mean than their error bars.

An independent method of estimating the quality of the fitted parameters is to use a method similar to bootstrapping (Efron 1979). This can be done by randomly removing individual points in the energy versus distance curve and refitting the sparser data using the same routine as with the full dataset. If iterated for multiple different random removals and number of randomly removed points, it is possible to extract information about the significance of the amount of available data. This is applied in figure 3.26. For an NSTAP dataset at $\operatorname{Re}_{M}=2 \cdot 10^{6}$, between 1 and 45 of the 50 measurement points where randomly removed from the dataset and the 

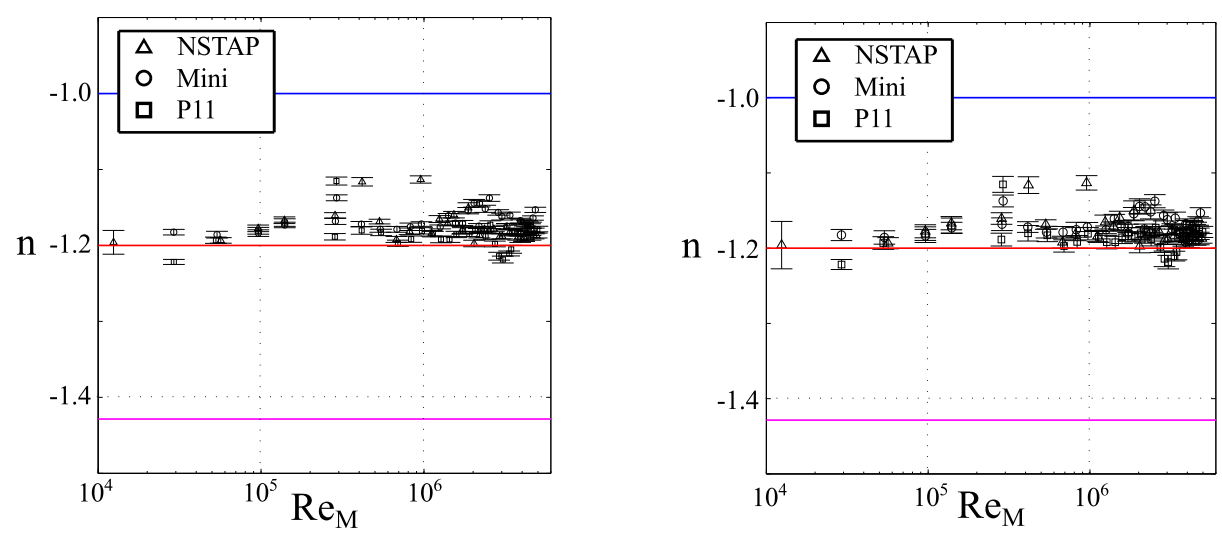

Figure 3.25: Direct computation of uncertainties from the fitting algorithm in semilogarithmic plots. The left figure shows the standard errors (see section 4.5) of the individually fitted exponents for all decay experiments in the dataset Decay. The right figure gives the corresponding 95\% confidence intervals. The scatter of the data is slightly larger than the confidence intervals. The colored horizontal lines represent the predictions of self-similar, Saffman and Batchelor turbulence as in figure 3.7.

nonlinear least squares algorithm was used to extract the decay exponents of the shortened datasets. This was repeated 20 times for each number of removed points. The resulting decay exponent is given in figure 3.26.

It can be observed first of all that the scatter in the resulting decay exponents grows with the number of removed points, as it has to be expected. Furthermore, for a small number of removed points, the scatter in the resulting exponents is significantly smaller than the computed standard errors in figure 3.25. Additionally, there is no trend in the mean resulting exponent with respect to the number of removed points and there is thus no spurious effect on the exponents due to a possibly insufficient number of data points. It has to be noted, however, that the scatter in the exponent is non-zero and thus non-negligible even for a small number 


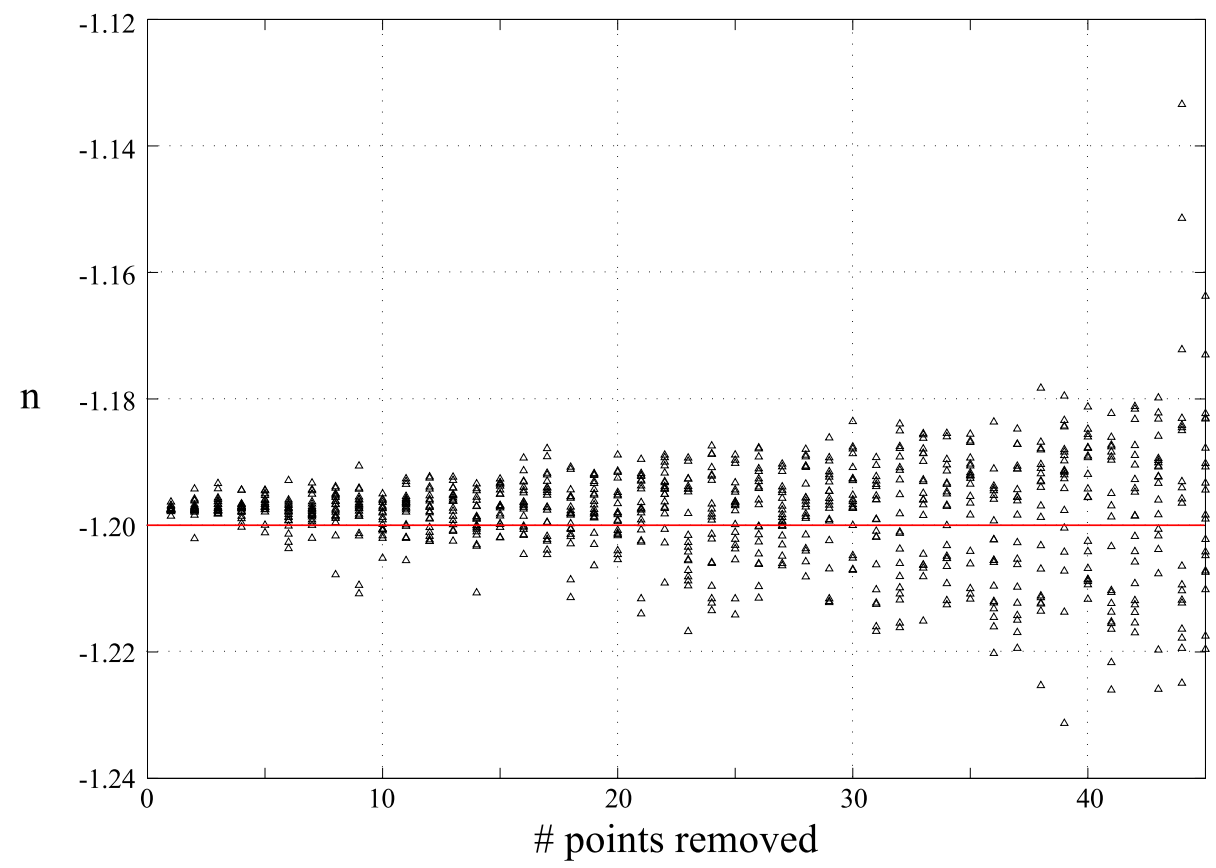

Figure 3.26: Scatter by random removal of measured points in the decay curves for a NSTAP measurement at $\operatorname{Re}_{M}=2 \cdot 10^{6}$. Shown is the result of the twoparameter fit for the decay exponent by randomly removing a set number between 1 and 45 of the 50 points. The procedure was iterated 20 times for each number of removed points to give a measure of the noise due to the limited amount of data.

of removed points. Even though the scatter is small, it contributes to the scatter in figure 3.7. This effect could be reduced by repeating the experiment at an even larger number of distances from the grid.

A priori, it is unclear whether systematic errors influence the measurement of the decay exponents. The most likely systematic error to influence the measurements of the decay rate is a faulty calibration. An incorrect calibration will lead to incorrect measurements of the magnitude of the velocity and thus velocity fluctuations and 


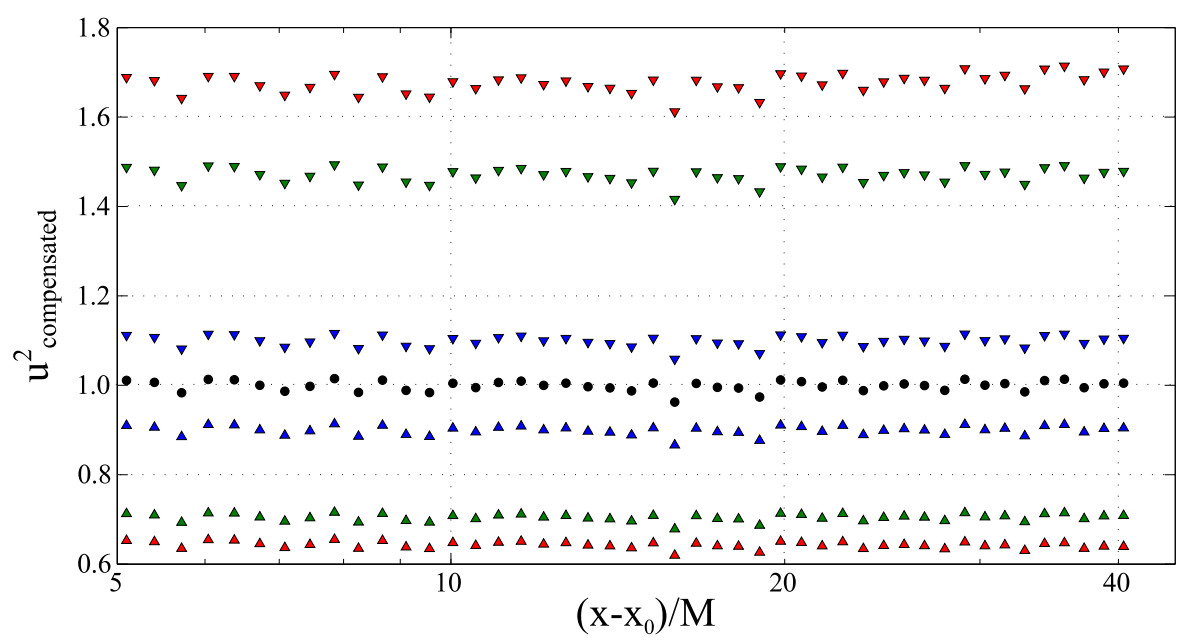

Figure 3.27: Effect of altered calibrations on the decay of non-normalized, compensated turbulent kinetic energy. Black circles correspond to the original calibration of a NSTAP measurement at $\operatorname{Re}_{M}=2 \cdot 10^{6}$. The colored triangles are results for the same measurement with artificially altered calibrations. Upwards triangles correspond to an increase of the corresponding parameter, downwards triangles to a decrease. Blue triangles correspond to calibrations in which the signal from the pressure transducer was changed by $10 \%$. For the green triangles, the prefactor $b$ in King's Law in equation (2.7) was changed by $10 \%$ after computing the coefficients. The red triangles correspond to a changes exponent in King's Law. The decay exponent is virtually invariant towards such changes in the calibration procedure.

the kinetic energy. Along this line of thought, mistakes in the calibration procedure should transfer to the measurement of the decay rate. To test the effects of the calibration on the computed fit parameters, several artificial modifications have been applied to an existing calibration at $\operatorname{Re}_{M}=2 \cdot 10^{6}$ to estimate the extent of the importance of the calibration for the fit parameters. Figure 3.27 shows the resulting compensated decay curves for specific alterations of the calibration. The curves are compensated by the results from the power-law fit to the original black 
curve. Blue triangles assume an incorrect measurement of the differential pressure by the pressure transducer. This directly corresponds to a change in the additive term in King's Law. The measured pressure difference of the pressure transducer was increased (upwards triangles) or decreased (downwards triangles) by $10 \%$ to simulate a significantly erroneous pressure transducer. The resulting curves are shifted due to a change of mean speed and thus changed magnitude of velocity fluctuations. This shift does, however, not come with a change in the slope. The resulting decay exponent is invariant towards this change in the pressure transducer signal. For the green triangles, the slope $b$ in the fitted King's Law in equation (2.7) was changed by $10 \%$ a posteriori. This still only results in a shift of the decay curve. As an estimate for the upper bound of uncertainty due to an erroneously estimated exponent in King's Law, the exponent was changed by $10 \%$ a posteriori as well for the red triangles. Like the other alterations, no change in the decay exponent can be observed. The decay exponent $n$ is, thus, a robust quantity with respect to the calibration. Systematic errors in the calibration procedure do not influence the results in figure 3.7 in a significant way. The calibration does, however, have a strong effect in terms of the prefactor in figure 3.9, which is sensitive to a shift in the measured decay curve. The amount of scatter in the determination of the prefactor can therefore be attributed partially to potential errors in the calibration. 


\section{Scaling in Turbulence}

In this chapter, I investigate the statistical properties of turbulence in the inertial range and discuss the absence of scaling in real flows. Section 4.1 gives an overview over the current state of theoretical modeling of inertial range statistics. In section 4.2, I explore the Reynolds number behavior of the inertial range statistics in terms of the structure functions. I apply the technique of Extended Self-Similarity in section 4.3 and discuss the limitations regarding well-defined scaling properties of turbulent flows in section 4.4. The measurement uncertainties are discussed in section 4.5.

\subsection{Scaling in Inertial-Range Statistics}

One of the key features of turbulent flows is the deviation of its statistics from Gaussian behavior. It is well known that turbulent flows produce extreme events significantly more often than expected from Gaussian statistics. Yet as of today, an exact prediction or description of these deviations is still missing. The occurrence of extreme events, such as wind gusts, is connected to the tails of the probability 
density function of the velocity increment that can be descried in terms of structure functions of increasing order. As discussed in section 1.3, the only exact result from the Navier-Stokes equations predicts the behavior of the third-order longitudinal structure function $S_{3}(r)=\left\langle(u(x)-u(x+r))^{3}\right\rangle$ (Kolmogorov 1941a). Assuming a self-similar internal structure of the flow, the scaling behavior of any arbitrary structure function with regard to the separation $r$ can be shown to behave as $S_{n}(r) \sim r^{n / 3}$. Even though turbulent flows are not self-similar but rather intermittent with strong deviations from Gaussianity, the existence of scaling properties of the structure functions with a scaling exponent $\zeta_{n}$ is commonly agreed on in virtually all existing theoretical models. The first refined scaling model, already presented in subsection 1.3.3, was Kolmogorov's K62 framework (Kolmogorov 1962). It allowed for intermittency by a log-normal distributed energy dissipation rate. The resulting scaling exponents in the $\mathrm{K} 62$ model, $\zeta_{n}=\frac{n}{3}-\frac{\mu}{18} n(n-3)$, are nonlinear functions in the order parameter $n$ and depend on the intermittency parameter $\mu$. Over the years, numerous additional models for the structure functions have been developed and shall be briefly recaptured here.

1. The $\beta$-model by Frisch et al. (1978) solely bases upon the inertial range statistics itself by applying concepts of fractal dimensions to turbulent flows. It does not take into account small-scale quantities, such as the distribution of energy dissipation rate. The line of thinking here is that there are active eddies during the decay that only occupy a fraction of the whole fluid space and passive eddies that do not contribute. The space-filling does depend on the size of the active eddies. With introduction of the 'self-similarity dimension' 
$D$ that measures the space-filling, the authors obtain a linear model for the scaling exponents:

$$
\zeta_{n}=\frac{1}{3}(3-D)(3-p)
$$

2. The linear $\beta$-model was refined by Benzi et al. (1984) into the random $\beta$ model. The space-filling factor, which is a global constant of the $\beta$-model, is replaced with a random variable at each cascade step. This gives a set of scaling exponent depending on the distribution of $\beta$ via

$$
\zeta_{n}=\frac{n}{3}-\log _{2}\left[\beta^{(1-n / 3)}\right]
$$

3. A more sophisticated model that is based on the assumption of fractality was developed by Meneveau \& Sreenivasan $(1987 a, b)$. The authors assume that during the cascade process an eddy of certain size breaks down into $2^{d}$ smaller eddies. In contrast to classical modeling, in the multifractal model the energy is not distributed equally between all of the smaller eddies. Instead a fraction $p_{1}$ of the energy is distributed equally between one half of the $2^{d}$ eddies and the remaining fraction $p_{2}=1-p_{1}$ equally among the other half of the eddies. The resulting scaling exponents can be calculated from the generalized dimension $D_{q}$ with 


$$
\begin{aligned}
\zeta_{n} & =\left(\frac{n}{3}-1\right) D_{n / 3}+1, \\
D_{q} & =\log _{2}\left(p_{1}^{q}+p_{2}^{q}\right)^{1 /(1-q)} .
\end{aligned}
$$

4. The $\gamma$-model by Andrews et al. (1989) is a refinement of the K62 model. The authors find that a log-normal distribution of the energy dissipation rate is not fully justified as it fails to agree with several experimental findings. In the construction of their model, they instead assume the energy dissipation rate to follow a $\gamma$-distribution. Defining $1+1 / c=(L / r)^{\mu}$, with the integral length scale $L$ and the intermittency parameter $\mu$, they obtain the scaling exponents

$$
\zeta_{n}=\frac{1}{3} n-\mu \frac{\log \left[\Gamma(c+n / 3) / c^{n / 3} \Gamma(c)\right]}{\log [1+1 / c]} .
$$

5. A similar approach was chosen by Kida (1991), who assumed a stable distribution for the energy dissipation rate instead of a log-normal or $\gamma$-distribution. The parameter $0<\alpha<2$ determines the shape of the probability density function with $\alpha=2$ corresponding to a normal distribution. The resulting scaling exponents of the log-stable model are given by

$$
\zeta_{n}=\frac{n}{3}-\mu \frac{(n / 3)^{\alpha}-n / 3}{2^{\alpha}-2}
$$


6. A parameter-free model based upon the small-scale fluctuations in turbulence was developed by She \& Lévêque (1994). The energy dissipation field is modeled by a hierarchy of dissipation moments down to the size of filaments in the fluid flow. Using dimensional arguments and anomalous scaling for the energy dissipation moments, the authors obtain the parameter-free scaling exponents of the She-Leveque model

$$
\zeta_{n}=\frac{n}{9}+2\left[1-\left(\frac{2}{3}\right)^{n / 3}\right]
$$

7. Dubrulle (1994) found that the distribution of energy in the She-Leveque model corresponds to a log-Poisson statistic of the local energy dissipation rate. In a generalization of the She-Leveque model, using the space filling factor of the random- $\beta$ model and a parameter $\Delta$, connected to the codimension of the dissipative structures, the scaling exponents can be calculated with regards to the third-order scaling exponent in the Dubrulle model as

$$
\frac{\zeta_{n}}{\zeta_{3}}=(1-\Delta) \frac{n}{3}+\frac{\Delta}{1-\beta}\left(1-\beta^{n / 3}\right)
$$

This wide variety in models for the statistics of turbulent flows in the inertial range reflects the lack of precise knowledge of the underlying physics. It is not clear, which of the presented models are an adequate description of the scaling behavior 
of structure functions. All of these models have in common that they predict scaling properties of the structure functions in the inertial range. However, the role of the key parameter in turbulence, the Reynolds number, is not directly included in any of the above models. This is in contradiction to several experiments that do find an explicit dependence of the scaling exponents on low Reynolds numbers (see Antonia et al. (2000)), making a careful study of the Reynolds-number effects obligatory.

\subsection{The Third-Order Structure Function}

As Kolmogorov's fourth-fifth law gives an exact result for the inertial range behavior of the third-order structure function in the limit of high Reynolds numbers, an investigation of the Reynolds number dependence of measured third-order structure functions can provide further insight. It seems to be a natural first step before considering the general scaling behavior of structure functions of arbitrary order. Figure 4.1 shows third-order structure functions from the dataset Statistic_Large obtained with NSTAPs between Reynolds numbers $\operatorname{Re}_{\lambda}=110$ and $\operatorname{Re}_{\lambda}=1450$.

The structure functions are normalized using the cube of the velocity fluctuations, $u^{\prime 3}$, and the integral length scale obtained from the velocity autocorrelation function, $L$, to collapse the curves at large separations. Qualitatively, a scaling according to Kolmogorov's four-fifth law can be observed to emerge in the inertial range with increasing Reynolds number. Once collapsed at the large scales, the inertial range extends to the even smaller scales with increasing Reynolds number, keeping the core shape of the curves preserved. A quantity closely related to the third- 


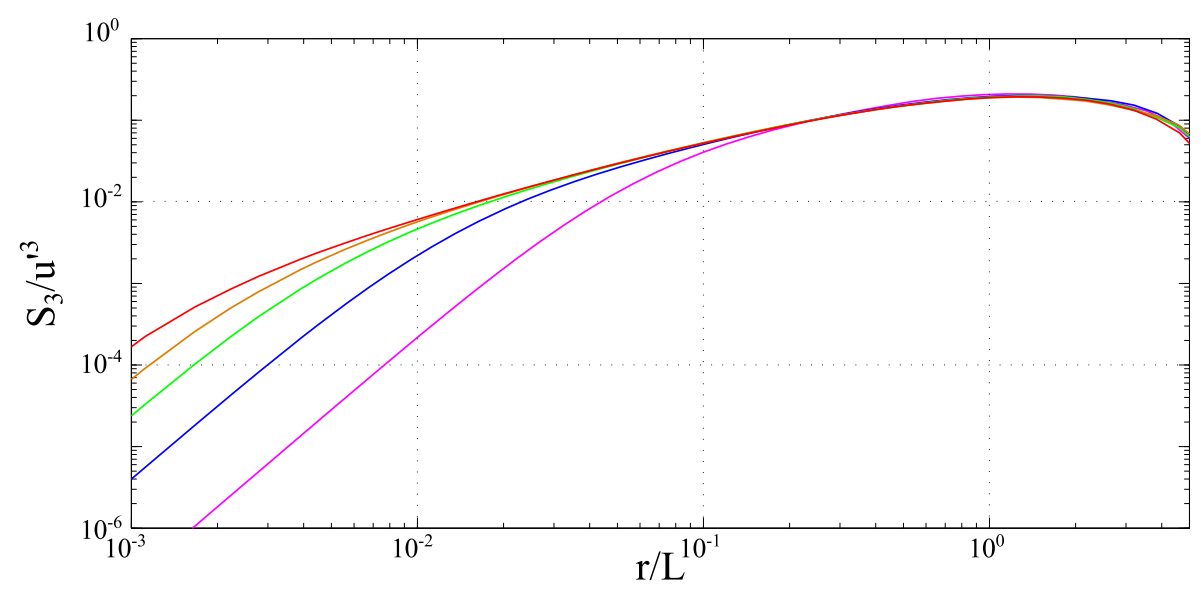

Figure 4.1: Normalized third-order structure functions versus the separation for the dataset Statistic_Large, measured with NSTAPs. By normalizing the abscissa by the integral length scales, the structure functions collapse at large scales. The curves stem from measurements at $R_{\lambda}=1450$ (red), $R_{\lambda}=920$ (orange), $R_{\lambda}=610$ (green), $R_{\lambda}=260$ (blue) and $R_{\lambda}=110$ (purple).

order structure function, the third-order structure function of the absolute value $S_{3 a}=\left\langle|\delta u|^{3}\right\rangle$, is shown in figure 4.2. The third-order structure function of the absolute value does not have a direct physical meaning but serves as a useful tool in section 4.3 and, thus, shall be introduced briefly here. $S_{3 a}$ preserves the emerging inertial-range scaling of the third-order structure functions with increasing Reynolds number, but also shows a converging, non-vanishing value at the large scales, due to its definition.

Typically, in order to better visualize the approach to the predicted inertial range scaling, the third-order structure function is plotted in a compensated way as $S_{3} / r$. This approach shall not be used here. While improving the estimate of whether scaling behavior emerges, such an approach can only be understood qualitatively 


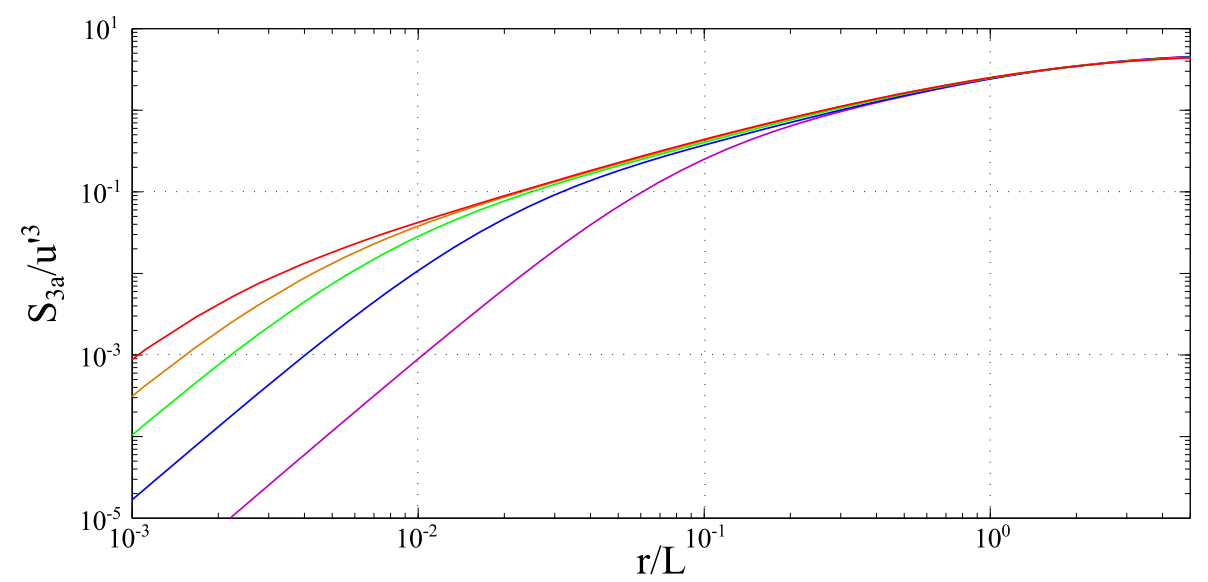

Figure 4.2: Normalized third-order structure functions of the absolute value versus the separation from the dataset Statistic_Large. The underlying data and color-coding is identical to figure 4.1.

and does not provide any quantitative insight. To extract quantitative information about the approach to scaling behavior in an inertial range, it is useful to introduce the logarithmic derivative of the third-order structure function. If the third-order structure function follows a power-law behavior, $S_{3}(r)=c \cdot r^{\zeta}$, then the logarithmic derivative returns the exponent of this power-law, $\left(\mathrm{d} \log S_{3}(r)\right) /(\mathrm{d} \log r)=\zeta$. In the special case of Kolmogorov's four-fifths law, $\zeta$ would be identically one. Figure 4.3 shows the logarithmic derivative of the third-order structure function at various Reynolds numbers from the datasets Statistic_Large and Statistic_Huge. The increased statistics form the longer datasets, in contrast to the dataset Statistic_Medium, is needed to collapse the large scales without noise contributions. The derivatives are computed with the same second-order finite differences algorithm as in chapter 3 (Singh \& Bhadauria 2009), so no a-priori smoothing oder modeling was applied. This prevents the occurrence of spurious information. 


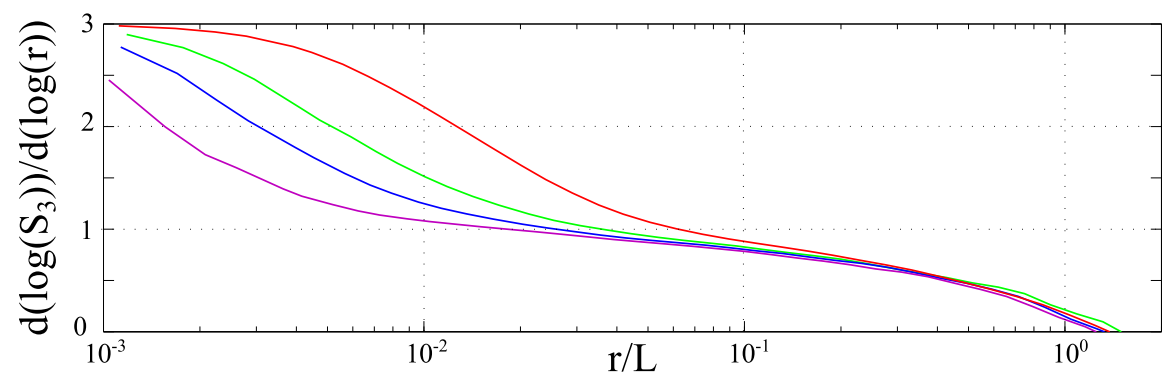

Figure 4.3: Logarithmic derivative of the third-order structure functions in a semi-logarithmic plot. The shown data are from measurements at $R_{\lambda}=300$ (red, Statistic_Huge), $R_{\lambda}=610$ (green, Statistic_Large), $R_{\lambda}=860$ (blue, Statistic_Large) and $R_{\lambda}=1320$ (purple, Statistic_Large) and measured with NSTAPs. The curves approach a dissipation-range scaling of 3, as expected. A plateau of value 1 in the inertial range would confirm K41 scaling, whereas a plateau of an arbitrary value would correspond to a power-law scaling with another exponent. Neither of these can be observed at any Reynolds number.

At the smallest scales, where the velocity field is smooth, the logarithmic derivatives approach the expected dissipation range scaling of $S_{3} \sim r^{3}$. At the large scales, regardless of Reynolds number, the curves collapse due to the normalization by the integral length scale $L$. At the intermediate scales, a power-law scaling of the third-order structure function would be represented by a plateau of the logarithmic derivatives. This feature is absent for even the highest Reynolds numbers. Instead, the logarithmic derivatives approach a curve with a systematic negative slope with increasing Reynolds number. As a consequence, there is no true power-law scaling in the third-order structure function even at the highest Reynolds numbers measured. And disregarding a possible transition towards different physics at even higher Reynolds numbers, an approach to such an power-law scaling seems improbable. The lack of a proper scaling behavior also implies that it is not possible to extract 
well-defined scaling exponents from the third-order structure function. This lack of scaling is not unique to the third-order structure function, but also carries on to other orders similarly. Figure 4.4 shows the logarithmic derivatives of the fourth-order structure function for the same datasets as in figure 4.3. In the K41 framework, self-similarity would demand a well-defined plateau at a value $4 / 3$, whereas the various models presented in section 4.1 would by confirmed by a plateau at their corresponding predicted value.

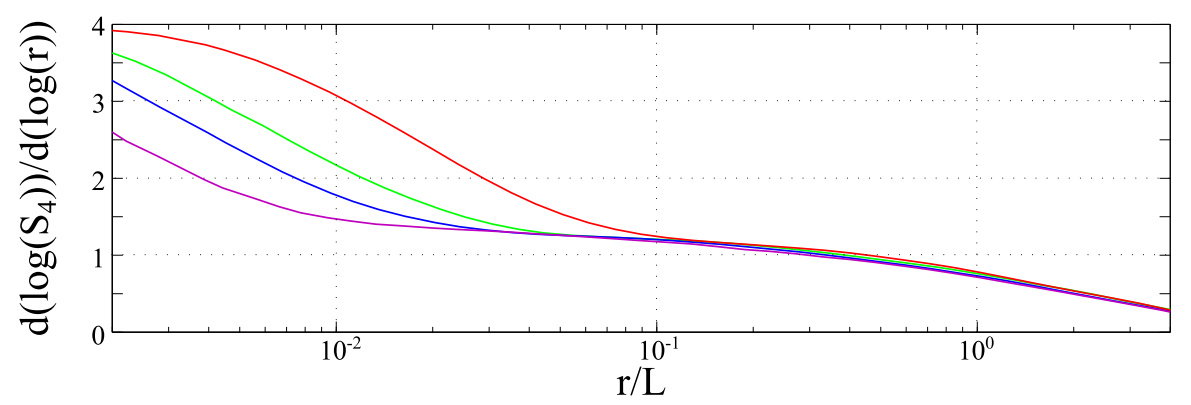

Figure 4.4: Logarithmic derivative of the fourth-order structure functions measured with NSTAPs. The shown data and color-coding is identical to that in figure 4.3. The curves approach a dissipation-range scaling of 4 . A plateau of value $4 / 3$ in the inertial range would be expected in the K41 framework. As in figure 4.3 , there is no clear scaling of any exponent at any Reynolds number.

As with the logarithmic derivative of the third-order structure function, no such plateau or even approach towards a plateau can be observed. Identical observations can be made for any arbitrary structure function. The absence of scaling is thus not a feature limited to the third-order structure function but a general intrinsic feature of turbulence. In order to capture the deviation from the ideal scaling in a quantitative manner, it is useful to compute the derivative of the curves in figure 4.3 with regards to the separation, $\mathrm{d}^{2} \log \left(S_{3}\right) / \mathrm{d}(\log (r))^{2}$. This derivative 
gives local information about the slope of the logarithmic derivatives of the thirdorder structure function. For this quantity, a value of zero would correspond to a local power-law scaling with an arbitrary exponent, whereas non-zero values correspond to a drift in the scaling exponents with increasing Reynolds number. Figure 4.5 shows the minimal local deviation from a power-law scaling in the inertial range, defined as $\delta=\min \left|\left[\mathrm{d}^{2} \log \left(S_{3}\right) / \mathrm{d}(\log (r))^{2}\right]\right|$ versus the Reynolds number for all measurements in the datasets Statistic_Large and Statistic_Huge. Due to the limited amount of statistical information in the dataset Statistic_Medium, the results from this datasets are excluded, as an unbiased second derivative of the third-order structure functions carries too high statistical noise in the large scales to reliably extract information about the minimum deviation. The information from this dataset might be recovered using smoothing techniques, an approach not applied here in order to prevent any a-priori modeling influencing the results.

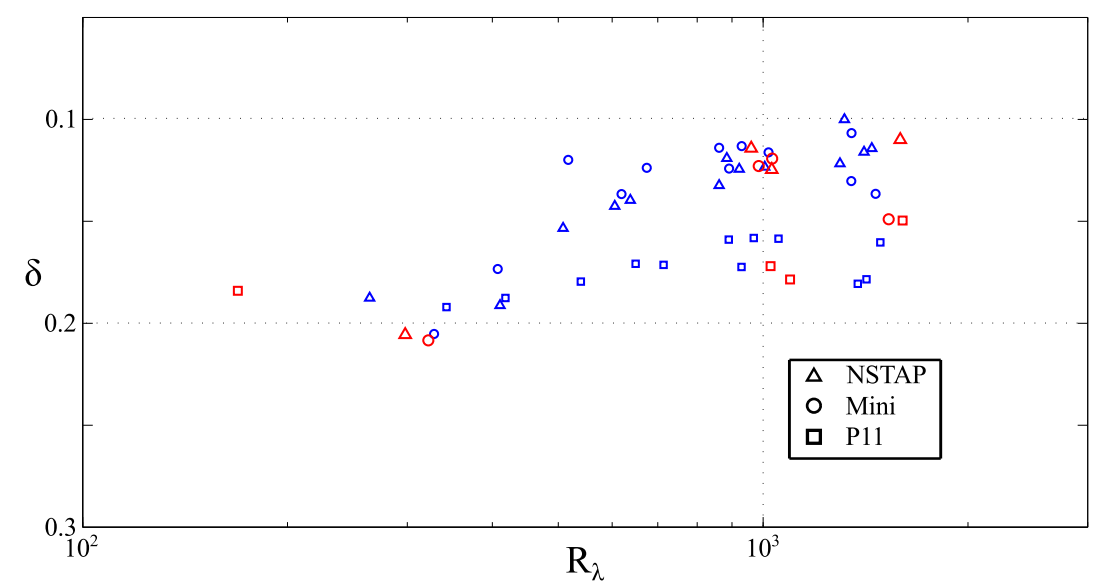

Figure 4.5: Deviation from local power-law scaling in the inertial range as a function of the Reynolds number. Blue symbols correspond to measurements in the dataset Statistic_Large and red symbols to the dataset Statistic_Huge. 
The minimal deviations $\delta$ can be fitted with a power-law as $\delta=0.67 \cdot R_{\lambda}^{-0.23}$. Assuming that this trend continues without and with no transition to a new regime at higher Reynolds numbers, this extremely slow algebraic approach to ideal scaling means that there are no natural turbulent flows which obey true scaling. Defining a still noticeable local deviation of $\delta=0.01$ as sufficiently small to speak of ideal scaling behavior, one would need a flow with an extrapolated Reynolds number of $R_{\lambda}>10^{8}$ to realize such a deviation. The deviations from ideal scaling for structure functions of other orders behave in a similar way. This finding is of importance in modeling extreme events in turbulence. As the scaling exponents are connected to the occurrence of extreme events, like, e.g., wind gusts, models using scaling properties of turbulent flows to predict these events might over- or under-predict their rate. The occurrence of these extreme events does play, e.g., an important role in the design of wind turbines.

\subsection{Extended Self-Similarity}

Apparently, the structure functions do not exhibit scaling in the sense of Kolmogorov's predictions. Empirically, however, it has been found by Benzi et al. (1993) that it is possible to identify scaling-like behavior in the $\mathrm{n}^{\text {th }}$-order structure function $S_{n}$, if one does not search for scaling with respect to the separation, but rather with respect to structure functions of different order. This approach is known as Extended Self-Similarity (ESS). The proposal was that there might be scaling in the sense of the structure functions themselves according to 


$$
S_{n} \sim S_{m}^{\zeta_{n, m}}
$$

Here, $\zeta_{n, m}$ is a relative scaling exponent, connecting the structure functions of orders $n$ and $m$. It turns out that choosing the aforementioned third-order structure function of the absolute values, $S_{3 a}=\left\langle|\delta u|^{3}\right\rangle$, to be $S_{m}$ in the above equation provides the best results for unveiling scaling properties. Additionally, even-order structure functions seem to follow this proposed relationship more closely than odd-order ones. In the original publication, the authors chose to plot the $\mathrm{n}^{\text {th }}$-order structure function against the third-order structure function of the absolute values. From power-law fits to this relation, they obtained information about the scaling properties. If one assumes $\zeta_{3 a}=1$, one can relate the scaling exponents in the sense of ESS with the classically defined scaling exponents. As a consequence, one can formulate ESS by other means. If scaling in the sense of Extended Self-Similarity holds true, the structure functions of $n^{\text {th }}$-order scale as generalized power-laws of the form

$$
S_{n} \sim(f(r) \cdot r)^{\zeta_{n}}
$$

ESS claims that the unknown function $f(r)$ is independent of the order and thus drops out when investigating the relation between structure functions of different order. The approach of plotting structure function of different order against each 
other in order to extract relative scaling exponents is feasible. However, a more direct way of investigating the relation is again to compute the logarithmic derivatives of equation (4.9) with

$$
\frac{\mathrm{d} \log S_{n}}{\mathrm{~d} \log S_{m}}=\zeta_{n, m}
$$

The latter approach was chosen in figure 4.6. This figure compares the direct scaling as in figure 4.4 with the scaling in the sense of ESS.

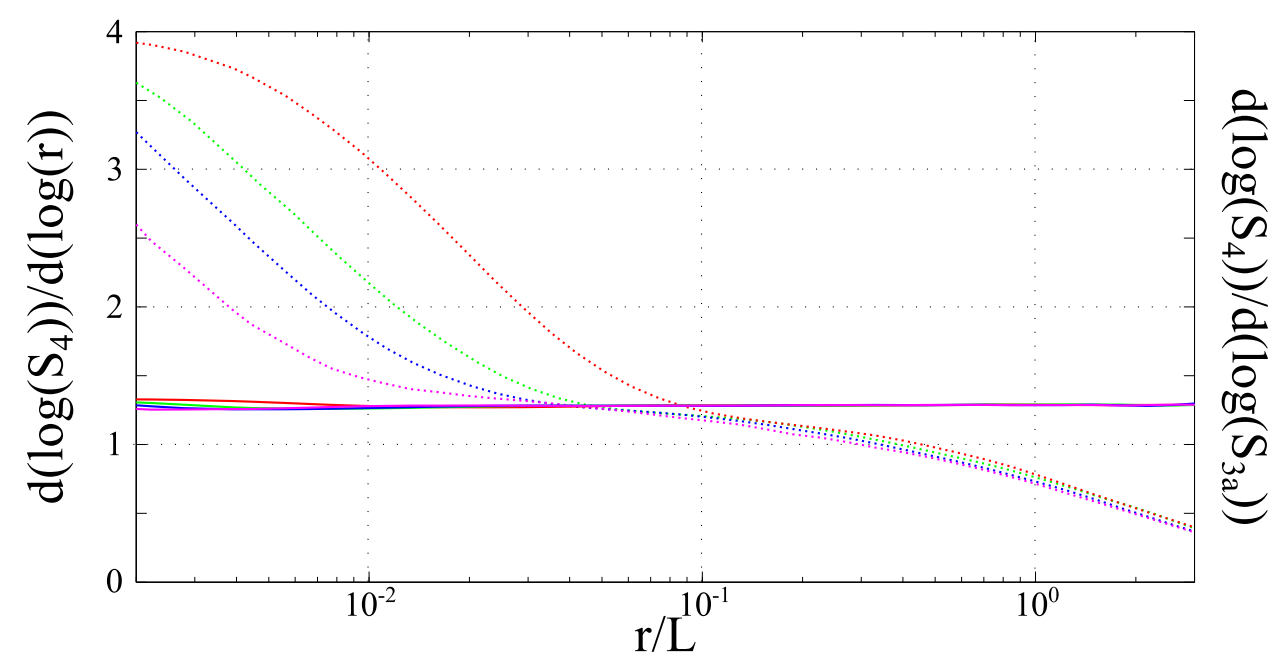

Figure 4.6: Logarithmic derivative of the fourth-order structure function with regards to the separation $r$ (dashed lines) and to the third-order structure function of the absolute value $S_{3 a}$ (solid lines). The data shown is identical to that in figure 4.4. The Reynolds number of the measurements are $R_{\lambda}=300$ (red) $R_{\lambda}=610$ (green), $R_{\lambda}=860$ (blue) and $R_{\lambda}=1320$ (purple). Applying Extended Self-Similarity greatly improves the scaling properties of the structure function. 
A direct comparison yields favorable results for Extended Self-Similarity at all Reynolds number over a wide range of scales. The deviations from strict scaling are significantly lower, using the empirical methods of ESS. The standard procedure, once ESS has been applied, would be to directly compute the scaling exponents from figure 4.6 and continue with a literature comparison. However, a detailed view on the ESS curves uncovers limitations of the applicability of this method in its very basics. Figure 4.7 shows the logarithmic derivatives of the fourth-order structure function by the third-order structure function of the absolute value; figure 4.8 of the sixth-order by the second-order, respectively. The data shown is identically to that in figure 4.6, thus spanning Reynolds numbers between $\operatorname{Re}_{\lambda}=300$ and $\operatorname{Re}_{\lambda}=1320$.

Several distinct features can be observed, regardless of Reynolds number. At the small scales, between dissipation range and the intermediate scales, the curves show an oscillatory transition with a distinct mininum. This near-dissipation range effect was already observed by, e.g., Frisch \& Vergassola (1993), Herwijer \& van de Water (1995) and Chevillard et al. (2005) along the lines of the multifractal models. However, despite popular belief, from figures 4.7 and 4.8 it becomes apparent that dissipative effects do not only influence the statistics up to $20 \eta$ but significantly into the intermediate range of scales. At about $110 \eta$, a secondary oscillatory maximum emerges independently of Reynolds number and order of the investigated structure functions. At scales larger than $100 \eta$, in contrast to the expectations from Extended Self-Similarity, there is no plateau emerging. Instead, even at the highest Reynolds numbers, there is a systematic positive slope. 

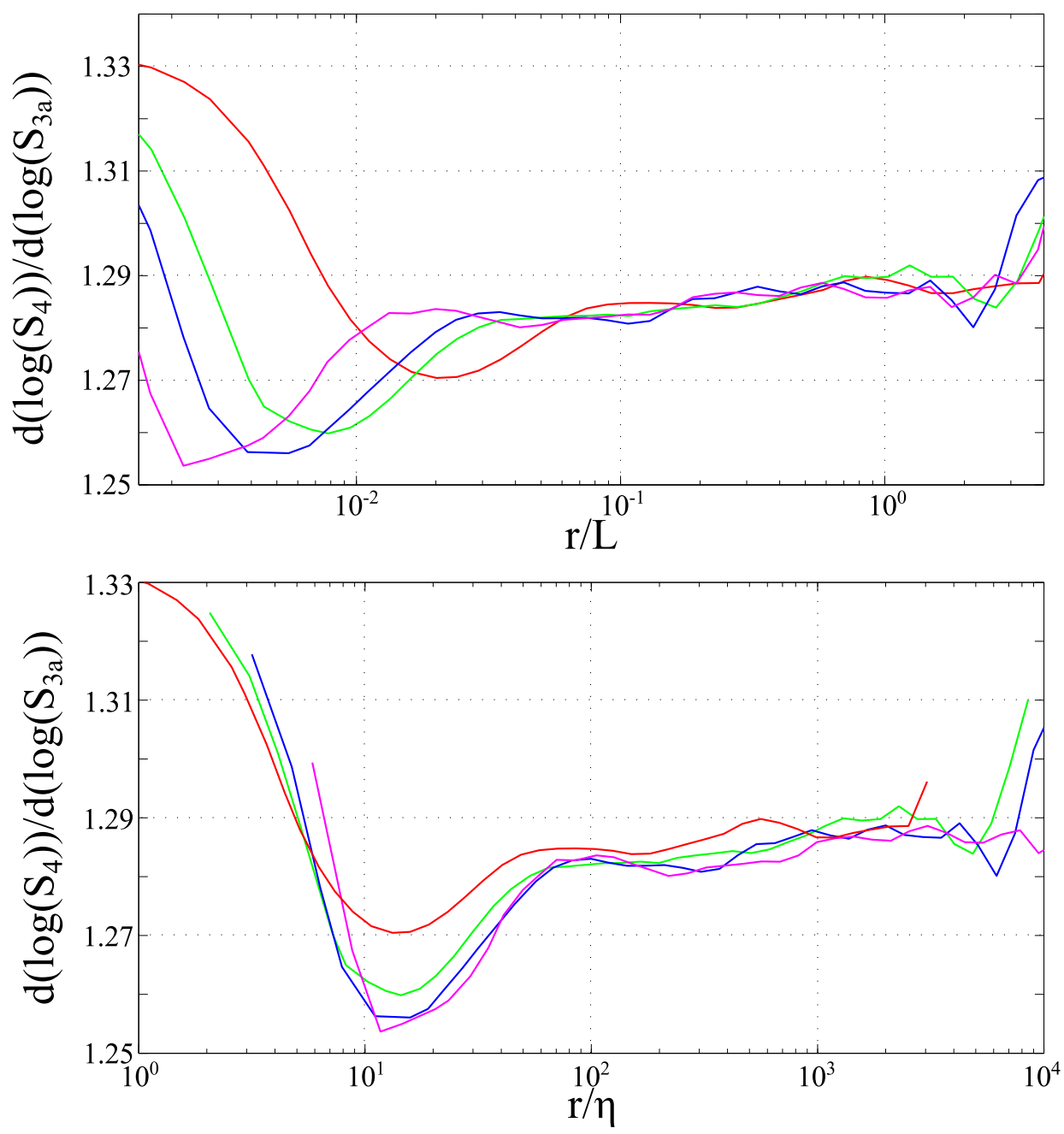

Figure 4.7: Detailed view on the scaling behavior in terms of extended selfsimilarity of the fourth-order structure function. Shown are the same curves as above, normalized by the large scales (top) and small scales (bottom). Regardless of Reynolds number, Extended Self-Similarity does not uncover scaling properties. Dissipative effects penetrate the inertial range up to roughly $100 \eta$. For $r>100 \eta$ there is a systematic slope indicating the absence of ideal scaling along the lines of Extended Self-Similarity. 

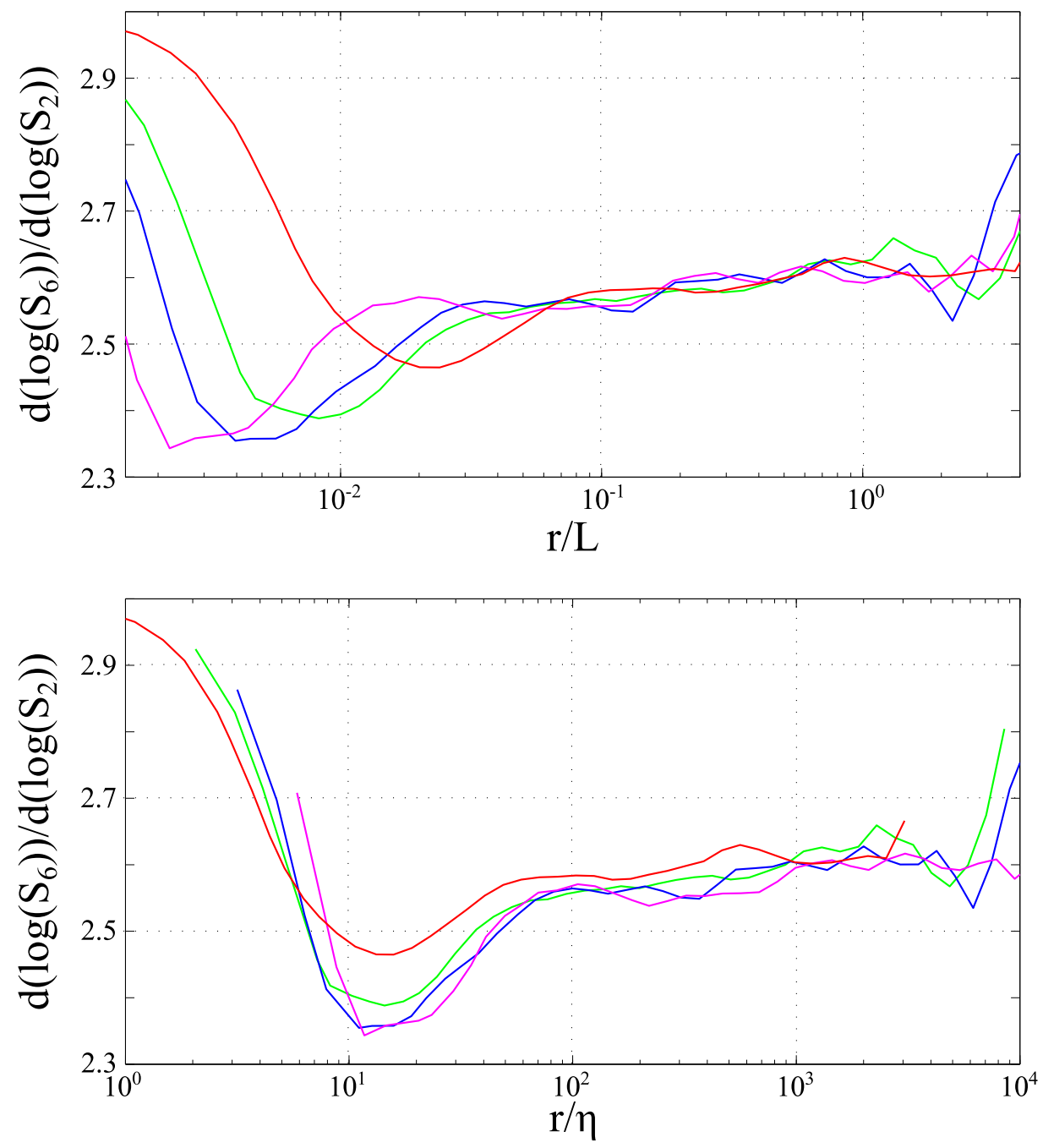

Figure 4.8: Analogous calculation to figure 4.7 by computing the logarithmic derivative of the sixth-order structure function with regards to the second-order structure function. The extent of dissipative effects and the absence of scaling is reproduced for any combination of structure functions, meaning that these feature are not unique to the third-order structure function of the absolute value but universal for turbulence. 
This feature only becomes visible due to the amount of recorded data in the datasets Statistic_Large and Statistic_Huge. For shorter datasets, the fine details of the inertial range behavior is succumbed by noise. The drift in the local slope indicates the absence of strict scaling in the sense of ESS, just as there was no strict scaling in the classical sense. As a consequence, neither the existence of a true inertial range can be observed even at the highest Reynolds numbers nor is there an unambiguous definition of scaling exponents at all.

The effect of dissipation on the intermediate scales can be understood by going back to Kolmogorov's equation (1.24). Usually, the viscosity-dependent term on the left-hand side of the equation is neglected in the inertial range and believed to not matter once the Reynolds number is high enough. However, several studies investigated the Reynolds-number effects on Kolmogorov's equation at $R_{\lambda}<100$, finding that dissipative effects only vanish slowly with increasing Reynolds number (Zhou \& Antonia 2000, Antonia \& Burattini 2006). There is also a line of thought that the general concept of an inertial range is ill-defined (Kholmyansky \& Tsinober 2009). Figure 4.9 shows the relative importance of dissipation as a function of scale in terms of the ratio of the viscous term of the second-order structure function to the third-order structure function for a wide range of high Reynolds numbers. The curves correspond to measurements at Reynolds numbers between $R_{\lambda}=110$ (cyan) and $R_{\lambda}=1450$ (red). Normalized by the Kolmogorov length scale, the curves collapse very well and can be approximated by a two-component power-law, $a \cdot(r / \eta)^{b}+c \cdot(r / \eta)^{d}$. At the smallest scales, the ratio follows the trivial dissipationrange scaling of $r^{-2}$. For scales larger than $26 r / \eta$, the ratio transitions into a shallow 
scaling with $r^{-1.2}$, independently of Reynolds number. This finding implies that the influence of dissipative effects is completely universal with regards to the Reynolds number and only decays slowly with scale. Independent of the Reynolds number, the statistics of turbulent flows are affected in a non-negligible way by dissipation at significantly large scales, resulting in the difficulty of observing a clear inertial range.

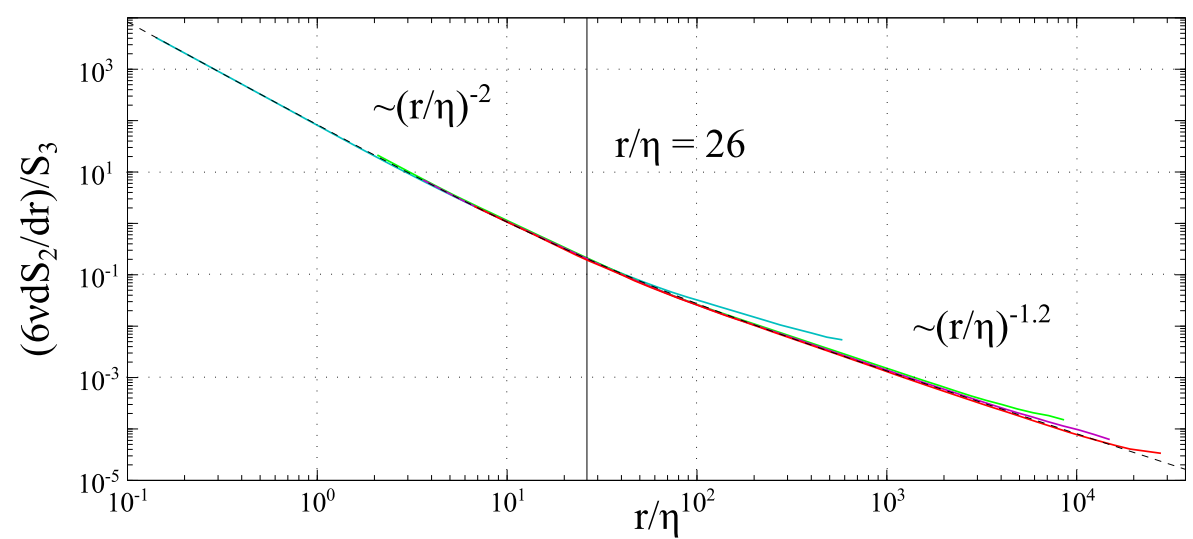

Figure 4.9: Relative importance of the dissipative term in Kolmogorov's equation in a log-log plot. The data shown is from NSTAP measurements from the dataset Statistic_Large at Reynolds numbers $R_{\lambda}=110$ (cyan), $R_{\lambda}=610$ (green), $R_{\lambda}=920$ (purple) and $R_{\lambda}=1450$ (red). The effect of dissipation is completely universal with regards to the small scales and decays algebraically slow with with scale. The deviations at the large scale end of the curves stem from statistical noise at those scales and limitations of isotropy due to the tunnel size. The dashed line is an empirical two-exponent power-law fit to the data, excluding the large scale deviations, of the form $a \cdot(r / \eta)^{b}+c \cdot(r / \eta)^{d}$. The horizontal black line denotes the length scale at which the contribution of both parts of the fitted power-law is equal and can be understood as a transition length scale between the dissipation range and the inertial range. 


\subsection{Effective Scaling Exponents}

Even though ESS was shown to fail to uncover scaling in turbulence, it is useful do define 'effective' scaling exponents to comment on the previous findings in the literature and investigate Reynolds number influences. As shown earlier, an unambiguous definition of a scaling exponent is impossible by the absence of real scaling. One way to define an effective scaling exponent is to find a range of scales over which the local slope is averaged. The resulting scaling exponent will obviously depend on the choice of the interval, as there is no plateau corresponding to strict scaling. In this work, the lower bound of the averaging interval is chosen to be at a scale two times larger than the scale of the secondary maximum near the dissipation range and the large scale limit is fixed at the integral length scale with $r / L=1$. To estimate up to which order of exponent a definition is still meaningful, it is mandatory to check for statistical significance in terms of the cumulants of the velocity increment probability density functions. For a certain scaling exponent to have physical meaning, the respective cumulant of the velocity increment probability density function needs to be still resolved. Figure 4.10 shows the cumulants up to order 16 of the probability density functions of velocity increments for a fixed separation of $100 \eta$. While for small cumulants, it does not matter whether 8 or 56 hours long datasets are used to compute the exponents, for higher cumulants, the short datasets lose a significant part of shape information due to noise. This demands for the large amount of statistics in the dataset Statistic_Huge to extract meaningful high-order scaling exponents. 

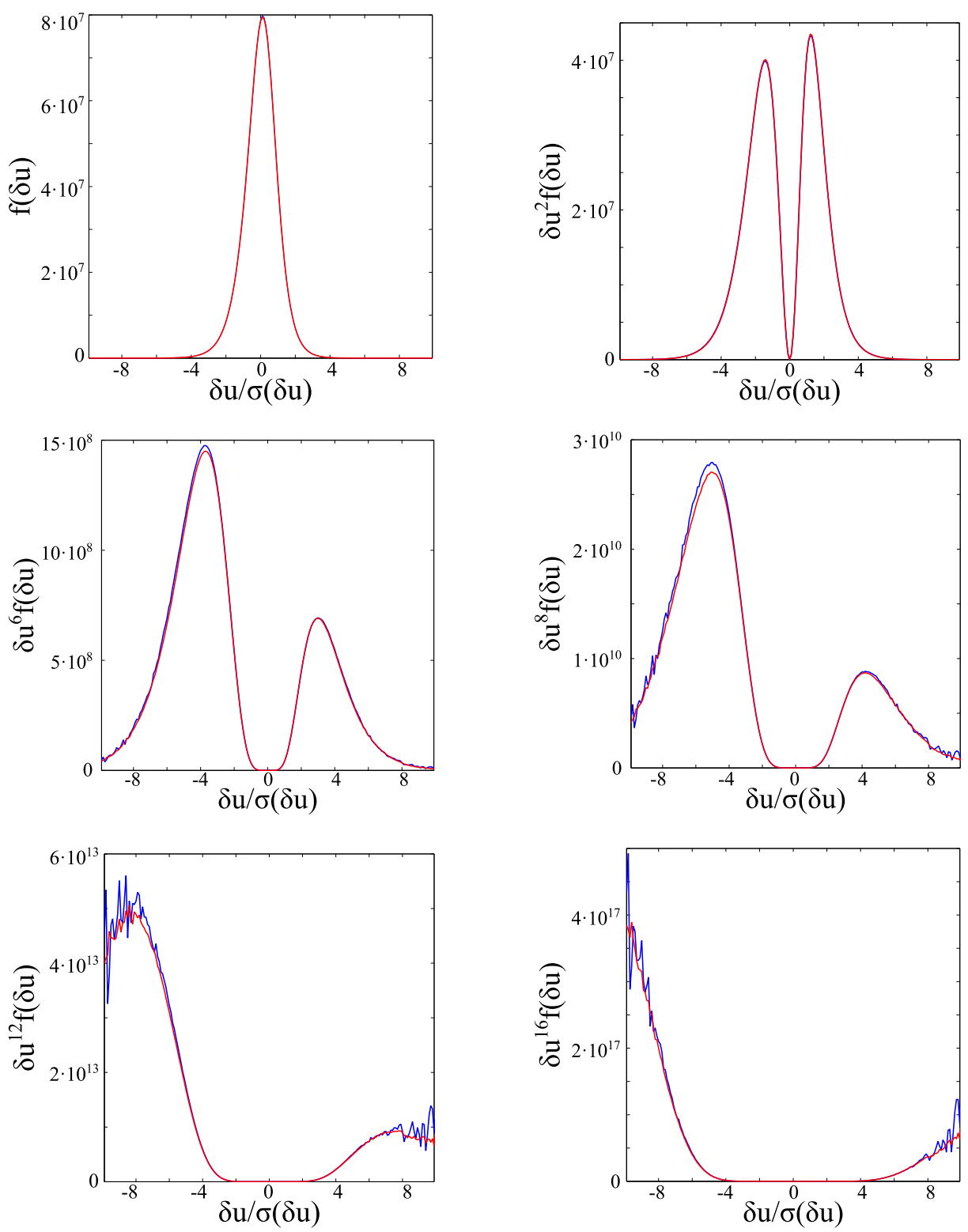

Figure 4.10: Non-normalized cumulants of the probability density functions of velocity increments with a separation of $100 \eta$. The dataset shown was measured with an NSTAP at $R_{\lambda}=1600$ between -10 and 10 standard deviations of the velocity increments, $\sigma(\delta u)$. The blue curves correspond to 8 hours, the red curves to 56 hours long times series. The differences in the quality of the statistics is negligible for low moments of the probability density functions, but becomes significant at higher orders. 
Figure 4.11 shows the ratio of the so-defined effective scaling exponents to their expected K41 values. For high Reynolds numbers, the effective scaling exponents assume a constant value, making it possible to compute precise values under the assumption $\zeta_{3 a}=1$. For the low order structure functions, the thresholding effect has been observed by Antonia et al. (2000). However, the VDTT results offer an extended interpretation. The Reynolds number only influences the behavior of the effective scaling exponents as long as they have not reached an order-dependent threshold. Low-order effective scaling exponents become constant at much lower Reynolds numbers than higher-order exponents. Thus, it is not only necessary to measure extremely long datasets to extract meaningful effective scaling exponents, it is also necessary to conduct the experiments at high Reynolds numbers. Averaging over all those individual results of an effective scaling exponents that are in the Reynolds number independent region, the results from figure 4.11 yield $\zeta_{2}=$ $0.6911 \pm 0.0001, \zeta_{4}=1.2845 \pm 0.0002, \zeta_{6}=1.7827 \pm 0.0009, \zeta_{8}=2.190 \pm 0.002$, $\zeta_{10}=2.509 \pm 0.005$ and $\zeta_{12}=2.74 \pm 0.02$. The errors given here a purely statistical standard errors of the mean. For a more detailed analysis, see section 4.5.

In order to compare the data with previous results, it is necessary to define relative scaling exponents $\zeta_{m, n}$ as $\zeta_{m}-\zeta_{n}$. This has to be done since the theoretical models enforce $\zeta_{3}=1$ and most experimental studies assume $\zeta_{3 a}=1$. This is not in agreement with the findings presented in section 4.2. By investigating relative scaling exponents, any dependence on the value of the third-order scaling exponent cancels out. 


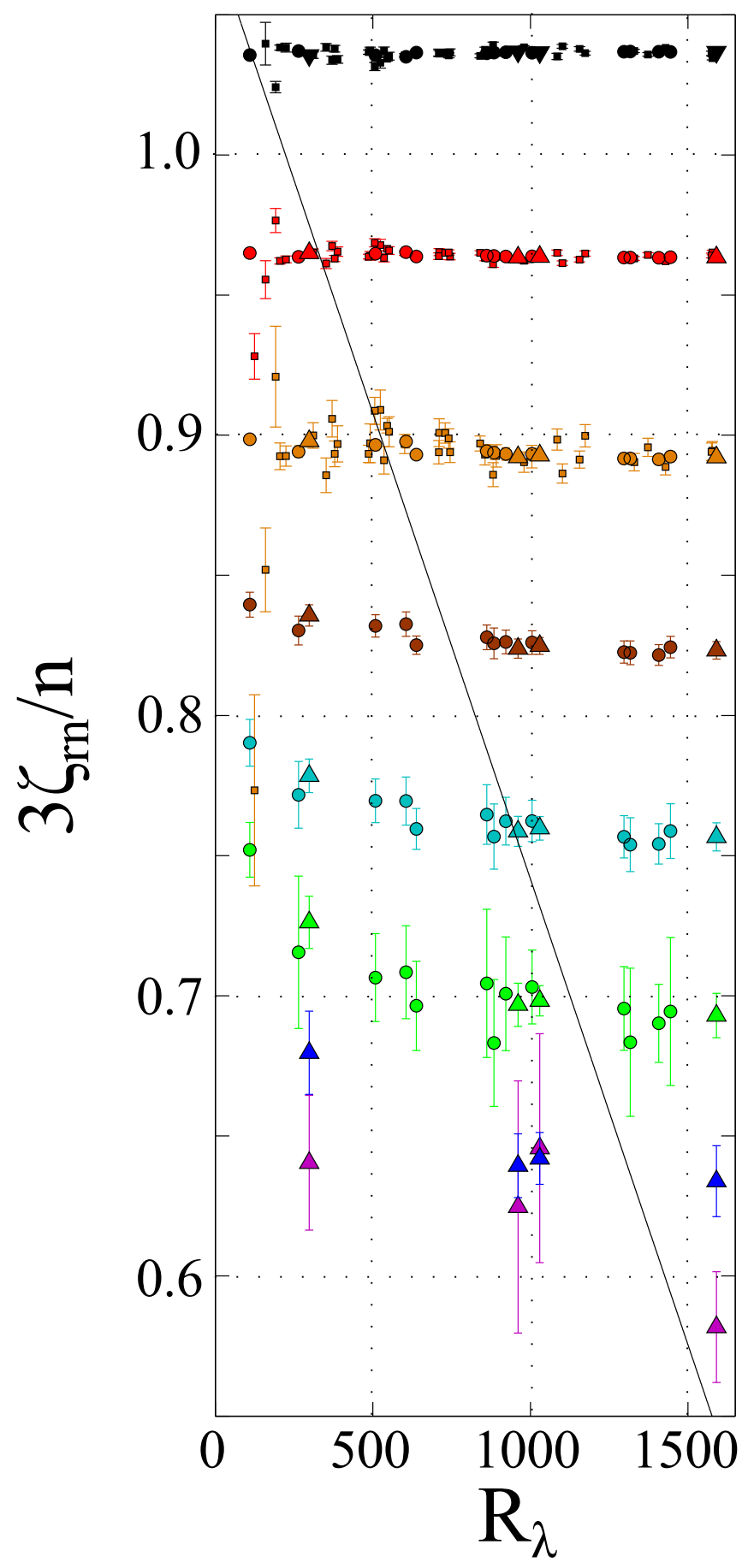

Figure 4.11: Deviation of the obtained even-order relative scaling exponents $\zeta_{r n}$ to the value $n / 3$ predicted by K41 between $n=2$ (black) and $n=16$ (purple), assuming $\zeta_{3}=\zeta_{3 a}$. The scaling exponents are completely Reynolds number independent once a threshold, depicted by an empirical black line is reached. Triangles correspond to the dataset Statistic_Huge, circles to the dataset Statistic_Large and squares to the dataset Statistic_Medium. The error bars are computed as the standard error of the mean, assuming uncorrelated local measurements of the exponents. 
Figure 4.12 and tables 6.6 to 6.8 in the Appendix give a comparison of the measured effective relative scaling exponents with results from earlier experiments, direct numerical simulations and theoretical models. In the theoretical models, all occurring parameters were assumed to be free and were fitted to the VDTT data to obtain the most favorable agreement. No physical restriction has been applied to these parameters. There has been an extensive amount of effort over the previous decades to precisely and accurately measure and predict scaling exponents. Especially the question, which model accurately describes the statistical behavior of turbulence in the inertial range, is of significant interest. For example, a working model for scaling exponents could be used as a tool to predict the rate of extreme events in connection to the higher-order exponents. Neither experiments nor numerical simulations were able to prove or disproof the viability of the more sophisticated models. While this has been seen as a lack of quality of data by either too short statistics or too low Reynolds numbers, the results from this chapter support a different interpretation.

In figure 4.12, the black error bars denote the systematic error in the definition of the scaling exponents and give a measure on how ill-defined a scaling exponent of that order is due to the absence of strict scaling. In the following, these will be referred to as boundaries of ill-definition. For an exact definition see section 4.5. The predictions of the older scaling models by Kolmogorov (1941b), Frisch et al. (1978), Benzi et al. (1984) and Meneveau \& Sreenivasan (1987a) do not lie within the boundaries of ill-definition of the exponents measured in the VDTT. Thus, these models can be ruled out as working predictions for scaling exponents. 
However, the differences in the predictions of the more recent models by Kida (1991), She \& Lévêque (1994) and Dubrulle (1994) are significantly smaller than the ambiguity in the definition of an effective scaling exponents due to the inherent absence of scaling. It is, thus, impossible to distinguish between these individual models.

The resulting exponents from numerical simulations by Cao et al. (1996), Boratav \& Pelz (1997), Toschi et al. (1999) and Gotoh (2013) lie within the boundaries of ill-definition. In contrast, the experimental results from the literature scatter significantly more and partly exceed the boundaries of ill-definition. This can be partly attributed to the effect of low Reynolds numbers as seen in figure 4.11 and problems of statistical convergence. Notably two of experimental studies yield scaling exponents that differ substantially from the results obtained in the VDTT. The experiments by Belin et al. (1996) were conducted in liquid Helium, imposing challenges for a direct comparison. The underlying physical effects will have to be studied in greater detail to ensure that a direct comparability of the results is possible. van de Water \& Herweijer (1999) used a stretched exponential method to predict the shape of the tails of the probability density functions. These results are thus not directly data-driven but rather data-supported models. It is a priori unclear whether the deviations in the results stem from the data or from the chosen analysis.

To find a deeper understanding of the statistics and physics in the inertial range, more sophisticated models, not relying on the historically grown but non-existing properties of scaling are needed. 


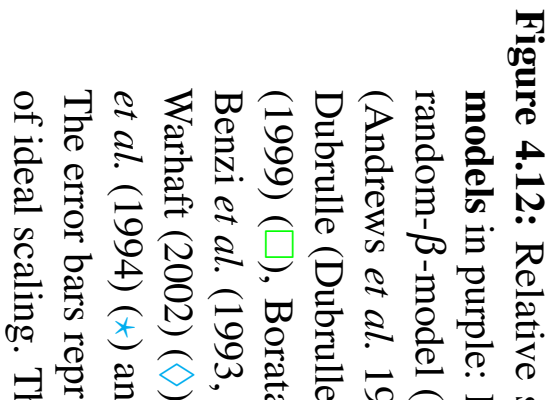

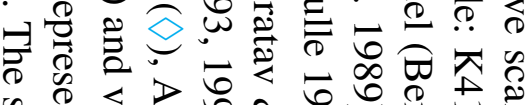

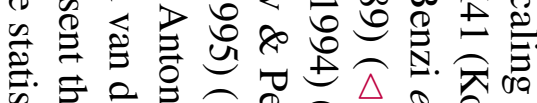

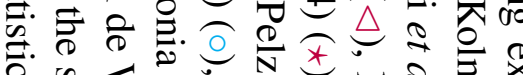

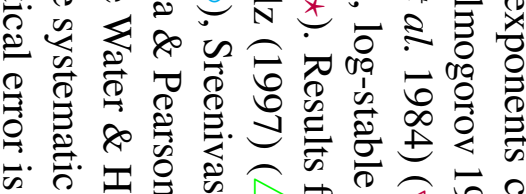

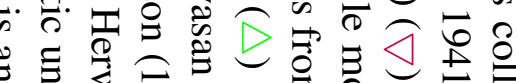

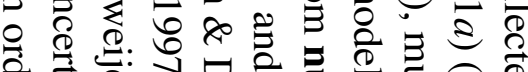

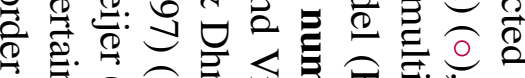

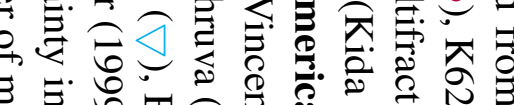
క

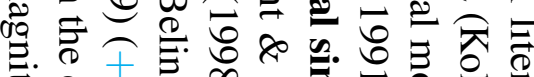

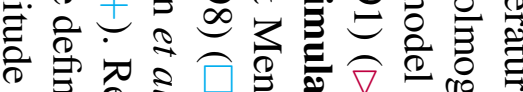

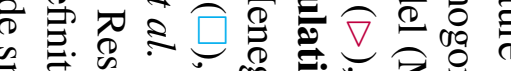

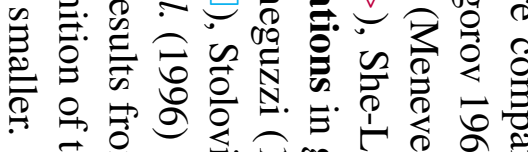

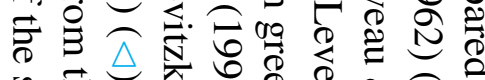

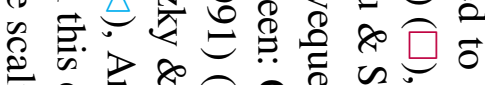

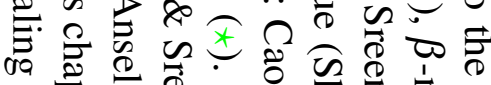

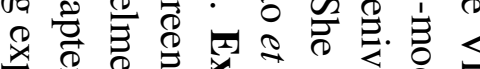

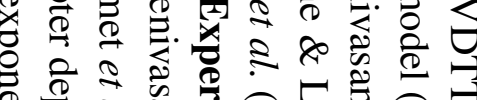
के

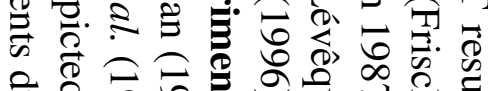

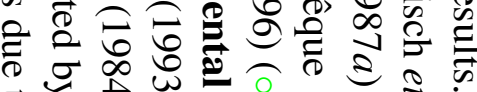
$\checkmark$ 过

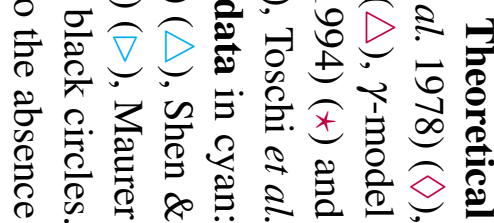
$\zeta_{n, 2}$
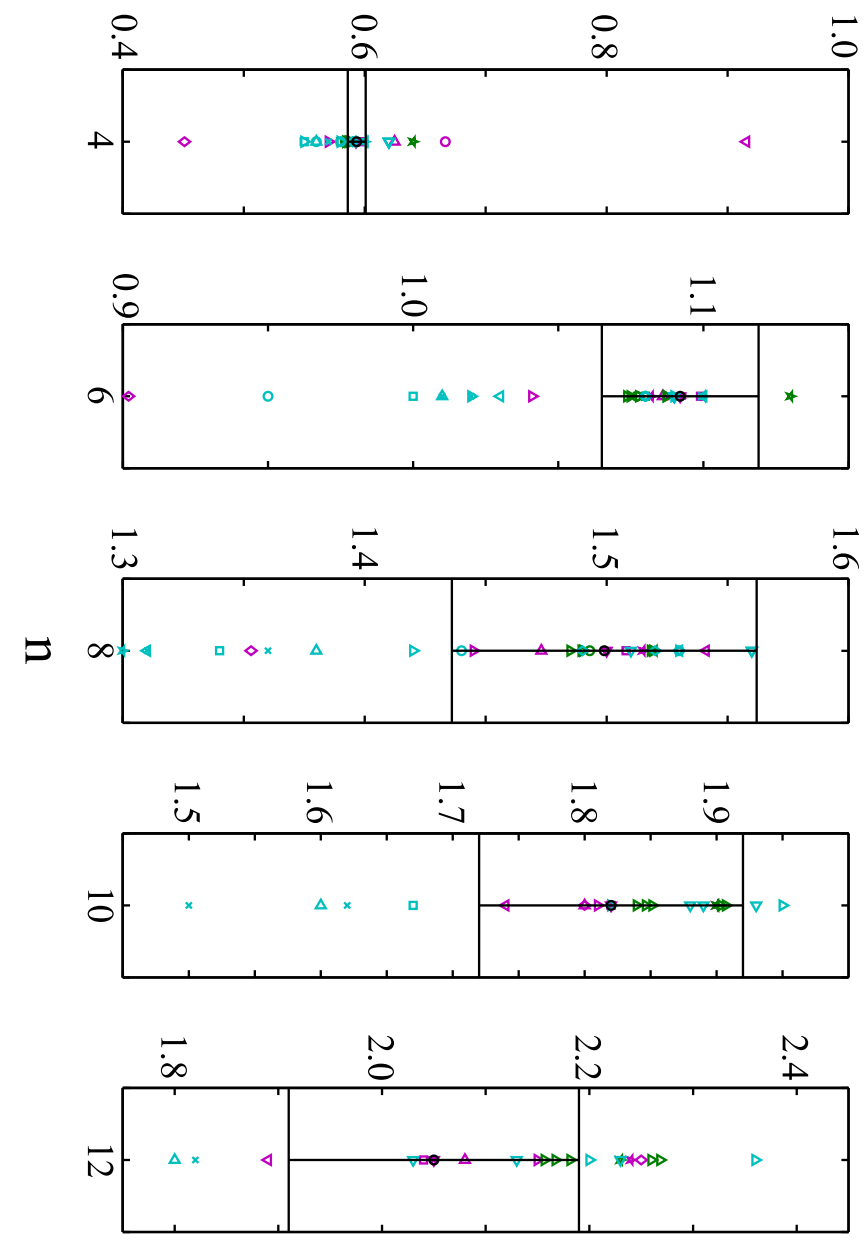


\subsection{Measurement Uncertainties}

Two major contributions of uncertainty affect the estimation of the effective scaling exponents: The statistical noise in the structure functions, due to under-resolved probability density functions, as shown in figure 4.10, and the principal inability to define scaling exponents, due to the lack of scaling. The error bars shown in figure 4.11 and the values given in the corresponding text represent the statistical uncertainties. Each individual measurement at a Reynolds number is influenced by statistical noise, resulting in scattered individual effective scaling exponents over which a global average is conducted. At a given Reynolds number $R_{\lambda i}$ and for a given probe, one obtains one scaling exponent $\zeta\left(R_{\lambda i}\right)$. If one assumes that every of the $N$ data points in the averaging range is a independent measurement, $\zeta_{j}$, of the scaling exponent with a random error, one can calculate the mean according to

$$
\zeta\left(R_{\lambda i}\right)=\frac{1}{N} \sum_{j=1}^{N} \zeta_{j}
$$

If one further assumes that the individual errors are normal distributed, the uncertainty $u_{\zeta\left(R_{\lambda i}\right)}$ of the mean can be calculated as

$$
u_{\zeta\left(R_{\lambda i}\right)}=\sqrt{\frac{1}{N(N-1)} \sum_{j=1}^{N}\left(\zeta_{j}-\zeta\left(R_{\lambda i}\right)\right)^{2}} .
$$

These are the error bars shown in figure 4.11. The assumptions of independent 
measurements and normal distributed errors are most certainly not fulfilled, but rather give a computable estimate of the statistical error. To compute the globally averaged Reynolds number-independent effective scaling exponent of $\mathrm{n}^{\text {th }}$-order, $\zeta_{n}$, the $M$ individual results $\zeta\left(R_{\lambda i}\right)$ are averaged, so that

$$
\zeta=\frac{1}{M T} \sum_{i=1}^{M} t_{j} \cdot \zeta\left(R_{\lambda i}\right)
$$

where $t_{j}$ is the length of the datasets and $T=\sum_{j=1}^{M} t_{j}$. Each of the individual $\zeta\left(R_{\lambda i}\right)$ has its uncertainty calculated as above. So the uncertainty $u_{\zeta}$ of the mean $\zeta$ is

$$
\begin{aligned}
u_{\zeta} & =\sqrt{\left(\frac{\partial \zeta}{\partial \zeta_{1}} \cdot u_{\zeta_{1}}\right)+\left(\frac{\partial \zeta}{\partial \zeta_{2}} \cdot \zeta_{2}\right)+\ldots} \\
& =\sqrt{\left(\frac{t_{1}}{M T} u_{\zeta_{1}}\right)^{2}+\left(\frac{t_{2}}{M T} u_{\zeta_{2}}\right)^{2}+\ldots} \\
& =\frac{1}{M T} \sqrt{\sum_{i=1}^{M}\left(t_{i} u_{\zeta_{i}}\right)^{2}} .
\end{aligned}
$$

These are the errors given in the text and in table B in Appendix B. As shown in section 4.4 , however, the definition of the scaling exponent itself is problematic, as there is no strict scaling in the sense of ESS. The logarithmic derivatives of the structure functions with respect to the structure functions of different order show a systematic drift over the intermediate scales. This leads to an ill-defined effective scaling exponent which depends on the magnitude of the drift along the 
averaging interval. To quantify the extent of ill-definition, the systematic error in the determination of the effective scaling exponents can be defined in the following way. For every individual measured logarithmic derivative of the structure functions, a linear fit in logarithmic coordinates is applied in the averaging region. The change of value of the fitted line along the averaging interval gives an estimation of the systematic error in determination of the effective scaling exponents. For this chapter, the systematic error was taken to be half of the change of value of the fitted line. Figure 4.13 gives an example of the definition of the systematic error for a NSTAP measurement from the dataset Statistic_Huge at $R_{\lambda}=1600$. In contrast to the statistical error, which can be reduced by measuring longer datasets, this systematic error is due to the underlying physics of turbulence and the absence of scaling. The average over all individual systematic uncertainty was used in figure 4.12 as error bars to emphasize the general inability to distinguish between models assuming scaling properties, since the arbitrariness in the definition of the scaling exponents is bigger than the differences in most of the model predictions.

\subsubsection{Probe Size Effects}

Up to this point, only data obtained with NSTAP probes has been considered. The reason for this is that, even though the measurements of the effective scaling exponents are measurements of the intermediate length scales, the size of the hot-wire does become important at higher Reynolds numbers. In figure 4.11, the effective scaling exponents reached a constant value with Reynolds number once an order-dependent threshold was reached. Figure 4.14 shows the effective scaling 


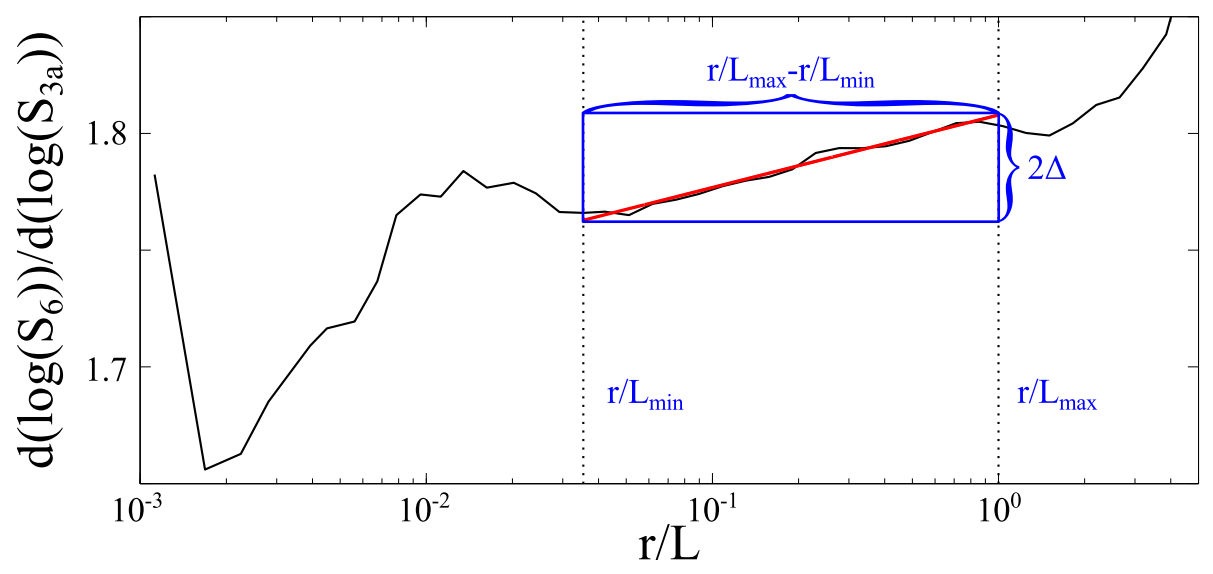

Figure 4.13: Systematic uncertainty due to the absence of scaling in the sense of ESS. Shown is the logarithmic derivative of the sixth-order structure function with respect to the third-order structure function of the absolute value in a semi-logarithmic plot from NSTAP data at $R_{\lambda}=1600$. The averaging interval to determine the effective scaling exponent is denoted with dashed lines. The red line shows a fit to the data (black). The altitude of the fitted line is taken as a definition for twice the systematic error on the scaling exponents, $\Delta$. This uncertainty is an order of magnitude larger than the statistical uncertainty.

exponents for all three probe types: NSTAP, Mini and P11. While the NSTAP data shows the aforementioned plateau, Mini and, even more so, P11 probes show significant deviation from this behavior. While for low Reynolds numbers, the measured exponents of the three probes agree within the uncertainties, deviations become apparent at high Reynolds numbers. Once the relevant scales become small, the size of the probes becomes relevant, too, since important information is spatially averaged. Using Mini or P11 probes to investigate the Reynolds number dependence of the scaling exponents will lead to the spurious conclusion that for higher Reynolds number, the deviations from K41 scaling becomes smaller. This finding, however, only arises from the lack of spatial resolution at higher Reynolds 
numbers. This finding complicates the estimation of high-order effective scaling exponents regardless of the systematic ill-definition. While figure 4.11 implies that the measurement has to be conducted at as high Reynolds numbers as possible, the results from figure 4.14 limit the investigable range of Reynolds numbers by the size of the measurement device. Even with arbitrarily long time series, these findings limit the maximal obtainable order of effective scaling exponents by Reynolds number and probe size. 


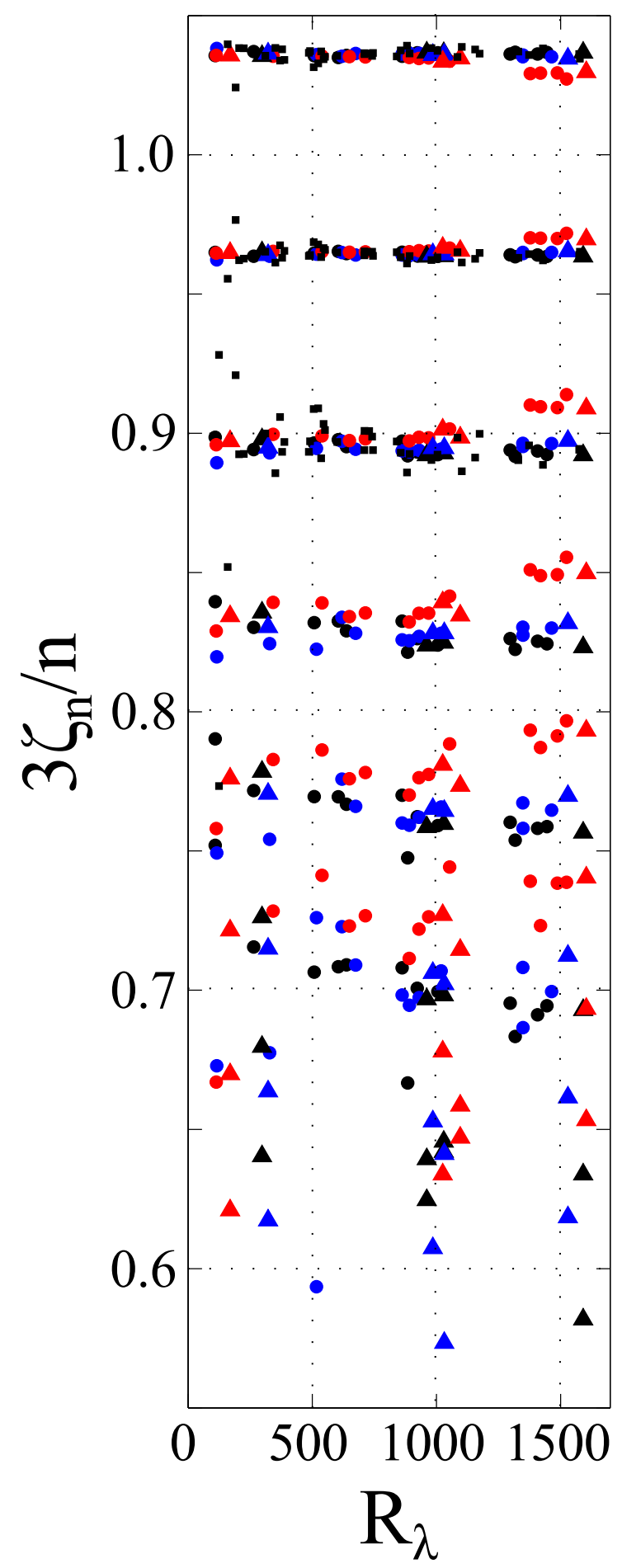

Figure 4.14: Effect of the size of the measurement probes on the effective scaling exponents. Black symbols correspond to NSTAP data, blue symbols to Mini data and red symbols to P11 data. Triangles correspond to the dataset Statistic_Huge, circles to the dataset Statistic_Large and squares to the dataset Statistic_Medium. The NSTAP data is identical to that in figure 4.11. While the NSTAPobtained exponents reach a constant value with high Reynolds numbers, Mini and, even more so, P11 data exhibits a spurious Reynolds number dependence, due to limitations of the probe size. 


\section{Statistics of the Small Scales}

In this chapter, I investigate the statistics of the small scales of turbulent flows, using data acquired in the S1MA at Onera in Modane, France, in the scope of the ESWIRP project "Investigation of the small-scale statistics of turbulence in S1MA". Section 5.1 gives an overview over the flow properties in the wind tunnel. The statistics of the velocity derivatives are discussed in section 5.2 and in section 5.3, I discuss the build-up of intermittency towards the smallest scales in the turbulent flow.

\subsection{Flow Properties}

As discussed in section 2.2, the S1MA is a gigantic wind tunnel with an outer length of $155 \mathrm{~m}$, a width of $40 \mathrm{~m}$ and a diameter of the test section of $8 \mathrm{~m}$. Turbulence is produced by an inflatable grid with a mesh spacing of $0.6 \mathrm{~m}$ and round grid bars. The mean wind speed for the two experiments analyzed in this chapter was $21 \mathrm{~m} / \mathrm{s}$ and $43 \mathrm{~m} / \mathrm{s}$, respectively. As the wind tunnel uses atmospheric air as a working gas and does not have a heat exchanger comparable to the one in the VDTT, the mean temperature in the tunnel depends on the ambient temperature. As discussed in 
subsection 2.3.2, the response of an NSTAP in a CTA circuit crucially depends on the temperature of the working gas. The non-constancy of the outside temperature leads to a drift in the mean signal of the NSTAP used to measure the turbulent time series in the S1MA. An additional temperature effect arose from the experimental setup. As the CTA system, including the external resistor decade, was placed outside of the test section in a non-temperature-controlled environment, changes in the mean temperature of the working gas did not equivalently translate into changes of the temperature of the CTA system. As a consequence, the temperature of the external resistor decade and the mean temperature of the working gas were decoupled, thus resulting in a non-constant overheat ratio. This effect contributes to the measured variation mean wind speed. Figure 5.1 shows the measured mean speed, averaged over $15 \mathrm{~s}$ intervals, for an experiment of the dataset S1MA (black). There is a significant downwards drift in the signal of about $0.7 \mathrm{~m} / \mathrm{s}$ over the course of just over one hour of measurement time.

This drift, however, can be approximated by a quadratic fit (red curve). As the actual mean speed of the tunnel was kept constant throughout the experiment, the fitted curve was used to remove the drift in the NSTAP signal by division of the signal with respect to the fitted curve. The resulting time series of averaged velocities is shown in blue. For all further analysis, the data in the dataset S1MA was detrended in this manner to remove spurious information from the time series.

The use of a grid with round grid bars results in turbulence intensities significantly lower, compared to the use of a grid with rectangular grid bars of the same dimensions (see section 3.3). As a consequence, the measured turbulence intensities in 


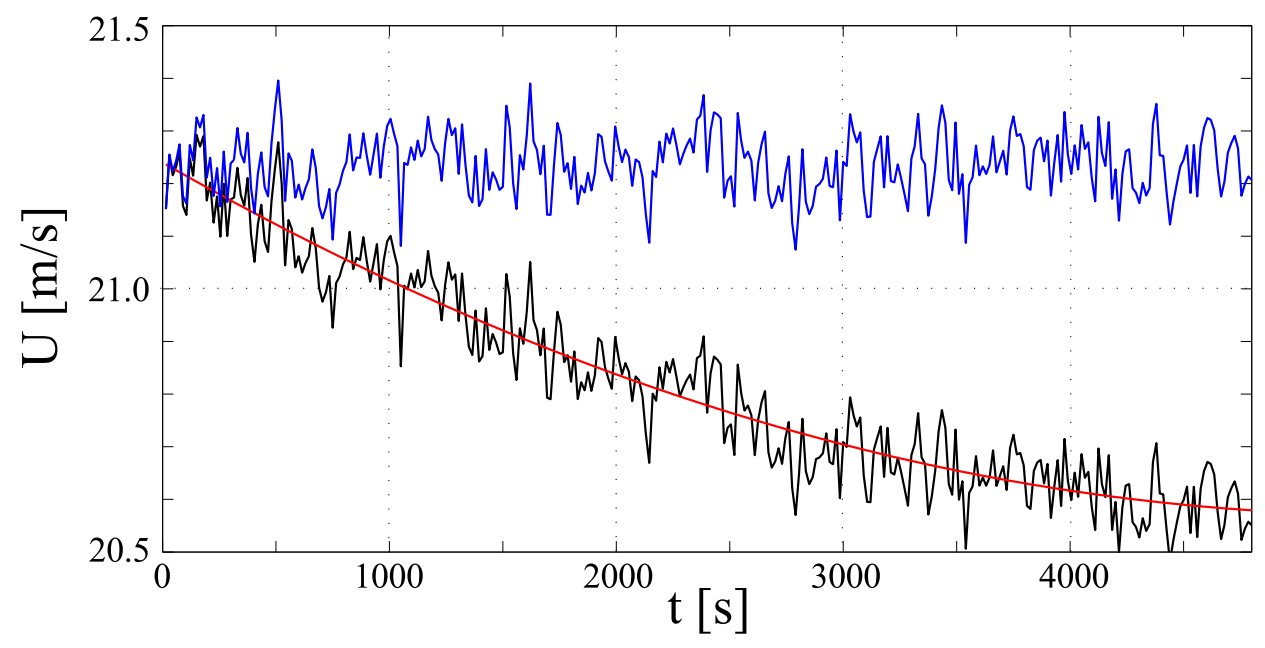

Figure 5.1: Mean velocity averaged over $15 \mathrm{~s}$ intervals as a function of measurement time. The original data (black) shows a significant trend due to changes in the mean temperature. The red curve is a fit to the data to detrend the signal (blue).

the S1MA, $u^{\prime} / U$, only reached values between $1.2 \%$ and $1.7 \%$ and thus were about a factor of 2 lower than the turbulence intensities in the VDTT. The Taylor-scale Reynolds numbers in the S1MA experiments were between 250 and 320, along with Kolmogorov scales between $260 \mu \mathrm{m}$ and $330 \mu \mathrm{m}$. The use of a $30 \mu \mathrm{m}$ NSTAP ensured that there were no spatial filtering effects on the velocity signal. Temporal filtering, due to increasing electric noise, occurred at scales $r_{c u t}=U /\left(2 \pi f_{\text {cut }}\right)$. Here, $f_{c u t}$ is the cut-off frequency due to high-frequency noise in the energy spectrum. For the measurements in the dataset $\mathbf{S 1 M A}$, the cut-off scale was about $1.3 \eta$, yielding unfiltered access to the major part of the dissipation range. As a comparison, the cut-off scale in the VDTT in the datasets Statisic_Large, due to a combination of temporal and spatial filtering, was between $\eta$ and $6 \eta$ at Reynolds numbers above 300. The data in the dataset S1MA, thus, provides a comparable, if not even slightly 
improved, access to the dissipation range.

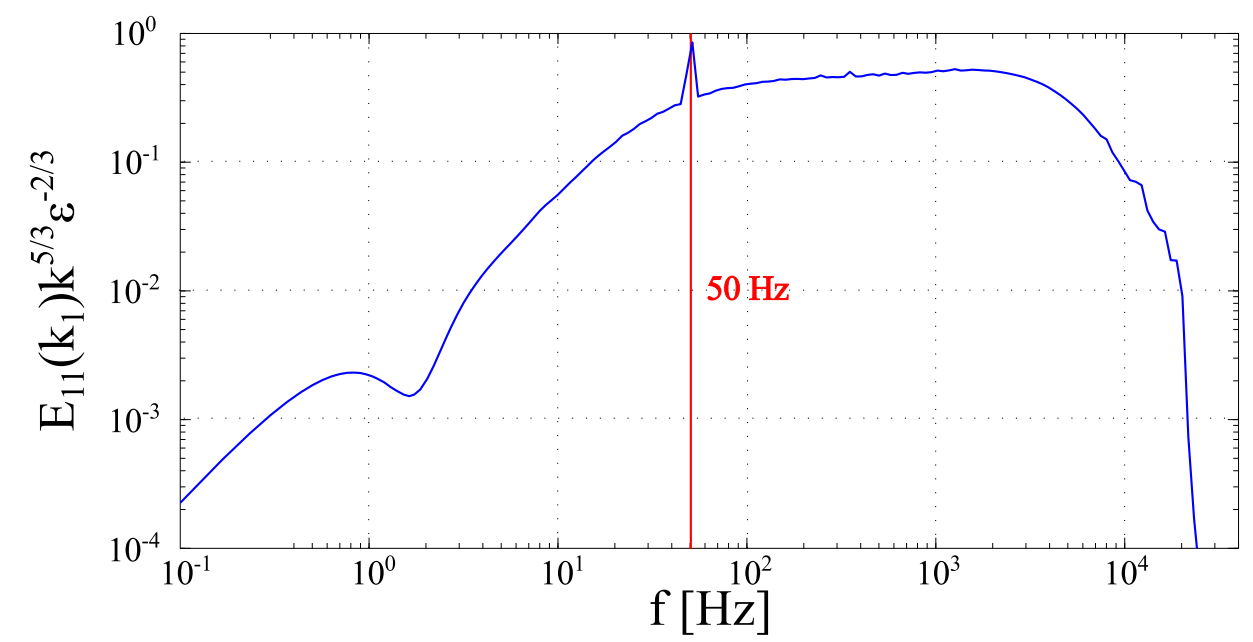

Figure 5.2: Compensated energy spectrum at $R_{\lambda}=320$ as a function of frequency. The data significantly affected by electric noise, stemming from power supplies, lights and computers located in the direct vicinity of the signal cables and measurement probes. Additionally, there is a large-scale defect in the energy spectrum.

Due to the gigantic size of the wind tunnel, the limited access to the test section and the amount of participating experiments in the ESWIRP project, an experimental setup was chosen in which the signal cables of the individual experiments were led to the measurement computers in close proximity to each other, to power cables and to computer power supplies. This resulted in a strong corruption of the measured signals at the utility frequency of $50 \mathrm{~Hz}$. This can be observed in the compensated energy spectrum in figure 5.2, in which a distinct sharp peak at $50 \mathrm{~Hz}$ emerges. The low-frequency defect in the spectrum might be related to pollution of the flow with small organic fibers settling on the measurement probes. Even though no contamination of the probes could be observed after the experiments, dampening of 
the signal by small objects of the corresponding size during the experiment cannot be ruled out as a potential cause of the energy defect.

Nevertheless, these large-scale problems in the measurement do not influence the small scales of interest here. With the given unfiltered access to the dissipation range, the energy dissipation rate can be estimated directly from the energy spectrum instead of relying on the structure functions. In an isotropic turbulent flow, the energy dissipation rate is given by (Batchelor 1953)

$$
\varepsilon=15 v \int_{0}^{\infty} k_{1}^{2} \cdot E_{11}\left(k_{1}\right) \mathrm{d} k_{1}
$$

Figure 5.3 shows the dissipation spectrum for a measurement in the dataset S1MA. As the dissipation range is well resolved, the spectrum drops to zero before noise starts to influence the shape of the small scale part in a significant way. Note that the plot is in semi-logarithmic coordinates, so that the peak at $50 \mathrm{~Hz}$ does not contribute significantly to the value of the integral in equation (5.1).

The dissipation rate obtained from the spectrum can then be used to test the estimation from the structure functions. Figure 5.4 shows the energy dissipation rate estimated by different means. While the estimation from Kolmogorov's equation agrees well with the direct computation from the dissipation spectrum, an estimation from the third-order structure function alone leads to a significant underestimation. Thus, to compute the energy dissipation rate in measurements that do not resolve the dissipation range, the corrected third-order structure function should be used. 


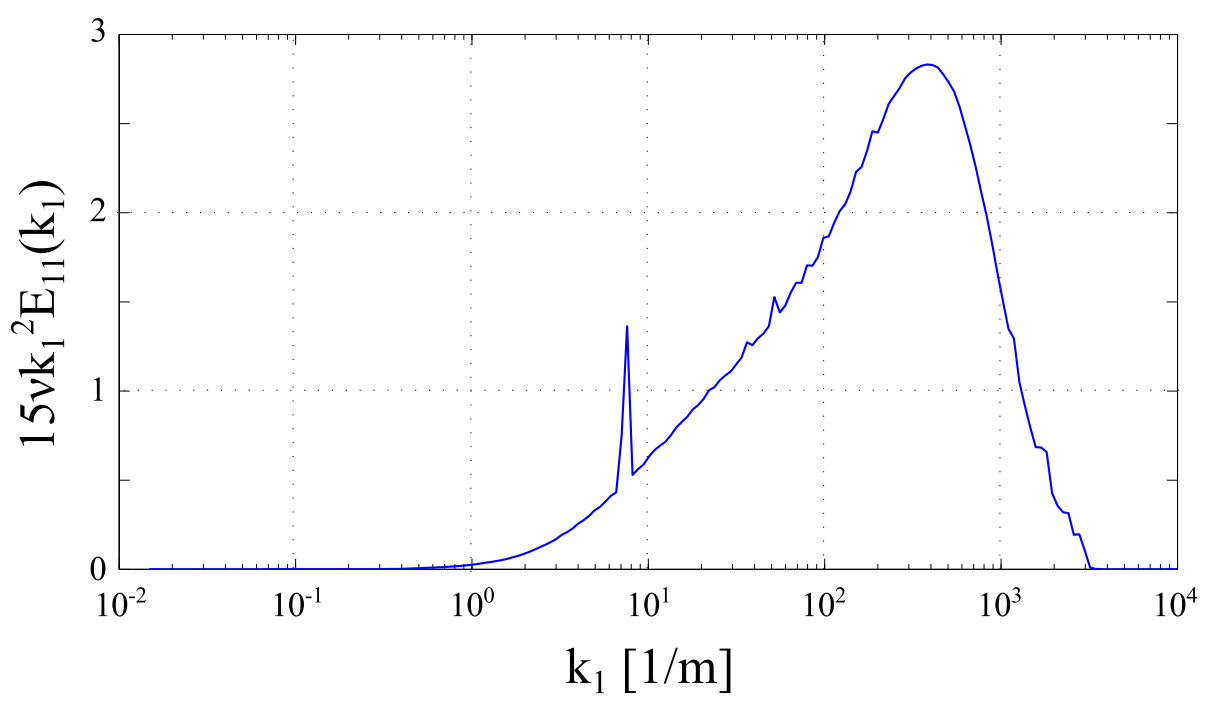

Figure 5.3: The one-dimensional energy dissipation spectrum. The integral over this spectrum is equivalent to the energy dissipation rate under the assumption of isotropy. The peak at $k_{1} \approx 7 \mathrm{~m}^{-1}$ stems from the aforementioned electric noise.

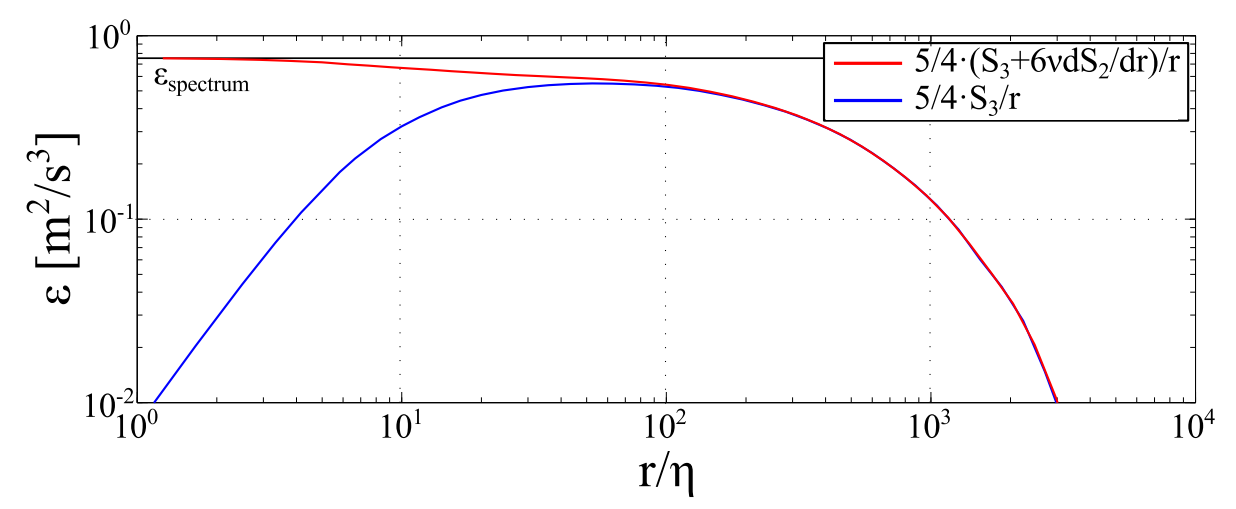

Figure 5.4: Comparison of the different methods of obtaining the dissipation rate. An estimation from the third-order structure function (blue) underestimates the value of $\varepsilon$. The results from the corrected third-order structure function (red) and from the dissipation spectrum (black) agree. 


\subsection{Statistics of the Velocity Derivatives}

Many features of turbulent flows, such as the decay of energy or the scaling properties of structure functions, can be investigated by means of velocities and velocity increments alone. However, one of the key quantities in turbulence, the instantaneous energy dissipation rate, is inherently a quantity of the velocity derivatives via $\varepsilon=\frac{v}{2} \sum_{i, j}\left(\frac{\partial u_{i}}{\partial x_{j}}+\frac{\partial u_{j}}{\partial x_{i}}\right)^{2}$. The distribution of the energy dissipation plays an important role in, e.g., modeling structure functions, as discussed in section 4.1. It is well known since Landau \& Lifschitz (1959) that the energy dissipation rate is a highly intermittent quantity. This can be seen directly in time series of turbulent velocity and of turbulent velocity derivatives. Figures 5.5 and 5.6 show times series of mean-substracted velocity and the approximated derivatives from the dataset S1MA, normalized by their standard deviations. The derivatives were computed by means of finite differences with a time difference $\Delta t=10^{-5} \mathrm{~s}$. In contrast to the turbulent velocity, the velocity derivative time series and the time series of its squares show strong intermittent bursts. Periods of relative quiescence alternate with sharp peaks. Additionally, the time series of the squares of the velocity derivatives is of interest. Using Taylor's hypothesis, temporal derivatives can be transformed into spatial derivatives. Thus, the square of the temporal velocity derivative can be seen as a surrogate for the energy dissipation rate $\varepsilon=15 v\left\langle(\partial u / \partial x)^{2}\right\rangle$. Hence, the rate at which energy is dissipated in turbulence is strongly intermittent as well. The strong intermittent bursts in measurements of the energy dissipation rate have been investigated carefully by Tsinober et al. (1992). 

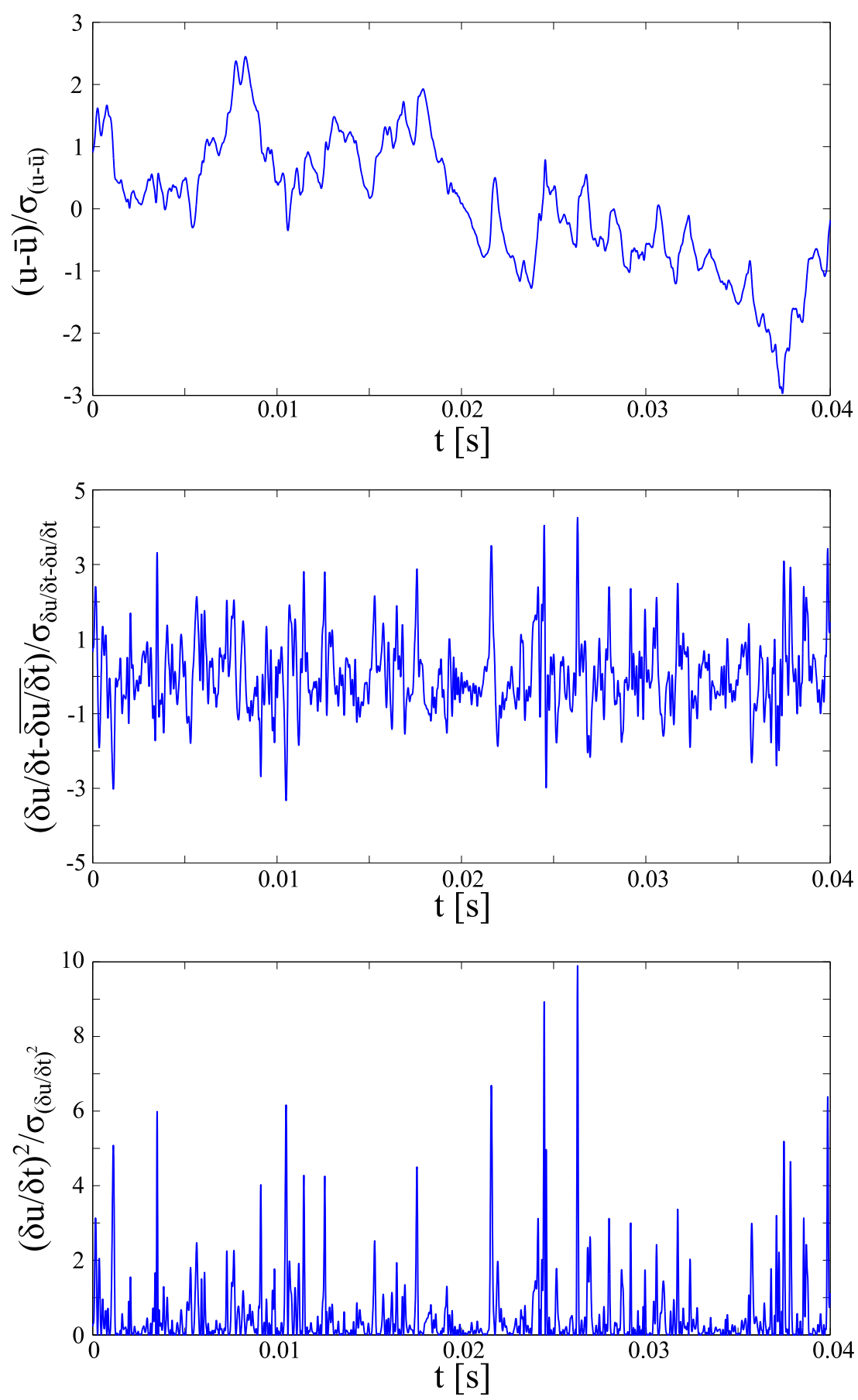

Figure 5.5: Short time series of velocity, velocity derivative and square of the velocity derivative at $R_{\lambda}=250$, normalized by their mean and standard deviations. The derivative time series show strong intermittent bursts comparable to those reported by Tsinober et al. (1992). 

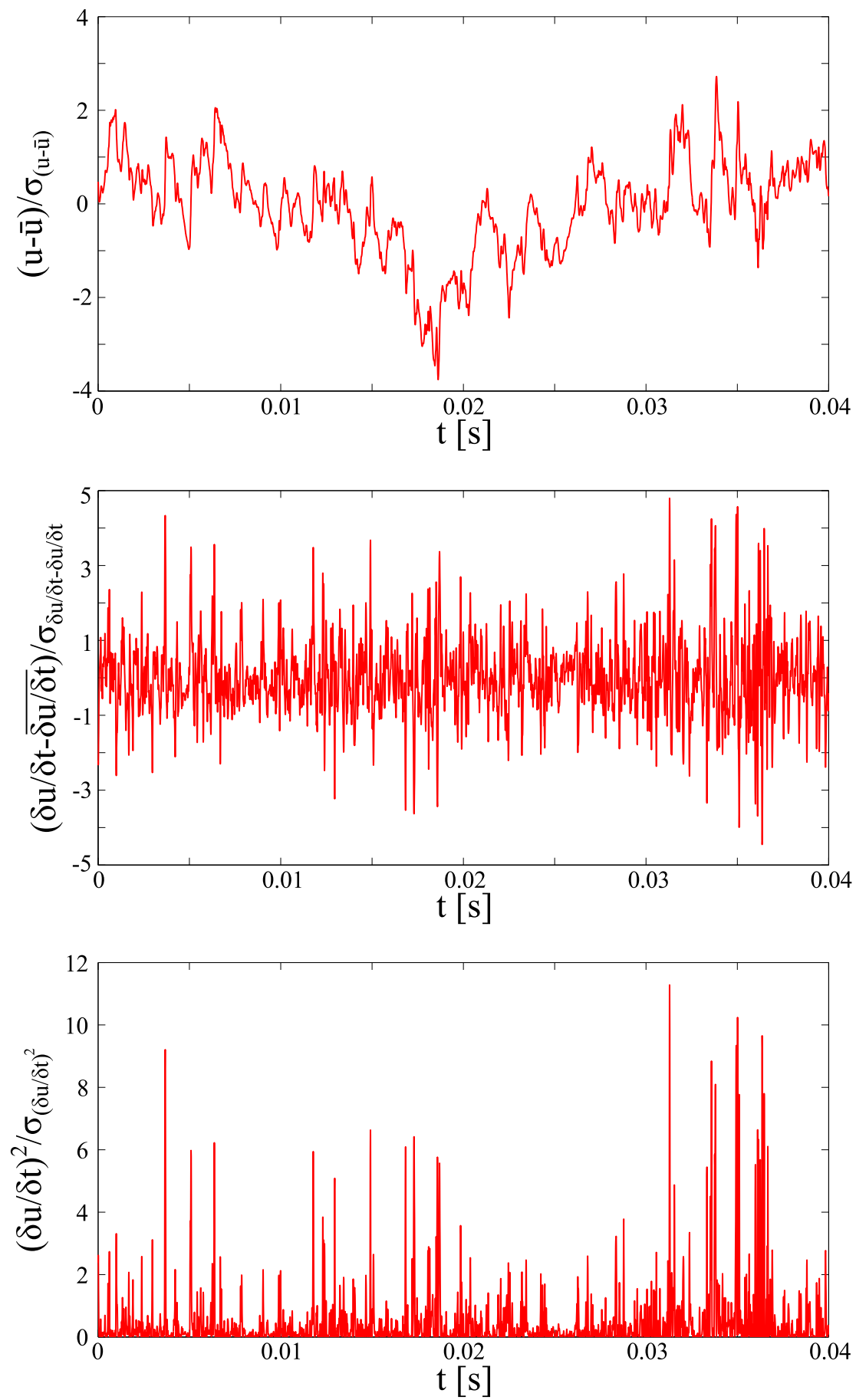

Figure 5.6: Short time series of velocity, velocity derivative and square of the velocity derivative at $R_{\lambda}=320$, normalized by their mean and standard deviations. The derivative time series show strong intermittent bursts comparable to those reported by Tsinober et al. (1992). 
In turbulent flows, single-point velocities are distributed close to a Gaussian distribution. Figure 5.7 shows the non-normalized distribution functions for the same datasets as in figures 5.5 and 5.6. The black dashed lines represent Gaussian fits to the colored data. The fit and the data agree. Deviations from a Gaussian behavior at large standard deviations from the mean might be attributed to the finite amount of the underlying statistics. Thus, no information about possible nonGaussianity can be obtained from this data. Extreme events in the velocity itself are unlikely to occur in turbulent flows. Following figures 5.5 and 5.6, to find a velocity four standard deviations higher or lower than the mean is almost four orders of magnitude more unlikely than to find a velocity of the mean speed. This Gaussianity is well-known and discussed in the standard textbooks (see, e.g., Davidson (2009)). Velocity increments, however, show significant deviations from Gaussian behavior for small separations (see, e.g., Castaing et al. (1990), Peinke et al. (1996), Friedrich \& Peinke (1997), Renner et al. (2001)). The shape of the probability density function of velocity increments, $\Delta u=u(t+\Delta t)-u(t)$, sensitively depends on the choice of the time difference, $\Delta t$. For $\Delta t \rightarrow 0$, the velocity differences, $\Delta u / \Delta t$, become identical to the velocity derivative. Figures 5.8 and 5.9 show the non-normalized distribution functions for the velocity differences and their squares for varying time differences $\Delta t$. For large $\Delta t$, the distribution functions are virtually Gaussian. However, for small time differences, one can observe a significant deviation from Gaussian behavior. The distributions have pronounced heavy tails indicating an increased likelihood for the occurrence of extreme events. The increasing skewness indicates a build-up of asymmetry of the increments with decreasing scale. 

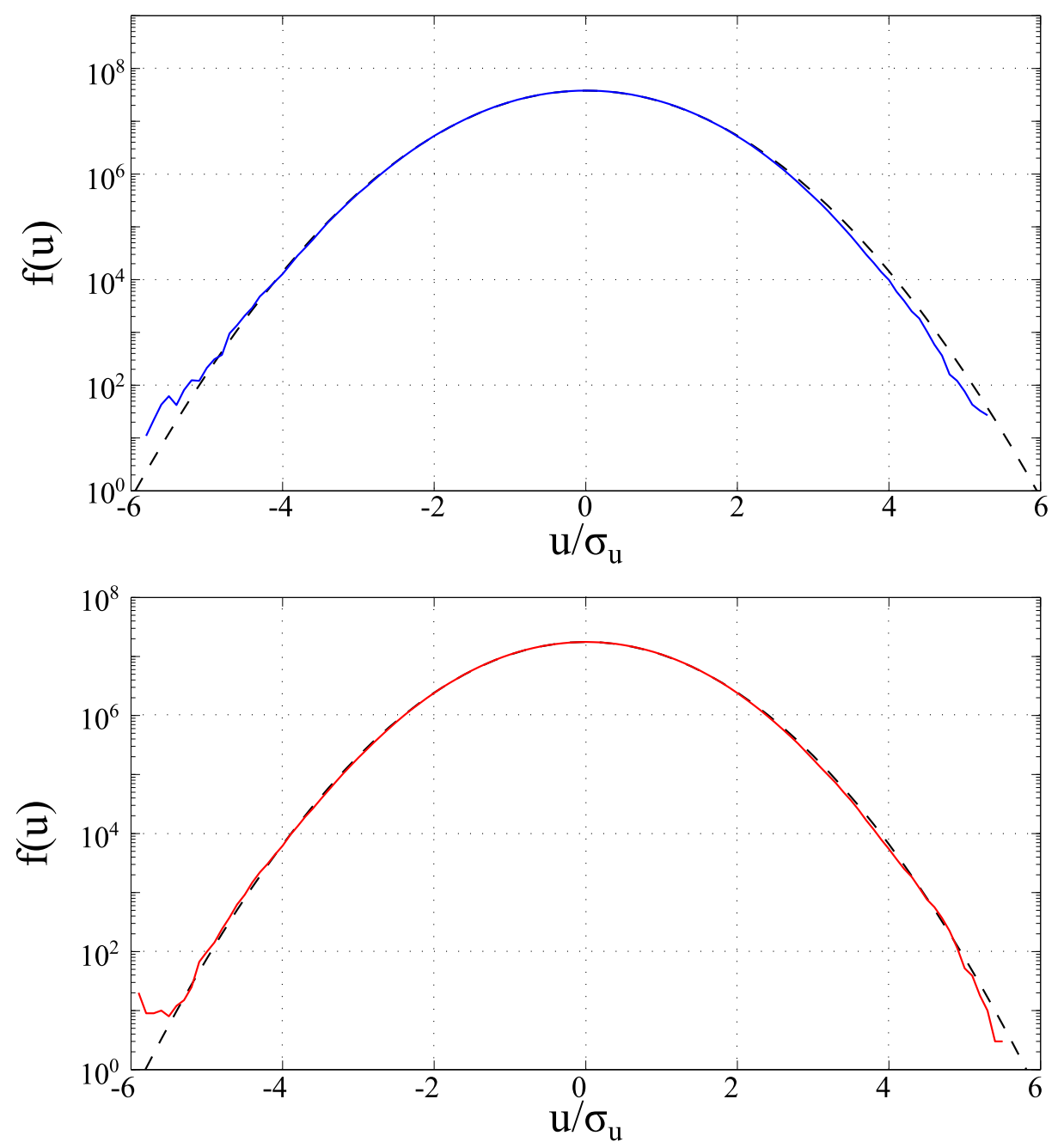

Figure 5.7: Non-normalized distribution function of velocity for a $R_{\lambda}=250$ (blue) and $R_{\lambda}=320$ (red) measurement from the dataset S1MA. The dashed black lines are Gaussian fits to the data. 

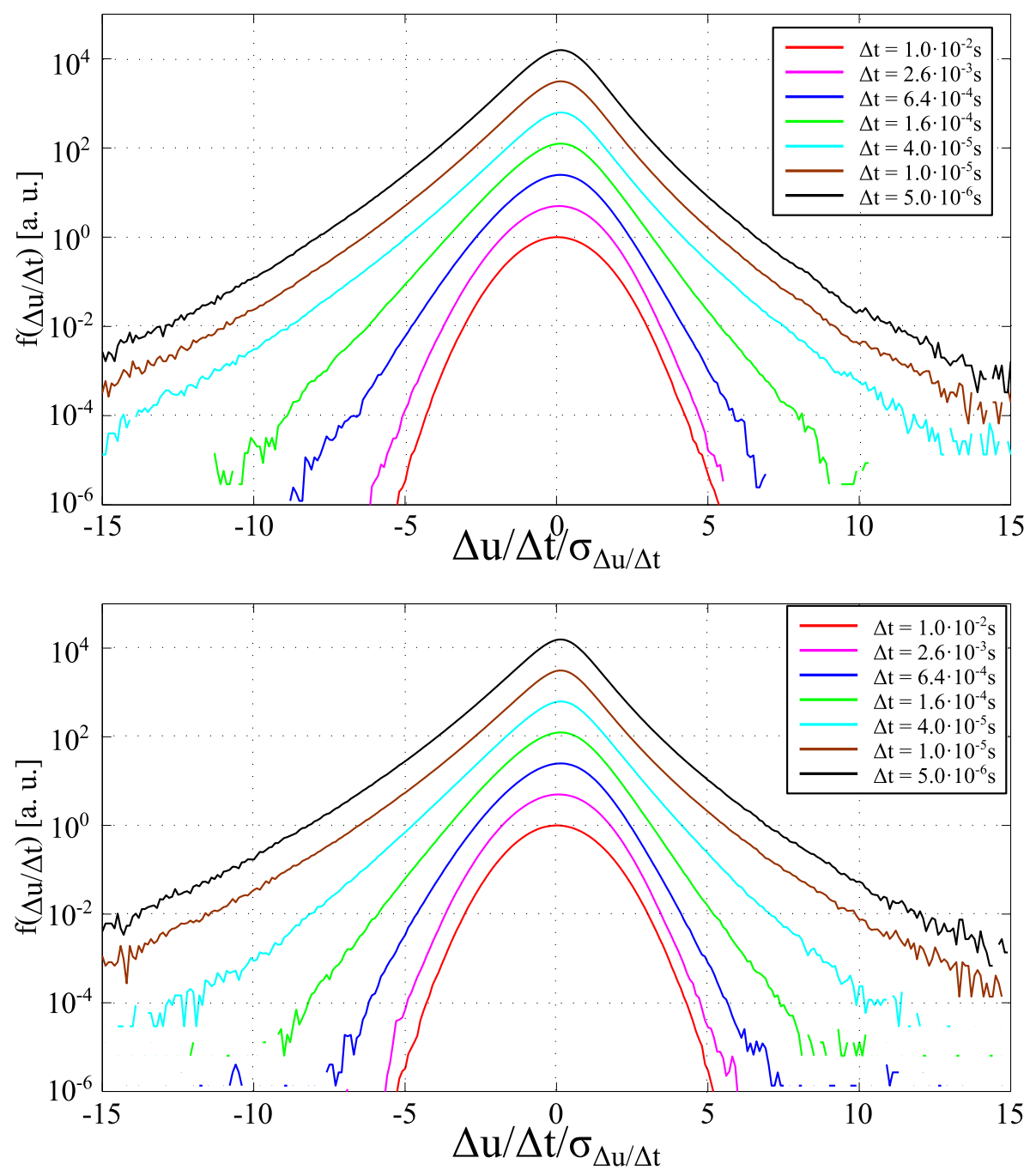

Figure 5.8: Non-normalized distribution function of velocity increments, for time increments between $\Delta t=5 \cdot 10^{-6} \mathrm{~s}$ and $\Delta t=1 \cdot 10^{-2} \mathrm{~s}$. For better visibility, the curves are shifted vertically with decreasing increments. The top figure corresponds to a measurement at $R_{\lambda}=250$, the bottom figure to a measurement at $R_{\lambda}=320$. 

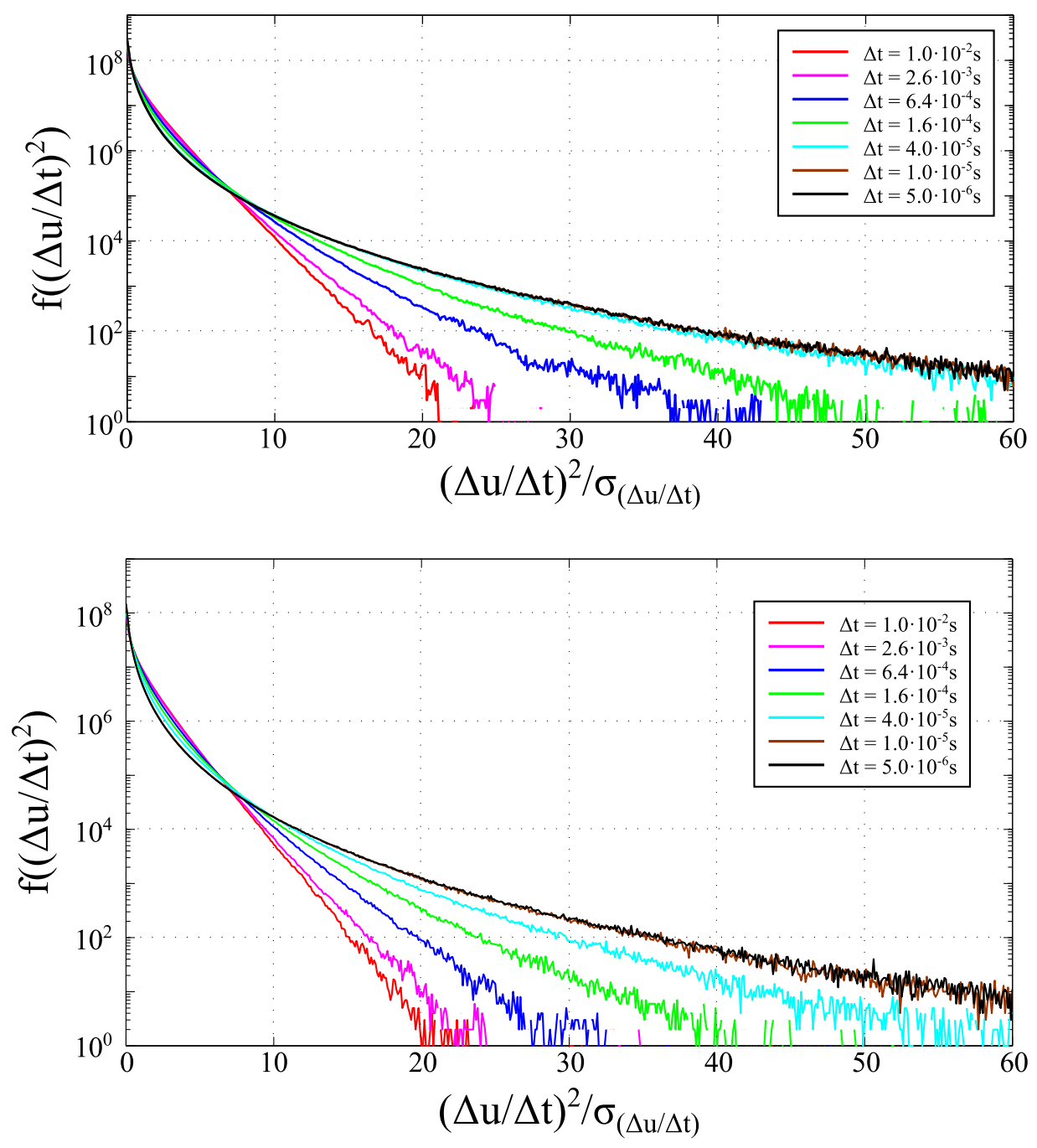

Figure 5.9: Non-normalized distribution function of the squared velocity increments used as a surrogate for the dissipation rate, for time increments between $\Delta t=5 \cdot 10^{-6} \mathrm{~s}$ and $\Delta t=1 \cdot 10^{-2} \mathrm{~s}$. The top figure corresponds to a measurement at $R_{\lambda}=250$, the bottom figure to a measurement at $R_{\lambda}=320$. 


\subsection{The Build-Up of non-Gaussianity}

To quantify the build-up of non-Gaussiantiy of the velocity differences with decreasing $\Delta t$ towards the velocity derivatives, it is useful to investigate ratios of structure functions. By the use of Taylor's hypothesis, temporal and spatial derivatives can be converted into each other. Thus, a description on how the skewness and flatness changes with scale is equivalent to a description on how they change with time. Key quantities to describe the non-Gaussianity of the distribution functions of the velocity derivatives are skewness, $S$, and flatness, $F$, given by

$$
\begin{aligned}
& S=\left\langle\left(\frac{\partial u}{\partial x}\right)^{3}\right\rangle /\left\langle\left(\frac{\partial u}{\partial x}\right)^{2}\right\rangle^{3 / 2} \\
& F=\left\langle\left(\frac{\partial u}{\partial x}\right)^{4}\right\rangle /\left\langle\left(\frac{\partial u}{\partial x}\right)^{2}\right\rangle^{2} .
\end{aligned}
$$

For a Gaussian distribution, the skewness and the flatness can be shown to be zero and three, respectively.

One can now write skewness of the velocity derivative distribution in terms of structure functions via

$$
\frac{S_{3}}{S_{2}^{3 / 2}}=\frac{r^{3} \cdot\left\langle(u(x+r)-u(x))^{3}\right\rangle}{r^{3} \cdot\left\langle(u(x+r)-u(x))^{2}\right\rangle^{3 / 2}} \stackrel{r \rightarrow 0}{=} S .
$$

Here, shifting the pre-multiplied factor of one, given in terms of $r^{3} / r^{3}$, is shifted 
into the respective averages and the limit of $r \rightarrow 0$ is taken.

The flatness can be analogously written as

$$
\frac{S_{4}}{S_{2}^{2}}=\frac{r^{4} \cdot\left\langle(u(x+r)-u(x))^{4}\right\rangle}{r^{4} \cdot\left\langle(u(x+r)-u(x))^{2}\right\rangle^{2}} \stackrel{r \rightarrow 0}{=} F .
$$

A variation in the ratio of the structure functions with respect to the scale thus provides quantitative information about the change of shape of the distribution functions with scale. Figure 5.10 shows the ratio $-S_{3} / S_{2}^{3 / 2}$ as a function of separation, normalized by the Kolmogorov length scale. For large separations, the skewness approaches its Gaussian value of zero.

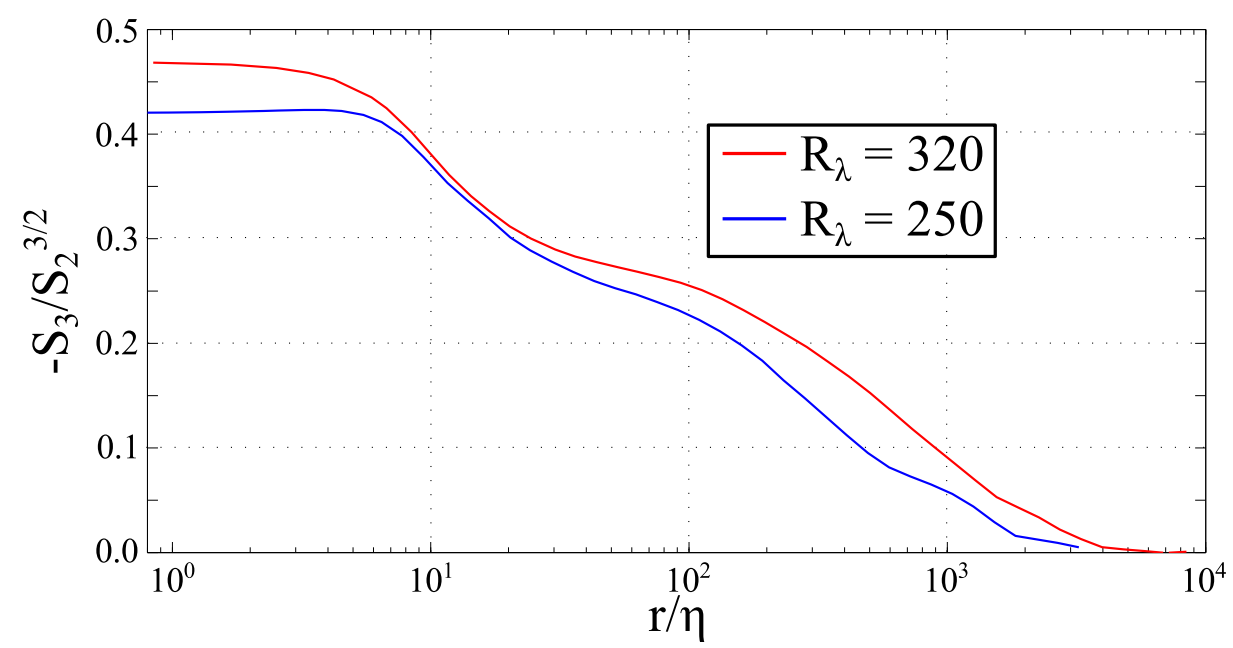

Figure 5.10: Dependence of the ratio of the third-order structure function to the second-order structure function to the power of $3 / 2$. For large separations, the ratio reaches the Gaussian value of 0 . For $r \rightarrow 0$, the ratio is equivalent to the skewness of the velocity derivatives. 
In the near-dissipation range, at $r / \eta \approx 20$, there is a significant increase of skewness. For $r / \eta<10$, the skewness approaches a constant value, the velocity derivative skewness. The resulting skewness is in agreement with the results from Van Atta \& Antonia (1980).

The scale-dependent ratio $S_{4} / S_{2}^{2}$ shown in figure 5.11 behaves in a similar manner. For large separations, it assumes the Gaussian value of three, while at $r / \eta \approx$ 20 , there is a rapid build-up of intermittency with scale. For the smallest scales, $r / \eta<10$, the scale-dependent flatness reaches a constant value, the flatness of the velocity derivatives. This build-up of intermittency is in agreement with a model by Chevillard et al. (2005) that links inertial range intermittency to the dissipation range by the use of an amplification law.

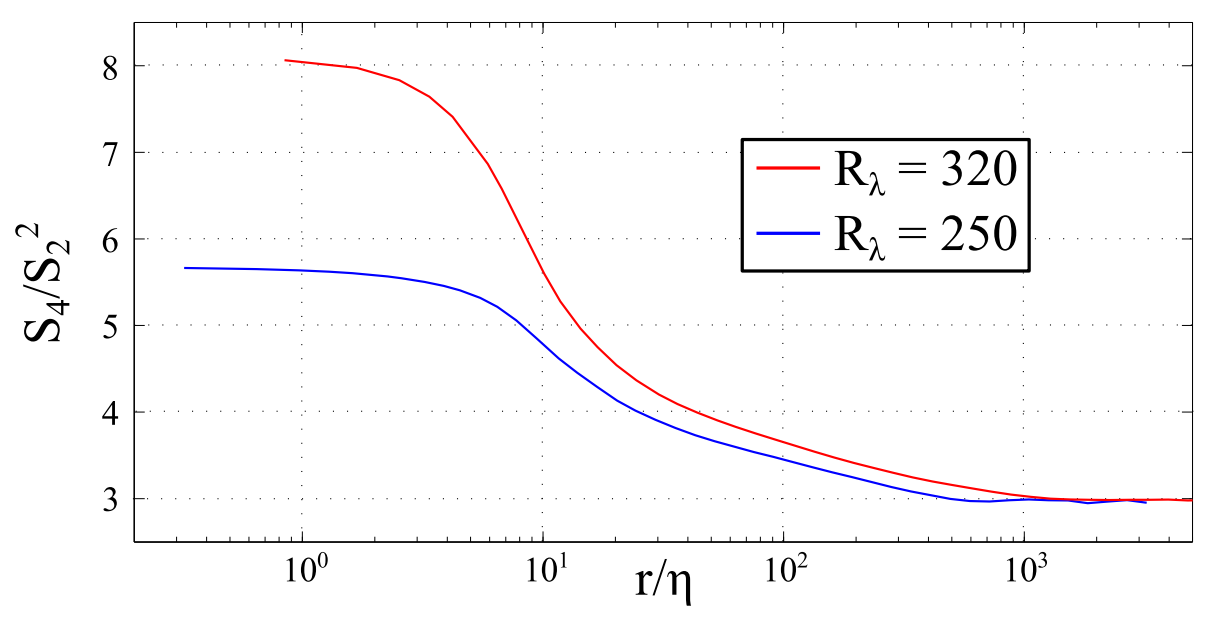

Figure 5.11: Dependence of the ratio of the fourth-order structure function to the square second-order structure function. For large separations, the ratio reaches the Gaussian value of three. For $r \rightarrow 0$, the ratio is equivalent to the velocity derivative flatness. 
These findings can be used to understand the change of shape of the velocity increment distribution functions in figure 5.8. The red and purple curves, corresponding to separations in the inertial range with $r / \eta>20$, resemble Gaussian distributions. With the green and cyan curves, the near-dissipation range is reached. The shape of the distributions rapidly develops heavy tails with strong deviations from Gaussian behavior. In the far-dissipation range (brown and black), the shape of the distributions does not change significantly with scale, agreeing with the findings in figure 5.11. 


\section{Discussion and Outlook}

Albeit of fundamental importance for the prediction of extreme events, aerodynamics and numerical modeling, the influence of the Reynolds number on many aspects of turbulent flows is not precisely known. In my thesis, I used the ability of the Variable Density Turbulence Tunnel at the Max-Planck-Institute for Dynamics and Self-Organization in Göttingen to change the Reynolds number over a wide range. Thus, I was able to experimentally investigate its role in key aspects of turbulence.

In chapter 3, I addressed the question whether the rate of decay of turbulent flows depends on the Reynolds number. There is a line of thought that at very high Reynolds numbers, a self-similar decay with constant Reynolds number emerges. With the results from chapter 3, this type of decay can be ruled out for realistically obtainable Reynolds numbers. Rather than the self-similar decay, the high-Reynolds number limit of decaying turbulence seems to be Saffmann's turbulence, originally connected to patches of turbulent with conserved linear momentum dominating the flow properties. This picture might have to be adjusted, as the large-scale structure of the energy spectrum in the experiments I conducted possibly disagrees with the predicted one. Two aspects of the results from chapter 3 are promising to be 
investigated in future research. While there is no approach to self-similar decay at high Reynolds numbers, there seems to be an approach towards Batchelor's turbulence at low Reynolds numbers. Even though the VDTT is a wind tunnel designed for high Reynolds number experiments, low Reynolds numbers can be realized using Helium as working gas in combination with a smaller grid with round grid bars. This lowers the minimal obtainable Reynolds number by at least one order of magnitude, making it possible to investigate a potential transition of Saffman's turbulence towards Batchelor's turbulence. The connection between the large-scale part of the three-dimensional energy spectrum and the rate of decay will have to be investigated in detail. This is to test the original theoretical predictions of conserved quantities being responsible for the rate of decay. A useful experimental method for this will be to use an active grid to be built in the VDTT that is capable of modifying the large-scale structure of the turbulent flow at will. With the long-time measurement abilities of the VDTT, a thorough investigation on this matter will be possible. The use of a to-be-developed three-wire nano-scale hot-wire might give direct access to the three-dimensional spectrum.

In chapter 4, I investigated the statistics of velocity increments in the inertial range and the predictions of scaling behavior in the structure functions. I found that neither scaling in the classical sense nor in the sense of Extended Self-Similarity is present in turbulent flows. There is a systematic deviation from scaling in turbulence. Dissipative effects extend much further into the inertial range than expected, completely universal with Reynolds number. The commonly used models for the shape of structure functions assume scaling properties. This line of thought 
has to be improved, models including the systematic deviations are needed. A more promising approach to describe the statistics of velocity increments might be by means of the velocity increment probability density functions instead of the structure functions. As these functions contain the complete statistical information about the velocity increments, using them to investigate the absence of scaling might be more fruitful.

In chapter 5, I used the data from the measurement campaign in Modane to investigate the build-up of intermittency towards the small scales. As spatial filtering did not play a role in the measurements, a large part of the dissipative range could be investigated. The general temporal limitations of CTA systems regarding electric noise at high frequencies, however, prevented an unfiltered access to the subKolmogorov scales. With improved, to-be-developed electronics, such as a constant current anemometer specifically built to work with extremely small nano-scale probes, the temporal resolution the measurements could be improved such that sub-Kolmogorov-scale statistics might be fully accessible in the VDTT at moderate Reynolds numbers. This would also give complete access to velocity derivative statistics. 


\section{Appendix}

\section{A Experimental Conditions}

\begin{tabular}{c|c} 
Dataset & Decay \\
\hline Probes & P11, Mini, NSTAP \\
Distance from the grid $[\mathrm{m}]$ & 1.5 to 8.3 \\
Sampling rate $[\mathrm{kHz}]$ & 60 \\
\# of positions & 50 \\
\# samples per position & $1.8 \cdot 10^{7}$ \\
Working gas & Air, $\mathrm{SF}_{6}$ \\
$p[\mathrm{bar}]$ & 0.5 to 15 \\
$v\left[\mathrm{~m}^{2} / \mathrm{s}^{2}\right]$ & $1.4 \cdot 10^{-7}$ to $3.3 \cdot 10^{-5}$ \\
\hline$U[\mathrm{~m} / \mathrm{s}]$ & 2.45 to 4.95 \\
$u^{\prime} / U$ & $1.58 \%$ to $3.56 \%$ \\
$\varepsilon\left[\mathrm{m}^{2} / \mathrm{s}^{3}\right]$ & $3.45 \cdot 10^{-3}$ to $1.77 \cdot 10^{-2}$ \\
$R \lambda$ & 21 to 1450 \\
$\eta[\mu \mathrm{m}]$ & 21 to 1600 \\
$\lambda[\mathrm{mm}]$ & 1.57 to 20.3 \\
$L[\mathrm{~mm}]$ & 115 to 254
\end{tabular}

Table 6.1: Conditions of the decay measurements for the dataset Decay. The magnitude of the derived quantities changes with distance from the grid. Given are the quantities at the largest distance from the grid, obtained with NSTAPs. 


\begin{tabular}{c|c|c} 
Dataset & Decay_Modified & Decay_Near \\
\hline Probes & P11, Mini, NSTAP & P11 \\
Grid distance [m] & 1.5 to 8.3 & 0.034 to 1.86 \\
Sampling rate $[\mathrm{kHz}]$ & 60 & 60 \\
\# positions & 50 & 14 \\
\# samples per position & $1.8 \cdot 10^{7}$ & $1.8 \cdot 10^{7}$ \\
Working gas & Air, $\mathrm{SF}_{6}$ & Air \\
$p[\mathrm{bar}]$ & 1 to 15 & 1 \\
$v\left[\mathrm{~m}^{2} / \mathrm{s}^{2}\right]$ & $1.4 \cdot 10^{7}$ to $1.55 \cdot 10^{-5}$ & $1.55 \cdot 10^{-5}$ \\
\hline$U[\mathrm{~m} / \mathrm{s}]$ & 4.2 to 4.3 & 3.9 \\
$u^{\prime} / U$ & $1.0 \%$ to $3.4 \%$ & $4 \%$ \\
$\varepsilon\left[\mathrm{m}^{2} / \mathrm{s}^{3}\right]$ & 0.002 to 0.02 & 0.1 \\
$R \lambda$ & 70 to 1500 & 80 \\
$\eta[\mu \mathrm{m}]$ & 20 to 1250 & 430 \\
$\lambda[\mathrm{mm}]$ & 1.5 to 20 & 7.4 \\
$L[\mathrm{~mm}]$ & 124 to 138 & 70
\end{tabular}

Table 6.2: Conditions of the decay measurements for the datasets Decay_Modified and Decay_Near. Given are the quantities at the largest distance from the grid.

\begin{tabular}{c|c} 
Dataset & Statistic_Medium \\
\hline Probes & NSTAP \\
Grid distance $[\mathrm{m}]$ & 7.1 \\
Sampling rate $[\mathrm{kHz}]$ & 60 and 200 \\
\# samples & $1.8 \cdot 10^{7}$ to $2.0 \cdot 10^{8}$ \\
Working gas & Air, $\mathrm{SF}_{6}$ \\
$p[\mathrm{bar}]$ & 1 to 15 \\
$v\left[\mathrm{~m}^{2} / \mathrm{s}^{2}\right]$ & $1.4 \cdot 10^{-7}$ to $1.5 \cdot 10^{-5}$ \\
\hline$U[\mathrm{~m} / \mathrm{s}]$ & 1.32 to 4.31 \\
$u^{\prime} / U$ & $1.99 \%$ to $4.38 \%$ \\
$\varepsilon\left[\mathrm{m}^{2} / \mathrm{s}^{3}\right]$ & 0.0003 to 0.035 \\
$R_{\lambda}$ & 50 to 1600 \\
$\eta[\mu \mathrm{m}]$ & 17 to 1080 \\
$\lambda[\mathrm{mm}]$ & 1.44 to 18.7 \\
$L[\mathrm{~mm}]$ & 72.7 to 150
\end{tabular}

Table 6.3: Experimental conditions of the dataset Statistic_Medium 


\begin{tabular}{c|c|c} 
Dataset & Statistic_Large & Statistic_Huge \\
\hline Probes & P11, Mini, NSTAP & P11, Mini, NSTAP \\
Grid distance [m] & 8.3 & 6.9 and 8.3 \\
Sampling rate $[\mathrm{kHz}]$ & 60 & 60 \\
\# samples & $1.3 \cdot 10^{9}$ to $1.9 \cdot 10^{9}$ & $1.0 \cdot 10^{10}$ to $1.5 \cdot 10^{10}$ \\
Working gas & Air, $\mathrm{SF}_{6}$ & $\mathrm{SF}_{6}$ \\
$p[\mathrm{bar}]$ & 1 to 14.5 & 1 to 15 \\
$v\left[\mathrm{~m}^{2} / \mathrm{s}^{2}\right]$ & $1.5 \cdot 10^{-7}$ to $1.55 \cdot 10^{-5}$ & $1.4 \cdot 10^{-7}$ to $2.6 \cdot 10^{-6}$ \\
\hline$U[\mathrm{~m} / \mathrm{s}]$ & 3.46 to 5.11 & 4.01 to 4.34 \\
$u^{\prime} / U$ & $1.57 \%$ to $3.45 \%$ & $2.7 \%$ to $3.6 \%$ \\
$\varepsilon\left[\mathrm{m}^{2} / \mathrm{s}^{3}\right]$ & 0.003 to 0.015 & 0.012 to 0.024 \\
$R_{\lambda}$ & 110 to 1450 & 300 to 1600 \\
$\eta[\mu \mathrm{m}]$ & 22 to 1030 & 19 to 191 \\
$\lambda[\mathrm{mm}]$ & 1.61 to 21.1 & 1.45 to 6.73 \\
$L[\mathrm{~mm}]$ & 103 to 138 & 126 to 129
\end{tabular}

Table 6.4: Experimental conditions of the datasets Statistic_Large and Statistic_Huge.

\begin{tabular}{c|c} 
Dataset & S1MA \\
\hline Probes & NSTAP \\
Sampling rate $[\mathrm{kHz}]$ & 200 \\
\# samples & $4.4 \cdot 10^{8}$ to $9.6 \cdot 10^{8}$ \\
Working gas & Air \\
$p[\mathrm{bar}]$ & 1 \\
$v\left[\mathrm{~m}^{2} / \mathrm{s}^{2}\right]$ & $1.5 \cdot 10^{-5}$ \\
\hline$U[\mathrm{~m} / \mathrm{s}]$ & 21 to 43 \\
$u^{\prime} / U$ & $1.2 \%$ to $1.7 \%$ \\
$\varepsilon\left[\mathrm{m}^{2} / \mathrm{s}^{3}\right]$ & 0.26 to 0.75 \\
$R_{\lambda}$ & 250 to 320 \\
$\eta[\mu \mathrm{m}]$ & 260 to 330 \\
$\lambda[\mathrm{mm}]$ & 9 to 10 \\
$L_{e}[\mathrm{~mm}]$ & 129 to 148
\end{tabular}

Table 6.5: Experimental conditions of the measurements conducted in the S1MA. 


\section{B Scaling Exponents Comparison}

\begin{tabular}{c|c|c|c|c|c} 
Reference & $\zeta_{4,2}$ & $\zeta_{6,2}$ & $\zeta_{8,2}$ & $\zeta_{10,2}$ & $\zeta_{12,2}$ \\
\hline VDTT data & 0.5934 & 1.092 & 1.499 & 1.82 & 2.05 \\
Statistical uncertainty & \pm 0.0003 & \pm 0.001 & \pm 0.002 & \pm 0.01 & \pm 0.02 \\
$\Delta$ & \pm 0.0074 & \pm 0.027 & \pm 0.063 & \pm 0.1 & \pm 0.14 \\
\hline Cao et al. (1996) & 0.584 & 1.077 & 1.493 & & \\
Toschi et al. (1999) & 0.58 & 1.08 & & & \\
Boratav \& Pelz (1997) & 0.5867 & 1.0778 & 1.4903 & 1.8463 & 2.1673 \\
& 0.5887 & 1.0873 & 1.5192 & 1.9069 & 2.2683 \\
Vincent \& Meneguzzi (1991) & 0.5846 & 1.0749 & 1.4896 & 1.8513 & 2.1817 \\
Gotoh (2013) & 0.5888 & 1.0873 & 1.5182 & 1.9033 & 2.2598 \\
& 0.5845 & 1.0737 & 1.4850 & 1.8394 & 2.1568 \\
& 0.604 & 1.13 & 1.52 & 1.90 & 2.23
\end{tabular}

Table 6.6: Relative scaling exponents measured in the VDTT compared to DNS. 


\begin{tabular}{c|c|c|c|c|c} 
Reference & $\zeta_{4,2}$ & $\zeta_{6,2}$ & $\zeta_{8,2}$ & $\zeta_{10,2}$ & $\zeta_{12,2}$ \\
\hline VDTT data & 0.5934 & 1.092 & 1.499 & 1.82 & 2.05 \\
Statistical uncertainty & \pm 0.0003 & \pm 0.001 & \pm 0.002 & \pm 0.01 & \pm 0.02 \\
$\Delta$ & \pm 0.0074 & \pm 0.027 & \pm 0.063 & \pm 0.1 & \pm 0.14 \\
\hline Kolmogorov (1941a) & 0.6667 & 1.333 & 2.000 & 2.67 & 3.00 \\
Kolmogorov (1962) & 0.5963 & 1.099 & 1.508 & 1.82 & 2.04 \\
Andrews et al. $(1989)$ & 0.6247 & 1.086 & 1.473 & 1.80 & 2.08 \\
Frisch et al. (1978) & 0.4510 & 0.902 & 1.353 & 1.80 & 2.25 \\
Benzi et al. (1984) & 0.9154 & 1.266 & 1.541 & 1.74 & 1.89 \\
Kida (1991) & 0.5928 & 1.092 & 1.500 & 1.82 & 2.05 \\
Meneveau \& Sreenivasan (1987a) & 0.5702 & 1.041 & 1.445 & 1.81 & 2.15 \\
She \& Lévêque (1994) & 0.5838 & 1.082 & 1.515 & 1.90 & 2.24 \\
Dubrulle (1994) & 0.5936 & 1.093 & 1.500 & 1.82 & 2.05
\end{tabular}

Table 6.7: Relative scaling exponents measured in the VDTT compared to theoretical models. The parameters in the models were used to fit the models to the VDTT data. 


\begin{tabular}{c|c|c|c|c|c} 
Reference & $\zeta_{4,2}$ & $\zeta_{6,2}$ & $\zeta_{8,2}$ & $\zeta_{10,2}$ & $\zeta_{12,2}$ \\
\hline VDTT data & 0.5934 & 1.092 & 1.499 & 1.82 & 2.05 \\
Statistical uncertainty & \pm 0.0003 & \pm 0.001 & \pm 0.002 & \pm 0.01 & \pm 0.02 \\
$\Delta$ & \pm 0.0074 & \pm 0.027 & \pm 0.063 & \pm 0.1 & \pm 0.14 \\
\hline Benzi et al. (1993) & 0.56 & 0.95 & 1.44 & & \\
Benzi et al. (1995) & 0.58 & 1.08 & 1.53 & & \\
Sreenivasan \& Dhruva (1998) & 0.55 & 1.00 & 1.34 & 1.67 & \\
Stolovitzky \& Sreenivasan (1993) & 0.55 & 1.02 & 1.42 & 1.82 & 2.20 \\
Shen \& Warhaft (2002) & 0.58 & 1.09 & 1.53 & 1.95 & 2.36 \\
Antonia \& Pearson (1997) & 0.59 & 1.08 & 1.52 & & \\
Belin et al. (1996) & 0.59 & 1.08 & 1.49 & & \\
Anselmet et al. (1984) & 0.60 & 1.10 & 1.53 & & \\
& 0.59 & 1.03 & 1.31 & & \\
Maurer et al. (1994) & 0.56 & 1.01 & 1.38 & 1.60 & 1.8 \\
& 0.62 & 1.09 & 1.56 & 1.93 & 2.23 \\
van de Water \& Herweijer (1999) & 0.62 & 1.09 & 1.56 & 1.89 & 2.03 \\
& 0.62 & 1.09 & 1.51 & 1.88 & 2.13 \\
& 0.6 & 1.1 & 1.3 & & \\
& 0.57 & 1.01 & 1.31 & 1.50 & 1.62 \\
& & 1.02 & 1.36 & 1.62 & 1.82
\end{tabular}

Table 6.8: Relative scaling exponents measured in the VDTT compared to experimental data. 


\section{Bibliography}

ACHENBACH, E. 1972 Experiments on the flow past spheres at very high reynolds numbers. J. Fluid Mech. 54, 565-575.

Andrews, L. C., Phillips, R. L., Shivamoggi, B. K., Beck, J. K. \& Joshi, M. L. 1989 A statistical theory for the distribution of energy dissipation in intermittent turbulence. Phys. Fluids A 1, 999-1006.

Anselmet, F., Gagne, Y. \& Hopfinger, E. J. 1984 High-order velocity structure functions in turbulent shear flows. J. Fluid Mech. 140, 63-69.

Antonia, R. A. \& Burattini, P. 2006 Approach to the 4/5 law in homogeneous isotropic turbulence. J. Fluid Mech. 550, 175-184.

Antonia, R. A. \& PeArson, B. R. 1997 Scaling exponents for turbulent velocity and temperature increments. Europhys. Lett. 40, 123-128.

Antonia, R. A., Pearson, B. R. \& Zhou, T. 2000 Reynolds number dependence of second-order velocity structure functions. Phys. Fluids 12, 3000-3006. 
Antonia, R. A., Smalley, R. J., Zhou, T., Anselmet, F. \& Danaila, L. 2003 Similarity of energy structure functions in decaying homogeneous isotropic turbulence. J. Fluid Mech. 487, 245-269.

Argyris, J., FAust, G., HaAse, M. \& Friedrich, R. 2010 Die Erforschung des Chaos. Springer-Verlag.

Arwatz, G., FAn, Y., BAhri, C. \& Hultmark, M. 2015 Development and characterization of a nano-scale temperature sensor (t-nstap) for turbulent temperature measurements. Meas. Sci. Technol. 26, 035103.

Ashok, A., Bailey, S. C. C., Hultmark, M. \& Smits, A. J. 2012 Hot-wire spatial resolution effects in measurements of grid-generated turbulence. Exp. Fluids 53, 1713-1722.

Bailey, S. C. C., Kunkel, G. J., Hultmark, M., Vallikivi, M., Hill, J. P., Meyer, K. A., TsAy, C., Arnold, C. B. \& Smits, A. J. 2010 Turbulence measurements using a nanoscale thermal anemometry probe. J. Fluid Mech. $\mathbf{6 6 3}$, $160-179$.

BATCHELOR, G. K. 1950 On the spontaneous magnetic field in a conducting liquid in turbulent motion. Proc. Roy. Soc. Lond. A 201, 405-416.

BAtChelor, G. K. 1953 The Theory of Homogeneous Turbulence. Cambridge University Press.

Batchelor, G. K. \& Townsend, A. A. 1947 Decay of vorticity in isotropic turbulence. Proc. Roy. Soc. Lond. A 190, 534-550. 
Batchelor, G. K. \& Townsend, A. A. $1948 a$ Decay of isotropic turbulence in the initial period. Proc. Roy. Soc. Lond. A 193, 539-558.

Batchelor, G. K. \& Townsend, A. A. $1948 b$ Decay of turbulence in the final period. Proc. Roy. Soc. Lond. A 194, 527-543.

Belin, F., Tabeling, P. \& Willaime, H. 1996 Exponents of the structure functions in a low temperature helium experiment. Physica D 93, 52-63.

Benzi, R., Ciliberto, S., Baudet, C. \& Chavarria, G. R. 1995 On the scaling of three-dimensional homogeneous and isotropic turbulence. Physica $D$ 80, 385-398.

Benzi, R., Ciliberto, S., Tripiccione, R., Baudet, C., Massaioli, F. \& SuCCI, S. 1993 Extended self-similarity in turbulent flows. Phys. Rev. E 48, R29-R32.

Benzi, R., Paladin, G., PArisi, G. \& Vulpiani, A. 1984 On the multifractal nature of fully developed turbulence and chaotic systems. J. Phys. A: Math. Gen. 17, 3521-3531.

Bewley, G. P., Lathrop, D. P., MaAs, L. R. M. \& Sreenivasan, K. R. 2007 Inertial waves in rotating grid turbulence. Phys. Fluids 19, 071701.

Bodenschatz, E., Bewley, G. P., Nobach, H., Sinhuber, M. \& Xu, H. 2014 Variable density turbulence tunnel facility. Rev. Sci. Instrum. 85, 093908. 
Bodenschatz, E., Malinowski, S. P., Shaw, R. A. \& Stratmann, F. 2010 Can we understand clouds without turbulence? Science 327, 970-971.

Boratav, O. N. \& Pelz, R. B. 1997 Structures and structure functions in the inertial range of turbulence. Phys. Fluids 9, 1400-1415.

Burattini, P., Lavoie, P., Agrawal, A., Djenidi, L. \& Antonia, R. A. 2006 Power law of decaying homogeneous isotropic turbulence at low reynolds number. Phys. Rev. E 73, 066304.

CaO, N., Chen, S. \& She, Z.-S. 1996 Scalings and relative scalings in the navier-stokes turbulence. Phys. Rev. Lett. 76, 3711-3714.

Castaing, B., Gagne, Y. \& Hopfinger, E. J. 1990 Velocity probability density functions of high reynolds number turbulence. Physica D 46, 177-200.

Chevillard, L., Castaing, B. \& LÉvÊQue, E. 2005 On the rapid increase of intermittency in the near-dissipation range of fully developed turbulence. Eur. Phys. J. B 45, 561-567.

Choudhari, M. M., Lockrad, D. P. Macaraeg, M. G., Singer, B. A. \& StREetT, C. L. 2002 Aeroacoustic experiments in the langley low-turbulence pressure tunnel. Tech. Rep.. NASA.

Comte-Bellot, G. 1976 Hot-wire anemometry. Annu. Rev. Fluid Mech. 8, 209_ 231. 
Comte-Bellot, G. \& Corrsin, S. 1966 The use of a contraction to improve the isotropy of grid-generated turbulence. J. Fluid Mech. 25, 657-682.

Corrsin, S. 1942 Decay of turbulence behind three similar grids. PhD thesis, California Institute of Technology.

DAVIDSON, P. A. 2004 turbulence. Oxford University Press.

DAVIDSON, P. A. 2009 The role of angular momentum conservation in homogeneous turbulence. J. Fluid Mech. 632, 329-358.

DAVIDSON, P. A. 2011 The minimum energy decay rate in quasi-isotropic grid turbulence. Phys. Fluids 23, 085108.

von Doenhoff, A. E. \& Aвbоtт, F. T. J. 1947 The langley two-dimensional low-turbulence pressure tunnel. Tech. Rep. 1283. Langley Memorial Aeronautical Laboratory.

Van Doorn, E., White, C. M. \& Sreenivasan, K. R. 1999 The decay of grid turbulence in polymer and surfactant solutions. Phys. Fluids 11, 2387-2393.

DRYDEN, H. L. 1973 A review of the statistical theory of turbulence. Quart. appl. Math. 1, 7-42.

Dryden, H. L., Schubauer, G. B., Mock, W. C. \& Skramstad, H. K. 1937 Measurements of the intensity and scale of wind tunnel turbulence and their relation to the critical reynolds number of spheres. Tech. Rep. 581. NACA. 
DUBRULle, B. 1994 Intermittency in fully developed turbulence: Log-poisson statistics and generalized scale covariance. Phys. Rev. Lett. 73, 959-962.

EFron, B. 1979 Bootstrap methods: Another look at the jacknife. The Annals of Statistics 7, 1-26.

Eyink, G. L. \& ThOMSON, D. J. 2000 Free decay of turbulence and breakdown of self-similarity. Phys. Fluids 12, 477-479.

FRIEDRICH, R. \& PEINKE, J. 1997 Description of a turbulent cascade by a fokkerplanck equation. Phys. Rev. Lett. 78, 863-866.

FRISCH, U. 1995 Turbulence. Cambridge University Press.

Frisch, U., Sulem, P.-L. \& NelKin, M. 1978 A simple dynamical model of intermittent fully developed turbulence. J. Fluid Mech. 87, 719-736.

Frisch, U. \& Vergassola, M. 1993 New Approaches and Concepts in Turbulence. Monte Veritá.

Försching, H., Melzer, E. \& Schewe, G. 1981 Ein neuer windkanal für gebäudeaerodynamische und-aeroelastische untersuchungen bei reynoldszahlen bis $10^{7}$. Tech. Rep.. DFVLR-AVA.

GeOrge, W. K. 1992 The decay of homogeneous isotropic turbulence. Phys. Fluids A 4, 1492-1509.

Gotoн, T. 2013 Private communication . 
GÖRTLER, H. 1940 Über den einfluß der wandkrümmung auf die entstehung der turbulenz. Z. angew. Math. Mech. 20, 138-147.

Hawthorne, W. R. 1951 Secondary circulation in fluid flows. Proc. Roy. Soc. Lond. A 206, 374-387.

He, X., Funfschilling, D., Nobach, H., Bodenschatz, E. \& Ahlers, G. 2012 Transition to the ultimate state of turbulent rayleigh-bénard convection. Phys. Rev. Lett. 108, 024502.

HERWIJER, J. \& VAN DE WATER, W. 1995 Universal shape of scaling functions in turbulence. Phys. Rev. Lett. 74, 4651-4654.

Hoogland, J. H. B., Van Den Berg, H. R. \& Trappeniers, N. J. 1985 Measurements of the viscosity of sulfur hexaflouride up to 100 bar by a capillaryflow viscometer. Physica A 134, 169-192.

Huisman, S. G., van Gils, D. P. M., Grossmann, S., Sun, C. \& Lohse, D. 2012 Ultimate turbulent taylor-couette flow. Phys. Rev. Lett. 108, 024501.

Hultmark, M., Vallikivi, M., Bailey, S. C. C. \& Smits, A. J. 2012 Turbulent pipe flow at extreme reynolds numbers. Phys. Rev. Lett. 108, 094501.

Hurst, D. \& VAssilicos, J. C. 2007 Scalings and decay of fractal-generated turbulence. Phys. Fluids 19, 035103. 
Hutchins, N., Monty, J. P., Baidya, R., Hultmark, M. \& Smits, A. J. 2012 A direct measure of the frequency response of hot-wire anemometers. In 18th Australasian Fluid Mechanics Conference. Launceston, Australia.

IshidA, T., DAVIDSON, P. A. \& KANEDA, Y. 2006 On the decay of isotropic turbulence. J. Fluid Mech. 564, 455-475.

JACOBS, E. N. \& Аввот, I. H. 1933 The n. a. c. a. variable-density wind tunnel. Tech. Rep. 416. National Advisory Committee for Aeronautics.

JØRGENSEN, F. E. 2001 How to measure turbulence with hot-wire anemometers a practical guide. Dantec Dynamics.

DE KÁRMÁn, T. \& HowARTh, L. 1938 On the statistical theory of isotropic turbulence. Proc. Roy. Soc. Lond. A 164, 192-215.

KhOlmyansky, M. \& TsinOBER, A. 2009 On an alternative explanation of anomalous scaling and how well-defined is the concept of inertial range. Phys. Lett. A 373, 2364-2367.

KIDA, S. 1991 Log-stable distribution and intermittency of turbulence. J. Phys. Soc. JPN. 60, 5-8.

KING, L. V. 1914 On the convection of heat from small cylinders in a stream of fluid: Determination of the convection constants of small platinum wires with applications to hot-wire anemometry. Phil. Trans. Roy. Soc. Lond. A 214, $373-432$. 
Kistler, A. L. \& VRebalovich, T. 1966 Grid turbulence at large reynolds numbers. J. Fluid Mech. 26, 37-47.

Kolmogorov, A. N. 1941a Dissipation of energy in locally isotropic turbulence. Dokl. Akad. Nauk SSSR 32, 16-18.

Kolmogorov, A. N. $1941 b$ The local structure of turbulence in incompressible viscous fluid for very large reynolds numbers. Dokl. Akad. Nauk SSSR 30, 299_ 303.

Kolmogorov, A. N. 1941c On the degeneration of isotropic turbulence in an incompressible viscous fluid. Dokl. Akad. Nauk SSSR 31, 538-541.

Kolmogorov, A. N. 1962 A refinement of previous hypotheses concerning the local structure of turbulence in a viscous incompressible fluid at high reynolds number. J. Fluid Mech. 13, 82-85.

Krogstad, P.-A. \& DAVIDSOn, P. A. 2010 Is grid turbulence saffman turbulence? J. Fluid Mech. 642, 373-394.

Krogstad, P.-A. \& Davidson, P. A. 2011 Freely decaying, homogeneous turbulence generated by multi-scale grids. J. Fluid Mech. 680, 417-434.

Krummholz, M. R. \& McKeE, C. F. 2005 A general theory of turbulenceregulated star formation, from spirals to ultraluminous infrared galaxies. Astrophys. J. 630, 250-268. 
Kuchemann, D. 1965 The aerodynamic design of aircraft. Progress in aeronautical sciences 6, 271.

KuRIAN, T. \& Fransson, J. H. M. 2009 Grid-generated turbulence revisited. Fluid Dyn. Res. 41, 021403.

Landau, L. D. \& Lifschitz, E. M. 1959 Fluid Mechanics. Pergamon Press, translated from Russian by J. B. Sykes and W. H. Reid.

Lavoie, P., DJenidi, L. \& Antonia, R. A. 2007 Effects of initial conditions in decaying turbulence generated by passive grids. J. Fluid Mech. 585, 395-420.

Laws, E. M. \& LiVesey, J. L. 1978 Flow through screens. Annu. Rev. Fluid Mech. 10, 247-266.

Lin, C. C. 1948 Note on the law of decay of isotropic turbulence. Proc. Natl. Acad. Sci. U.S.A. 34, 540-543.

LiN, Y. K. \& ARIARATNAM, S. T. 1980 Stability of bridge motion in turbulent winds. J. Struct. Mech. 8, 1-15.

Ling, S. C. \& HuAng, T. T. 1970 Decay of weak turbulence. Phys. Fluids 13, $2912-2924$.

LOITSYANSKII, L. G. 1939 Some basic regularities of an isotropic turbulent flow. Trudy TsAGI 440, 3-23.

DI LORENZO, F. 2014 Scale-dependent response of fluid turbulence under variation of the large-scale forcing. PhD thesis, Georg-August-Universität Göttingen. 
MAKITA, H. 1991 Realization of a large-scale turbulence field in a small wind tunnel. Fluid Dyn. Res. 8, 53-64.

Maurer, J., TABeling, P. \& Zocchi, G. 1994 Statistics of turbulence between two counterrotating disks in low-temperature helium gas. Europhys. Lett. 26, $31-36$.

McGhee, R. J., Beasley, W. D. \& Foster, J. M. 1984 Recent modifications and calibration of the langley low-turbulence pressure tunnel. Tech. Rep. 2328. NASA.

Meldi, M., Sagaut, P. \& LuCor, D. 2011 A stochastic view of isotropic turbulence decay. J. Fluid Mech. 668, 351-362.

Meneveau, C. \& Sreenivasan, K. R. 1987a The multifractal spectrum of the dissipation field in turbulent flows. Nucl. Phys. B 2, 49-76.

Meneveau, C. \& SReenivasan, K. R. $1987 b$ Simple multifractal cascade model for fully developed turbulence. Phys. Rev. Lett. 59, 1424-1427.

Millikan, C. B., Smith, J. E. \& Bell, R. W. 1948 High-speed testing in the southern california cooperative wind tunnel. J. Aeronaut. Sci. 15, 69-88.

Mohamed, M. S. \& LARUe, J. C. 1990 The decay power law in grid-generated turbulence. J. Fluid Mech. 219, 195-214.

Monin, A. S. \& Yaglom, A. M. 2007 Statistical Fluid Mechanics, , vol. Volume II. Dover Publications, Inc. 
Mortimer, C. E. 2001 Chemie. Das Basiswissen der Chemie. Georg Thieme Verlag.

Munk, M. 1921 On a new type of wind tunnel. Tech. Rep. 60. National Advisory Comittee for Aeronautics.

MunK, M. M. \& MilleR, E. W. 1926 The variable density wind tunnel of the national advisory committee for aeronautics. Tech. Rep. 227. Langley Memorial Aeronautical Laboratory.

MYDLARSKI, L. \& WARHAFT, Z. 1996 On the onset of high-reynolds-number grid-generated wind tunnel turbulence. J. Fluid Mech. 320, 331-368.

NAVIER, C. L. M. 1827 Sur les lois du mouvement des fluids. Comptes Rendus des Seances de l'Academie des Sciences 6, 389-440.

OswatitsCH, K. \& WIEGHARDT, K. 1987 Ludwig prandtl and his kaiser-wilhelminstitut. Annu. Rev. Fluid Mech. 19, 1-26.

PANKhURST, R. C. 1972 Aerodynamics at npl, 1917-1970. Nature 238, 375-380.

Peinke, J., Friedrich, R., Chillá, F., Chabaud, B. \& Naert, A. 1996 Statistical dependency of eddies of different sizes in turbulence. Z. Phys. B 101, $157-159$

PEROT, J. B. 2011 Determination of the decay exponent in mechanically stirred isotropic turbulence. AIP Advances 1, 022104. 
Perot, J. B. \& DE BRUyn Kops, S. M. 2006 Modeling turbulent dissipation at low and moderate reynolds numbers. J. Turbulence 7, 1-14.

Poorte, R. E. G. \& A., B. 2002 Experiments on the motion of gas bubbles in turbulence generated by an active grid. J. Fluid Mech. 461, 127-154.

Pope, S. B. 2000 Turbulent Flows. Cambridge University Press.

PRoudman, I. \& REID, W. H. 1954 On the decay of a normally distributed and homogeneous turbulent velocity field. Phil. Trans. Roy. Soc. Lond. A 247, $163-189$.

Renner, C., Peinke, J. \& Friedrich, R. 2001 Experimental indications for markov properties of small-scale turbulence. J. Fluid Mech. 433, 383-409.

REYNOLDS, O. 1883 An experimental investigation of the circumstances which determine whether the motion of water shall be direct or sinuous, and of the law of resistance in parallel channels. Phil. Trans. Roy. Soc. Lond. A 174, 935-982.

RICHARDSON, L. F. 1922 Weather prediction by numerical process. Cambridge University Press.

SAFFMAN, P. G. $1967 a$ The large-scale structure of homogeneous turbulence. $J$. Fluid Mech. 27, 581-593.

SAfFmAn, P. G. $1967 b$ Note on decay of homogeneous turbulence. Phys. Fluids 10, 1349 . 
SCHEWE, G. 1983 On the force fluctuations acting on a circular cylinder in crossflow from subcritical up to transcritical reynolds numbers. J. Fluid Mech. 133, 265285.

SCHEWE, G. 2013 Reynolds-number-effects in flow around a rectangular cylinder with aspect ratio 1:5. J. Fluid Struct. 39, 15-26.

SCHLiChting, H. 1956 The variable density high speed cascade wind tunnel of the deutsche forschungsanstalt für luftfahrt braunschweig. Tech. Rep. 91. Advisory Group for Aeronautical Research and Development.

Schubauer, G. B. \& Spangenberg, W. G. 1948 Effect of screens in wide-angle diffusers. Tech. Rep.. NACA.

Seber, G. A. F. \& Wild, C. J. 2003 Nonlinear Regression. NJ: WileyInterscience.

SHE, Z.-S. \& LÉVÊQUE, E. 1994 Universal scaling laws in fully developed turbulence. Phys. Rev. Lett. 72, 336-339.

SHEN, X. \& WARHAFT, Z. 2002 Longitudinal and transverse structure functions in sheared and unsheared wind-tunnel turbulence. Phys. Fluids 14, 370-381.

Siebert, H., Lehmann, K. \& Wendisch, M. 2006 Observations of small-scale turbulence and energy dissipation rates in the cloudy boundary layer. J. Atmos. Sci. 65, 1451-1466. 
Simmons, L. F. G. \& SAlTER, C. 1934 Experimental investigation and analysis of the velocity variations in turbulent flow. Proc. Roy. Soc. Lond. A 145, 212-234.

Singh, A. K. \& Bhadauria, B. S. 2009 Finite difference formulae for unequal sub-intervals using lagrange's interpolation formula. Int. Journal of Math. Analysis 3, 815-827.

Sinhuber, M., Bodenschatz, E. \& Bewley, G. P. 2015 Decay of turbulence at high reynolds numbers. Phys. Rev. Lett. 114, 034501.

SIRIVAT, A. \& WARHAFT, Z. 1983 The effect of a passive cross-stream temperature gradient on the evolution of temperature variance and heat flux in grid turbulence. J. Fluid Mech. 128, 323-346.

SKRBEK, L. \& StALP, S. R. 2000 On the decay of homogeneous isotropic turbulence. Phys. Fluids 12, 1997-2019.

Smits, A. J. \& Hultmark, M. 2014 Nanoscale instrumentation for measuring turbulence. In 19th Australasian Fluid Mechanics Conference.

Speziale, C. G. \& Bernard, P. S. 1992 The energy decay in self-preserving isotropic turbulence revisited. J. Fluid Mech. 241, 645-667.

SREENIVASAN, K. R. 1998 An update on the energy dissipation rate in isotropic turbulence. Phys. Fluids 10, 528-529.

SReEnivasan, K. R. \& Dhruva, B. 1998 Is there scaling in high-reynoldsnumber turbulence? Progr. Theor. Phys. Supp. 130, 103-120. 
Sreenivasan, K. R., Tavoularis, S., Henry, R. \& Corrsin, S. 1980 Temperature fluctuations and scales in grid-generated turbulence. J. Fluid Mech. 100, 597-621.

Stalp, S. R., SkRbeK, L. \& Donnelly, R. J. 1999 Decay of grid turbulence in a finite channel. Phys. Rev. Lett. 82, 4831-4834.

StOKES, G. G. 1845 On the theories of the internal friction of fluids in motion, and of the equilibrium and motion of elastic solids. Tran. Camb. Phil. Soc. 8, $287-305$.

Stolovitzky, G. \& SReEnivasan, K. R. 1993 Scaling of structure functions. Phys. Rev. E 48, R33-R36.

TAYLOR, G. I. 1935 Statistical theory of turbulence: Parts i-iii. Proc. Lond. Math. Soc. 20, 196-212.

TAYlor, G. I. 1938 The spectrum of turbulence. Proc. Roy. Soc. Lond. A 164, $476-490$.

Thormann, A. \& Meneveau, C. 2014 Decay of homogeneous, nearly isotropic turbulence behind active fractal grids. Phys. Fluids 3+, 025112.

Toschi, F., Amati, G., Succi, S., Benzi, R. \& Piva, R. 1999 Intermittency and structure functions in channel flow turbulence. Phys. Rev. Lett. 82, 5044-5047.

Tropea, C., Yarin, A. \& Foss, J. F., ed. 2007 Springer Handbook of Experimental Fluid Mechanics. Springer-Verlag. 
Tsinober, A., Kit, E. \& Dracos, T. 1992 Experimental investigation of the field of velocity gradients in turbulent flows. J. Fluid Mech. 242, 169-192.

Uberoi, M. S. 1963 Energy transfer in isotropic turbulence. Phys. Fluids 6, 10481056.

Uberoi, M. S. \& WALlis, S. 1966 Small axisymmetric contraction of grid turbulence. J. Fluid Mech. 24, 539-543.

Valente, P. C. \& VAssilicos, J. C. 2011 he decay of turbulence generated by a class of multiscale grids. J. Fluid Mech. 687, 300-340.

Vallikivi, M., Hultmark, M., Bailey, S. \& Smits, A. 2011 Turbulence measurements in pipe flow using a nano-scale thermal anemometry probe. Exp. Fluids 51, 1521-1527.

Van Atta, C. W. \& Antonia, R. A. 1980 Reynolds number dependence of skewness and flatness factors of turbulent velocity derivatives. Phys. Fluids $\mathbf{2 3}$, $252-257$.

VAn AtTA, C. W. \& Chen, W. Y. 1968 Correlation measurements in grid turbulence using digital harmonic analysis. J. Fluid Mech. 34, 497-515.

Vincent, A. \& Meneguzzi, M. 1991 The spatial structure and statistical properties of homogeneous turbulence. J. Fluid Mech. 225, 1-20. 
WANG, X., Feng, X., YAnG, C. \& MaO, Z.-S. 2014 Energy dissipation rates of newtonian and non-newtonian fluids in a stirred vessel. Chem. Eng. Technol. 37, $1575-1582$.

WARHAFt, Z. 2000 Passive scalars in turbulent flows. Annu. Rev. Fluid Mech. 32, $203-240$.

WARHAFT, Z. \& LuMLEY, J. L. 1978 An experimental study of the decay of temperature fluctuations in grid-generated turbulence. J. Fluid Mech. 88, 659684.

VAN DE WATER, W. \& Herweijer, J. A. 1999 High-order structure functions of turbulence. J. Fluid Mech. 387, 3-37.

White, C. M., Karpetis, A. N. \& Sreenivasan, K. R. 2002 High-reynoldsnumber turbulence in small apparatus: grid turbulence in cryogenic liquids. $J$. Fluid Mech. 452, 189-197.

Wollaston, W. H. 1813 A method of drawing extremely fine wires. Phil. Trans. Roy. Soc. Lond. 103, 114-118.

WYATT, L. A. 1955 Energy and spectra in decaying homogeneous turbulence. $\mathrm{PhD}$ thesis, University of Manchester.

YOON, K. \& WARHAFT, Z. 1990 The evolution of grid-generated turbulence under conditions of stable thermal stratification. J. Fluid Mech. 215, 601-638. 
Zagarola, M. V. \& SMits, A. J. 1997 Sacling of the mean velocity profile for turbulent pipe flow. Phys. Rev. Lett. 78, 239-242.

Zhou, T. \& Antonia, R. A. 2000 Reynolds number dependence of the smallscale structure of grid turbulence. J. Fluid Mech. 406, 81-107.

Zimmermann, R., Xu, H., Gasteuil, Y., Bourgoin, M., Volk, R., PinTOn, J.-F., Bodenschatz, E. \& FOR Turbulence Research, I. C. 2010 The lagrangian exploration module: An apparatus for the study of statistically homogeneous and isotropic turbulence. Rev. Sci. Instrum. 81, 055112. 


\section{Curriculum Vitae}

\section{Personal Information}

Name Michael Sinhuber

Date of birth July 7, 1985

Nationality German

\section{Academic Background}

06/2011-dato Doctoral studies in Physics

Max Planck Institute for Dynamics and Self-Organization, Göttingen

Georg-August-Universität, Göttingen

International Max Planck Research School for Physics of Biological and Complex Systems (IMPRS PBCS)

04/2006-02/2011 Diploma studies in Physics

Westfälische Wilhelms-Universität, Münster

07/2005 Abitur

Neues Gymnasium, Oldenburg 


\section{Published Publications}

M. Sinhuber, E. Bodenschatz and G. P. Bewley 2015

Decay of Turbulence at High Reynolds Numbers

Phys. Rev. Let. 114, 034501.

E. Bodenschatz, G. P. Bewley, H. Nobach, M. Sinhuber and

H. Xu. 2014 Variable density turbulence tunnel facility.

Rev. Sci. Instrum. 85, 093908.

\section{Awards and Grants}

2007-2011 Stipend of the 'Studienstiftung des Deutschen Volkes'

\section{Teaching Certificate}

10/2013-dato Göttinger Zertifikatsprogramm

Division of Teaching and Learning in Higher Education

Georg-August-Universität, Göttingen

\section{Teaching Experience}

10/2012-03/2015 Lecturer, Georg-August-Universität, Göttingen

$\diamond$ Seminar "Current questions in turbulence research"

08/2011-10/2012 Teaching assistant, Georg-August-Universität, Göttingen

$\diamond$ Thermodynamics and statistical mechanics

$\diamond$ Quantum mechanics 
10/2008-07/2010 Teaching assistant, Westfälische Wilhelms-Universität Münster

$\diamond$ Thermodynamics and Electrodynamics

$\diamond$ Mechanics

$\diamond$ Analysis

$\diamond$ Linear Algebra

\section{Selected Talks and Conferences}

11/2014 MPIDS-PKU Fluid Dynamics Symposium

Decay of Turbulence at High Reynolds Numbers

03/2014 New Challenges in Turbulence Research III

Reynolds Number Dependencies In Grid Turbulence

09/2013 European Turbulence Conference 14

Reynolds Number Dependencies In Classical Grid Turbulence

11/2012 Instituts-Retreat Burg Ludwigstein

The "other" turbulence problem

07/2012 Workshop Selbstorganisation und Komplexität 2012

Grid Turbulence: Experiments at the Göttingen Turbulence

Tunnel

05/2010 Self-Organization in Turbulent Plasmas and Fluids

Direct numerical simulation of Rayleigh-Bénard-Equations using volume penalization

Göttingen, April 27th 2015 
Curriculum Vitae 


\section{Acknowledgments}

I would like to thank Eberhard Bodenschatz for giving me the great opportunity to conduct my studies at the Max-Planck-Institute for Dynamics of Self-Organization, supervising my thesis, for fruitful discussions, for wise advice, for helpful support and the for the trust that a trained theoretician can conduct experimental studies in one of the most advanced wind tunnels in the world.

Greg Bewley spent countless hours teaching me how to work and think as an experimentalist and even more time in scientific disussions, solving problems and stimulating right ideas at the right time. Thank you!

I would like to thank Andreas Dillmann and Marcus Müller for participating in my thesis committee. Their helpful questions and advice greatly helped to improve this thesis. The studies I conducted would not have been possible without the NSTAPs developed at Princeton University and kindly provided to us by Lex Smits, Marcus Hultmark, Margit Vallikivi and Yuyang Fan. They helped set the probes probes into operation in the VDTT and never hesitated to solve any occurring problem. I like to thank Mickael Bourgoin and the organizing team for giving me the opportunity to participate in the once-in-a-lifetime experiment in the S1MA. 
Acknowledgments

None of the experiments would have been possible without the amazing technical support by Andreas Kopp, Andreas Renner, Marcel Meyer, Artur Kubitzek and Gerhard Nolte as well as Udo Schminke and his team, including especially Nils Winkelmann. I am grateful to Florian Köhler and Laura Hillmann for help in the data acquisition process. I would like to thank Antje Erdmann, Michaela Böttcher, Frauke Bergmann and Tina Trost at the PBCS office as well as Angela Meister and Sabrina Volkmar for solving all administrative and organizational problems.

I thank the whole LFPB group for having a great time, discussions and a friendly, productive environment. I especially want to thank Jennifer Jucha, Garrett Good, Michael Wilczek, Sune Wissing and Greg for proof-reading drafts of my thesis.

My parents Ute and Achim, my sister Marlene, as well as Frederik and Sune, along with the MNG and DSA crews, played an important role in keeping my sanity during my graduate studies. Thank you for that.

If it would not have been for Rudolf Friedrich, I would not have considered to conduct research on turbulence and would not have been doing this thesis. I wish I could still thank him in person. 